\title{
Search for New Physics in the Missing Transverse Energy + Dijet Channel at CDF
}

by

Daniel Montgomery MacQueen

A thesis submitted in conformity with the requirements

for the degree of Doctor of Philosophy

Graduate Department of Physics

University of Toronto

Copyright (C) 2009 by Daniel Montgomery MacQueen 


\begin{abstract}
Search for New Physics in the Missing Transverse Energy + Dijet Channel at CDF

\author{
Daniel Montgomery MacQueen \\ Doctor of Philosophy \\ Graduate Department of Physics \\ University of Toronto
}

2009

This thesis presents the results of a signature-based search for new physics using the exclusive dijet plus missing transverse energy data sample from $2 \mathrm{fb}^{-1}$ of $p \bar{p}$ collisions at $\sqrt{s}=1.96 \mathrm{TeV}$ collected with the Collider Detector at Fermilab (CDF). A study is made of the production of events with two high energy jets and large missing transverse energy (missing $E_{T}$, or $E_{T}$ ) in a kinematic regime requiring the scalar sum of the $E_{T}$ of the two jets (referred to here as $H_{T}$ ) to be greater than $125 \mathrm{GeV}$ and the event $E_{T}$ to be above $80 \mathrm{GeV}$. A second kinematic region is also examined, with the $\not_{T}$ cut increased to $100 \mathrm{GeV}$ and the $H_{T}$ cut increased to $225 \mathrm{GeV}$. The number of events observed in the data is within 0.43 standard deviations of the expected number of background events in the low kinematic region, and within 0.34 standard deviations in the high kinematic region. Based on these results, 95\% C.L. lower mass limits for scalar leptoquarks are extracted: $190 \mathrm{GeV} / c^{2}$ for 1 st generation, $190 \mathrm{GeV} / c^{2}$ for 2 nd generation, and $178 \mathrm{GeV} / c^{2}$ for $3 \mathrm{rd}$ generation production. The results are also interpreted in terms of cross-section limits on generic minimal supersymmetric (MSSM) models.
\end{abstract}




\section{Acknowledgements}

No Ph.D. thesis, especially in a field like experimental high-energy physics where papers are routinely published with hundreds of credited authors, is truly completed by one person's sole efforts. First, I must thank Bob Orr, my supervisor, for his advice while carrying out this analysis and writing this thesis. I would also like to thank Amanda Peet, John Martin, and Peter Krieger for their support and advice during their time on my Ph.D. committee, and my external examiner Scott Menary for his input.

This analysis could not have been carried out without the contributions of Hugo Beauchemin, Pierre Savard, Eric James, Kevin Burkett, and Pier-Olivier Deviveiros. Hugo deserves special thanks for staying closely involved with this analysis even after changing experiments, institutions, and continents.

I would like to thank the many other physicists who have worked on the CDF experiment during my time as a doctoral student, as well as the University of Toronto and Fermilab support staff. Special thanks must go to Song Ming Wang for his encouragement and advice; Charles Plager for his computing help; and the conveners of the physics groups who guided this analysis through the blessing process - Aron Soha and Conor Henderson from the Very Exotic Phenomena Working Group, Dave Toback and Monica D'Onofrio from the Supersymmetry Working Group, and Chris Hays and Ben Brau from the Exotics Working Group. I also thank Mario Martinez-Perez, Kaori Maeshima, and Mousumi Datta, the members of the godparenting committee who are helping to shepherd this analysis toward eventual publication. I would like to both thank and apologize to Simona Rolli, whose ideas for extending the leptoquark interpretation we were unable to get to work.

I would like to thank the other faculty members of the IPP Canada CDF group with whom I have worked: Pekka Sinervo, William Trischuk, and Andreas Warburton. The graduate students and post-docs who worked alongside me in Toronto and at Fermilab must also be thanked, especially Jean-Francois Arguin, Andrew Hamilton, Kostas Ko- 
rdas, Stan Lai, Kalen Martens, Rachid Mazini, Michael Riveline, Shabnaz Pashapour, Simon Sabik, Rob Snihur, Teresa Spreitzer, Bernd Steltzer, Oliver Steltzer-Chilton, and Ian Vollrath.

I would also like to reach further back in my time as a student researcher and thank my former supervisor Doug Gingrich and the rest of the ATLAS group at the University of Alberta, as well as Dave Hutcheon and the other TRIUMF physicists I worked with during my summer on Experiment 704.

On a more personal note, I would like to thank my friends for their support during this process. Among them must be mentioned the semi-infamous "men of 510": Nick Rudzik, Bart Wilson, Matt Doyle, Mounir Bashour, Daniel Saturnino, Sasha Bataglin, Xunming Huang, Peter Tang, and Adnan Hussain. My friends back in Edmonton have also helped keep me sane, especially Stanley Woo, Kyle Jago, Lee \& Mary Conrad, and Tammy Valgardson.

I would also like to thank my family for their support: Grandma Skakum; Gram and Ross Hoggart; my parents, Craig and Sharon; Dennis Skakum and my many other aunts, uncles, and cousins; my brother Sandy and my sister-in-law Emily; and their children Ethan and Erika (who, despite being babies living in another province, managed to help support me during the final year of my analysis. They never failed to cheer me up whenever new pictures arrived.)

Finally, all the aforementioned colleagues who have become my friends over the years must be thanked a second time. I wish them all the best of luck in their future research as many of them begin their work at the LHC. 


\section{Contents}

1 Preface 1

1.1 The Standard Model . . . . . . . . . . . . . . . . 1

1.2 Beyond the Standard Model . . . . . . . . . . . . . . . 4

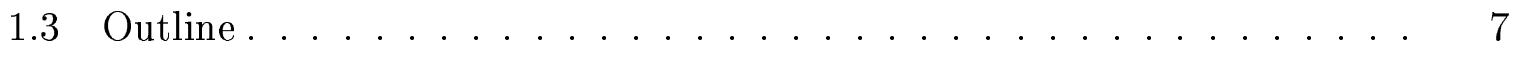

2 The Tevatron and the Collider Detector at Fermilab $\quad 8$

2.1 The Tevatron Accelerator Chain . . . . . . . . . . . . . 9

2.1.1 The Proton Source ..................... 9

2.1 .2 The Main Injector . . . . . . . . . . . . . 10

2.1.3 The Antiproton Source ................. 11

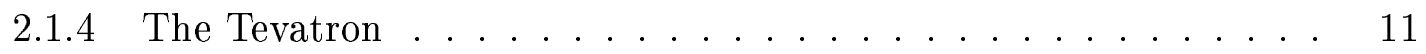

2.2 The Collider Detector at Fermilab . . . . . . . . . . . . 12

2.2.1 Co-ordinate System and Related Quantities . . . . . . . . 13

2.2.2 The Silicon Detectors . . . . . . . . . . . . 14

2.2.3 The Central Outer Tracker . . . . . . . . . . . 16

2.2 .4 The Calorimeters .................... 17

2.2.5 The Muon Detectors ................. 19

2.2.6 The Cherenkov Luminosity Counters . . . . . . . . . . 20

2.3 The Triggers and Data Acquisition Systems . . . . . . . . . . . 20 
3 Data Samples $\quad 23$

3.1 Run Lists and Luminosities . . . . . . . . . . . . . 23

3.2 The High- $p_{T}$ Lepton Samples . . . . . . . . . . . . . 25

3.2.1 The High- $p_{T}$ Muon Sample . . . . . . . . . . . . . 25

3.2 .2 The High- $p_{T}$ Electron Sample ............... 26

3.3 The MET45 Data Sample ................... 27

3.3.1 MET45 Trigger Efficiency _............... 27

4 Selection Cuts $\quad 31$

4.1 Isolated Track Veto . . . . . . . . . . . . . . . . . . 31

4.2 Jet Definition and Cuts . . . . . . . . . . . . 32

4.2.1 Jet Energy Scale and Corrections . . . . . . . . . . . 33

4.2 .2 Dijet Selection . . . . . . . . . . . . . 35

4.3 Clean-up Cuts . . . . . . . . . . . . . . . . 38

4.4 Kinematic Regions . . . . . . . . . . . . . . . . 39

5 Standard Model Backgrounds $\quad 40$

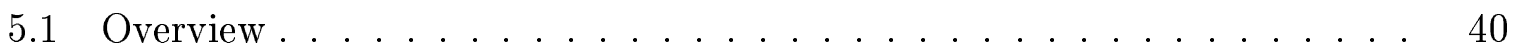

5.2 Electroweak Backgrounds ..................... 41

5.2.1 $Z$ and $W$ Identification in High- $p_{T}$ Muon Samples . . . . . . . 41

5.2.2 $Z$ and $W$ Identification in High- $p_{T}$ Electron Samples . . . . . . 44

$5.2 .3 \quad Z$ and $W$ Inclusive Cross-sections . . . . . . . . . . . 47

5.2.4 $Z$ and $W+$ Dijet Cross-sections ............ 50

5.2.5 Z $\rightarrow \nu \bar{\nu}+$ Dijets Background ............ 56

5.2.6 $W^{-} \rightarrow l \bar{\nu}_{l}+$ Dijets Background............ 61

5.2.7 $Z \rightarrow l \bar{l}+$ Dijets Background ............. 62

5.2.8 Cross-checks with ALPGEN Monte Carlo . . . . . . . . . 62

5.3 QCD Backgrounds ........................... 64 
5.4 Other Backgrounds . . . . . . . . . . . . . . . . . 72

5.4 .1 Top Backgrounds . . . . . . . . . . . . . . . 72

5.4 .2 Photon + Jets Backgrounds . . . . . . . . . . . . 74

5.4 .3 Non-Collision Backgrounds . . . . . . . . . . . . . 76

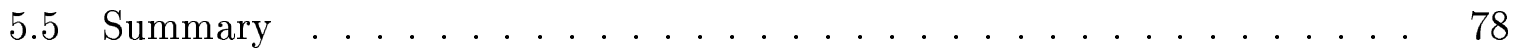

6 Leptoquarks $\quad 91$

6.1 Leptoquark Models and Types . . . . . . . . . . . . . . . . . . . 91

6.2 Signal Monte Carlo . . . . . . . . . . . . . . . . . . . . . . . 95

6.3 Previous Leptoquark Searches _. . . . . . . . . . . . . . 96

$\begin{array}{lll}7 & \text { Supersymmetry } & 99\end{array}$

7.1 The Minimal Supersymmetric Extension to the Standard Model . . . . 99

7.1.1 The Hierarchy Problem and Supermultiplets . . . . . . . . . . 99

$7.1 .2 \quad$ R-parity . . . . . . . . . . . . . . . . . . . . 101

7.1 .3 Mass Eigenstates . . . . . . . . . . . . . . 103

7.1.4 Supersymmetry Models . . . . . . . . . . . . . . . . . . . 104

7.1.5 Squark and gluino production . . . . . . . . . . 107

7.1.6 Squark and gluino decay $\ldots \ldots \ldots \ldots . \ldots \ldots$

7.2 Signal Monte Carlo . . . . . . . . . . . . . . . . . . . . . . . 109

7.3 Previous squark/gluino searches at the Tevatron . . . . . . . . . . . 111

8 Analysis and Results 113

8.1 Results from Data and Limit Calculations . . . . . . . . . . . 113

8.2 Uncertainties on Signal Acceptance . . . . . . . . . . . . . 116

8.2.1 Choice of Parton Distribution Function . . . . . . . . . 116

8.2 .2 Jet Energy Scale . . . . . . . . . . . . . . . . . 118

8.2.3 Initial and Final State Radiation $\ldots \ldots \ldots \ldots \ldots$

8.2 .4 Luminosity . . . . . . . . . . . . . . . . . . 119 
8.2.5 Monte Carlo Statistics . . . . . . . . . . . . . 119

8.2.6 Efficiency of Clean-up Cuts . . . . . . . . . . 120

8.3 Leptoquark Interpretation . . . . . . . . . . . . . . 121

8.3.1 Expected Signal Yields . . . . . . . . . . . . . . 122

8.3.2 Uncertainties and Event Limits . . . . . . . . . . . 122

8.3.3 Leptoquark Generations . . . . . . . . . . . . 123

8.3.4 Cross-section and Mass Limits . . . . . . . . . . . . . 124

8.3.5 Kinematic Comparisons ............... 124

8.4 Supersymmetric Interpretation . . . . . . . . . . . . . . 133

8.4.1 Expected Signal Yields . . . . . . . . . . . . . 134

8.4.2 Uncertainties and Event Limits . . . . . . . . . . . 139

8.4.3 Cross-section Limits . . . . . . . . . . . . . . . 142

8.4.4 Kinematic Comparisons ................ 143

9 Conclusion $\quad 146$

9.1 Summary of Results . . . . . . . . . . . . . . . 146

9.2 Discussion . . . . . . . . . . . . . . . . 147

9.3 Possibilities for Future Research . . . . . . . . . . . . . . . . 148

$\begin{array}{lr}\text { Bibliography } & 150\end{array}$ 


\section{List of Tables}

1.1 Particle content of the Standard Model . . . . . . . . . . . . 3

5.1 Input parameters and results for the $W$ inclusive cross section measurements 48

5.2 Input parameters and results for the $Z$ inclusive cross section measurements 49

5.3 Input parameters and results for the $W$ plus dijet cross section measure-

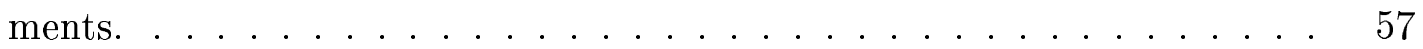

5.4 Input parameters and results for the $Z$ plus dijet cross section measurements. 58

5.5 Combined $W / Z+$ jet cross section measurements used to estimate backgrounds in the dijet plus missing $E_{T} \ldots \ldots \ldots . \ldots 59$

5.6 Values for $R$ obtained from MCFM theoretical calculations. . . . . . . 60

5.7 Predicted numbers of $Z \rightarrow \nu \bar{\nu}$ events. . . . . . . . . . . . 61

5.8 Internal consistency check of the QCD background calculation. . . . . 75

5.9 Single-top and $t \bar{t}$ background contribution. . . . . . . . . 75

5.10 Summary of estimated SM background contributions to the dijet plus $\not_{T}$ candidate samples along with the number of observed events in data. . . 79

6.1 Table of possible first-generation scalar (spin-0) leptoquark types. . . . . 93

6.2 Table of possible first-generation vector (spin-1) leptoquark types. . . . . 94

6.3 Calculated NLO cross sections for different values of the leptoquark mass 97

7.1 Chiral and gauge supermultiplets of the MSSM. . . . . . . . . . . 102

7.2 Mass and gauge eigenstates of the MSSM. . . . . . . . . . 105 
7.3 MSSM particle masses examined in this analysis. . . . . . . . . . 110

8.1 95\% CL upper limits on the number of non-SM signal events contributing to the low kinematic region candidate sample $\ldots \ldots \ldots \ldots . \ldots 114$

$8.295 \%$ CL upper limits on the number of non-SM signal events contributing to the high kinematic region candidate sample . . . . . . . . 115

8.3 $A_{s i g}$ from Monte Carlo as a function of second-generation leptoquark mass. 127

8.4 Expected number of signal events as a function of second-generation leptoquark mass. . . . . . . . . . . . . . . 128

8.5 Scale factors and associated uncertainties in the low kinematic region for leptoquarks. . . . . . . . . . . . . . . . . . 129

8.6 Scale factors and associated uncertainties in the high kinematic region for leptoquarks. . . . . . . . . . . . . . . . . . 129

$8.7 \Delta A_{s i g}$ for $150 \mathrm{GeV} / c^{2}$ leptoquarks $\ldots \ldots \ldots \ldots \ldots \ldots$

$8.8 \Delta A_{s i g}$ for $180 \mathrm{GeV} / c^{2}$ leptoquarks $\ldots \ldots \ldots \ldots \ldots \ldots$

8.9 95\% CL lower limits on scalar leptoquark mass and production cross-sections. 131

8.10 PYthiA cross-sections and $A_{s i g}$ from Monte Carlo for the MSSM spectra examined. . . . . . . . . . . . . . . . 133

8.11 Leading order yields from PYTHIA for SUSY mass spectra examined. . . . 134

8.12 Scale factors and associated uncertainties in the low kinematic region for MSSM spectrum S1. . . . . . . . . . . . . . 135

8.13 Scale factors and associated uncertainties in the high kinematic region for

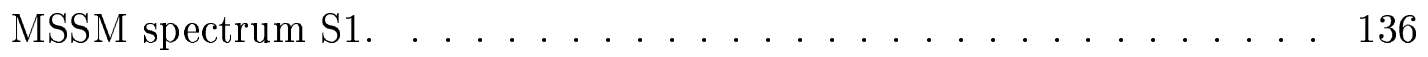

8.14 Scale factors and associated uncertainties in the low kinematic region for

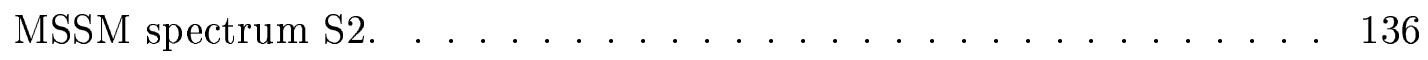

8.15 Scale factors and associated uncertainties in the high kinematic region for MSSM spectrum S2. . . . . . . . . . . . . . . 137 
8.16 Scale factors and associated uncertainties in the low kinematic region for MSSM spectrum S3. ....................... 137

8.17 Scale factors and associated uncertainties in the high kinematic region for MSSM spectrum S3. . . . . . . . . . . . . . 138

8.18 Scale factors and associated uncertainties in the low kinematic region for MSSM spectrum S4. . . . . . . . . . . . . 138

8.19 Scale factors and associated uncertainties in the high kinematic region for MSSM spectrum S4. . . . . . . . . . . . . . 139

$8.20 \Delta A_{\text {sig }}$ for MSSM spectrum S1 . . . . . . . . . . . . . 140

$8.21 \Delta A_{s i g}$ for MSSM spectrum S2 . . . . . . . . . . . 140

$8.22 \Delta A_{\text {sig }}$ for MSSM spectrum S3 . . . . . . . . . . . . 141

$8.23 \Delta A_{s i g}$ for MSSM spectrum $\mathrm{S} 4 \ldots \ldots \ldots \ldots$

8.24 95\% CL upper limits on the number of MSSM signal events contributing to the candidate samples for the four spectra examined . . . . . . . . 142

8.25 Cross-section 95\% CL a priori and observed upper limits for all four SUSY spectra, compared to PYTHIA leading order cross-sections. . . . . . . . 143 


\section{List of Figures}

1.1 Typical Feynman diagram producing a dijet $+\not_{T}$ signature. . . . . . . 6

2.1 The Fermilab Tevatron accelerator chain. . . . . . . . . . . . . . 9

2.2 Cross-sectional view of half of the Collider Detector at Fermilab. . . . . . 13

2.3 Longitudinal view of the CDF inner detector. . . . . . . . . . 15

2.4 Block diagram of the data flow at CDF. . . . . . . . . . . 21

3.1 Bin-per-bin efficiency distribution of the MET45 trigger as a function of the reconstructed $E_{T} \ldots \ldots \ldots \ldots \ldots$

3.2 Cumulative efficiency distribution for the MET45 trigger as a function of the reconstructed $E_{T} \ldots \ldots \ldots \ldots$

5.1 Comparison of electron kinematic distributions between ALPGEN Monte Carlo and data for $W^{-} \rightarrow e \bar{\nu}_{e}+$ jets candidate sample. . . . . . . .

5.2 Comparison of electron kinematic distributions between ALPGEN Monte Carlo and data for $Z \rightarrow e \bar{e}+$ jets candidate sample. . . . . . . . . 64

5.3 Comparison of muon kinematic distributions between ALPGEN Monte Carlo and data for $W^{-} \rightarrow \mu \bar{\nu}_{\mu}+$ jets candidate sample. . . . . . . .

5.4 Comparison of muon kinematic distributions between ALPGEN Monte Carlo and data for $Z \rightarrow \mu \bar{\mu}+$ jets candidate sample. . . . . . . . . . 66

5.5 Comparison of jet kinematic distributions between ALPGEN Monte Carlo and data for $W^{-} \rightarrow \mu \bar{\nu}_{\mu}+$ jets candidate sample. . . . . . . . . 
5.6 Comparison of jet kinematic distributions (I) between ALPGEN Monte Carlo and data for $Z \rightarrow \mu \bar{\mu}+$ jets candidate sample. . . . . . . . 68

5.7 Comparison of jet kinematic distributions (II) between ALPGEN Monte Carlo and data for $Z \rightarrow \mu \bar{\mu}+$ jets candidate sample. . . . . . . 69

5.8 Comparisons of the $Z$ invariant mass and $W$ transverse mass distributions between ALPGen Monte Carlo and data in $\mu$ samples. . . . . . . . . 70

$5.9 \Delta \phi$ between $\not_{T}$ and the third jet in the event for $W^{-} \rightarrow \tau \bar{\nu}_{\tau}$ events. . . 71

$5.10 \Delta \phi$ between $E_{T}$ and the third jet in the event for $W^{-} \rightarrow \mu \bar{\nu}_{\mu}$ events. . 72

5.11 Fit to the 3 rd jet $E_{T}$ distribution in the data. . . . . . . . 73

5.12 Fit to the 3 rd jet $E_{T}$ distribution in the simulation. . . . . . . . . 74

5.13 Comparison of event $E_{T}$ for the low kinematic region, showing the stacked contributions of each SM background process. . . . . . . . 80

5.14 Comparison of event $H_{T}$ for the low kinematic region, showing the stacked contributions of each SM background process. . . . . . . . . 81

5.15 Comparison of $E_{T}$ for low kinematic region data and SM prediction. . . 82

5.16 Comparison of $H_{T}$ for low kinematic region data and SM prediction. . . . 82

5.17 Comparison of leading jet $E_{T}$ for low kinematic region data and SM prediction. ......................... 83

5.18 Comparison of second jet $E_{T}$ for low kinematic region data and SM pre-

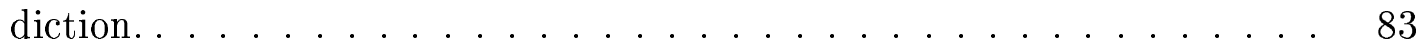

5.19 Comparison of leading jet $\eta$ for low kinematic region data and SM prediction. 84

5.20 Comparison of second jet $\eta$ for low kinematic region data and SM prediction. 84

5.21 Comparison of $\Delta \phi$ between the two jets for low kinematic region data and

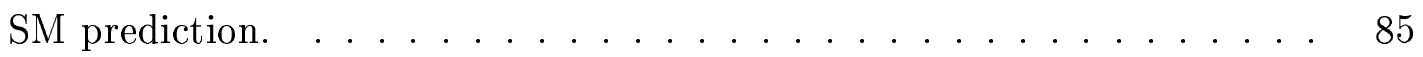

5.22 Shape comparison of $E_{T}$ for low kinematic region data and SM prediction. 85

5.23 Shape comparison of $H_{T}$ for low kinematic region data and SM prediction. 86

5.24 Comparison of $E_{T}$ for high kinematic region data and SM prediction. . . 86 
5.25 Comparison of $H_{T}$ for high kinematic region data and SM prediction. . .

5.26 Comparison of leading jet $E_{T}$ for high kinematic region data and SM

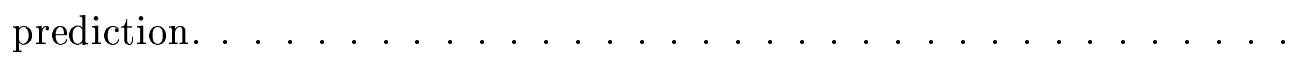

5.27 Comparison of second jet $E_{T}$ for high kinematic region data and SM pre-

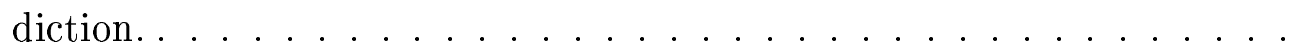

5.28 Comparison of leading jet $\eta$ for high kinematic region data and SM pre-

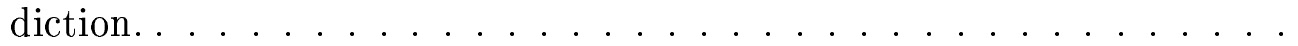

5.29 Comparison of second jet $\eta$ for high kinematic region data and SM prediction. 89

5.30 Comparison of $\Delta \phi$ between the two jets for high kinematic region data

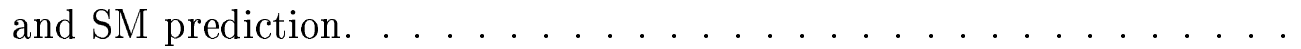

5.31 Comparison of the $H_{T}$ distribution between the SM prediction and data in the high kinematic region after removal of the jet $H_{T}$ selection cut. . . 90

7.1 Squark and gluino masses ruled out by previous searches compared to spectra examined in this analysis.

8.1 95\% cross-section limits for 1 st and 2 nd generation leptoquark pair production as a function of $M_{L Q} \ldots \ldots \ldots \ldots \ldots$

8.2 $H_{T}$ from data, background prediction, and signal prediction after $\not_{T}>$ $100 \mathrm{GeV}$ cut for $180 \mathrm{GeV} / c^{2}$ leptoquarks. . . . . . . . . . . 126

8.3 $H_{T}$ from data, background prediction, and signal prediction in high kinematic region for $180 \mathrm{GeV} / c^{2}$ leptoquarks. . . . . . . . . . . . 131

8.4 $E_{T}$ from data, background prediction, and signal prediction in high kinematic region for $180 \mathrm{GeV} / c^{2}$ leptoquarks. . . . . . . . . . . . . 132

8.5 $H_{T}$ from data, background prediction, and signal prediction after $\not_{T}>$ $100 \mathrm{GeV}$ cut for MSSM spectrum S2. . . . . . . . . . . . 144

8.6 $H_{T}$ from data, background prediction, and signal prediction in high kinematic region for MSSM spectrum S2. . . . . . . . . . . . 145 
8.7 $E_{T}$ from data, background prediction, and signal prediction in high kinematic region for MSSM spectrum S2. . . . . . . . . . . . 145 


\section{Chapter 1}

\section{Preface}

\subsection{The Standard Model}

The Standard Model [1] (abbreviated SM) is the theory currently used to explain the behaviour of matter at a subatomic level. Table 1.1 shows the particle content of the SM. In the SM, the fundamental particles of matter are divided into two types of spin$1 / 2$ fermions. The first type, the leptons, include the electron $e$, the muon $\mu$, and the tau $\tau$, and their associated neutrinos $\nu_{e}, \nu_{\mu}$, and $\nu_{\tau}$. They can also be divided into three generations, with $e$ and $\nu_{e}$ being the first generation, $\mu$ and $\nu_{\mu}$ being the second generation, and $\tau$ and $\nu_{\tau}$ being the third. The other fundamental fermions, the quarks, can be divided into the first generation up and down quarks $u$ and $d$, the second generation strange and charm quarks $s$ and $c$, and the third generation bottom and top quarks $b$ and $t$. In contexts where the flavour or generation of a fundamental fermion is irrelevant, ignored, or generalized, charged leptons are denoted with $l$, neutrinos with $\nu$ or $\nu_{l}$, and quarks with $q$. Each of these fermions has an anti-matter counterpart, represented as $\bar{l}$, $\bar{\nu}_{l}$, or $\bar{q}$, with the same spin and mass but opposite electric and colour charges.

The SM also describes three forces between the fundamental particles, which are mediated by four spin-1 gauge bosons. These forces adhere to the local gauge symmetries 
$S U(3)_{C} \times S U(2)_{W} \times U(1)_{Y}$. The $S U(3)_{C}$ symmetry represents the strong, or colour, force, mediated by the gluons $g$. Quantum chromodynamics, or QCD, is the name of the theory governing their interactions [2].

The $S U(2)_{W} \times U(1)_{Y}$ symmetry represents weak isospin and hypercharge symmetry, respectively, and represents a unification between the electromagnetic force mediated by the photons $\gamma$ and the weak force mediated by the $W^{ \pm}$and $Z$ bosons. The two forces together are referred to as the electroweak force, and the bosons mediating them are called electroweak bosons [3]. The breaking of the symmetry between the weak and electromagnetic forces comes from the Higgs mechanism [4], which keeps the $\gamma$ massless but gives the $W^{ \pm}$and $Z$ bosons mass. This requires the existence of a spin-0 Higgs boson $H$.

The electromagnetic force acts on all electrically charged particles, while the weak force acts on all quarks, all leptons, and the $W$ and $Z$ bosons. Quarks and gluons, the only particles with a property called colour charge, are the only particles which interact via the strong force. There are three types of colour charge, denoted "red", "blue", and "green". The nature of the strong force results in two important consequences for quarks: confinement and asymptotic freedom. Confinement is a consequence of the strength of the strong interaction, which increases with distance. As a result, quarks cannot exist on their own for long, instead forming colourless bound states called hadrons. Hadrons can be baryons, with three "valence" quarks which each carry a different colour, or mesons, with a valence quark carrying one colour and a valence antiquark carrying its anticolour. The valence quarks in hadrons are accompanied by a "sea" of gluons and quark-antiquark pairs. The gluons and quarks within a hadron are referred to collectively as "partons". "Free" partons produced in high-energy particle interactions will combine with quarkantiquark pairs pulled from the vacuum by the strength of the strong interaction to produce hadrons. These hadrons form "jets" of particles, which appear in a particle physics detector as clusters of energy deposited in the detector. 


\begin{tabular}{|c|c|c|c|}
\hline Particle & Charge & Spin & Mass \\
\hline \multicolumn{4}{|c|}{ Quarks $q$} \\
\hline Up quark $(u)$ & $\frac{2}{3}$ & $\frac{1}{2}$ & 1.5 to $3.3 \mathrm{MeV} / c^{2}$ \\
\hline Down quark $(d)$ & $-\frac{1}{3}$ & $\frac{1}{2}$ & 3.5 to $6.0 \mathrm{MeV} / c^{2}$ \\
\hline Strange quark $(s)$ & $-\frac{1}{3}$ & $\frac{1}{2}$ & $106_{-34}^{+26} \mathrm{MeV} / c^{2}$ \\
\hline Charm quark $(c)$ & $\frac{2}{3}$ & $\frac{1}{2}$ & $1.27_{-0.11}^{+0.07 \mathrm{GeV} / c^{2}}$ \\
\hline Bottom quark $(b)$ & $-\frac{1}{3}$ & $\frac{1}{2}$ & $4.20_{-0.07}^{+0.17} \mathrm{GeV} / c^{2}$ \\
\hline Top quark $(t)$ & $\frac{2}{3}$ & $\frac{1}{2}$ & $171.2 \pm 2.1 \mathrm{GeV} / c^{2}$ \\
\hline \multicolumn{4}{|c|}{ Leptons $l$ and $\nu_{l}$} \\
\hline Electron $(e)$ & -1 & $\frac{1}{2}$ & $0.510998910 \pm 0.000000013 \mathrm{MeV} / c^{2}$ \\
\hline Electron neutrino $\left(\nu_{e}\right)$ & 0 & $\frac{1}{2}$ & $<2 \mathrm{eV} / c^{2}$ \\
\hline Muon $(\mu)$ & -1 & $\frac{1}{2}$ & $105.658367 \pm 0.000004 \mathrm{MeV} / c^{2}$ \\
\hline Muon neutrino $\left(\nu_{\mu}\right)$ & 0 & $\frac{1}{2}$ & $<2 \mathrm{eV} / c^{2}$ \\
\hline $\operatorname{Tau}(\tau)$ & -1 & $\frac{1}{2}$ & $1776.84 \pm 0.17 \mathrm{MeV} / c^{2}$ \\
\hline Tau neutrino $\left(\nu_{\tau}\right)$ & 0 & $\frac{1}{2}$ & $<2 \mathrm{eV} / c^{2}$ \\
\hline \multicolumn{4}{|c|}{ Gauge and Higgs Bosons } \\
\hline Gluon $(g)$ & 0 & 1 & $0 \mathrm{eV} / c^{2}$ (theoretical value) \\
\hline Photon $(\gamma)$ & 0 & 1 & $<10^{-18} \mathrm{eV} / c^{2}$ \\
\hline$W$ boson $\left(W^{ \pm}\right)$ & \pm 1 & 1 & $80.398 \pm 0.25 \mathrm{GeV} / c^{2}$ \\
\hline$Z$ boson $(Z)$ & 0 & 1 & $91.1876 \pm 0.0021 \mathrm{GeV} / c^{2}$ \\
\hline Higgs boson $(H)$ & 0 & 0 & $>114.4 \mathrm{GeV} / c^{2}$ \\
\hline
\end{tabular}

Table 1.1: Particle content of the Standard Model. The particle masses shown are the current experimental limits from Reference [5], except for the theoretical gluon mass. The $\nu$ masses represent a limit on the three $\nu$ mass eigenstates, which are too close together in mass to resolve in mass measurements from tritium $\beta$ decay. 
On the other hand, asymptotic freedom results from the lowered strength of the strong interactions at short distances. It means that quarks or gluons within a hadron can act as free particles in interactions with high momentum exchange. For example, the results of a high-energy collision between two hadrons can be described as a collision of a parton from one hadron and another parton from the second. For example, if a proton with valence quark content $u u d$ and an antiproton with valence quark content $\bar{u} \bar{u} \bar{d}$ interact, the result can be described with the collision of a quark from the proton and an antiquark from the antiproton. Any quarks or gluons produced in the collision will then hadronize. ${ }^{1}$

\subsection{Beyond the Standard Model}

The Standard Model of particle physics has met with considerable success when tested in high-energy particle collisions. The tau neutrino $\nu_{\tau}$, the last lepton to be discovered, was first observed in 2000 [6]. The current world average for the mass of the top quark $t$, the last of the quarks to be discovered, is $171.2 \pm 2.1 \mathrm{GeV} / \mathrm{c}^{2}-$ a precision of $1.2 \%$ [ 5 ]. Of the fundamental bosons postulated in the SM, only the Higgs boson has not yet been discovered. Results from CERN's Large Electron-Positron collider (LEP) and the Stanford Linear Accelerator (SLC) currently set a lower limit on its mass from direct searches of $114.4 \mathrm{GeV} / \mathrm{c}^{2}$ [7], consistent with the $95 \%$ confidence level upper limit of $285 \mathrm{GeV} / \mathrm{c}^{2}$ set by indirect experimental evidence from precision electroweak measurements [8] and with results from the Fermilab Tevatron [9]. The only discovery in recent years to modify the SM is the discovery that neutrinos have a small, but non-zero mass, and that they oscillate between mass eigenstates which are mixtures of their flavour eigenstates [10].

Despite the successes of the SM, it is not believed to be a complete picture of particle physics. There is no unification of the fundamental forces beyond electroweak unifica-

\footnotetext{
${ }^{1}$ The only exception is the top quark, which decays, almost always to a $W$ boson and a $b$ quark, faster than it can hadronize. The signature for $t$ production involves the hadronization products of the resulting $b$.
} 
tion. Indeed, gravity is not even incorporated into the SM. The fact that the number of quark and lepton generations must be identical in order to avoid gauge anomalies in the weak interaction [11] also suggests the possibility of some underlying symmetry relating the quark and lepton sectors. No such symmetry exists in the SM. There is also no symmetry linking the fundamental bosons with the fundamental fermions. Cosmological observations show that unexplained "dark matter" and "dark energy" form the bulk of the universe [12], implying the existence of long-lived, massive particles which only weakly interact with ordinary matter (Weakly Interacting Massive Particles, or WIMPs.) The SM does not, however, contain any WIMP candidates.

If these theoretical questions are to be answered, some sort of new physics beyond the Standard Model (BSM) is needed. Without experimental evidence contradicting the SM, however, any choice of a particular new physics model is arbitrary. A signature-based search can avoid making theoretical assumptions which are neither supported nor ruled out by experimental evidence. By concentrating on an experimental signature which could be produced by new physics, a signature-based search is less biased toward any specific model, and can be applied to several models of BSM physics.

The simplest possible signature-based search is a counting experiment, where the number of events in a dataset passing a particular set of cuts is compared to the number of events expected from SM physics and from one or more BSM models. The interpretation of the results from a counting experiment does not depend on the distribution of any kinematic variables in the data, and can therefore be used to set $95 \%$ cross-section upper limits in exactly the same way for any BSM model, using the formula

$$
\sigma=\frac{N}{A_{s i g} \epsilon \mathcal{L}},
$$

with $\sigma$ being the cross-section limit, $N$ being the event limit, $A_{s i g}$ and $\epsilon$ being the acceptance and efficiency for the signal from a particular BSM model, and $\mathcal{L}$ being the total integrated luminosity. 


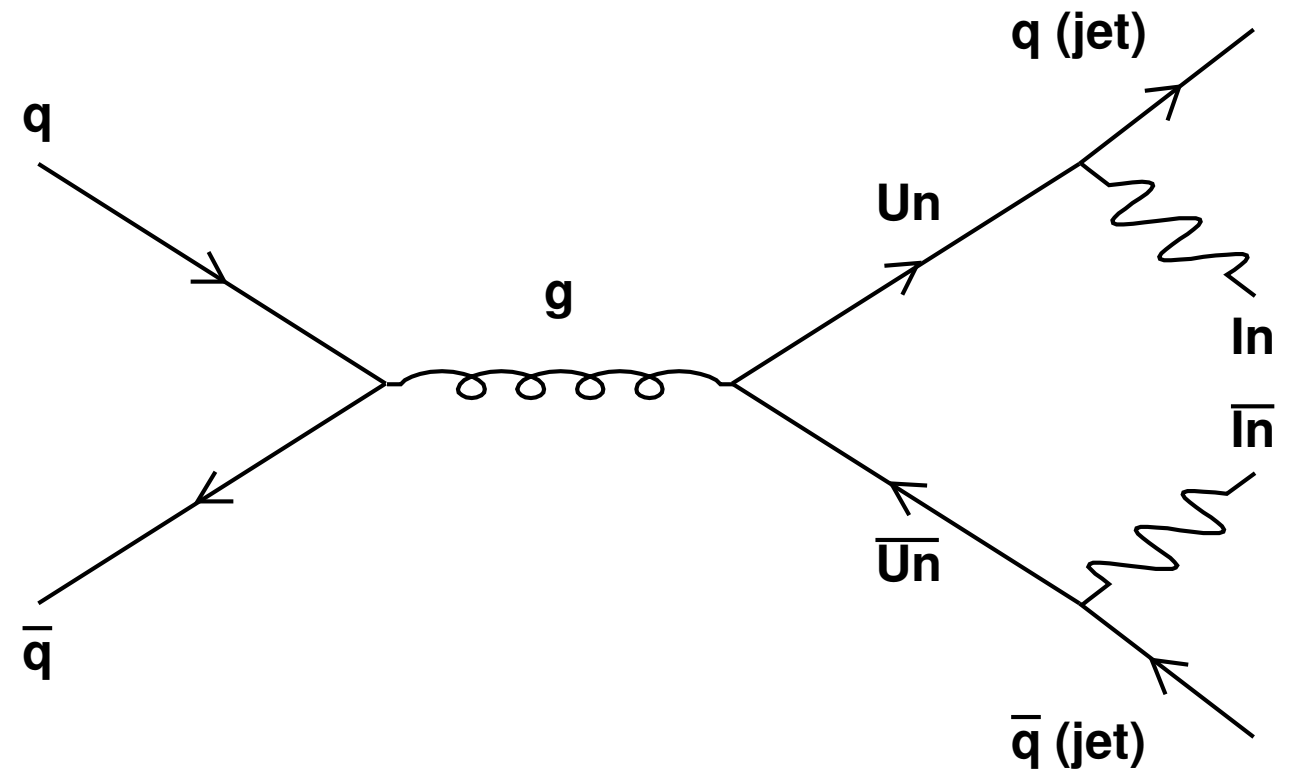

Figure 1.1: Typical Feynman diagram producing a dijet $+\not_{T}$ signature. $U n$ represents a generic unstable particle, and In represents a particle which is invisible to the detector.

In this analysis, events with two jets plus missing transverse energy $\left(E_{T}\right)$ are considered as a possible signature for BSM physics. Figure 1.1 represents a typical Feynman diagram which would produce an exclusive dijet $+E_{T}$ signature at the Tevatron $p \bar{p}$ collider, where this analysis is carried out. For example, if the "invisible" In particle in Figure 1.1 is stable, massive, and non-interacting, it can be a candidate for WIMP dark matter. One class of models which provide a WIMP candidate and could lead to a dijet $+\#_{T}$ signature are minimal supersymmetric (MSSM) models where new particles and antiparticles are produced in pairs [13]. Here, $U n$ would be a squark $\tilde{q}$ while $I n$ could be the lightest neutralino $\tilde{\chi}_{1}^{0}$. Other models of interest could produce a dijet $+\not_{T}$ signature without having a viable dark matter candidate, such as the pair production of unstable leptoquarks $L Q$ which each decay into a quark and an invisible neutrino [14]. 


\subsection{Outline}

As the current energy frontier for particle physics, the Tevatron proton-antiproton collider at Fermilab is the natural place to look for new physics in a variety of signatures. Chapter 2 of this thesis describes the accelerator chain at the Tevatron, and the Collider Detector at Fermilab (CDF) used to collect the data for this analysis.

Chapter 3 discusses the data samples used in this analysis and the triggers used to collect the data. Chapter 4 describes the various cuts which define the two signal regions for this analysis. Chapter 5 explains the method used to determine a data-driven estimate for the SM background to the experimental signature.

Two possible theories of new physics which could lead to a dijet $+\not_{T}$ signature are described in Chapters 6 (for scalar leptoquarks) and 7 (for production of squarks and gluinos in the minimal supersymmetric model). In Chapter 8, the results of the analysis are described, and compared to theoretical expectations from the models described in the preceding chapters. The limits on new physics from these results are also given. Chapter 9 summarizes the results, and discusses possibilities for future studies. 


\section{Chapter 2}

\section{The Tevatron and the Collider Detector at Fermilab}

This analysis was carried out on proton-antiproton collisions produced at the Tevatron, a synchrotron and storage ring complex located at the Fermi National Accelerator Laboratory (Fermilab, or FNAL) in Batavia, Illinois. Data from the collisions was recorded by one of the Tevatron's multi-purpose detectors, the Collider Detector at Fermilab (CDF, or CDF II when specifically referring to the detector as upgraded for the most recent run of the Tevatron.)

This chapter describes the accelerator chain which produces the $p \bar{p}$ pairs in the Tevatron, the CDF detector which detects the results of the $p \bar{p}$ collisions, and the data acquisition systems (DAQ) which collect the data analyzed in later chapters. The description is given for the Run II period which began collecting physics data in 2002, and is ongoing. In many cases, this description represents significant upgrades from the earlier Run I period of the Tevatron, which ran from 1992 to 1996. 


\section{FERMILAB'S ACCELERATOR CHAIN}

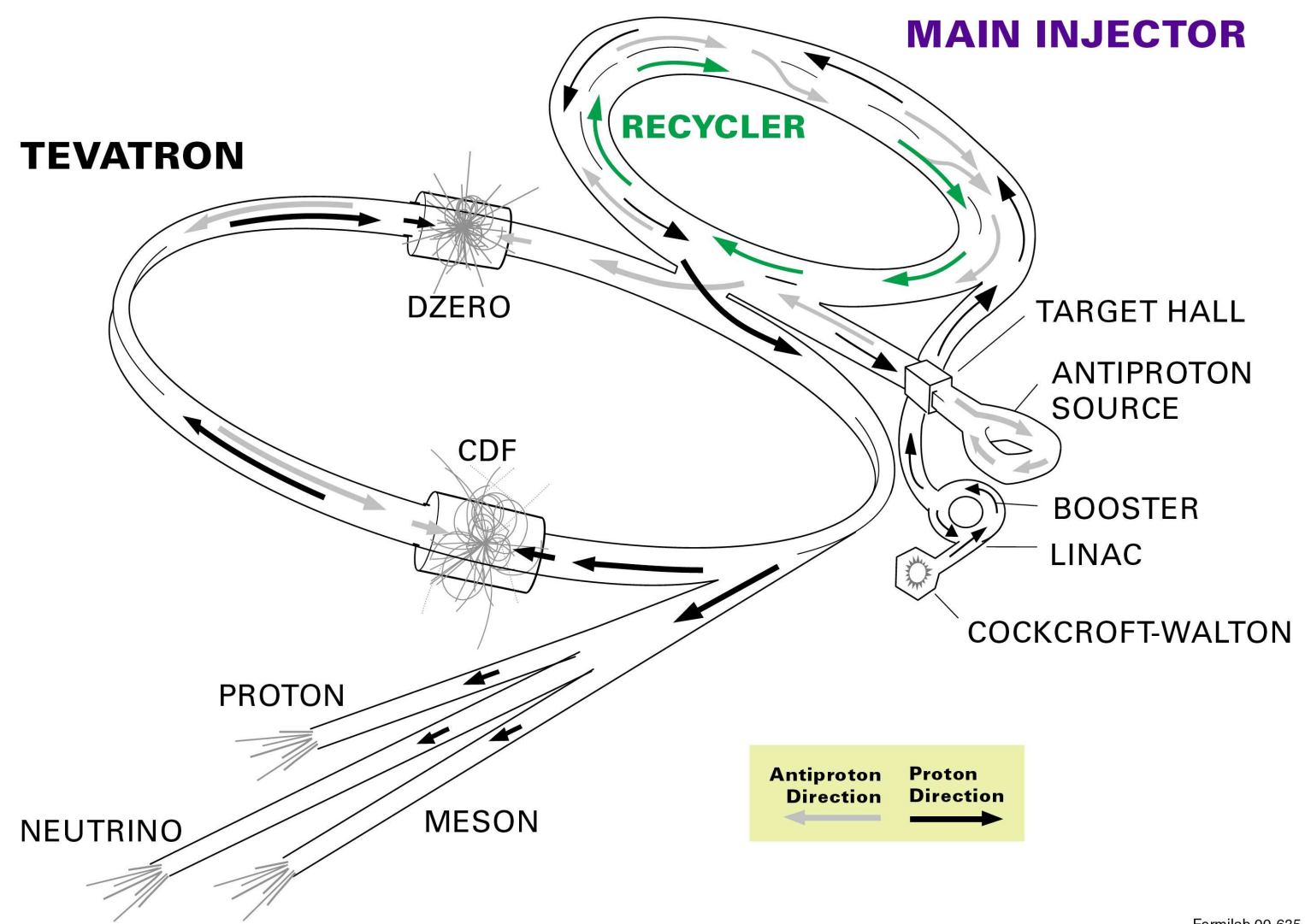

Figure 2.1: The Fermilab Tevatron accelerator chain [15].

\subsection{The Tevatron Accelerator Chain}

At Fermilab, beams of protons and antiprotons $(p$ and $\bar{p})$ are produced, accelerated, and finally collided at a centre-of-mass energy of $1.96 \mathrm{TeV}$ using four main systems: the proton source, the Main Injector synchrotron, the antiproton source, and the Tevatron collider. The accelerator chain is illustrated in Figure 2.1.

\subsubsection{The Proton Source}

The accelerator chain starts with a pair of $H^{-}$ion sources and Cockcroft-Walton electrostatic pre-accelerators. The ion sources are direct-extraction magnetron types, where 
$H^{+}$ions from ionized low-pressure hydrogen gas pick up two electrons when they strike a cesium-coated cathode. Each pre-accelerator boosts the resulting $H^{-}$ions to an energy of $750 \mathrm{keV}$. The ions from both pre-accelerators go on to the linear accelerator system (Linac), which boosts them to $400 \mathrm{MeV}$ [16].

The $400 \mathrm{MeV}$ ions pass through carbon foils, stripping their electrons and making them into $\mathrm{H}^{+}$ions (i.e. protons.) These protons are injected into the Booster, a synchrotron which accelerates them to $8 \mathrm{GeV}[17]$.

\subsubsection{The Main Injector}

The Main Injector synchrotron receives the $8 \mathrm{GeV}$ protons from the Booster. The Main Injector is a versatile device which can accelerate or decelerate protons to several different energies, depending on the mode of operation. In one mode of operation, it will accelerate protons to an energy of $120 \mathrm{GeV}$, then send them to the Antiproton Source (described in subsection 2.1.3) which produces $8 \mathrm{GeV}$ antiprotons. These antiprotons are stored until the accelerator chain enters what is called "Collider Mode" [18].

In Collider Mode, $8 \mathrm{GeV}$ protons in the Main Injector are accelerated to $150 \mathrm{GeV}$ and injected into the Tevatron (described in subsection 2.1.4.) Once the Tevatron has enough protons circulating, the $8 \mathrm{GeV}$ antiprotons from the Antiproton Source are also sent to the Main Injector, where they are also accelerated to $150 \mathrm{GeV}$ and injected into the Tevatron [18].

The Main Injector did not exist during Run I: instead, a synchrotron called the Main Ring was used. Like the Main Injector, it accepted $8 \mathrm{GeV}$ protons from the Booster, sent $120 \mathrm{GeV}$ protons to the Antiproton Source, and sent $150 \mathrm{GeV}$ protons and antiprotons to the Tevatron. This machine shared a tunnel with the Tevatron, which impaired Main Ring operation due to stray magnetic fields from the Tevatron and the detours in the Main Ring's particle path needed to avoid the Tevatron's detectors. Losses from the Main Ring also degraded the data from the detectors at the Tevatron [18]. 


\subsubsection{The Antiproton Source}

In the Antiproton Source, $120 \mathrm{GeV}$ protons from the Main Injector are used to produce antiprotons. The proton beam is focused on a nickel target, producing a shower of secondary particles. A beam of antiprotons with approximately $8 \mathrm{GeV}$ is extracted from the secondary particles. This is an inefficient process - only one or two antiprotons are produced for every $10^{5}$ protons hitting the target - making antiproton production the main limiting factor on the Tevatron's luminosity. The resulting antiproton beam is "cooled" in order to decrease its longitudinal and transverse momentum spread, and stored in the Accumulator synchrotron. Antiprotons are accumulated in this storage ring until there are enough to provide the desired $p \bar{p}$ luminosity in the Tevatron. Once sufficient $\bar{p}$ numbers are present, the beam is sent to the Main Injector to be accelerated to $150 \mathrm{GeV}[19]$.

Another system, the Recycler, is located directly above the Main Injector ring. It can store excess antiprotons produced by the Antiproton Source and sent to the Main Injector, or store antiprotons left in the Tevatron after a Tevatron collision period. In the latter scenario, the Tevatron decelerates the antiprotons to $150 \mathrm{GeV}$ before transferring them to the Main Injector. In either case, antiprotons are decelerated to $8 \mathrm{GeV}$ by the Main Injector and transferred to the Recycler. Antiprotons from the Recycler can be sent back into the Tevatron after being transferred to the Main Injector and accelerated back to $150 \mathrm{GeV}$. The Recycler, like the Main Injector, was constructed as part of the Run II upgrades [18].

\subsubsection{The Tevatron}

The Tevatron synchrotron is loaded with $150 \mathrm{GeV}$ protons and antiprotons from the Main Injector. Being of opposite charge, the protons and antiprotons circle in opposite directions under the influence of the synchrotron's magnets. Both protons and antipro- 
tons are accelerated to an energy of $980 \mathrm{GeV}$ (up from the Run I energy of $900 \mathrm{GeV}$.) The protons and antiprotons then collide at two points in the ring coinciding with the locations of the two general-purpose particle physics detectors at the Tevatron: the CDF and $\mathrm{D} \varnothing$ experiments [20].

In the latter half of Run I (called Run Ib), the average Tevatron luminosity was $\mathcal{L}=$ $1.6 \times 10^{31} \mathrm{~cm}^{-2} \mathrm{~s}^{-1}$. For Run II, a typical luminosity over an order of magnitude greater is possible. The expected average luminosity was $\mathcal{L}=2 \times 10^{32} \mathrm{~cm}^{-2} \mathrm{~s}^{-1}$ [20]. This luminosity was first reached in 2006. During the data taking period for this analysis (between March 23, 2002 and May 13, 2007) peak luminosities approaching $3 \times 10^{32} \mathrm{~cm}^{-2} \mathrm{~s}^{-1}$ were recorded [21].

\subsection{The Collider Detector at Fermilab}

The Collider Detector at Fermilab, pictured in Figure 2.2, is a general purpose particle detector. It can be divided into three main sections: an inner detector which provides charged particle tracking, scintillator-based electromagnetic and hadronic calorimeters, and the outer scintillators and proportional drift chambers which act as muon detectors. The CDF detector has been upgraded from its Run I configuration, as described in Reference [22]. General information on the detector appears in many published CDF II analyses, such as Reference [23].

Figure 2.2 is a cross-sectional view showing half of the CDF detector. CDF is cylindrical in shape, and coaxial with the Tevatron beamline. The tracking detectors are surrounded by a superconducting solenoid coil which produces a uniform $1.4 \mathrm{~T}$ magnetic field. The magnetic field is aligned along the proton beam direction. Outside the solenoid are electromagnetic calorimeters and hadronic calorimeters. The calorimeters are arranged in a projective tower geometry, segmented in azimuth $\phi$ and pseudorapidity $\eta$. Outside the calorimeters are drift chambers used to detect minimally ionizing parti- 


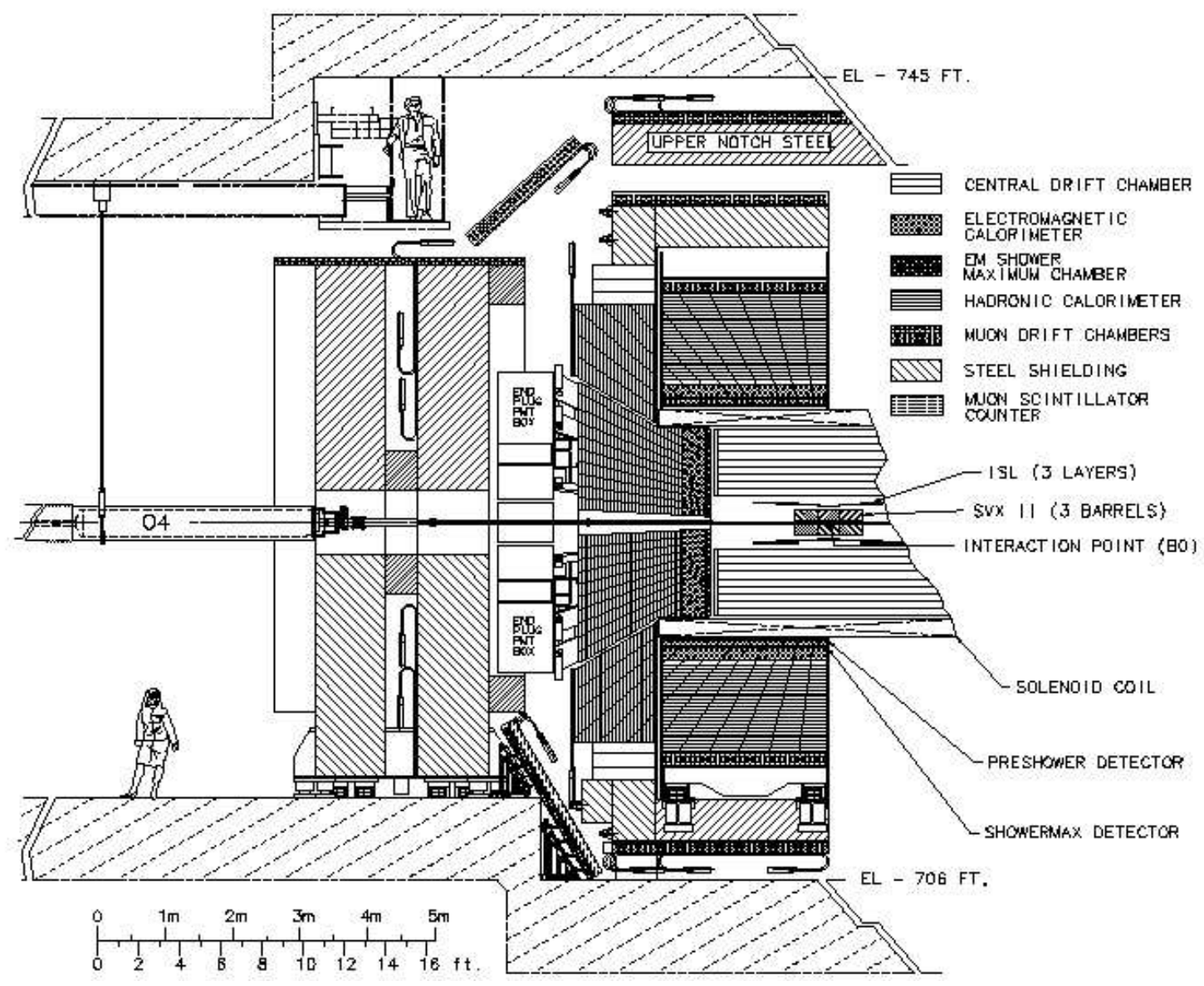

Figure 2.2: Cross-sectional view of half of the Collider Detector at Fermilab, from reference [22]. The axis of the solenoidal magnetic field is along the beam direction.

cles which pass through the calorimeter. These are typically muons, so these systems are referred to as the muon detectors $[22,23]$.

\subsubsection{Co-ordinate System and Related Quantities}

A cylindrical co-ordinate system is defined for CDF, with $r$ being the distance from the beamline, the $z$-axis lying along the beamline, and the proton beam going in the positive $z$ direction. The polar angle $\theta$ is defined relative to the proton direction, and the azimuthal angle $\phi$ is measured from the plane of the Tevatron. Pseudorapidity $\eta=\ln (\tan (\theta / 2))$ is defined with $\theta$ measured from the $z$ where the trajectory crosses the 
beam axis. "Detector" pseudorapidity $\eta_{d e t}$ is calculated by measuring $\theta$ from $z=0 \mathrm{~cm}$, the centre of the detector $[22,23]$.

Tracks in the inner detector, or in the muon chambers, are described in terms of transverse momentum, $p_{T}$, the component of momentum transverse to the beam axis. The momentum vector for a track is described using $p_{T}, \phi$, and $\eta$. Similarly, energy deposition in a calorimeter tower is described in terms of transverse energy $E_{T}=E \sin \theta$, where $E$ is the total energy deposited in the tower. The total $E_{T}$ in an event is given by the sum over all calorimeter towers,

$$
E_{T}=\sum_{i} E_{T}^{i}
$$

where $E_{T}^{i}$ is the transverse energy in the $i$ th tower. The transverse energy of a jet is defined in much the same way (as discussed in Section 4.2 ). A missing $E_{T}$ vector, $\vec{E}_{T}$, is defined as

$$
\overrightarrow{H_{T}}=-\sum_{i} E_{T}^{i} \hat{n}_{i}
$$

with $\hat{n}_{i}$ being a unit vector transverse to the beam axis and pointing at the $i^{\text {th }}$ calorimeter tower. A scalar $E_{T}$ quantity is defined as the magnitude of $\vec{E}_{T}$, and $\phi\left(E_{T}\right)$ is the azimuthal angle of the missing $E_{T}[23]$.

\subsubsection{The Silicon Detectors}

The inner detector for Run II was completely redesigned from its Run I configuration [22]. The original redesign consisted of three systems: the Silicon Vertex Detector (SVX) ${ }^{1}$, the Intermediate Silicon Layers (ISL), and the Central Outer Tracker (COT). A longitudinal view of the original Run II inner detector design is shown in Figure 2.3. The COT will be described in the next subsection.

\footnotetext{
${ }^{1}$ Also called SVX II, to avoid confusion with the Run I detector of the same name.
} 


\section{CDF Tracking Volume}

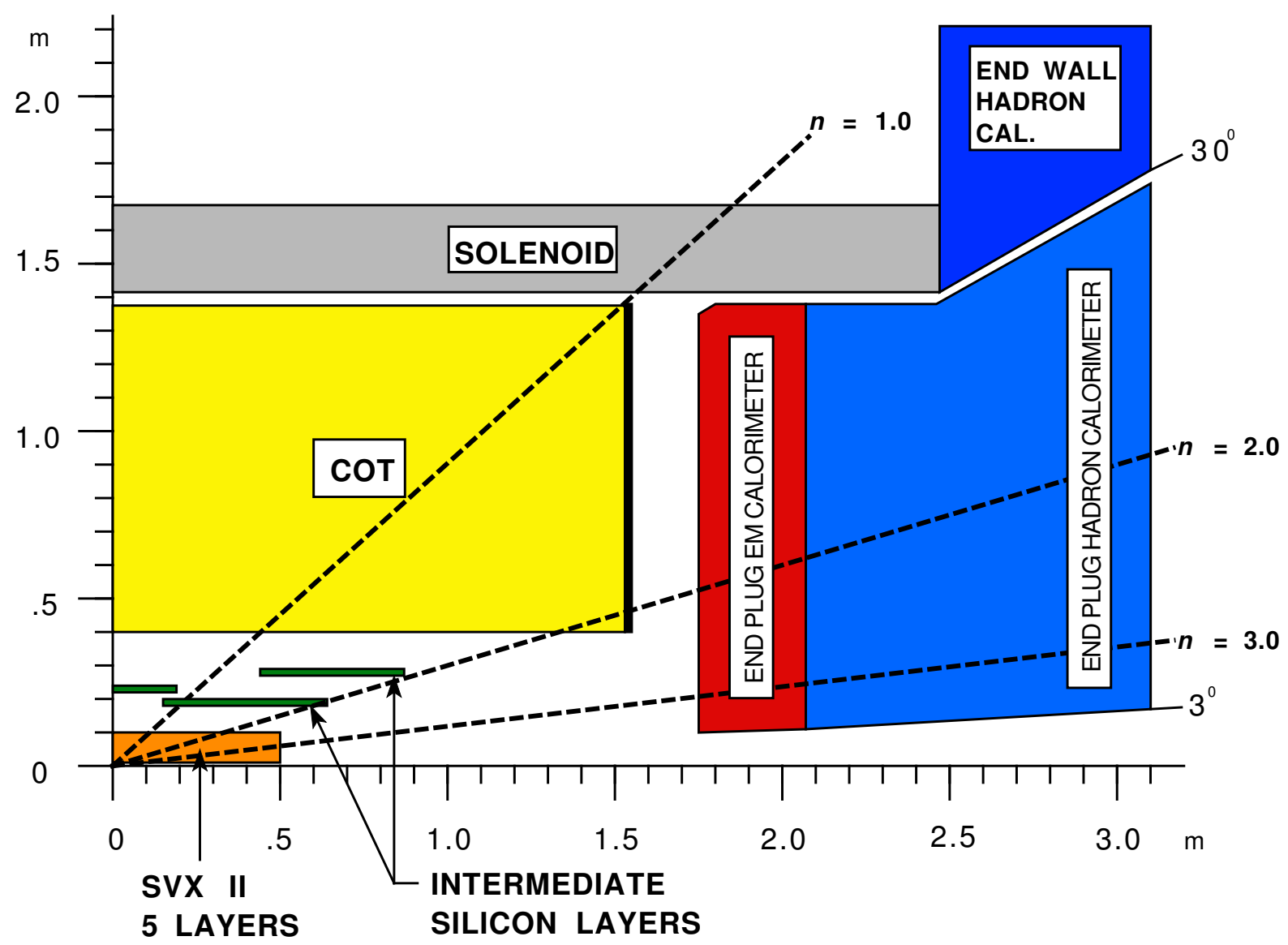

Figure 2.3: Longitudinal view of the CDF inner detector, from reference [22]. L00 and the TOF detector are not shown, as they were not part of the initial design.

The SVX system consists of three cylindrical barrels, located from 2.4 to $10.7 \mathrm{~cm}$ radially outward from the beamline. Each barrel has five layers of double-sided silicon microstrip detectors. Three layers have axial and radial strips, providing an $r-\phi$ stereo measurement, while the other two combine axial with "small angle stereo" strips which are offset by $1.2^{\circ}$. Full SVX coverage extends to $\left|\eta_{\text {det }}\right|<2.0[22]$.

L00 is an additional silicon detector consisting of a detector layer applied directly to the beampipe. L00 was not part of the original Run II design, but was added in order to improve measurements of the distance between track vertices and the beam axis (also called the impact parameter of a track.) L00 is a layer of single-sided silicon 
detectors, designed to be radiation-resistant. Being so close to the beamline, it covers $\left|\eta_{\text {det }}\right|<4.0[24]$.

The ISL system comprises three layers of double-sided silicon detectors located between $r=20 \mathrm{~cm}$ and $r=28 \mathrm{~cm}$. Two forward layers at $r=20$ and $28 \mathrm{~cm}$ provide tracking for $1.0<\left|\eta_{\text {det }}\right|<2.0$, with a central layer at $r=22 \mathrm{~cm}$ for the central $\left|\eta_{\text {det }}\right|<1.0$ region. The ISL silicon detectors combine axial with "small angle stereo" strips which are offset by $1.2^{\circ}[22]$.

Commissioning for the entire silicon system was not completed until June 2002, meaning that early data from Run II was taken without full information from the silicon system [25].

\subsubsection{The Central Outer Tracker}

The COT, labeled as the "Central Drift Chamber" in Figure 2.2, is an open-cell drift chamber located radially outward from the silicon detectors. It consists of eight "superlayers", located at an $r$ between 40 and $132 \mathrm{~cm}$. Each superlayer contains twelve layers of sense wires alternating with twelve layers of potential wires. Four of the superlayers have wires strung parallel to the beam axis, and are called "axial layers." They alternate with "stereo layers", where the wires are strung at $\pm 2^{\circ}$ angles from the beam axis, allowing particle tracking along the $z$-axis. Combined with the known $r$ for each layer and the $\phi$ from the axial layer hits, three-dimensional tracking is possible. The innermost superlayer is a stereo layer [22, 23].

The COT has 30,240 readout channels, and covers $\left|\eta_{\text {det }}\right|<1.0$. Tracks in the COT can be measured with a single-hit resolution of $180 \mu \mathrm{m}$, which is the standard deviation of the residuals between a reconstructed track and its consitituent hits in the COT. The transverse momentum of the tracks is determined from $p_{T}=0.3 q B r_{c} \mathrm{GeV} / c$, where $q$ is the total particle charge (in terms of multiples of the magnitude of the electron charge), $B$ is the solenoidal magnetic field $(1.4 \mathrm{~T})$, and $r_{c}$ is the track's radius of curvature in 
metres [23].

Just outside the radius of the COT is the time-of-flight (TOF) detector. Like L00, the TOF detector was not part of the initial Run II CDF upgrade, but was added later to improve particle identification by providing time-of-flight information for particles travelling through the inner detector. It consists of 216 scintillator bars, each running the length of the COT. The TOF detector provides a $100 \mathrm{ps}$ time resolution for particles with $p<1.6 \mathrm{GeV} / c[26]$.

\subsubsection{The Calorimeters}

Calorimetry at CDF uses several sampling scintillator calorimeters with tower-based projective geometry. The energy of photons and electrons is measured by the inner sections of each tower, which use lead as the absorber. The outer sections of each tower measure energy deposited by hadrons, and use steel as the absorber. Each tower is read out by two photomultiplier tubes (PMTs), one at the low $\phi$ end of the tower and one at the high $\phi$ end. Differences in timing between the readout of the two PMTs allow a more precise determination of the $\phi$ of an energy deposition. The calorimeter is divided into two main sections: the central barrel calorimeters cover $\left|\eta_{\text {det }}\right|<\sim 1.0$, while the plug calorimeters cover $\sim 1.1<\left|\eta_{\text {det }}\right|<\sim 3.6$. The central calorimeters are unchanged from Run I, while the forward calorimeters are new for Run II [22, 23].

There are three central calorimeter subsystems: the Central Electromagnetic Calorimeter (CEM), the Central Hadronic Calorimeter (CHA), and the Endwall Hadronic Calorimeter (WHA). Each is divided into towers which cover an $\eta_{\text {det }}$ range of 0.1 and a $\phi$ range of $15^{\circ}=\pi / 12 \mathrm{rad}$. The CEM covers $\left|\eta_{\text {det }}\right|<1.1$, the CHA covers $\left|\eta_{\text {det }}\right|<0.9$, and the WHA completes the CHA's coverage and provides additional forward coverage by

covering $0.6<\left|\eta_{\text {det }}\right|<1.3[22,23]$. There are two important regions of the central calorimeter which are not covered by calorimeter towers. These uninstrumented regions are the "chimney" $\left(0.5<\eta_{\text {det }}<1.0,1.05 \mathrm{rad}<\phi<1.66 \mathrm{rad}\right)$ which is taken up by 
cables for detector electronics and cryogenic utilities for the solenoid, and the "crack" where the east and west halves of the calorimeter meet $\left(\left|\eta_{\text {det }}\right|<0.05\right.$. $)$

The plug calorimeter contains the Plug Hadronic Calorimeter (PHA) and the Plug Electromagnetic Calorimeter (PEM). The PEM covers $1.1<\left|\eta_{\text {det }}\right|<3.6$ while the PHA covers $1.3<\left|\eta_{\text {det }}\right|<3.6$. Like the central calorimeters, these are divided into projective readout towers segmented in $\eta$ and $\phi$. The size of the tower varies with $\eta_{d e t}$, from 0.1 in $\eta_{\text {det }}$ and $7.5^{\circ}$ in $\phi$ at $\left|\eta_{\text {det }}\right|=1.1$ to 0.5 in $\eta_{\text {det }}$ and $15^{\circ}$ in $\phi$ at $\left|\eta_{\text {det }}\right|=3.64[22,23]$.

The energy resolutions are determined from testbeam data, where calorimeter modules were exposed to electron or charged pion beams before installation. The resolution is expressed in terms of $\sigma(E) / E$ for the PHA and PEM, and in terms of $\sigma\left(E_{T}\right) / E_{T}$ for the WHA, CHA, and CEM. For high-energy electrons and photons in the electromagnetic calorimeters, the energy resolution is $13.5 \% / \sqrt{E_{T}} \oplus 1.5 \%$ for the CEM and $16 \% / \sqrt{E} \oplus 1 \%$ for the PEM (with $E$ and $E_{T}$ in $\mathrm{GeV}$ ). For the hadronic calorimeters, the energy resolution for charged pions which do not interact with the electromagnetic calorimeters is $50 \% / \sqrt{E_{T}} \oplus 3 \%$ for the CHA, $75 \% / \sqrt{E_{T}} \oplus 4 \%$ for the WHA, and $80 \% / \sqrt{E} \oplus 5 \%$ for the PHA [27].

Both the CEM and PEM have proportional chambers included at a distance roughly corresponding to the maximum shower ionization density region for electrons passing through the calorimeter. These are called the Central and Plug ShowerMax detectors (CES and PES). These systems are used to measure the lateral profile for an electron shower and extract the location of the incident particle. The innermost layers of the CEM and PEM also have scintillator tiles used as pre-shower detectors (called CPR in the barrel and PPR in the plug.) These systems provide additional information useful in electron identification $[22,23]$. The gaps in $\phi$ between the CEM's towers are covered by the Central Crack Detectors (CCR) which combine with the CPR to cover the inner rim of the CEM [28]. 


\subsubsection{The Muon Detectors}

The muon detector system includes four sets of drift chambers: the central muon detector $(\mathrm{CMU})$, the central muon upgrade detector (CMP), the central muon extension detector $(\mathrm{CMX})$, and the barrel muon upgrade detector (BMU). The CMU is the same system used in Run I, while the CMP and CMX are upgraded to provide additional coverage, and the BMU is completely new for Run II $[22,23]$. For the early part of Run II, however, the upgraded systems were still being commissioned, so muon measurements from those periods use the Run I components only [23].

The CMU is cylindrical, and covers $\left|\eta_{\text {det }}\right|<0.6$. The CMP provides additional coverage for the same $\eta_{\text {det }}$ range. It is located outside the CMU, forming a box-shaped structure. An additional $60 \mathrm{~cm}$ of steel absorber is located between the CMU and CMP, and a set of scintillators are on the outer surfaces of the CMP. The CMX consists of two truncated cones on either end of CDF, covering $0.6<\left|\eta_{\text {det }}\right|<1.0$, with scintillators on the inner and outer surfaces. The bottom $90^{\circ}$ of the CMX has a modified geometry due to the presence of the detector hall's floor: this portion of the CMX is called the "miniskirt." The east half of the CMX also has no coverage over $70^{\circ}<\phi<105^{\circ}$, in order to make room for the solenoid cryogenics. This region is called the "keystone". The western part of the CMX does cover the keystone region. Both the miniskirt and west keystone portions of the CMX are new for run II. The BMU is made up of two cylindrical detectors covering $1.0<\left|\eta_{\text {det }}\right|<1.5$, with scintillator tiles on the outer surface. Two additional pinwheel-shaped arrays of scintillators associated with forward muon detection are used, one at either end of $\mathrm{CDF}[22,23]$.

Muons in this analysis are found by matching tracks in the inner detector to patterns of hits, called "stubs", in the muon detector. More information on how muons are identified using the muon detectors can be found in Subsection 3.2.1. 


\subsubsection{The Cherenkov Luminosity Counters}

Instantaneous and integrated luminosity for $\mathrm{CDF}$ is measured using a system of gas Cherenkov counters: the Cherenkov Luminosity Counters (CLCs). Two CLC modules are used, one at each end of the detector. They cover the region between $3.6<\left|\eta_{\text {det }}\right|<4.6$. Each module contains 48 conical gas Cherenkov counters, arranged around the beam pipe in three concentric layers of 16 counters each. The counters are made of aluminized Mylar, and are filled with isobutane gas. A light collector on the end of each counter gathers Cherenkov light produced from particles passing through the CLC into PMTs. Due to the high $\left|\eta_{\text {det }}\right|$, most of the particles detected by the CLC are inelastic $p \bar{p}$ events [23].

Total integrated luminosity $L$ is derived from the rate of inelastic $p \bar{p}$ events measured by the CLC $\left(R_{p \bar{p}}\right)$, the CLC acceptance $\left(\epsilon_{C L C}\right)$, and the inelastic $p \bar{p}$ cross-section at 1.96 $\mathrm{TeV}\left(\sigma_{i n}\right)$, using the expression: [23]

$$
L=\frac{R_{p \bar{p}}}{\epsilon_{C L C} \sigma_{i n}}
$$

Measurement of $\epsilon_{C L C}$ gives $60.2 \pm 2.6 \%$. The Run I result for $\sigma_{i n}$ at $\sqrt{s}=1.8 \mathrm{TeV}$ is scaled up to $1.96 \mathrm{TeV}$ to get $\sigma_{i n}=60.7 \pm 2.4 \mathrm{mb}$. The relative uncertainties on these two values are added in quadrature to a $2.0 \%$ uncertainty from detector instability and a $1.5 \%$ uncertainty from detector calibration to get a total luminosity uncertainty of $6.0 \%$ [23].

\subsection{The Triggers and Data Acquisition Systems}

CDF has a three-stage trigger system. It is designed to record only "interesting" events, since the collision rate is much higher than the rate at which event information can be recorded. Each successive stage of the trigger system is more selective, runs more slowly than the previous stage, and receives data at a slower rate due to the filtering from the previous stage. The first two levels of the trigger system are run on customized electronic hardware, while the third level uses software running on general-purpose computers. 


\section{Dataflow of CDF "Deadtimeless" Trigger and DAQ}

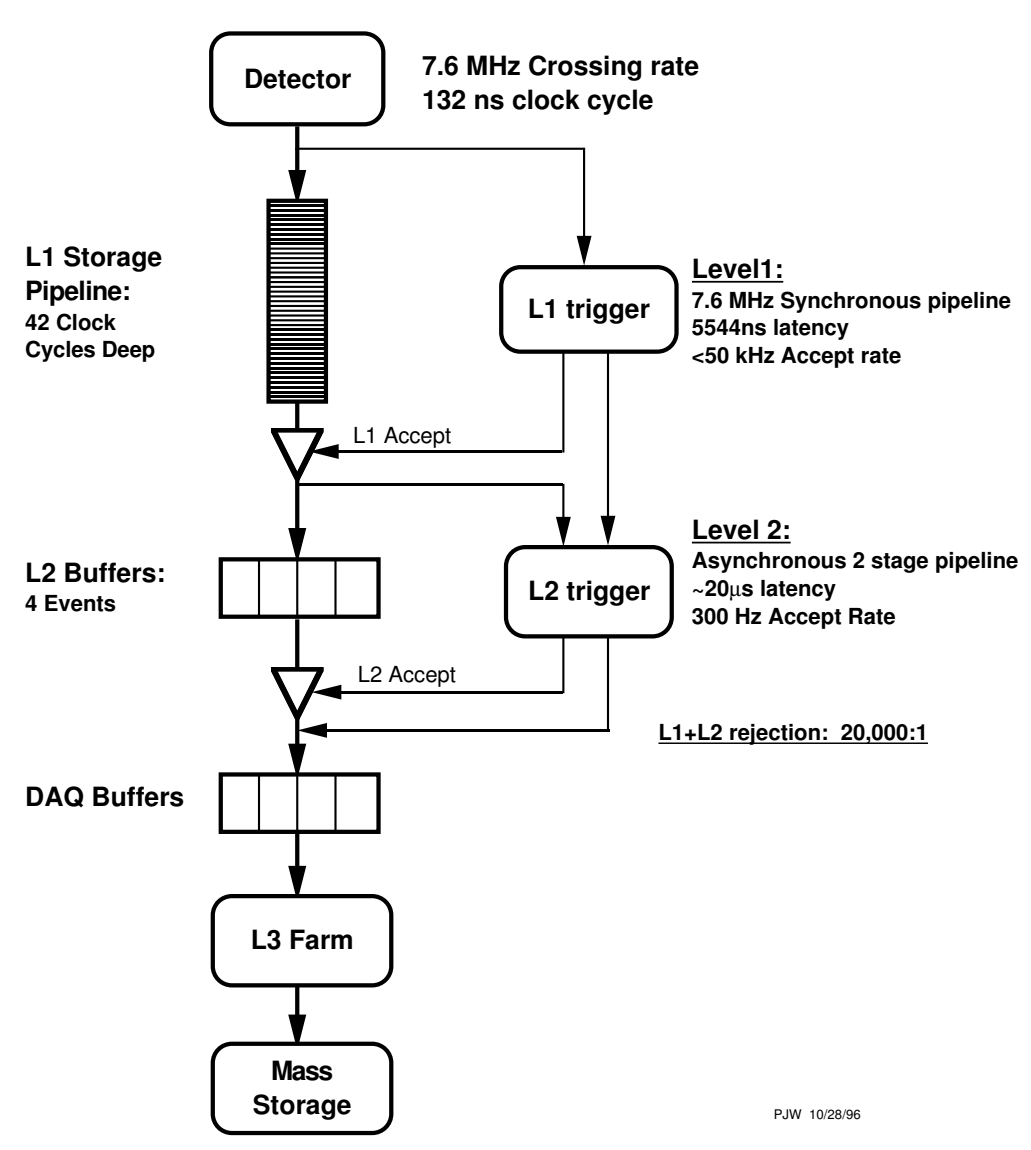

Figure 2.4: Block diagram of the data flow at CDF, from reference [22].

Figure 2.4 shows a diagram of the data flow from the detector, through the three trigger stages, and into the data storage systems.

The first section of the trigger system is referred to as Level 1, or L1. In the L1 hardware, jets and electrons are identified from single calorimeter towers with energy depositions passing a given threshold. The total $E_{T}$ and $E_{T}$ are also calculated from all calorimeter towers, assuming a collision vertex $z=0 \mathrm{~cm}$. Hits in the COT are converted into tracking information by the eXtremely Fast Trigger (XFT), and are also sent to L1. Similarly, muon candidates are identified based on hits in the muon systems. Based on the number of physics objects (jets, tracks, muon candidates, or electron candidates) 
found and the total $E_{T}$ and $E_{T}$ in the event, the event may or may not pass the L1 triggers. Information for events passing L1 are stored in one of four buffers before being sent on to the Level 2 (L2) trigger systems, allowing more events to be analyzed by L1 while waiting for L2 to analyze a previous event [22, 23].

L2 is the first level where information from the whole detector, including the CES and the silicon detectors, is incorporated. The Silicon Vertex Trigger (SVT) accepts information from the silicon detectors and the COT information from the XFT, and finds track candidates with displaced vertices. Dedicated calorimeter L2 hardware merges nearby towers with energy deposits into clusters. CES information is also sent to L2 to help identify low- $E_{T}$ electrons. The SVT, calorimeter, CES, and L1 information for an event are sent to L2 processors. The L2 processor board can process one event while reading in another, thus decreasing deadtime where no new information can be processed. As in L1, algorithms which check the number and type of physics objects are used to determine which events are selected to go to the next level of the trigger system [22, 23].

Once the L2 hardware has selected events, they are read out of the front-end detector buffers into the Level 3 (L3) processing farm. This is a farm of some 300 commercial dual-processor computers running the Linux operating system and a speed-optimized version of the offline reconstruction code. Each processor fully analyzes one event at a time. At L3, full three-dimensional track reconstruction, including information from the entire inner detector, is made. The resultant tracks can be matched to clusters in the electromagnetic calorimeter and to stubs in the muon systems, allowing electron and muon identification. Events passing an L3 trigger after a full analysis are sent to a data logging system to be stored $[22,23]$. 


\section{Chapter 3}

\section{Data Samples}

Three main data samples are examined in this analysis. Two of the samples, the high- $p_{T}$ electron and muon samples, are used to characterize the background in the $\mathscr{E}_{T}+$ dijet signature from electroweak processes. The data sample used to search for the $E_{T}+$ dijet signal is collected using the MET45 trigger path, which requires $45 \mathrm{GeV}$ of $\not_{T}$. The efficiency of the MET45 trigger is examined using the high- $p_{T}$ muon sample.

\subsection{Run Lists and Luminosities}

The data taken at CDF during Run II is divided into several "run periods" which cover different periods of operation. The data used in this analysis is divided into thirteen run periods, numbered sequentially from 0 to 12 . Each run period is divided into many smaller, individually numbered "runs" which represent much shorter periods of data taking. The run numbers used in this analysis range from 141544 to 241664 . Some of these runs contain data other than $1.96 \mathrm{TeV} p \bar{p}$ collisions with a standard trigger table: they may contain cosmic ray tests without beam in the Tevatron, calibration tests using special trigger tables, or test runs with some parts of the detector in standby mode. Each run is further subdivided into numbered run sections. 
As data is taken, the conditions for each run are recorded, including the state of the detector components and the trigger table used. Various "good run lists" are recorded offline, which record which runs or run sections contain data from $1.96 \mathrm{TeV} p \bar{p}$ collisions, using the proper trigger tables, and with all relevant detector systems operating properly. Depending on which detector systems are needed for a given analysis, different good run lists exist. For example, a measurement using muons or a muon trigger would use a good run list where the muon systems are operating properly throughout. A data sample from a good run list which includes runs where the muon systems were not working would be acceptable for a measurement which does not use muons.

The most inclusive good run list used in this analysis is the "QCD/no silicon" list, which includes all standard physics runs where the calorimeter and the COT were working properly. This would be the appropriate run list to use when analyzing the MET45 data sample. However, between runs 178745 and 186598, there was a problem with the L2 trigger for the MET45 path - the L2 $\not_{T}$ threshold was set to $100 \mathrm{GeV}$. These runs, covering approximately $100 \mathrm{pb}^{-1}$, were removed from the MET45 good run list. The resulting sample has $2039 \mathrm{pb}^{-1}$ of data ${ }^{1}$.

For the high- $p_{T}$ muon sample, this analysis uses the CDF good run list for the muon triggers, which is more restrictive than the "QCD/no silicon" good run list as it requires that the CMU, CMP, and CMX muon systems be functioning. For run numbers $<150145$, when the CMX was not fully operational, a run only needs the CMP and CMU to be working to be marked as good. The total integrated luminosity for this sample is $2019 \mathrm{fb}^{-1}$.

The good run list for the high- $p_{T}$ electron sample adds the condition that the shower maximum detector in the calorimeter is working to the "QCD/no silicon" good run list. It has an integrated luminosity of $2148 \mathrm{pb}^{-1}$.

\footnotetext{
${ }^{1}$ All integrated luminosities quoted in this thesis have a $6.0 \%$ relative uncertainty (see Subsection 2.2.6).
} 


\subsection{The High- $p_{T}$ Lepton Samples}

This analysis calculates the electroweak backgrounds to the dijet $+\not_{T}$ signal using measurements from real data whenever possible, thus avoiding such uncertainties as those arising from differences in the jet energy scale between data and Monte Carlo. Standard CDF high- $p_{T}$ lepton (i.e. muon and electron) data samples are used.

The high- $p_{T}$ lepton samples contain data using standard trigger paths, which are sets of level 1,2, and 3 trigger conditions. As with all trigger paths at CDF, each successive level of the triggers applies more stringent cuts. With the increased instantaneous luminosity in later run periods, the level 1 and level 2 cuts are made more stringent in order to keep the rate of data transferred to the next levels low enough for the hardware or software to process them. The level 3 cuts, however, remain unchanged throughout the data-taking period.

\subsubsection{The High- $p_{T}$ Muon Sample}

Two trigger paths are used to determine which events go into the high- $p_{T}$ muon sample: L3_MUON_CMUP18 and L3_MUON_CMX_18. These trigger paths find muons in the CMU and CMP systems, and muons in the CMX systems respectively. The requirements

for these trigger paths search for signatures consistent with minimum ionizing particles such as muons, which leave tracks in the inner detector and the muon chambers without making significant energy deposits in the calorimeters. The trigger conditions at each level are as follows [23, 29, 30]:

- For level 1:

- For CMU or CMP muons, hits in the CMU and in the CMP which are consistent in $\phi$ with one another must match a COT track with $p_{T}>4 \mathrm{GeV} / c$.

- Similarly for CMX muons, hits in the CMX must match a COT track with 
$p_{T}>8 \mathrm{GeV} / c$. In later parts of the data collection period, a matching hit in the CSX is also required.

- For level 2:

- For early runs, no further conditions are set at level 2.

- The CMU and CMP muon trigger in later runs must have at least one COT track with $p_{T}>8 \mathrm{GeV} / c$. This L2 trigger is not required to match the CMU or CMP hits used in the L1 trigger. At later run periods, this track $p_{T}$ threshold goes up to $15 \mathrm{GeV} / c$.

- Similarly for the CMX trigger, a track with $p_{T}$ greater than $10 \mathrm{GeV} / c$, or later $15 \mathrm{GeV} / c$, is required in later run periods.

- For the CMU/CMP and CMX muon triggers at level 3, a reconstructed COT track must match the reconstructed stub in either the CMU and CMP or in the CMX, respectively, and have a $p_{T}>18 \mathrm{GeV} / c$.

\subsubsection{The High- $p_{T}$ Electron Sample}

The events in the high- $p_{T}$ electron sample are collected using the trigger path ELECTRON_CENTRAL18. The cuts in this trigger path are designed to find events where significant energy is deposited in the electromagnetic calorimeter, with a high- $p_{T}$ track in the COT pointing toward the energy deposition. Such a signature is consistent with a charged particle like an electron, which will leave a track in the inner detector then deposit almost all of its energy in the electromagnetic calorimeter. In this trigger path [23, 29]:

- At level 1, at least one tower in the CEM must have an energy deposition above $8 \mathrm{GeV}$. The ratio of hadronic to electromagnetic energies in that tower must satisfy $E_{\mathrm{HAD}} / E_{\mathrm{EM}}<0.125$. A COT track with $p_{T}>8 \mathrm{GeV} / c$ must point to this tower. 
- At level 2, a clustering algorithm adds adjacent "shoulder" towers to the "seed" tower from level 1 . The total $E_{T}$ of the cluster must be above $16 \mathrm{GeV}$, with $E_{\mathrm{HAD}} / E_{\mathrm{EM}}$ still below 0.125 , and a COT track with $p_{T}>8 \mathrm{GeV} / c$ must be reconfirmed.

- At level 3, the cluster must have $E_{T}>18 \mathrm{GeV}, E_{\mathrm{HAD}} / E_{\mathrm{EM}}<0.125$, and a COT track with $p_{T}>9 \mathrm{GeV} / c$.

\subsection{The MET45 Data Sample}

In order to examine events with high $\not_{T}$, the MET45 trigger pathway is chosen. MET45 is an inclusive $E_{T}$ trigger, which does not check for the presence of any other physics objects in the event. The cuts in this analysis identifying the exclusive dijet $+E_{T}$ signature are carried out with offline cuts.

The trigger path for this sample is as follows [29]:

- At level $1, E_{T}$ must be greater than $25 \mathrm{GeV}$.

- At level 2 , for early runs, no new requirements appear. In later runs with higher instantaneous luminosity, the $\not_{T}$ must be greater than $35 \mathrm{GeV}$. This keeps the rate of data transmission to the level 3 system low enough for the level 3 systems to handle.

- At level $3, E_{T}$ must be greater than $45 \mathrm{GeV}$.

\subsubsection{MET45 Trigger Efficiency}

In order to determine the efficiency of the MET45 trigger path, the high- $p_{T}$ muon sample is used once again. Since the muon candidates for the trigger do not depend on any calorimeter information, this sample is unbiased with respect to the MET45 trigger. 
In order to find the MET45 trigger efficiency, high- $p_{T}$ muon data is divided into bins of width $5 \mathrm{GeV}$ in $E_{T}$. The $E_{T}$ here is corrected to take into account jet energy corrections, as explained in Section 4.2. The proportion of the events in each bin which pass the MET45 trigger path is plotted in Figure 3.1. A statistical uncertainty is computed for each bin using the formula

$$
\Delta E=\frac{\sqrt{N E(1-E)}}{N},
$$

where $N$ is the total number of events in the bin, and $E$ is the fraction of events passing the trigger. Figure 3.1 shows that the trigger efficiency begins to plateau at about $99 \%$ after the $80 \mathrm{GeV}<E_{T}<85 \mathrm{GeV}$ bin. For some of the higher bins, the efficiency as measured drops significantly. This is because when a very large amount of energy is deposited in a calorimeter tower, it may saturate and under-report the energy, resulting in an underestimation of the $E_{T}$ in the event. This means that while a corrected $E_{T}$ calculated after correcting jet energies may push the $\mathscr{E}_{T}$ above a given threshold, online reconstruction may not allow the event to pass the MET45 trigger. The large uncertainties in some of these high- $\not_{T}$ bins are due to the low numbers of events in these bins. For example, one or two events may pass the trigger out of three total events in the bin. Other high- $\not_{T}$ bins have no measurable statistical error, because all of the small number of events in those bins passed the MET45 trigger.

The important quantity when determining efficiency is not the bin-per-bin trigger efficiency, but the cumulative efficiency for all events above a given $\not_{T}$ threshold. Figure 3.2 shows the cumulative efficiency for the MET45 trigger. It shows that for events passing a $\not_{T}$ cut between $80 \mathrm{GeV}$ and $110 \mathrm{GeV}$, the trigger is $99 \%$ efficient. At higher $\not_{T}$ thresholds, the saturation effect begins to lower the cumulative efficiency, as a larger fraction of events with $E_{T}$ reconstructed offline to be above the threshold have a $E_{T}$ in the online reconstruction which is too low to pass the trigger. The statistical uncertainty in each bin is calculated using Equation 3.1. 


\section{Efficiency for MET45 Trigger (high-pt muons)}

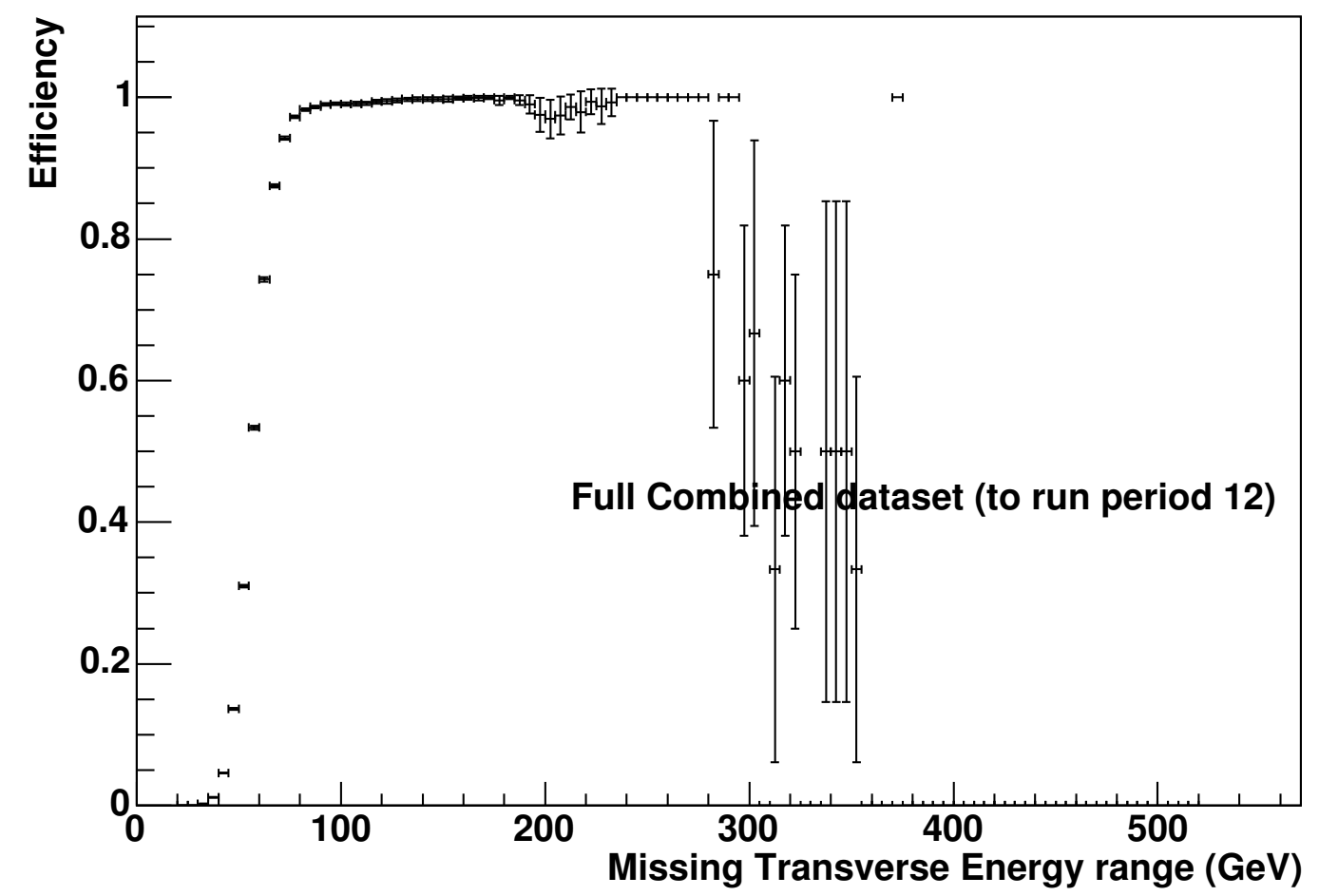

Figure 3.1: Bin-per-bin efficiency distribution of the MET45 trigger as a function of the reconstructed $E_{T}$. The vertical error bars are statistical.

Since the $\#_{T}$ distributions for a particular signal may vary, the cumulative trigger efficiency from the muon sample will not be the correct efficiency to use when setting limits on new physics. Instead, the bin-per-bin distribution in Figure 3.1 must be convoluted with the $\#_{T}$ distribution from a Monte Carlo simulation of the signal. Then, the cumulative trigger efficiency can be determined. In Chapter 8, this method will be used in order to set limits. This step is unnecessary when considering SM backgrounds, however: this is because the muon sample is primarily $W^{-} \rightarrow \mu \bar{\nu}_{\mu}$ events, which have a $\not_{T}$ distribution similar to the $W^{-} \rightarrow l \bar{\nu}_{l}$ or $Z \rightarrow l \bar{l}$ plus jets events which, as shown in Chapter 5, make up the majority of the SM background in this analysis. ${ }^{2}$

\footnotetext{
${ }^{2}$ In this thesis, any mention of $W^{-} \rightarrow l \bar{\nu}_{l}$ events should be understood to include $W^{+} \rightarrow \bar{l} \nu_{l}$ events as well.
} 


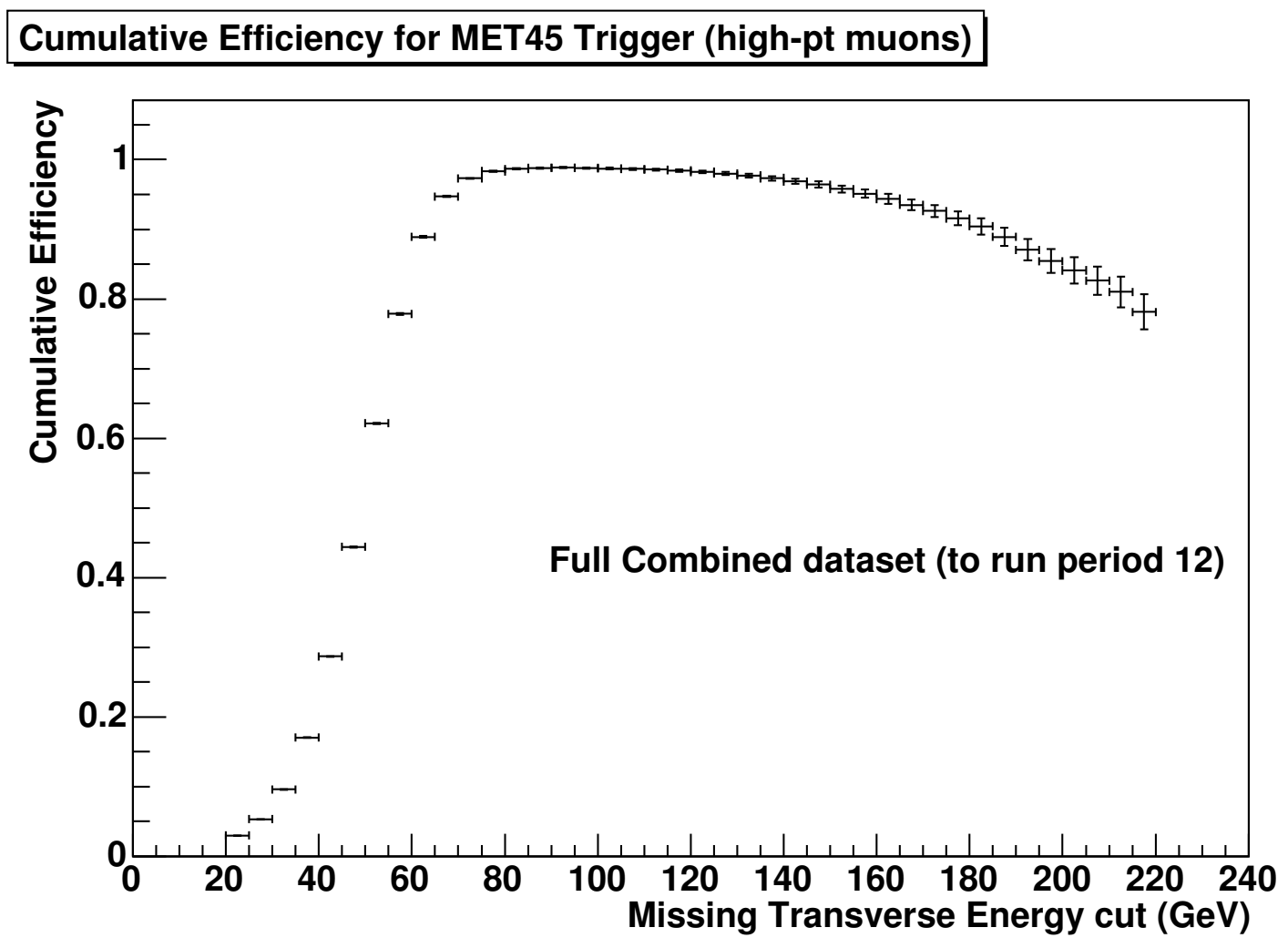

Figure 3.2: Cumulative efficiency distribution for the MET45 trigger as a function of the reconstructed $\#_{T}$. The vertical error bars are statistical. 


\section{Chapter 4}

\section{Selection Cuts}

An exclusive dijet $+\not_{T}$ signature is used in this analysis. First, the jet definition used in the analysis is discussed. The cuts used to remove non-collision events from the data sample are explained. Two kinematic regions are defined in this analysis, a "low" region with kinematic cuts motivated by trigger efficiency, and a "high" region with tighter cuts motivated by the need to keep the uncertainty in the SM background prediction at the 10-15\% level. The total uncertainties on the background prediction for the two sets of cuts, as calculated in Chapter 5, turn out to be $6 \%$ for the low kinematic region and $14 \%$ for the high kinematic region. Both sets of cuts have trigger efficiencies above $98 \%$ for the new physics signal models examined.

\subsection{Isolated Track Veto}

The signature for this analysis is an exclusive dijet $+\not{ }_{T}$ signal, with no other objects present in the final state. In order to get an exclusive dijet $+\not \not_{T}$ signal, events with an isolated track are excluded. This veto reduces the number of $W^{-} \rightarrow l \bar{\nu}_{l}+$ jets or $Z \rightarrow l \bar{l}+$ jets events in the signal region by removing events with muons. It also removes events with electrons which produce jets that pass the jet EM fraction cut (discussed in Subsection 4.2.2), or which lead to jets in the uninstrumented regions of the calorimeter. 
In order to be considered an isolated track, a track must first satisfy the following requirements which make it a well-measured high-momentum track:

- Five or more hits in three or more axial COT layers

- Five or more hits in two or more stereo COT layers

- Track $p_{T}>10.0 \mathrm{GeV} / c$

- $z_{0}$ within $10 \mathrm{~cm}$ of the event vertex

- $\left|d_{0}\right|<0.5 \mathrm{~cm}$

For the track to be considered an isolated track, the measured calorimeter $E_{T}$ in a cone surrounding the track (minus the $E_{T}$ in calorimeter towers directly in line with the track), divided by the track $p_{T}$, must also be less than 0.1 . The cone is defined with a radius $\Delta R$ of 0.4, where $\Delta R$ is defined by

$$
\Delta R=\sqrt{(\Delta \eta)^{2}+(\Delta \phi)^{2}}
$$

with $\Delta \eta$ and $\Delta \phi$ being the distance in pseudorapidity and azimuth between a given calorimeter tower and the track.

\subsection{Jet Definition and Cuts}

A jet at CDF is a cluster of energy deposition in the calorimeters. This analysis searches for hadronic jets, which are jets resulting from quarks or gluons produced in a $p \bar{p}$ collision. Jets at CDF are reconstructed and defined using a jet clustering (JetClu) algorithm, which takes all calorimeter towers $i$ with transverse energy deposition $E_{T i}>1 \mathrm{GeV}$ and groups them together into jets. The first stage in the algorithm is to define a list of "seed towers" in order of decreasing $E_{T i}$. For each seed tower, "clusters" are built which contain all towers within a cone of radius $\Delta R$, defined using the $\Delta \eta$ and $\Delta \phi$ between the 
cluster centre and a given tower. In this analysis, a radius $\Delta R=0.7$ is used for the cone size of the jets: other CDF analyses use cone sizes of 0.4 or 1.0 [27].

The transverse energy and location of each cluster is calculated as a weighted average of the $E_{T i}, \phi_{i}$ and $\eta_{i}$ of each tower in the cluster:

$$
\begin{aligned}
& E_{T}=\sum E_{T i} \\
& \phi=\sum \frac{E_{T i} \phi_{i}}{E_{T}} \\
& \eta=\sum \frac{E_{T i} \eta_{i}}{E_{T}}
\end{aligned}
$$

with the sum going over all towers with $E_{T i}>1 \mathrm{GeV}$ within $R=0.7$ of the seed tower. This process is iterated, with a new list of towers around the new centre determined using the new $\eta$ and $\phi$. The jet's direction and $E_{T}$ are recalculated until the tower list is stable, with the seed tower's centre corresponding to the jet centre. If two jets overlap, they are merged if they share more than half their towers. If they overlap by less than $50 \%$, each of the shared towers are assigned to whichever jet is nearest. The final $E_{T}$ and direction information for the jet comes from the final set of towers [27].

\subsubsection{Jet Energy Scale and Corrections}

Jets in this analysis are corrected to the particle level, meaning that the corrected jet energy is the same as the initial energy of the initial quark or gluon which produced the jet. The jet must also be corrected to its true energy, removing any effects from variable calorimeter response or from energy deposition not related to the jet. Full jet

corrections thus require correcting the raw jet energies for the $\eta$-dependent response of different parts of the calorimeters, the possible presence of multiple $p \bar{p}$ interactions in an event, and for the overall jet energy scale (JES) which describes the response of the calorimeter to particles of a given energy [27].

The initial energy scale is set for the CEM and PEM by requiring that the $Z$ mass 
in $Z \rightarrow e \bar{e}$ events is consistent with the world average value. The hadronic calorimeters are initially calibrated using the results from a $50 \mathrm{GeV} / c$ charge pion test beam. As the scintillators and photomultiplier tubes in the calorimeters age, the energy scale decreases. This is corrected in the CEM by examining the measured $E / p$ of electrons with $E_{T}>$ $8 \mathrm{GeV}$. In the CHA and the WHA, a laser system, the muons from $J / \psi \rightarrow \mu^{+} \mu^{-}$decays, and the emissions from a $\mathrm{Cs}^{137}$ source are use to examine the change in energy scale. The two plug calorimeter systems are monitored with a laser system and a $\mathrm{Co}^{60}$ radioactive source. After the energy scale is calibrated, the calibration is checked by examining events in data. The $Z$ mass from $Z \rightarrow e \bar{e}$ candidate events are used for the CEM, PEM, and PHA, while the average energy for muons from $W^{-} \rightarrow \mu \bar{\nu}_{\mu}$ candidate events are used for CHA, WHA, and PHA calibration. The stability of these quantities over time is used to provide an uncertainty on the overall jet energy scale [27].

The response of the calorimeter is not uniform in $\eta$, due to differences in response for the five different calorimeter components, as well as the effects of the central crack at $\eta=0$ and the gaps at $|\eta| \approx 1.1$ between the central and plug calorimeters. The $\eta$ dependent corrections are determined by checking the $p_{T}$ ratios of the jets in dijet events, and applying $\eta$-dependent corrections until the jets are balanced. These corrections are determined using exclusive dijet events from Monte Carlo samples generated using the PYTHIA [31] and HERWIG [32] event generators, with a full detector simulation based on the GEANT [33] program. Comparison of the dijet balancing of these Monte Carlo samples with exclusive dijet events from data, after the application of the $\eta$-dependent corrections, yields the systematic uncertainties on these corrections [27].

The energy scale of the calorimeter is tuned to single particles in the calorimeter, not to the electromagnetic and hadronic showers which form jets in $p \bar{p}$ collisions. The same dijet Monte Carlo samples used for the $\eta$-dependent corrections are used to match simulated jets in the calorimeter with the generated partons. The $E_{T}$ distribution of the reconstructed jets is compared to the $p_{T}$ distribution of partons at the generator level to 
determine the calorimeter jet to parton corrections. The reconstructed jet energy from dijet events in data is compared to the total $p_{T}$ from tracks associated with the jets. The largest discrepancy in this comparison between the data and either the PYTHIA or a HERWIG Monte Carlo dijet sample is taken as a systematic uncertainty on the overall jet energy scale [27].

Multiple $p \bar{p}$ interactions may also contribute to the reconstructed jet energy if a secondary interaction results in energy deposition overlapping with a jet from a hard scattering event. This effect is measured by examining minimum bias events ${ }^{1}$ in data, and determining the average energy found in a randomly selected cone with $0.2<|\eta|<0.6$ and any value of $\phi$. The energy found in these random cones is parameterized as a function of the number of vertices found in the event, which is the best estimate of the number of additional $p \bar{p}$ interactions present. Jets can be corrected for the presence of multiple interactions by subtracting this amount of energy from each jet. An uncertainty on this correction comes primarily from the reconstruction efficiency and fake rate for the vertex finding algorithm [27].

In the remainder of this thesis, all jet transverse energies should be assumed to be fully corrected to the particle level. The total uncertainty on the scaling varies from $8 \%$ at low jet energies to $3 \%$ at higher energies, with the dominant sources of systematic uncertainty coming from the uncertainty on the test beam results which set the initial jet energy scale and on the corrections which take the reconstructed jet down to the parton level [27].

\subsubsection{Dijet Selection}

A previous monojet $+\not_{T}$ analysis at CDF looked for one jet accompanied by large $\not_{T}$, but also allowed a second jet with $E_{T}<30 \mathrm{GeV}$ [34]. Partly in order to keep the dataset

\footnotetext{
${ }^{1}$ The minimum bias data sample is triggered by coincident hits in both CLC counters. This means that its only requirement is that at least one $p \bar{p}$ interaction is present, and that the probability of energetic depositions in the central calorimeter is correspondingly low.
} 
in this analysis completely orthogonal to the monojet analysis, this analysis requires that the second-highest $E_{T}$ jet have at least $30 \mathrm{GeV}$ (which means $E_{T}>30$ for the leading jet.) The low- $E_{T}$ threshold for jets considered in this analysis is $15 \mathrm{GeV}$. Thus, to keep an exclusive dijet signature, any third jet found with $E_{T}>15 \mathrm{GeV}$ will cause the event to be rejected.

Badly measured jets in the event will lead to an imbalance in $E_{T}$ distribution, and thus "fake $\not_{T}$ ". For both jets passing the signal cuts, additional requirements are set to ensure the jets are well-measured:

- No jet can point to the "chimney" (1.05 rad $\left.<\phi<1.66 \mathrm{rad}, 0.5<\eta_{\text {det }}<1.0\right)$ or "crack" $\left(\left|\eta_{\text {det }}\right|<0.05\right)$ regions. Since the calorimeters do not cover these regions, any jets pointing to these areas of the detector are assumed to be badly measured.

- Between runs 217990 and 230545, problems with two of the PMTs in one of the WHA's towers (covering $-1.35<\eta<-1.15$ and $4.757 \mathrm{rad}<\phi<4.983 \mathrm{rad}$ ) caused random spikes in that tower's reported energy deposition. Since this produces a spurious high- $E_{T}$ jet object, and thus fake $E_{T}$, any event with a jet pointing to this "hot tower" during those runs is rejected.

- To further reduce the impact of fake $\not_{T}$ coming from other mismeasured jets, events where either jet is within $0.5 \mathrm{rad}$ in $\phi$ to the $E_{T}$ vector are rejected.

Removing events with badly measured jets significantly reduces background from QCD processes, leaving the production of $W$ or $Z$ bosons with additional jets as the main source of SM background for a dijet $+\not_{T}$ signature. The contribution from $W \rightarrow l \bar{\nu}$ and $Z \rightarrow l \bar{l}$ processes is reduced by applying an indirect lepton veto. This is accomplished by requiring both jets to have an EM fraction less than 0.9 , and by applying an isolated track veto (described in Section 4.1.) The EM fraction of a jet is simply $E_{E M}$, the energy deposited in the EM portion of its calorimeter towers, divided by the total jet energy $E$. Cuts are also placed on the jet charge fraction $(\mathrm{CHF})$, defined as 


$$
\mathrm{CHF}=\frac{\sum p_{T}}{E_{T}}
$$

where the sum runs over all tracks within $\Delta R=0.7$ of the jet axis. These cuts reject jet candidates which are not associated with tracks coming from $p \bar{p}$ collisions by requiring that energetic jets are associated with high- $p_{T}$ tracks in the COT. Without this cut, energy deposition unassociated with any $p \bar{p}$ collision products can be erroneously counted as a jet.

In the standard $\mathrm{CDF}$ jet object, the sum in the CHF calculation ranges over all jets, and the cut CHF $>0.1$ is imposed for all jets with $\left|\eta_{\text {det }}\right|<1.0$. However, this analysis uses an additional charge fraction variable (CHF2) which is calculated in the same way for each jet, but with the sum going over only those tracks which pass the following quality cuts:

- Five or more hits in three or more axial COT layers

- Five or more hits in two or more stereo COT layers

- Track $p_{T}>1.0 \mathrm{GeV} / c$

- $z_{0}$ within $10 \mathrm{~cm}$ of the event vertex

- $\left|z_{0}\right|<60 \mathrm{~cm}$

- $\left|d_{0}\right|<0.5 \mathrm{~cm}$

At least one jet must be central $\left(\left|\eta_{\text {det }}\right|<1.0\right)$, and have CHF2 $>0.05$, with at least two tracks meeting the above criteria contributing to the CHF2 calculation. This cut is to stop high- $p_{T}$ tracks which point in the general direction of a jet, but which are unassociated with the event vertex, from contributing to the CHF sum. 


\subsection{Clean-up Cuts}

Events in the data samples may have high $\not_{T}$ originating from non-collision backgrounds, such as detector noise, cosmic rays passing through the detector, or beam halo. Beam halo refers to particles produced from interactions between the particles in the beam and either residual gas in the beamline or other beam particles. Such particles travel mostly parallel to the beam, and may thus pass through several calorimeter towers. This results in unbalanced $E_{T}$ deposition, leading to $E_{T}$, unaccompanied by COT tracks [35].

The following clean-up cuts are placed on the event as a whole:

- At least five tracks must have been reconstructed in the inner detector, and they must be associated with a reconstructed vertex.

- For the reconstructed vertex, $\left|z_{v t x}\right|<60 \mathrm{~cm}$.

- The event EM fraction (EMF) must satisfy:

$$
\mathrm{EMF}=\frac{\sum E_{T}^{i} \cdot \frac{E_{E M}^{i}}{E_{T O T}^{i}}}{\sum E_{T}^{i}}>0.1
$$

where the sum is over all jets in the event with corrected $E_{T}>15 \mathrm{GeV}$.

In addition, the previously described CHF cuts act as quality cuts which filter out noncollision events.

Not all non-collision backgrounds will be removed by these cuts. A cosmic ray finding algorithm [36] can be used to remove events which have tracks consistent with a cosmic ray travelling through the detector, and the hadronic calorimeter timing information can identify energy deposition out of time with a collision. However, some genuine $p \bar{p}$ events can leave similar signatures. Instead of cutting out all events with these features, these non-collision backgrounds are estimated after examining the MET45 signal sample, and then added to the total background estimate from SM sources. The treatment of the remaining non-collision backgrounds which may survive these cuts is discussed in Subsection 5.4.3. 


\subsection{Kinematic Regions}

Two kinematic regions are defined for this analysis, in order to increase the range of possible new physics which can potentially be observed. The kinematic regions are defined using two kinematic quantities. The first is $\not_{T}$, as corrected to take into account the jet $E_{T}$ corrections described in Section 4.2. The second is $H_{T}$, defined as the scalar sum of the $E_{T}$ for the two jets, or

$$
H_{T}=E_{T}^{\mathrm{JET} 1}+E_{T}^{\mathrm{JET} 2}
$$

In the first kinematic region, called the low kinematic region because it has the lower $\not_{T}$ and $H_{T}$ cuts of the two regions examined, the $\not_{T}$ cut is $80 \mathrm{GeV}$. This is close to where the MET45 trigger is $99 \%$ efficient. The corresponding $H_{T}$ cut is $125 \mathrm{GeV}$. A lower $H_{T}$ cut would not make sense, as the $\#_{T}$ cut already forces the jets to have high $E_{T}$.

To further reduce QCD background, the high kinematic region increases the $Z_{T}$ cut to $100 \mathrm{GeV}$ and the $H_{T}$ cut to $225 \mathrm{GeV}$. Though these cuts are higher, they are still low enough that a data-driven estimate of the SM backgrounds can be made with an expected $10-15 \%$ uncertainty. The two kinematic regions are not exclusive: any event which passes the high kinematic cuts also passes the low kinematic cuts. However, the analyses are carried out independently. 


\section{Chapter 5}

\section{Standard Model Backgrounds}

\subsection{Overview}

The method used to determine the background to new physics signals in the dijet + $E_{T}$ signature is similar to that used in a previous signature-based analysis at CDF using a single high- $E_{T}$ jet plus $E_{T}$ as the signature [34]. It concentrates on determining background contributions using data samples containing events excluded from the final sample. All backgrounds are calculated without examining the events passing the final kinematic cuts, except for the background contribution due to non-collision events.

The largest backgrounds to possible new physics signals in this analysis are those from $W$ or $Z$ decay accompanied by jets. The contribution of these backgrounds is determined by using the high- $p_{T}$ electron and muon samples described in Section 3.2.

Another important, but harder to estimate, SM background comes from QCD multijet processes. This background is estimated using three-jet events in the MET45 data sample (described in Section 3.3) which fall outside the signal region due to the extra jet. This background has a larger relative uncertainty, but is much smaller than the electroweak contribution due to the stringent cuts on "fake" $E_{T}$ (as described in Section 4.2).

Other SM processes contributing to the background, namely $\gamma+$ jet production, $t \bar{t}$ 
production, and single top production, are estimated using Monte Carlo simulation normalized using previously measured Tevatron cross-sections or theoretical calculations. Finally, the residual contribution to the background from non-collision events is determined via examination of the events passing all signal cuts in the MET45 data sample and in the high- $p_{T}$ lepton samples.

\subsection{Electroweak Backgrounds}

The data-driven estimates of the electroweak backgrounds have three main steps:

- Identification of $Z$ and $W+$ dijet candidates in the high- $p_{T}$ electron and muon samples,

- Determination of the $Z \rightarrow l \bar{l}$ and $W^{-} \rightarrow l \bar{\nu}_{l}+$ dijet cross-sections for $l=e$ or $\mu$, and

- Determination of the $Z \rightarrow \nu \bar{\nu}+$ dijet cross-section using the $Z \rightarrow l \bar{l}$ and $W^{-} \rightarrow l \bar{\nu}_{l}$ + dijet cross-sections.

Strictly speaking, the $Z \rightarrow l \bar{l}$ and $W^{-} \rightarrow l \bar{\nu}_{l}+$ dijet cross-sections are the cross-sections for the production of $Z$ and $W+$ dijet events in $1.96 \mathrm{TeV} p \bar{p}$ collisions multiplied by the appropriate $W^{-} \rightarrow l \bar{\nu}_{l}, Z \rightarrow l \bar{l}$, or $Z \rightarrow \nu \bar{\nu}$ branching ratio, but they will be referred to as cross-sections in the remainder of this thesis.

\subsection{1 $Z$ and $W$ Identification in High- $p_{T}$ Muon Samples}

When searching for $Z$ and $W$ decay candidates within the high- $p_{T}$ muon sample, further requirements are set for muon identification. The track for a muon candidate must meet the following criteria [30]:

- Five or more hits must be present in three or more axial COT layers. 
- Five or more hits must be present in two or more stereo COT layers.

- Track $p_{T}>20.0 \mathrm{GeV} / c$.

- $\left|z_{0}\right|<60 \mathrm{~cm}$ (where $z_{0}$ is the track impact parameter along the $z$ axis of the detector).

- $\left|d_{0}\right|<0.02 \mathrm{~cm}$ for tracks with three or more hits in the silicon systems, or $0.2 \mathrm{~cm}$ for tracks with two hits or less.

- $E_{E M}$, the energy measured in electromagnetic calorimeter towers which the track intersects, is $<2 \mathrm{GeV}$ if muon momentum $P_{\mu}<100 \mathrm{GeV} / c$. For $P_{\mu} \geq 100 \mathrm{GeV} / c$, $E_{E M}<2+\left(\left(P_{\mu}-100\right) \cdot 0.0115\right) \mathrm{GeV}$.

- $E_{H A D}$, the energy measured in hadronic calorimeter towers which the track intersects, is $<6 \mathrm{GeV}$ if muon momentum $P_{\mu}<100 \mathrm{GeV} / c$. For $P_{\mu} \geq 100 \mathrm{GeV} / c$, $E_{H A D}<6+\left(\left(P_{\mu}-100\right) \cdot 0.0280\right) \mathrm{GeV}$.

- $\mu_{i s o}$, the calorimeter energy in a $\Delta R$ cone around the track after subtracting the energy in towers intersected by the track, must satisfy the condition $\mu_{i s o} / p_{T}<0.1$.

A cosmic ray tagging algorithm [36] is also run on the track associated with each muon candidate. If the algorithm can use the track to reconstruct an extended track going across two hemispheres of the detector, with $\chi^{2}<1000$ for the fit, the muon is rejected.

The sole muon candidate in a $W^{-} \rightarrow \mu \bar{\nu}_{\mu}$ candidate event must meet additional requirements. The muon must be either a "good CMUP" muon, which:

- Has an attached CMU stub with $|\Delta x<7| \mathrm{cm}$,

- Has an attached CMP stub with $|\Delta x<5| \mathrm{cm}$,

- Passes CMU fiducial cuts $d x<0 \mathrm{~cm}$ and $d z<0 \mathrm{~cm}$ 
- Passes CMP fiducial cuts $d x<0 \mathrm{~cm}$ and $d z<-3 \mathrm{~cm}$, and

- Does not include CMP muon candidates with stubs at $60^{\circ}<\phi<70^{\circ}$ for runs before 154449

or a "good CMX" muon, which:

- Does not include any CMX muon candidates found in runs before 150144,

- Does not include CMX muons with stubs in the west half of the detector with $\phi$ between $225^{\circ}$ and $240^{\circ}$ for runs between 190697 and 209541 , where that part of the CMX was not working properly,

- Does not include muons with stubs in the "miniskirt" or west "keystone" regions of the CMX,

- Passes CMX fiducial cuts $d x<0 \mathrm{~cm}$ and $d z<-3 \mathrm{~cm}$,

- Has an attached CMX stub with $|\Delta x<6| \mathrm{cm}$, and

- $\operatorname{Has} \rho_{C O T}=\left(\left(\left(\eta_{\mu} /\left|\eta_{\mu}\right|\right) \cdot 155.0\right)-z_{0} / \tan (\pi / 2)-\theta_{\mu}\right)>140 \mathrm{~cm}$.

For these requirements, the $z$ direction for a muon chamber is defined as the longitudinal direction of the drift wires, while the $x$ direction lies perpendicular to $z . \Delta x$ is defined as the distance in $x$ between the extrapolated position at the muon chamber of a muon candidate's COT track and the stub in the muon chamber. The fiducial distances $d x$ and $d z$ are defined as the distances in $x$ and $z$ between the extrapolated COT track and the outer edge of the muon chamber. The sign of $d x$ or $d z$ is defined so a negative value corresponds to a track extrapolated to lie within the muon chamber, meaning $d x<0 \mathrm{~cm}$ simply means extrapolation within the chamber in $x$, and $d z<-3 \mathrm{~cm}$ means the track is extrapolated to be at least $3 \mathrm{~cm}$ inside the chamber. All of these requirements must also be met by one or both of the muons in a $Z \rightarrow \mu \bar{\mu}$ candidate event. 
The second muon candidate in a $Z \rightarrow \mu \bar{\mu}$ candidate event need only pass a looser set of cuts. It only has to pass the initial track selection criteria, and is not required to be matched to any stubs in the muon detectors. It does, however, have to be a high-quality isolated track, with associated calorimeter deposits consistent with being a minimum ionizing particle. The invariant mass of the two muon candidates must be between 66 and $116 \mathrm{GeV} / c^{2}$. Finally, the three-dimensional opening angle between the two muon candidates, $\theta_{3 D}=\arccos \left(\left(P_{\mu}^{1} \cdot P_{\mu}^{2}\right) /\left(\mid\left(P_{\mu}^{1}|| P_{\mu}^{2} \mid\right)\right)\right)$, must be less than $3.70876 \mathrm{rad}$. This helps to reduce cosmic muon background which the cosmic tagging algorithm fails to reject.

For $W^{-} \rightarrow \mu \bar{\nu}_{\mu}$ there is one additional requirement on the single muon candidate which reduces the number of events from the decays of $\pi^{ \pm}$or $K^{ \pm}$mesons to a muon and a muon neutrino. The $\chi^{2}$ per degree of freedom for the muon candidate track, based only on COT information, must be less than 2.3 (or 2.7 for run numbers below 186598). This means that the muon track must be continuous in the COT, rather than representing a combination of a meson decaying within the COT and a muon produced from its decay. This requirement is absent for $Z \rightarrow \mu \bar{\mu}$ candidate events, since any competing sources of muon pairs should not pass the invariant mass and opening angle cuts. The event as a whole in $W^{-} \rightarrow \mu \bar{\nu}_{\mu}$ candidates must have exactly one isolated track, and have $\not_{T}>20 \mathrm{GeV}$. This is a corrected $\not_{T}$ : the raw $\not_{T}$ is first corrected to take into account the jet energy corrections applied at the analysis level, then corrected to take into account the presence of a muon by adding the $p_{T}$ of the muon candidate and subtracting the $E_{T}$ of any calorimeter tower it transversed in order to avoid double-counting.

\subsection{2 $Z$ and $W$ Identification in High- $p_{T}$ Electron Samples}

Identifying $Z$ and $W$ decay candidates within the high- $p_{T}$ electron sample works much like the process in the muon sample. Tracks matched to energy clusters in the electromagnetic calorimeter are considered "loose" electron candidates if [37]: 
- $E_{T}>20 \mathrm{GeV}$ for the EM cluster

- $p_{T}>10 \mathrm{GeV} / c$ for the track

- The track has five or more hits in three or more axial COT layers

- The track has five or more hits in two or more stereo COT layers

- $\left|z_{0}\right|<60 \mathrm{~cm}$

- $E_{H A D} / E_{E M}<0.055+0.00045 \cdot E$ for the towers associated with the track and electromagnetic cluster

- $\left|\eta_{\text {det }}\right|<1.0$ for the electromagnetic cluster (i.e. the electron is detected by the central calorimeter)

- The electron is not tagged by a conversion algorithm which searches for electrons or positrons produced from a $\gamma \rightarrow e \bar{e}$ process.

- The electron must be fiducial to the shower maximum proportional chambers in the CEM (the CES detectors).

The electron candidate in $W^{-} \rightarrow e \bar{\nu}_{e}$ candidate events, and at least one electron candidate in $Z \rightarrow e \bar{e}$, must also be a "tight" electron which meets these requirements:

- The ratio of the energy of the cluster and the momentum of the track must satisfy $0.5<E / p<2.0$

- $-3.0 \mathrm{~cm}<Q \cdot \Delta x<1.5 \mathrm{~cm}$, where $Q$ is the sign of the charge reconstructed for the track and $\Delta x$ is the distance in the $r \phi$ plane between the COT track and the best matching CES cluster. 
- $L_{s h r}$, a variable measuring the lateral development of the electron shower ${ }^{1}$ is below 0.2 .

- $\chi_{\text {strip }}^{2}$, a comparison of the CES shower profile to results from electron testbeam data, is less than 10 .

The sole electron candidate in $W^{-} \rightarrow e \bar{\nu}_{e}$ candidate events must also have:

- $E_{T}>25 \mathrm{GeV}$ for the cluster

- $E_{\text {iso }} / E_{T}<0.1$

In addition to the requirements on the electron candidate listed above, the $W^{-} \rightarrow e \bar{\nu}_{e}$ candidate events must have no more than one isolated track and have corrected $\not_{T}>$ $25 \mathrm{GeV}$. The isolated track in the event must be the one associated with the electron candidate. The corrections applied to the $\not_{T}$ in the electron case are different from those used in the muon case. The raw $\not_{T}$ is still corrected to take into account jet energy corrections. However, these corrections are not appropriate for the reconstructed jet associated with the electron candidate, since the electromagnetic calorimeter clusters have their energies correctly calibrated in the offline reconstruction. Thus, the calculations use the uncorrected $E_{T}$ for the jet corresponding to the electron candidate.

For $Z \rightarrow e \bar{e}$ candidate events, at least one electron candidate passing the "tight" cuts is required, with the second candidate passing the "loose" cuts. The invariant mass of the two electron candidates must be between 66 and $116 \mathrm{GeV} / c^{2}$. The events passing these cuts are called the "tight-loose" $Z \rightarrow e \bar{e}+$ jets sample. For comparison, a "tight-tight" sample, where the second electron candidate also passes the tight cuts, is used later when calculating QCD contamination in the electroweak backgrounds.

\footnotetext{
${ }^{1} L_{s h r}$ is defined as $L_{s h r}=0.14 \times \sum_{i} \frac{E_{i}^{a d j}-E_{i}^{\text {prob }}}{\sqrt{(0.14 \sqrt{E})^{2}+\left(\Delta E_{i}^{\text {prob }}\right)^{2}}}$, where $E_{i}^{a d j}$ is the measured energy in the cluster's $i$ th tower, $E_{i}^{\text {prob }}$ is the expected energy calculated from a shower profile parameterization from test-beam data (with $\Delta E_{i}^{\text {prob }}$ being the uncertainty), $E$ being the electromagnetic energy of the cluster, $0.14 \sqrt{E}$ coming from the CEM energy resolution, and the sum running over all towers in the cluster.
} 


\subsection{3 $Z$ and $W$ Inclusive Cross-sections}

With a sample of $W$ and $Z$ candidates selected in the high- $p_{T}$ lepton data, cross-sections for $W$ or $Z$ production can be measured. The first task is to make inclusive cross-section measurements for $W^{-} \rightarrow e \bar{\nu}_{e}, W^{-} \rightarrow \mu \bar{\nu}_{\mu}, Z \rightarrow e \bar{e}$, and $Z \rightarrow \mu \bar{\mu}$ production. The results are compared to previous inclusive measurements from CDF Run II analyses.

Acceptance for the $W$ and $Z$ candidates is determined through the examination of Monte Carlo samples for each process. These samples are generated using the PYTHIA [31] event generator, with a full detector simulation based on GEANT [33]. The only cuts applied are the $Z$ or $W$ selection cuts from Subsection 5.2.2. The clean-up cuts described in Section 4.3 are omitted. No cuts on jet multiplicity, individual jet characteristics, or $H_{T}$ are applied, and the only $E_{T}$ cut used is the $\not_{T}>25 \mathrm{GeV}$ cut used to select $W$ candidates. The requirement that an isolated track must have $z_{0}$ within $10 \mathrm{~cm}$ of the event vertex is also omitted for the inclusive cross-section measurement. This is because a $W$ or $Z$ candidate with no additional jets is only expected to have one or two tracks, respectively. Since at least six tracks are needed to find a good quality event vertex, a cut on the $\Delta z$ between track and event vertex would not make sense in this case. The acceptance is then simply the fraction of events in the sample which pass the $W$ or $Z$ selection criteria.

The calculations also take into account scale factors due to the efficiency of lepton reconstruction. These scale factors are standard CDF scale factors which are based on the standard electron and muon identification requirements used in this and other CDF analyses. These are discussed in more detail in Subsection 5.2.4.

Tables 5.1 and 5.2 show the inclusive cross-section results obtained using the acceptances from the PYTHIA Monte Carlo and the yields of $W$ or $Z$ candidate events in the data. The results can be compared both across the different data-taking periods (period 0 , periods 1 to 7 , and periods 8 to 12) as well as between the electron and muon channels for $W$ and $Z$ candidates. For the $W$ cross-sections, the cross-sections are in 


\begin{tabular}{|l|r|r|r|}
\hline$W^{-} \rightarrow e \bar{\nu}_{e}$ & Run Period 0 & Run Periods 1-7 & Run Periods 8-12 \\
\hline Acceptance & $0.1775 \pm 0.0001$ & $0.1775 \pm 0.0001$ & $0.1752 \pm 0.0001$ \\
Raw Data & 190910 & 363819 & 498892 \\
Background & $(4.49 \pm 0.97) \%$ & $(4.8 \pm 1.1) \%$ & $(5.9 \pm 1.5) \%$ \\
Efficiency SF & $0.9406 \pm 0.0086$ & $0.9725 \pm 0.0084$ & $0.940 \pm 0.011$ \\
Luminosity & $375 \mathrm{pb}^{-1}$ & $724 \mathrm{pb}^{-1}$ & $1047 \mathrm{pb}^{-1}$ \\
\hline$\sigma(W)[\mathrm{pb}]$ & $2912 \pm 40$ & $2772 \pm 41$ & $2724 \pm 55$ \\
\hline \hline \hline$W^{-} \rightarrow \mu \bar{\nu}_{\mu}$ & Run Period 0 & Run Periods 1-7 & Run Periods 8-12 \\
\hline Acceptance & $0.1832 \pm 0.0002$ & $0.1808 \pm 0.0002$ & $0.1823 \pm 0.0001$ \\
Raw Data & 183745 & 340118 & 494975 \\
Background & $(12.52 \pm 0.70) \%$ & $(12.53 \pm 0.74) \%$ & $(13.05 \pm 0.92) \%$ \\
Efficiency SF & $0.8565 \pm 0.0058$ & $0.8852 \pm 0.0042$ & $0.866 \pm 0.013$ \\
Luminosity & $373 \mathrm{pb}^{-1}$ & $675 \mathrm{pb}^{-1}$ & $967 \mathrm{pb}^{-1}$ \\
\hline$\sigma(W)[\mathrm{pb}]$ & $2746 \pm 30$ & $2754 \pm 28$ & $2819 \pm 52$ \\
\hline \hline
\end{tabular}

Table 5.1: Input parameters and results for the $W$ inclusive cross section measurements made to verify the event selection and efficiency scale factors obtained from the joint physics group. The measurements are made for three separate run periods encompassing the full data sample.

good agreement across the different run periods. Only in run period 0 do the $W^{-} \rightarrow e \bar{\nu}_{e}$ and $W^{-} \rightarrow \mu \bar{\nu}_{\mu}$ results not agree within two standard deviations. For the $Z$ cases, the results for each channel agree within two standard deviations over the different run periods, and the three results in the $Z \rightarrow e \bar{e}$ channel are within two standard deviations of the $Z \rightarrow \mu \bar{\mu}$ result in the same run period. 


\begin{tabular}{|l|r|r|r|}
\hline$Z \rightarrow e \bar{e}$ & Run Period 0 & Run Periods 1-7 & Run Periods 8-12 \\
\hline Acceptance & $0.0876 \pm 0.0002$ & $0.0876 \pm 0.0002$ & $0.0882 \pm 0.0001$ \\
Raw Data & 8819 & 16853 & 23760 \\
Background & $(0.6 \pm 0.1) \%$ & $(0.7 \pm 0.1) \%$ & $(2.3 \pm 0.1) \%$ \\
Efficiency SF & $0.9919 \pm 0.0092$ & $1.0237 \pm 0.0098$ & $1.0106 \pm 0.0076$ \\
Luminosity & $375 \mathrm{pb}^{-1}$ & $724 \mathrm{pb}^{-1}$ & $1047 \mathrm{pb}^{-1}$ \\
\hline$\sigma(Z)[\mathrm{pb}]$ & $269 \pm 4$ & $258 \pm 3$ & $249 \pm 3$ \\
\hline \hline$Z \rightarrow \mu \bar{\mu}$ & Run Period 0 & Run Periods 1-7 & Run Periods 8-12 \\
\hline Acceptance & $0.1436 \pm 0.0003$ & $0.1431 \pm 0.0002$ & $0.1442 \pm 0.0002$ \\
Raw Data & 12748 & 23503 & 34378 \\
Background & $\mathrm{negl}$. & $\mathrm{negl}$. & $(0.15 \pm 0.03) \%$ \\
Efficiency SF & $0.9088 \pm 0.0074$ & $0.9385 \pm 0.0073$ & $0.942 \pm 0.017$ \\
Luminosity & $373 \mathrm{pb}^{-1}$ & $675 \mathrm{pb}^{-1}$ & $967 \mathrm{pb}^{-1}$ \\
\hline$\sigma(Z)[\mathrm{pb}]$ & $262 \pm 3$ & $259 \pm 3$ & $261 \pm 5$ \\
\hline \hline
\end{tabular}

Table 5.2: Input parameters and results for the $Z$ inclusive cross section measurements made to verify the event selection and efficiency scale factors obtained from the joint physics group. The measurements are made for three separate run periods encompassing the full data sample. 


\subsection{4 $Z$ and $W+$ Dijet Cross-sections}

The most relevant cross-sections for the background calculations are those for $Z$ and $W$ + dijet events passing the low and high kinematic region cuts. To get a sample of such events from the high- $p_{T}$ data samples or from the Monte Carlo samples, all signal cuts are applied to events, but with the electrons or muons from $W$ or $Z$ decay removed from the event. This means that the cuts are modified to treat the events as though the electrons or muons were invisible particles. Removing the charged leptons makes $W^{-} \rightarrow l \bar{\nu}_{l}$ or $Z \rightarrow l \bar{l}$ events look like $Z \rightarrow \nu \bar{\nu}$ events, which are the largest source of background for the dijet $+\not_{T}$ signature.

The new $E_{T}$ is recalculated as though the electrons or muons were not present. The isolated track cut is modified to require no additional isolated tracks beyond the

one required to make a $W^{-} \rightarrow l \bar{\nu}_{l}$ candidate event or the two required for a $Z \rightarrow l \bar{l}$ candidate. The jets matched to the electron candidates are also eliminated from the jet list. All other cuts are left unmodified, including all cleanup cuts and the $\Delta z$ requirement for isolated tracks omitted for the inclusive cross-section measurement.

\section{Acceptance Calculation and Corrections}

The acceptance for $W$ and $Z+$ dijet candidate events is measured using Monte Carlo samples generated using ALPGEN [38], using GEANT once again for the full detector simulation. Samples for $W^{-} \rightarrow e \bar{\nu}_{e}, W^{-} \rightarrow \mu \bar{\nu}_{\mu}, Z \rightarrow e \bar{e}$, and $Z \rightarrow \mu \bar{\mu}$ are generated with one, two, three, or four additional partons in order to create samples with one, two, three, or four additional jets. These samples are combined to represent the range of $W$ and $Z+$ jets actually expected in data. A simple combination of the samples weighted according to the relative cross-section for $W$ and $Z+1,2,3$, or 4 jets would not suffice, however. This is because the CDF simulation adds final state radiation subsequent to particle generation, meaning, for example, that a two parton event with an additional jet from final state radiation can be indistinguishable from an event with three partons 
produced at the generator level. A procedure referred to as MLM matching [39] removes duplicate events from the ALPGEN samples by matching the partons produced at the generator level to the jets produced. Events where any of the partons cannot be linked to a particular jet are rejected, as they are considered to be duplicates of events produced from a sample generated with one less parton but with an additional jet from final state radiation. After removal of duplicate events, the different ALPGEN samples are normalized by assigning a weight taken from the theoretical cross-section of each sample divided by the total number of events remaining in the sample.

Additionally, the ALPGEN samples used simulated beam conditions during runs up to run period 8 , while the data samples extended to run period 12 . The average instantaneous luminosity increases for later run periods. This could affect $W$ and $Z$ acceptance due to the higher number of mean interactions per collision at the higher luminosities seen in later run periods, which could change the efficiencies of selection criteria such as the isolation cut on lepton identification. It is therefore necessary to check if the acceptance is luminosity (and thus run period) dependent. This is done by re-weighting the Monte Carlo events as a function of the number of reconstructed vertices in the event in order to reproduce the distribution observed over all run periods 0 through 12 . The acceptance from these re-weighted events is less than $1 \%$ different from those obtained from the non-weighted Monte Carlo events, a discrepancy which falls within the existing statistical uncertainties.

There is a possible second order effect from the ALPGEN $Z$ samples being generated with a different $M_{Z}$ range than is used for $Z$ selection. While the ALPGEN samples used a $Z$ mass window of 75 to $105 \mathrm{GeV} / \mathrm{c}^{2}$, the $Z$ selection applied in this analysis is 66 to $116 \mathrm{GeV} / \mathrm{c}^{2}$. Since both the numerator and the denominator of the acceptance calculations count events selected with the same mass windows, this is expected to be a second order effect. To check this, PYTHIA $Z+1$ jet samples generated with a wider $Z$ mass range were used. The acceptances for $Z$ events were compared for both the 66 to 
$116 \mathrm{GeV} / \mathrm{c}^{2}$ and the 75 to $105 \mathrm{GeV} / \mathrm{c}^{2}$ mass windows. The observed difference is used to obtain a scale factor of 1.001 which is applied to the acceptances measured from the ALPGEN samples.

In the acceptance calculation for $W / Z+$ dijet events, the denominator is the combined number of events from simulation which pass the full dijet $+\not_{T}$ criteria for the low or high kinematic cuts after the removal of the leptons. The numerator is the subset of these events which also pass the $W$ or $Z$ lepton selection criteria.

\section{QCD Contamination in Electroweak Backgrounds}

The QCD background for the $W^{-} \rightarrow e \bar{\nu}_{e}+$ dijet candidate sample is determined using a technique described in Reference [40], which has also been used in the previous $\not_{T}+$ single jet studies [34]. In this method, the electron isolation distribution is compared for signal and QCD-enriched background samples of electron candidates from data events. The requirement for two electrons in $Z$ candidate events ensures very low QCD contamination, especially if both are required to satisfy tight requirements. Thus, electrons from $Z \rightarrow e \bar{e}$ events where both electron candidates satisfying all the tight electron selection criteria, with the exception of the isolation requirements, can be used to model the isolation of $W^{-} \rightarrow e \bar{\nu}_{e}$ electrons. A QCD-enriched data sample can be constructed from electrons in $W^{-} \rightarrow e \bar{\nu}_{e}$ candidates for which some combination of one or more of the electron identification cuts are required to fail. This sample combines electron candidates which fail the $E_{H A D} / E_{E M}$ cut and the CES $\chi^{2}$ cut; electron candidates which fail the $E_{H A D} / E_{E M}$ and CES $\delta x$ and $\delta z$ cuts; electron candidates which fail the CES $\delta x, \delta z$, and $\chi^{2}$ cuts; and electron candidates which fail the $E_{H A D} / E_{E M}, \mathrm{CES} \delta x, \mathrm{CES} \delta z$, and CES $\chi^{2}$ cuts.

The isolation distribution of both sets of electron candidates is then compared to the isolation distribution of the electron candidates in the $W^{-} \rightarrow e \bar{\nu}_{e}+$ dijet candidates, with the isolation distribution templates from the $Z \rightarrow e \bar{e}$ electron candidates and 
from the QCD-enriched samples adjusted to agree with the isolation distribution from $W^{-} \rightarrow e \bar{\nu}_{e}$ candidates. The normalization from the QCD-enriched template is used to estimate the QCD contamination in the $W^{-} \rightarrow e \bar{\nu}_{e}+$ dijet candidates, which is then removed from the $W^{-} \rightarrow e \bar{\nu}_{e}+$ dijet background estimate. The statistical uncertainty on this QCD contribution is calculated by fluctuating each bin of the QCD-enriched and the $Z \rightarrow e \bar{e}$ isolation distribution templates according to a Gaussian distribution. This is done $\mathcal{O}(10000)$ times in a series of pseudo-experiments. The resulting statistical uncertainty comes directly from the width of the resulting distribution of QCD background predictions. An additional systematic uncertainty comes from checking the QCD contamination when using a fake template made from only one of the sub-samples in the QCD-enriched sample - the sub-samples with reversed $E_{H A D} / E_{E M}$ and CES $\chi^{2}$ cuts; reversed $E_{H A D} / E_{E M}$, CES $\delta x$, and $\delta z$ cuts; and reversed CES $\delta x, \delta z$, and $\chi^{2}$ cuts. This allows the uncertainty to take into account the possibility that one of the cuts used biases the QCD contamination prediction, thus making the fake electron isolation template fail to reflect the actual fake electron distribution. The differences between the QCD prediction for the combined QCD-enriched sample and the QCD prediction using those three sub-samples is combined in quadrature and used as a systematic uncertainty.

The QCD contamination in the $W^{-} \rightarrow \mu \bar{\nu}_{\mu}+$ dijets sample is extracted from the distribution of $E_{T}$ versus muon isolation in the data events. This contribution is smaller in the $W^{-} \rightarrow \mu \bar{\nu}_{\mu}$ case than it is in the $W^{-} \rightarrow e \bar{\nu}_{e}$ case, since a QCD jet is more likely to be misidentified as an electron. In this method, the $W^{-} \rightarrow \mu \bar{\nu}_{\mu}+$ dijet requirements are applied to the muon sample, but with the muon isolation requirement and $F_{T}$ cuts left out. Three sideband regions to the $W^{-} \rightarrow \mu \bar{\nu}_{\mu}+$ dijet candidate sample are defined: region $\mathrm{A}$ has low isolation and $\mathscr{E}_{T}$, region $\mathrm{B}$ has low isolation but high $\not_{T}$, and region $\mathrm{C}$ has high isolation and high $\mathbb{E}_{T}$. The signal region, where the single muon candidate passes the isolation cut and the event has high $E_{T}$, can be called region D. Assuming that $E_{T}$ is uncorrelated with the isolation of a jet which is mistaken for a muon, the 
formula

$$
\frac{N_{A}}{N_{B}}=\frac{N_{D}}{N_{C}}
$$

(with $N_{i}$ the number of events in region $i$ ) can be used to find $N_{D}$, the QCD contamination. This method has been used in other CDF analyses for QCD contamination in $W^{-} \rightarrow e \bar{\nu}_{e}$ backgrounds as well, but it cannot be used for $W^{-} \rightarrow e \bar{\nu}_{e}$ in this case due to its lower precision and the fact that $\not_{T}$ and electron isolation are correlated for QCD multijet events containing jets identified as electron candidates [40].

The QCD contamination in $Z \rightarrow l \bar{l}$ events is small, and in fact turns out to be negligible for $Z \rightarrow \mu \bar{\mu}+$ dijets. It is estimated using the number of like-sign events which otherwise pass the $Z \rightarrow l \bar{l}+$ dijet requirements in the data. This estimate is corrected using the ALPGEN Monte Carlo for the number of $Z \rightarrow l \bar{l}$ events expected to be reconstructed as like-sign events.

\section{Scale Factors}

As with the inclusive cross-section measurements, standard CDF scale factors are applied in this analysis to account for trigger efficiencies and observed differences in the lepton selection cut efficiencies between data and Monte Carlo. These scale factors are based on the lepton identification cuts, which are the standard ones for CDF analyses.

In later run periods, a standard prescription exists which corrects for the effects of changes in instantaneous luminosity and dynamic pre-scaling of the muon triggers [30]. The applied scale factors change slightly as a function of the leading jet $E_{T}$ cut for the muon cross-sections. This is because the scale factors for muons found in the CMUP and CMX are independent, and the relative fractions of CMUP and CMX muons vary with leading jet $E_{T}$.

The inclusive and dijet cross-section measurements also use different scale factors. This is partly because the inclusive Monte Carlo samples for the early data (run period 
0 ) was produced with version 5 of the CDF analysis software, while all other Monte Carlo samples (including those used over the entire run range for the dijet cross-section measurements) were produced with version 6 . Also, the scale factors for the inclusive measurements must include a data versus Monte Carlo efficiency correction for the selection of a reconstructed vertex within $60 \mathrm{~cm}$ of the interaction point. For the dijet cross-section, this is not necessary, as the dijet cuts include the requirement that a vertex be reconstructed with $z_{0}<60 \mathrm{~cm}$.

\section{Results}

The cross-sections for $W^{-} \rightarrow l \bar{\nu}_{l}$ and $Z \rightarrow l \bar{l}+$ dijet events are in Tables 5.3 and 5.4, using the full set of signal cuts for both kinematic regions and with the charged leptons from boson decay removed. The uncertainties are mostly statistical, but systematic uncertainties such as those on QCD contamination are also included. The results are not necessarily expected to be the same across all run periods here, because the cross-section is dependent on jets reconstructed within the detector above a given $E_{T}$ threshold. At the higher luminosities of the later run periods, additional minimum bias interactions will contribute more underlying energy within each jet candidate in the event, lowering the effective jet $E_{T}$ threshold. Luminosity weighting is used to combine the results from the different run periods into a single cross-section for the entire electron and muon samples.

The correction factors applied to the $W / Z+$ dijet cross-sections results are applied in order to get unified $Z \rightarrow l \bar{l}$ and $W^{-} \rightarrow l \bar{\nu}_{l}+$ dijet cross-section measurements, which will be combined in the next section into a $Z \rightarrow \nu \bar{\nu}+$ dijet cross-section. These correction factors account for differences in event topology between $Z \rightarrow \nu \bar{\nu}$ decay and $W$ and $Z$ decays involving the much more massive charged leptons. For example, when eliminating jets associated with leptons on $Z \rightarrow e \bar{e}$ or $W^{-} \rightarrow e \bar{\nu}_{e}$ events, some fraction of the available phase space for extra jets in the event is removed which is available for $Z \rightarrow \nu \bar{\nu}$ events. Electroweak radiation from charged leptons can also produce extra 
jets in an event. The correction factor which takes this into account is calculated by comparing the fractions of $Z \rightarrow \nu \bar{\nu}$ and $Z \rightarrow l \bar{l}$ Monte Carlo events which satisfy the full dijet signal cuts after the charged leptons are removed from the $Z \rightarrow l \bar{l}$ events. The correction factors are simply the ratio of the two fractions. For $W^{-} \rightarrow l \bar{\nu}_{l}$, the correction factors are calculated assuming that the effects which make them necessary scale linearly with the number of charged leptons: in other words, the correction factors for $W^{-} \rightarrow l \bar{\nu}_{l}$ are one half those for $Z \rightarrow l \bar{l}$. The corrections are on the order of $5 \%$ to $10 \%$. The uncertainties include a statistical component from the size of the Monte Carlo samples used, and a systematic component obtained from the discrepancy in correction factors calculated from ALPGEN and PYTHIA samples.

Table 5.5 gives the combined corrected $W / Z+$ dijet cross-sections for both muon and electron channels. Since the agreement between the electron and muon results is good, the electron and muon measurements are combined to give $Z \rightarrow l \bar{l}$ and $W^{-} \rightarrow l \bar{\nu}_{l}$ results. The uncertainties on the $Z \rightarrow l \bar{l}$ and $W^{-} \rightarrow l \bar{\nu}_{l}+$ dijet results come from the uncorrelated uncertainties on the electron and muon results.

\subsection{5 $Z \rightarrow \nu \bar{\nu}+$ Dijets Background}

As the largest single background to the dijet $+\not_{T}$ signature, it is important to estimate the $Z \rightarrow \nu \bar{\nu}+$ dijets background as precisely as possible. The most obvious way to determine this background is to use the relation between the cross-section for $Z \rightarrow \nu \bar{\nu}$ production and the average $Z \rightarrow l \bar{l}$ cross-section:

$$
\sigma(Z \rightarrow \nu \bar{\nu}+\text { dijets })=5.942 \times \sigma(Z \rightarrow l \bar{l}+\text { dijets }) .
$$

The $W^{-} \rightarrow l \bar{\nu}_{l}+$ dijets cross-section measurement has a lower relative uncertainty than the $Z \rightarrow l \bar{l}+$ dijets cross-section due to its higher statistics. The analysis can take advantage of this by using the $W^{-} \rightarrow l \bar{\nu}_{l}$ result to find a $Z \rightarrow \nu \bar{\nu}+$ dijets cross-section, using 


\begin{tabular}{|l|r|r|}
\hline$W^{-} \rightarrow e \bar{\nu}_{e}$ & $H_{T}>125, E_{T}>80$ & $H_{T}>225, \not_{T}>100$ \\
\hline Acceptance & $0.2465 \pm 0.0035$ & $0.309 \pm 0.012$ \\
Raw Data & 508 & 65 \\
Background & $30.0 \pm 5.8 \%$ & $28.9 \pm 4.7 \%$ \\
Efficiency SF & $0.9606 \pm 0.0096$ & $0.9606 \pm 0.0096$ \\
\hline$\sigma(W)[\mathrm{pb}]$ & $0.700 \pm 0.074$ & $0.072 \pm 0.014$ \\
\hline Cor. Factor & $0.972 \pm 0.017$ & $1.005 \pm 0.043$ \\
Cor. $\sigma(W)[\mathrm{pb}]$ & $0.680 \pm 0.073$ & $0.073 \pm 0.014$ \\
\hline \hline$W^{-} \rightarrow \mu \bar{\nu}_{\mu}$ & $H_{T}>125, E_{T}>80$ & $H_{T}>225, \not_{T}>100$ \\
\hline Acceptance & $0.2591 \pm 0.0040$ & $0.323 \pm 0.013$ \\
Raw Data & 363 & 40 \\
Background & $25.5 \pm 1.7 \%$ & $33.0 \pm 4.3 \%$ \\
Efficiency SF & $0.867 \pm 0.013$ & $0.880 \pm 0.015$ \\
\hline$\sigma(W)[\mathrm{pb}]$ & $0.596 \pm 0.046$ & $0.047 \pm 0.012$ \\
\hline Cor. Factor & $1.071 \pm 0.016$ & $0.993 \pm 0.042$ \\
Cor. $\sigma(W)[\mathrm{pb}]$ & $0.638 \pm 0.050$ & $0.047 \pm 0.012$ \\
\hline \hline
\end{tabular}

Table 5.3: Input parameters and results for the $W$ plus dijet cross section measurements where the jet and $\mathbb{E}_{T}$ requirements are the same as those used to select the dijet plus missing $E_{T}$ event sample. The integrated luminosities of the electron and muon samples are $2148 \mathrm{pb}^{-1}$ and $2017 \mathrm{pb}^{-1}$, respectively. 


\begin{tabular}{|l|r|r|}
\hline$Z \rightarrow e \bar{e}$ & $H_{T}>125, \not_{T}>80$ & $H_{T}>225, \not_{T}>100$ \\
\hline Acceptance & $0.1546 \pm 0.0049$ & $0.2028 \pm 0.0080$ \\
Raw Data & 23 & 4 \\
Background & $24.3 \pm 8.5 \%$ & $30 \pm 24 \%$ \\
Efficiency SF & $1.0176 \pm 0.0071$ & $1.0176 \pm 0.0071$ \\
\hline$\sigma(Z)[\mathrm{pb}]$ & $0.052 \pm 0.016$ & $0.006 \pm 0.005$ \\
\hline Cor. Factor & $0.962 \pm 0.022$ & $0.933 \pm 0.038$ \\
Cor. $\sigma(Z)[\mathrm{pb}]$ & $0.050 \pm 0.015$ & $0.006 \pm 0.005$ \\
\hline \hline$Z \rightarrow \mu \bar{\mu}$ & $H_{T}>125, \not_{T}>80$ & $H_{T}>225, \not_{T}>100$ \\
\hline Acceptance & $0.2517 \pm 0.0078$ & $0.332 \pm 0.012$ \\
Raw Data & 42 & 7 \\
Background & $4.9 \pm 1.0 \%$ & $2.6 \pm 0.6 \%$ \\
Efficiency SF & $0.947 \pm 0.034$ & $0.918 \pm 0.063$ \\
\hline$\sigma(Z)[$ pb] & $0.083 \pm 0.014$ & $0.011 \pm 0.004$ \\
\hline Cor. Factor & $1.117 \pm 0.015$ & $1.114 \pm 0.041$ \\
Cor. $\sigma(Z)[\mathrm{pb}]$ & $0.093 \pm 0.016$ & $0.012 \pm 0.005$ \\
\hline
\end{tabular}

Table 5.4: Input parameters and results for the $Z$ plus jets cross section measurements where the jet and $E_{T}$ requirements are the same as those used to select the dijet plus missing $E_{T}$ event sample. The integrated luminosities of the electron and muon samples are $2148 \mathrm{pb}^{-1}$ and $2017 \mathrm{pb}^{-1}$, respectively. 


\begin{tabular}{|l|c|c|}
\hline Cross Section $(\mathrm{pb})$ & $H_{T}>125, \not_{T}>80$ & $H_{T}>225, \not_{T}>100$ \\
\hline \hline$\sigma\left(W^{-} \rightarrow e \bar{\nu}_{e}+\right.$ jets $)$ & $0.68 \pm 0.07$ & $0.073 \pm 0.014$ \\
\hline$\sigma\left(W^{-} \rightarrow \mu \bar{\nu}_{\mu}+\right.$ jets $)$ & $0.64 \pm 0.05$ & $0.047 \pm 0.012$ \\
\hline$\sigma\left(W^{-} \rightarrow l \bar{\nu}_{l}+\right.$ jets $)$ & $0.65 \pm 0.04$ & $0.057 \pm 0.009$ \\
\hline$\sigma(Z \rightarrow e \bar{e}+$ jets $)$ & $0.05 \pm 0.02$ & $0.006 \pm 0.005$ \\
\hline$\sigma(Z \rightarrow \mu \bar{\mu}+$ jets $)$ & $0.09 \pm 0.02$ & $0.012 \pm 0.005$ \\
\hline$\sigma(Z \rightarrow l \bar{l}+$ jets $)$ & $0.07 \pm 0.01$ & $0.009 \pm 0.003$ \\
\hline
\end{tabular}

Table 5.5: Combined $W / Z+$ jet cross section measurements used to estimate backgrounds in the dijet plus missing $E_{T}$.

$$
\sigma(Z \rightarrow \nu \bar{\nu}+\text { dijets })=5.942 \times \sigma\left(W^{-} \rightarrow l \bar{\nu}_{l}+\text { dijets }\right) \times R
$$

where

$$
R=\frac{\sigma\left(W^{-} \rightarrow l \bar{\nu}_{l}+\text { dijets }\right)}{\sigma(Z \rightarrow l \bar{l}+\text { dijets })}
$$

and the factor 5.942 comes from the ratio of the $Z$ decay widths for $Z \rightarrow l \bar{l}$ and $Z \rightarrow \nu \bar{\nu}$ multiplied by a factor of 3 to take into account the three types of neutrinos.

A program called MCFM, for "Monte Carlo for FeMtobarn processes" [41], is used to calculate $R$ as follows. $W$ and $Z$ plus two parton cross-sections are calculated at nextto-leading order (NLO) with MCFM. The generated cross-sections are used to produce two sets of cross-section weighted " $E_{T}$ " distributions for both the $W$ and $Z$ production processes. In this calculation, " $E_{T}$ " is actually the $E_{T}$ of the $W$ or $Z$, which is equivalent to the $E_{T}$ recalculated to ignore the contributions from charged leptons in the $Z \rightarrow l \bar{l}$ or $W^{-} \rightarrow l \bar{\nu}_{l}$ cases. One " $E_{T}$ " distribution is generated for each signal region, with the low kinematic region distribution generated with an $H_{T}$ cut of $125 \mathrm{GeV}$, and the high kinematic region distribution generated with an $H_{T}$ cut of $225 \mathrm{GeV}$. The cross-section 


\begin{tabular}{|l|c|c|}
\hline Candidate Sample & $H_{T}>125, E_{T}>80$ & $H_{T}>225, \not_{T}>100$ \\
\hline \hline Calculated $R$ & 8.68 & 8.18 \\
\hline Monte Carlo Statistics & \pm 0.028 & \pm 0.026 \\
\hline Uncertainty from PDFs & \pm 0.043 & \pm 0.040 \\
\hline Uncertainty from Jet Energy Scale & \pm 0.14 & \pm 0.13 \\
\hline Uncertainty from Renormalization Scale & \pm 0.06 & \pm 0.13 \\
\hline \hline Total Uncertainty & \pm 0.15 & \pm 0.19 \\
\hline
\end{tabular}

Table 5.6: Values for $R$ obtained from MCFM theoretical calculations.

for each process is then integrated above the $\not_{T}$ cut used to define the appropriate signal region (either $80 \mathrm{GeV}$ or $100 \mathrm{GeV}$.) The $W$ boson result is divided by the $Z$ boson result, thus obtaining a value for $R$. The results for $R$ appear in Table 5.6.

Table 5.6 also contains statistical and systematic uncertainties on $R$. The systematic uncertainty from the choice of parton distribution function (PDF) is calculated by redoing the MCFM calculation with different PDF choices. Similarly, the renormalization scale uncertainty is calculated by redoing the calculations with different choices for the renormalization scale. The final source of uncertainty comes from the possibility that the generator-level $E_{T}$ distribution is not exactly the same as the $\not_{T}$ distribution in the data. This is dealt with by checking the variation of $R$ over a $\not_{T}$ range consistent with the jet energy scale uncertainties in data and Monte Carlo.

Using the $R$ values calculated from MCFM, two separate predictions of the $Z \rightarrow \nu \bar{\nu}$ contribution to the background can be made: one from the $Z \rightarrow l \bar{l}+$ dijets results, and one from the $W^{-} \rightarrow l \bar{\nu}_{l}+$ dijets results. Table 5.7 shows these results, as well as the estimate for the $Z \rightarrow \nu \bar{\nu}+$ dijet background combining the two predictions. 


\begin{tabular}{|l|c|c|}
\hline$Z \rightarrow \nu \nu$ Prediction & $H_{T}>125, \not_{T}>80$ & $H_{T}>225, \not_{T}>100$ \\
\hline \hline Sample Luminosity & $2039 \mathrm{pb}^{-1}$ & $2039 \mathrm{pb}^{-1}$ \\
\hline From $\sigma(Z \rightarrow l \bar{l}+$ jets $)$ & $836 \pm 129$ & $111 \pm 41$ \\
\hline From $\sigma\left(W^{-} \rightarrow l \bar{\nu}_{l}+\right.$ jets $)$ & $899 \pm 59$ & $84 \pm 13$ \\
\hline Combined Estimate & $888 \pm 54$ & $86 \pm 13$ \\
\hline
\end{tabular}

Table 5.7: Predicted numbers of $Z \rightarrow \nu \bar{\nu}$ events.

\subsection{6 $W^{-} \rightarrow l \bar{\nu}_{l}+$ Dijets Background}

The $W^{-} \rightarrow l \bar{\nu}_{l}+$ dijet events are also a significant contribution to the backgrounds. The measured $W^{-} \rightarrow l \bar{\nu}_{l}+$ dijet cross-sections can be used to estimate these contributions.

To find the predicted contribution from $W^{-} \rightarrow \mu \bar{\nu}_{\mu}$ events, the fraction of events in which the muon is not reconstructed, or otherwise fails to cause the event to be rejected by the isolated track veto and other cuts, is determined from the Monte Carlo sample. The measured $W^{-} \rightarrow \mu \bar{\nu}_{\mu}+$ dijets cross-section before corrections is then multiplied by this fraction of events where the muon is "lost", the integrated luminosity of the high- $p_{T}$ muon sample, and a trigger efficiency correction which accounts for the efficiency of the MET45 trigger at a $\not_{T}$ of $80 \mathrm{GeV}$ (or $100 \mathrm{GeV}$ for the high kinematic region.) ${ }^{2}$

The $W^{-} \rightarrow e \bar{\nu}_{e}$ background is calculated in the same way. Due to the wider coverage of the EM calorimeter compared to the muon detectors, electrons are less likely to be lost. The electron is lost in $20.8 \pm 0.3 \%$ of $W^{-} \rightarrow e \bar{\nu}_{e}$ events in the low kinematic region, compared to $33 \pm 1 \%$ of $W^{-} \rightarrow \mu \bar{\nu}_{\mu}$ events where the muon is lost. Thus, their overall contribution to the background is less.

In the $W^{-} \rightarrow \tau \bar{\nu}_{\tau}+$ dijet case, the $\tau$ may be "lost" if its decay does not lead to an isolated track or another feature which identifies it as a charged lepton, or it may be reconstructed as a jet. To estimate this background, the fraction of $W^{-} \rightarrow \tau \bar{\nu}_{\tau}$

\footnotetext{
${ }^{2}$ See Section 3.3.1.
} 
+ jets events in MC that pass the dijet $+\not_{T}$ cuts are compared to the fraction of $W^{-} \rightarrow \mu \bar{\nu}_{\mu}+$ jets events passing the same selections. This gives an estimate for the "loss" of $W^{-} \rightarrow \tau \bar{\nu}_{\tau}+$ jets events with respect to the measured $W^{-} \rightarrow \mu \bar{\nu}_{\mu}+\operatorname{dijet}$ cross-section. In the low kinematic region, $54.6 \pm 0.8 \%$ of the $W^{-} \rightarrow \tau \bar{\nu}_{\tau}$ events have a lost $\tau$.

\subsection{7 $Z \rightarrow l \bar{l}+$ Dijets Background}

The $Z \rightarrow l \bar{l}$ backgrounds where $l$ is a charged lepton are also determined. This turns out to be a small contribution, since both charged leptons must be lost for such events to contribute. The prediction is made in the same way as the $W^{-} \rightarrow l \bar{\nu}_{l}$ case, but using the measured $Z \rightarrow l \bar{l}+$ dijets cross-sections instead.

Table 5.10 at the end of this chapter shows the background estimates for all $W$ and $Z$ processes, together with the contributions from the other SM processes discussed in Sections 5.3 and 5.4.

\subsubsection{Cross-checks with ALPGEN Monte Carlo}

This technique for determining the electroweak backgrounds is intended to have minimal dependence on the details of the Monte Carlo modeling. Nevertheless, since ALPGEN Monte Carlo samples are used to determine the acceptance for $W$ and $Z+$ dijet events, some of the kinematic distributions which come from the Monte Carlo samples need to be cross-checked with the data.

The histograms in Figure 5.1 show comparisons of the electron $\eta$ and $p_{T}$ distributions for electrons in $W^{-} \rightarrow e \bar{\nu}_{e}+$ dijet data and ALPGEN Monte Carlo events, before and after the low kinematic region $E_{T}$ and $H_{T}$ cuts. Figure 5.2 shows the same distributions for the first and the second electron in $Z \rightarrow e \bar{e}+$ dijet data and Monte Carlo, before $E_{T}$ or $H_{T}$ cuts are applied. In both cases, the agreement is good. Figures 5.3 and 5.4 show the same plots for the muons in $W^{-} \rightarrow \mu \bar{\nu}_{\mu}+$ dijet and $Z \rightarrow \mu \bar{\mu}+$ dijet events, 

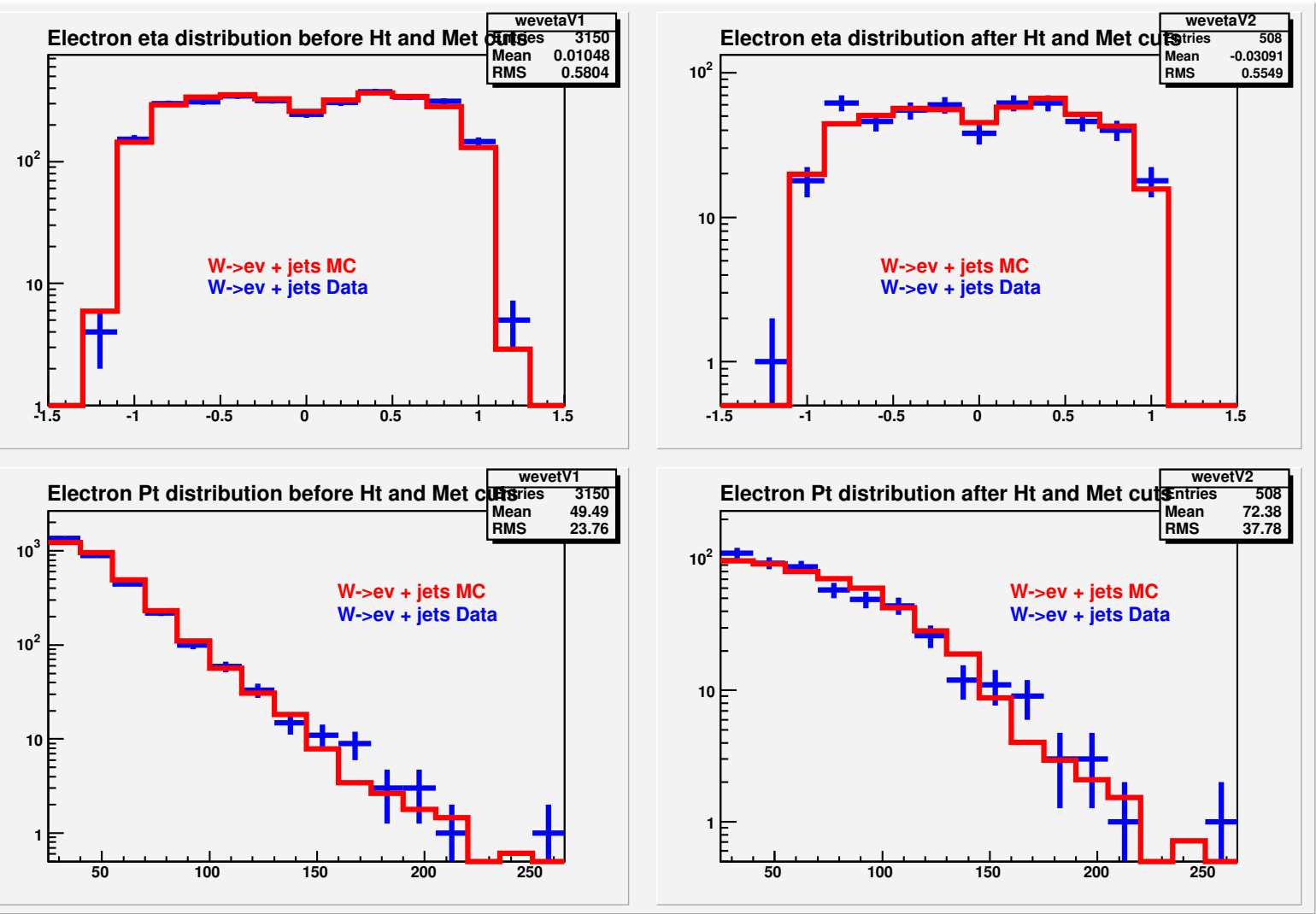

Figure 5.1: Comparison of electron kinematic distributions between ALPGEN Monte Carlo and data for $W^{-} \rightarrow e \bar{\nu}_{e}+$ jets candidate sample.

respectively. The muon kinematic distributions also show good agreement between data and ALPGEN.

The background measurement technique in this analysis is designed to be insensitive to the Monte Carlo modeling for jets, and thus to the $E_{T}$, jet $E_{T}$, and $H_{T}$ distributions in the ALPGEN samples. This is because the acceptance measured from Monte Carlo depends only on the probability for $W$ or $Z$ identification from lepton reconstruction. Comparisons of the kinematic distributions for the jets can still be made, however. Since the QCD contamination in the electron samples is significant, and a good model for the resulting kinematic distributions for jets is not available, only the muon samples are examined. Figure 5.5 compares the $E_{T}$ of the first and second jets in $W^{-} \rightarrow \mu \bar{\nu}_{\mu}+$ dijet events in ALPGEN and in data. For $Z \rightarrow \mu \bar{\mu}+$ dijet events in ALPGEN and data, 

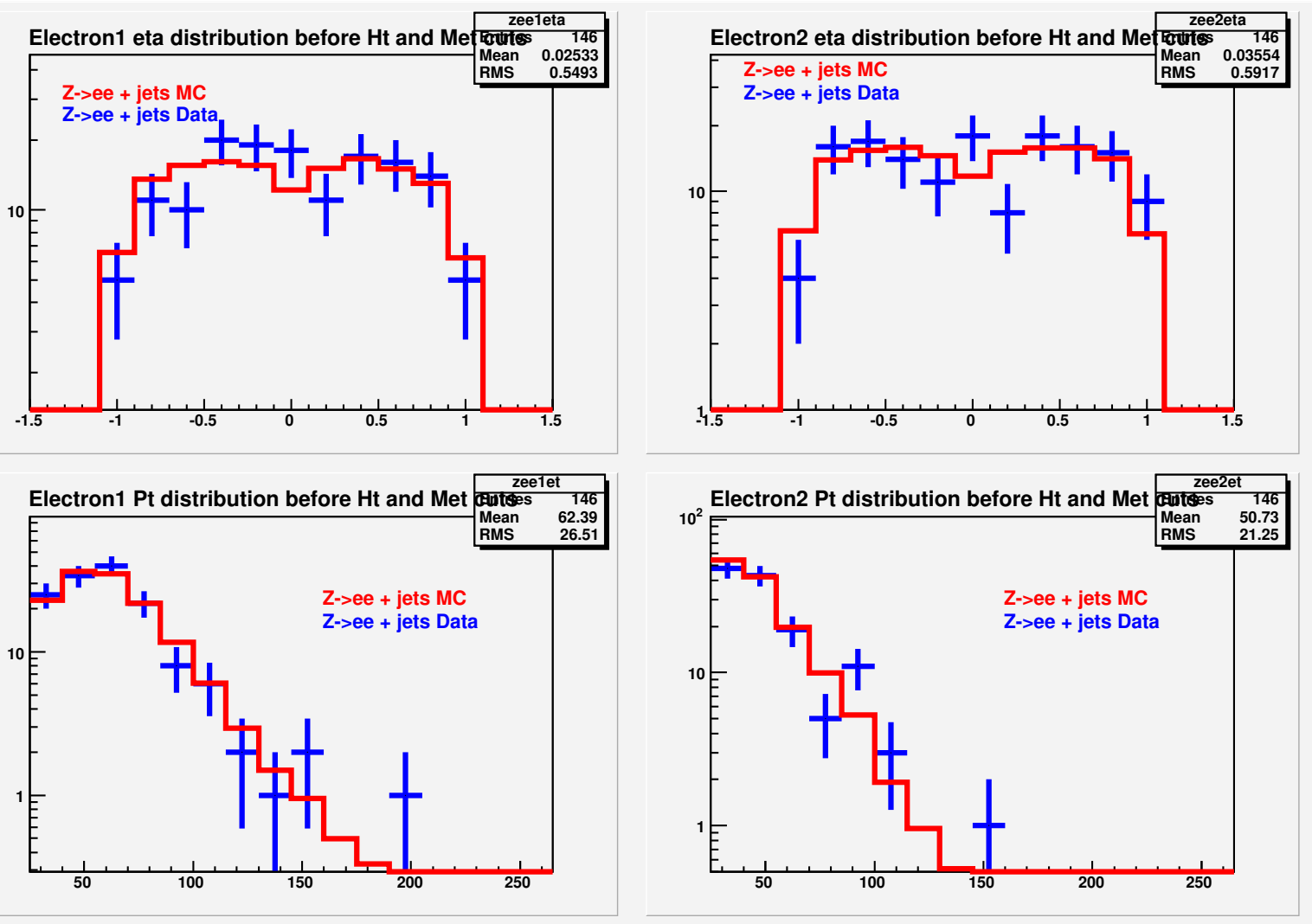

Figure 5.2: Comparison of electron kinematic distributions between ALPGEN Monte Carlo and data for $Z \rightarrow e \bar{e}+$ jets candidate sample.

Figure 5.6 compares the $E_{T}$ and $\eta$ distributions for the first and second jets, and Figure 5.7 shows the $\Delta \phi$ between $E_{T}$ and either jet, as well as the $E_{T}$ and $H_{T}$ distributions. In all cases, the ALPGEN distributions agree with the data.

A final set of comparisons appears in Figure 5.8. The reconstructed $Z$ mass distribution and transverse $W$ mass distribution for $Z \rightarrow \mu \bar{\mu}$ and $W^{-} \rightarrow \mu \bar{\nu}_{\mu}+$ dijet events from ALPGEN show good agreement with the data.

\subsection{QCD Backgrounds}

While the jet cuts of Section 4.2 and kinematic cuts of Section 4.4 greatly reduce the background contribution from QCD multijet events, some events may still pass the cuts 

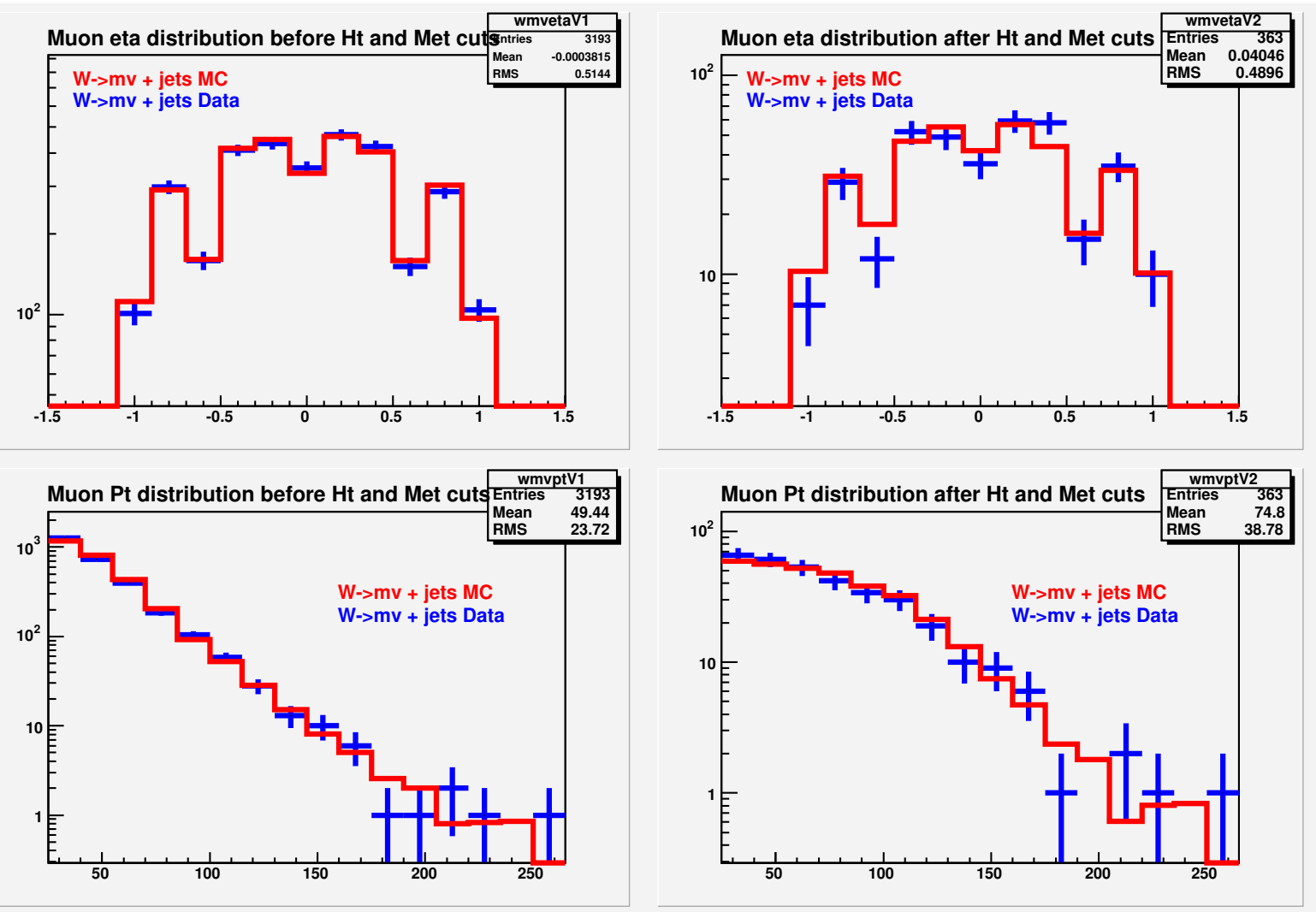

Figure 5.3: Comparison of muon kinematic distributions between ALPGEN Monte Carlo and data for $W^{-} \rightarrow \mu \bar{\nu}_{\mu}+$ jets candidate sample.

and enter the signal samples. Since these cuts require exactly two jets which are more than $0.5 \mathrm{rad}$ from the direction of the $\mathbb{E}_{T}$ in $\phi$, QCD background in this analysis must come from events where an additional jet's energy is mismeasured badly enough to fall below the $E_{T}>15 \mathrm{GeV}$ threshold for the jet to be counted.

The dominant QCD background is events where one jet is mismeasured so it appears to fall below the $15 \mathrm{GeV}$ threshold. Such a jet would be predominantly responsible for the observed $E_{T}$. In these events, $\phi\left(E_{T}\right)$ will be close to the $\phi$ of the "lost" jet. This analysis uses the data to estimate how many such events exist. A smaller contribution comes from events where the $\mathbb{F}_{T}$ is due to more than one jet's mismeasurement. In this case, the exclusive dijet requirement still requires that at least one jet is lost. The contribution from these events is dealt with via simulation. 

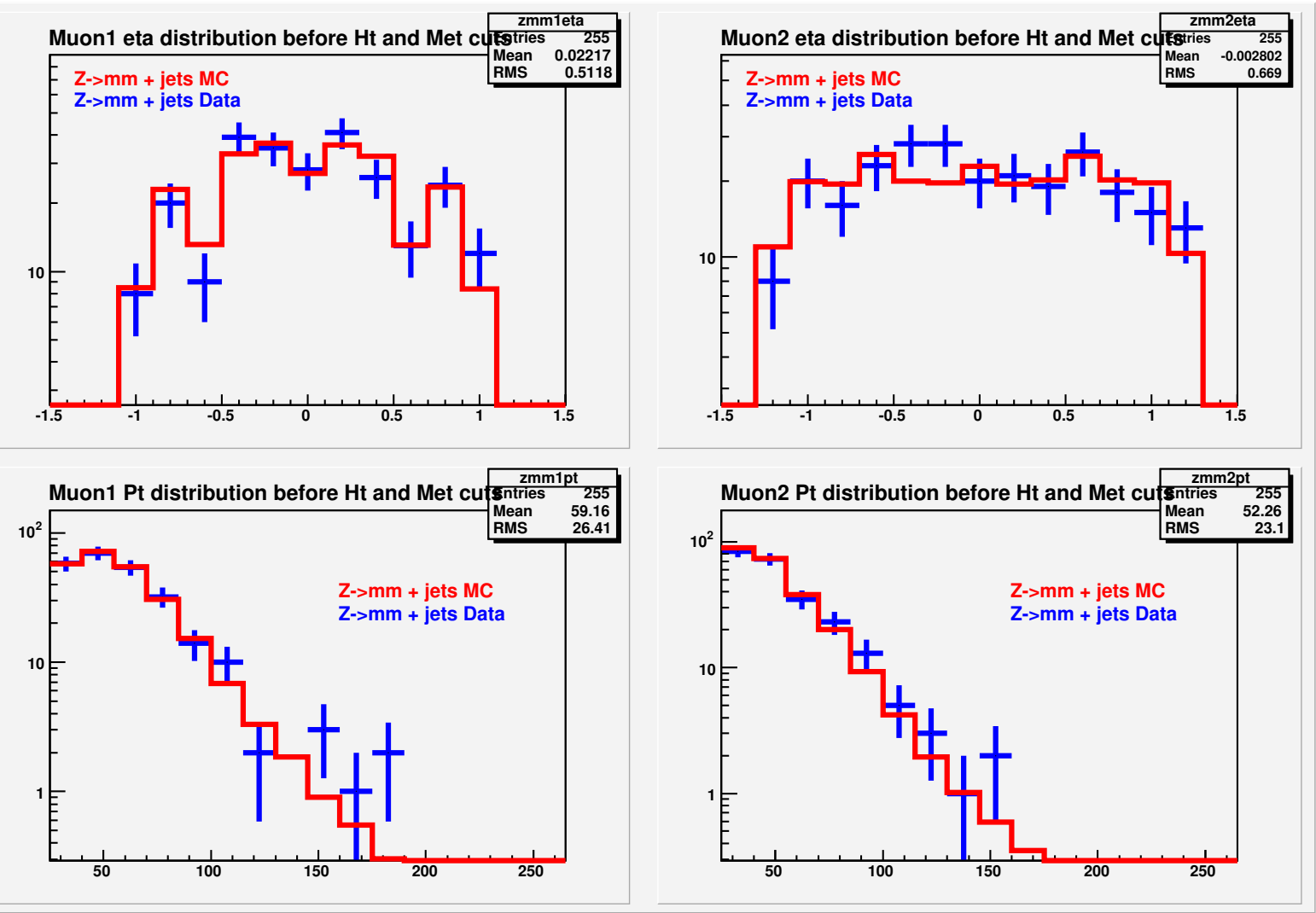

Figure 5.4: Comparison of muon kinematic distributions between ALPGEN Monte Carlo and data for $Z \rightarrow \mu \bar{\mu}+$ jets candidate sample.

The first category of QCD background events is measured by taking the same MET45 data used to search for signal, and selecting the events where $\Delta \phi=\mid \phi\left(E_{T}\right)-\phi($ Jet3) $\mid<$ $0.3 \mathrm{rad}$. All other cuts for the low and high kinematic region are used, except for the cut requiring exactly two jets: instead, three or more jets are required. The $E_{T}$ of the third leading jet in the event is examined, then extrapolated to the $E_{T}<15 \mathrm{GeV}$ region to determine how many events have a third jet which is lost entirely due to mismeasurement. Corrections must, however, be made due to electroweak backgrounds found in these events.

The electroweak corrections affect the data normalization. In the QCD events, about $98 \%$ of events have $\Delta \phi<0.3 \mathrm{rad}$ for the third jet. On the other hand, events in electroweak background Monte Carlo samples have a basically flat $\Delta \phi$ distribution between 

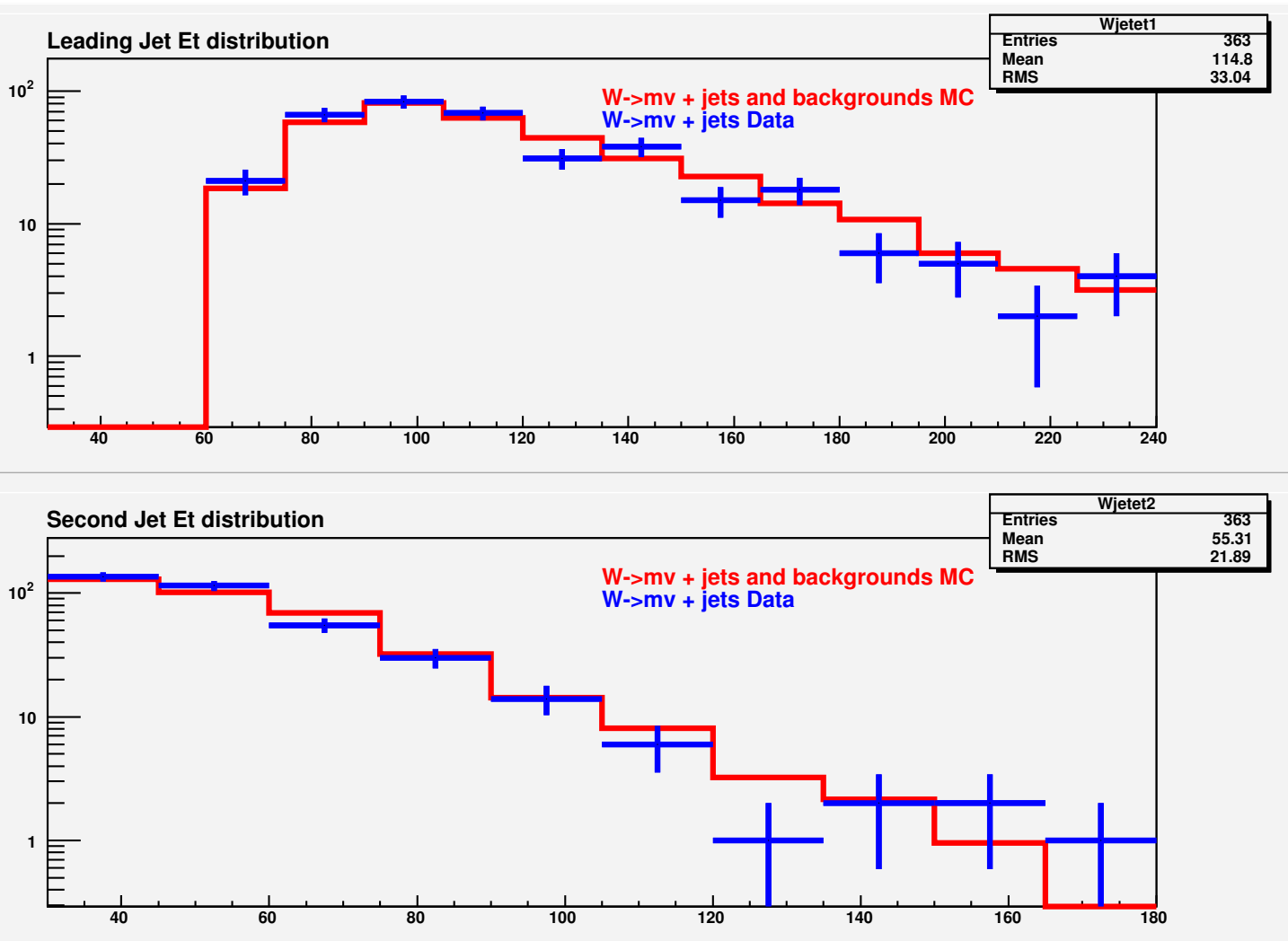

Figure 5.5: Comparison of jet kinematic distributions between ALPGEN Monte Carlo and data for $W^{-} \rightarrow \mu \bar{\nu}_{\mu}+$ jets candidate sample.

$0.0 \mathrm{rad}$ and $0.5 \mathrm{rad}$. This can be seen in Figure 5.9 for $W^{-} \rightarrow \tau \bar{\nu}_{\tau}$ events and Figure 5.10 for $W^{-} \rightarrow \mu \bar{\nu}_{\mu}$ events. The region between $0.3 \mathrm{rad}$ and $0.5 \mathrm{rad}$ can therefore be used as the electroweak normalization region. Since the distributions are flat, the number of events with $0.3 \mathrm{rad}<\Delta \phi<0.5 \mathrm{rad}$ (a range of $0.2 \mathrm{rad}$ ) can be multiplied by 1.5 to get an estimate for the number of electroweak events over the $0.0 \mathrm{rad}<\Delta \phi<0.3 \mathrm{rad}$ range.

The distribution of the third jet's $E_{T}$ for electroweak backgrounds must also be checked. In the simulation, these distributions turn out to be the same for $0.0 \mathrm{rad}<$ $\Delta \phi<0.3 \mathrm{rad}$ as they are for $0.3 \mathrm{rad}<\Delta \phi<0.5 \mathrm{rad}$. Thus, the distributions for the data in the $0.3 \mathrm{rad}$ to $0.5 \mathrm{rad}$ region are used. From the data normalization and the third jet $E_{T}$ distribution, the electroweak background in the region of interest can be subtracted in the region of interest, $0.0 \mathrm{rad}<\Delta \phi<0.3 \mathrm{rad}$, by renormalizing. 

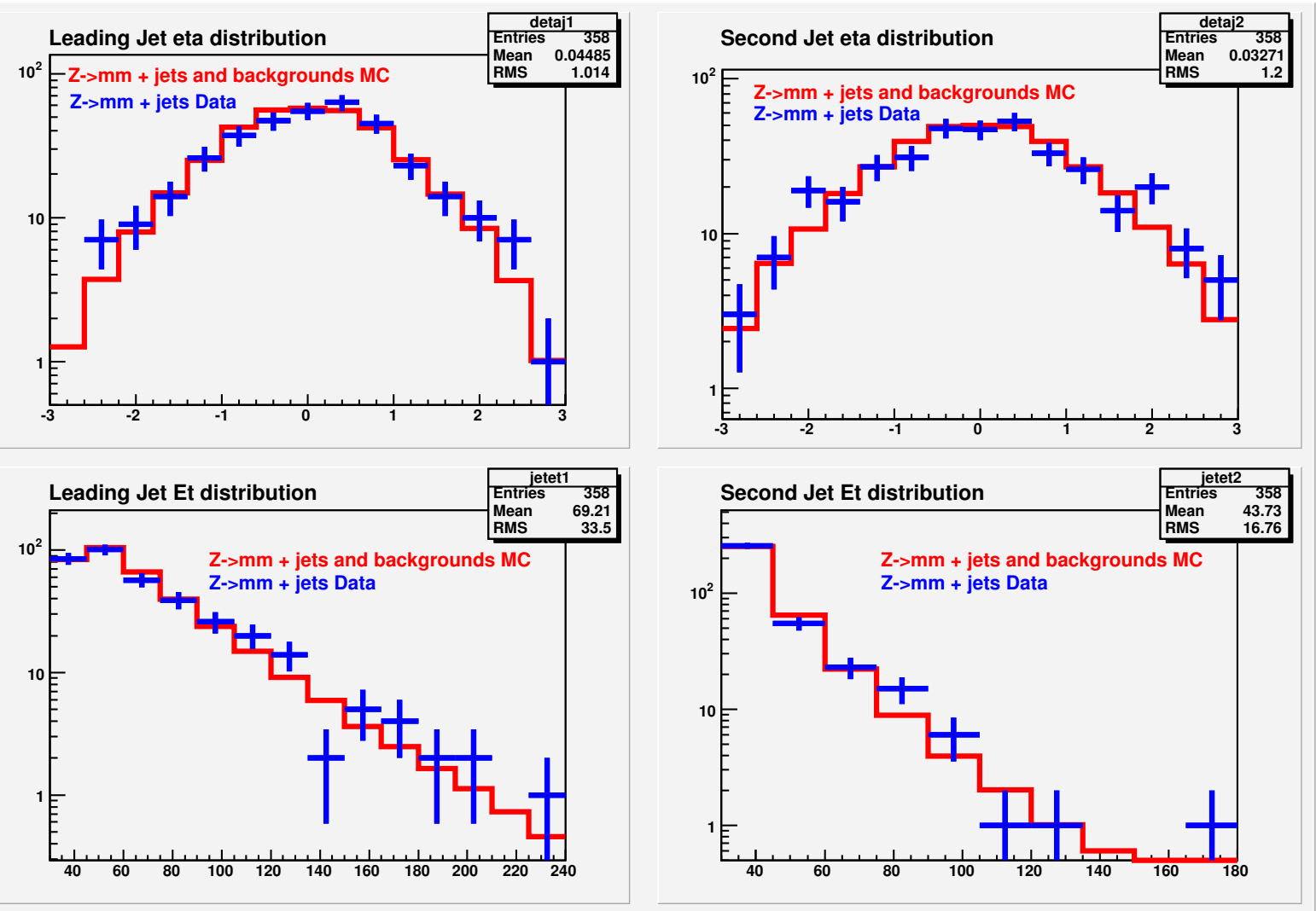

Figure 5.6: Comparison of jet kinematic distributions (I) between ALPGEN Monte Carlo and data for $Z \rightarrow \mu \bar{\mu}+$ jets candidate sample.

An additional correction is made which takes into account photon + jet events. Simulation with PYTHIA shows that the third leading jet in these events has similar $\Delta \phi$ distributions between 0.0 and $0.5 \mathrm{rad}$. This simulation is therefore used to estimate the normalization correction for $\Delta \phi$ between 0.0 and $0.3 \mathrm{rad}$.

Figure 5.11 shows the distribution of the $E_{T}$ for the third jet, after correction for the electroweak and photon contributions, in events passing all the signal cuts for the low kinematic region except for the exclusive dijet cut. The fit produced from this distribution is extrapolated to $0 \mathrm{GeV}$, and then integrated up to $15 \mathrm{GeV}$. The resulting integral is 39 events. This is the estimated number of events where one lost jet is responsible for the $\not_{T}$. Simulation predicts that these represent $78 \%$ of the QCD background events. The estimate is therefore adjusted to 49 events, to account for the predicted $22 \%$ contribution 

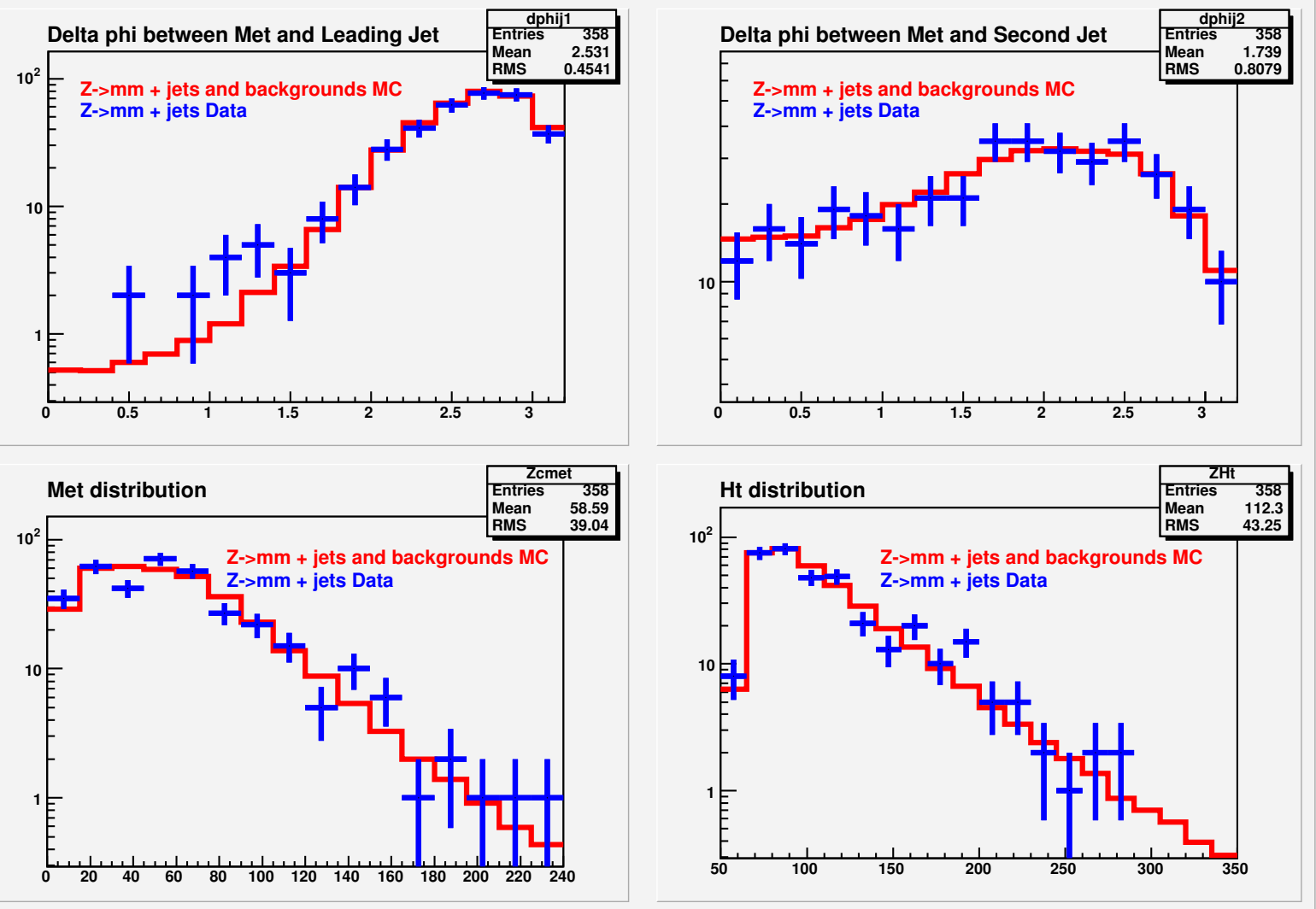

Figure 5.7: Comparison of jet kinematic distributions (II) between ALPGEN Monte Carlo and data for $Z \rightarrow \mu \bar{\mu}+$ jets candidate sample.

from events where at least two mismeasured jets account for the $E_{T}$. The systematic uncertainty of 30 events comes from:

- a $50 \%$ uncertainty in the relative contribution from events with two or more mismeasured jets (making it $22 \pm 11 \%$ ),

- a $10 \%$ relative variation on the scale factor used to normalize the electroweak contribution,

- and a $50 \%$ relative uncertainty on the correction from photon + jet events.

The uncertainty on the scale factor used to normalize the electroweak contribution comes from comparisons of data-subtracted distributions to the general shape found in the simulated distribution shown in Figure 5.12. If the scale factor is changed by more than 

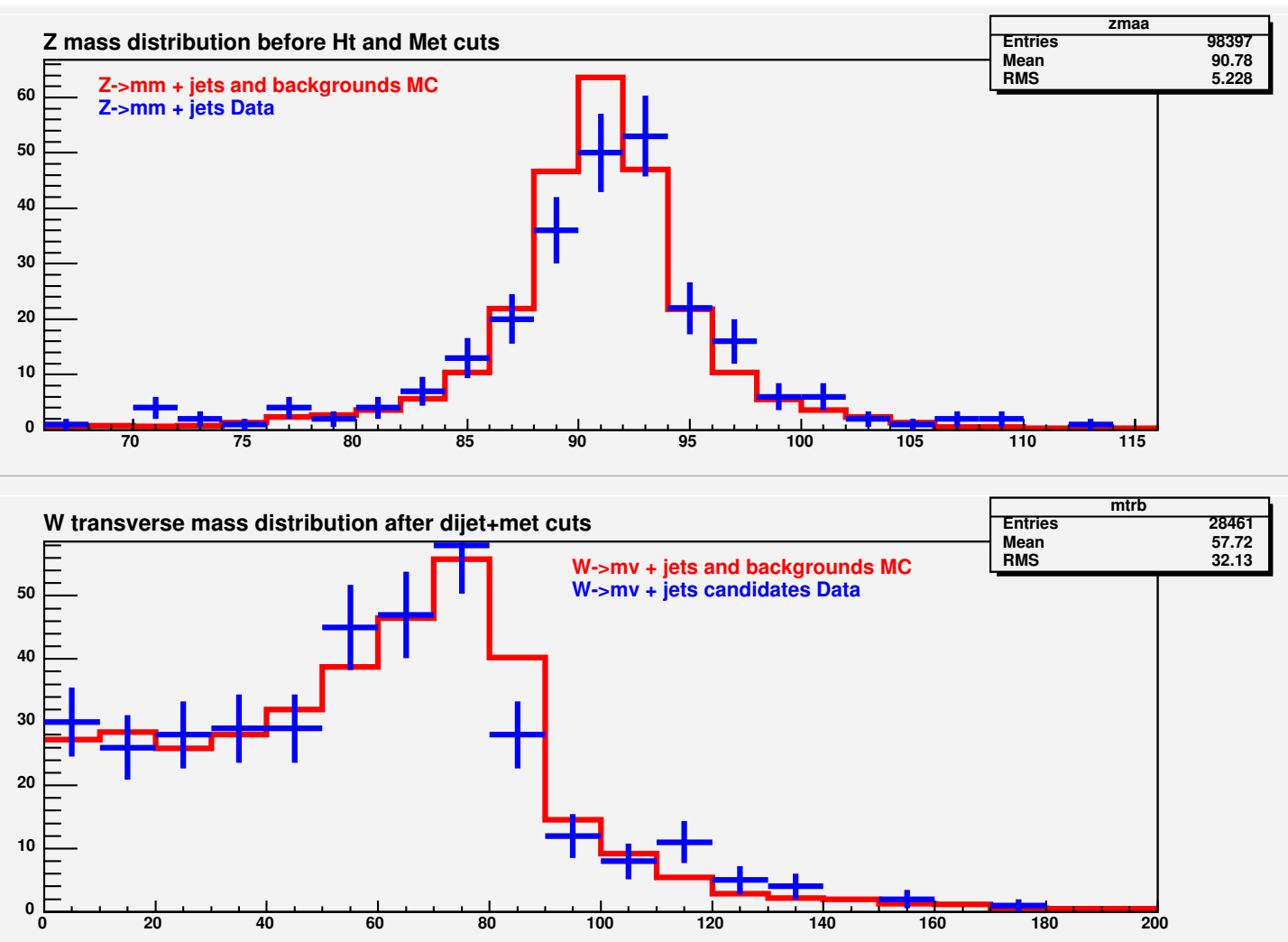

Figure 5.8: Comparisons of the $Z$ invariant mass (top) and $W$ transverse mass (bottom) distributions between ALPGEN Monte Carlo and data for $Z \rightarrow \mu \bar{\mu}+$ jets and $W^{-} \rightarrow \mu \bar{\nu}_{\mu}+$ jets candidate samples.

$10 \%$, the data distributions become inconsistent with a smoothly falling exponential distribution.

For the high kinematic region, the assumptions made for the electroweak corrections are checked to ensure that they are still valid in the high kinematic region, and the estimate is performed in a similar manner. The result is $9 \pm 9$ events. The relative uncertainty for the high kinematic region is higher, because of the lower statistics in both the data samples and the Monte Carlo samples used.

A cross-check of this result was performed using simulated data. A PYTHIA Monte Carlo sample of QCD dijet events was analyzed, and the signal cuts were applied. Assuming the PYTHIA leading order calculation for the QCD dijet cross-section, the two Monte 


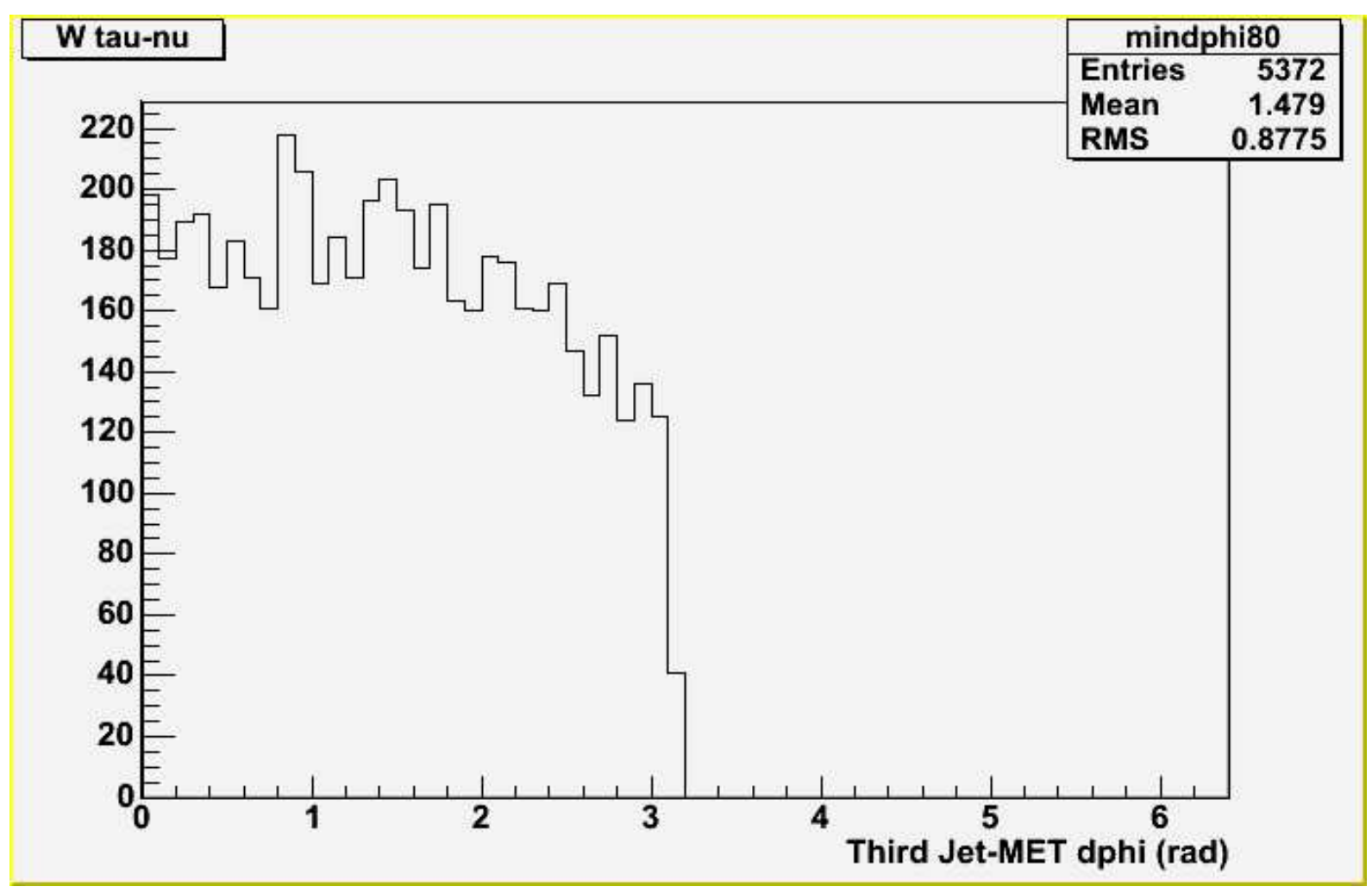

Figure 5.9: $\Delta \phi$ between $\not_{T}$ and the third jet in the event for $W^{-} \rightarrow \tau \bar{\nu}_{\tau}$ events.

Carlo events which pass the low kinematic region cuts correspond to $43 \pm 43$ events when scaled up to the luminosity of the signal sample. This is consistent with the expected $49 \pm 30$ events estimated through data. No events are found to pass the high kinematic cuts in the QCD Monte Carlo sample, which is consistent with the $9 \pm 9$ events estimated from the data.

Finally, the internal consistency of the method was checked by applying the same method used for the data to the PYTHIA QCD Monte Carlo sample, and comparing the predicted number of events in the QCD Monte Carlo which would pass the dijet selection cuts for the low kinematic region. In order to improve statistics, two further tests were carried out with the $E_{T}$ cut lowered to $60 \mathrm{GeV}$ and to $50 \mathrm{GeV}$, but with all the other low kinematic region cuts kept the same. The results for all three $\not_{T}$ cuts are in Table 5.8. For all three tests, the number of QCD dijet events predicted to pass the cuts agrees with the number observed to pass the cuts. 


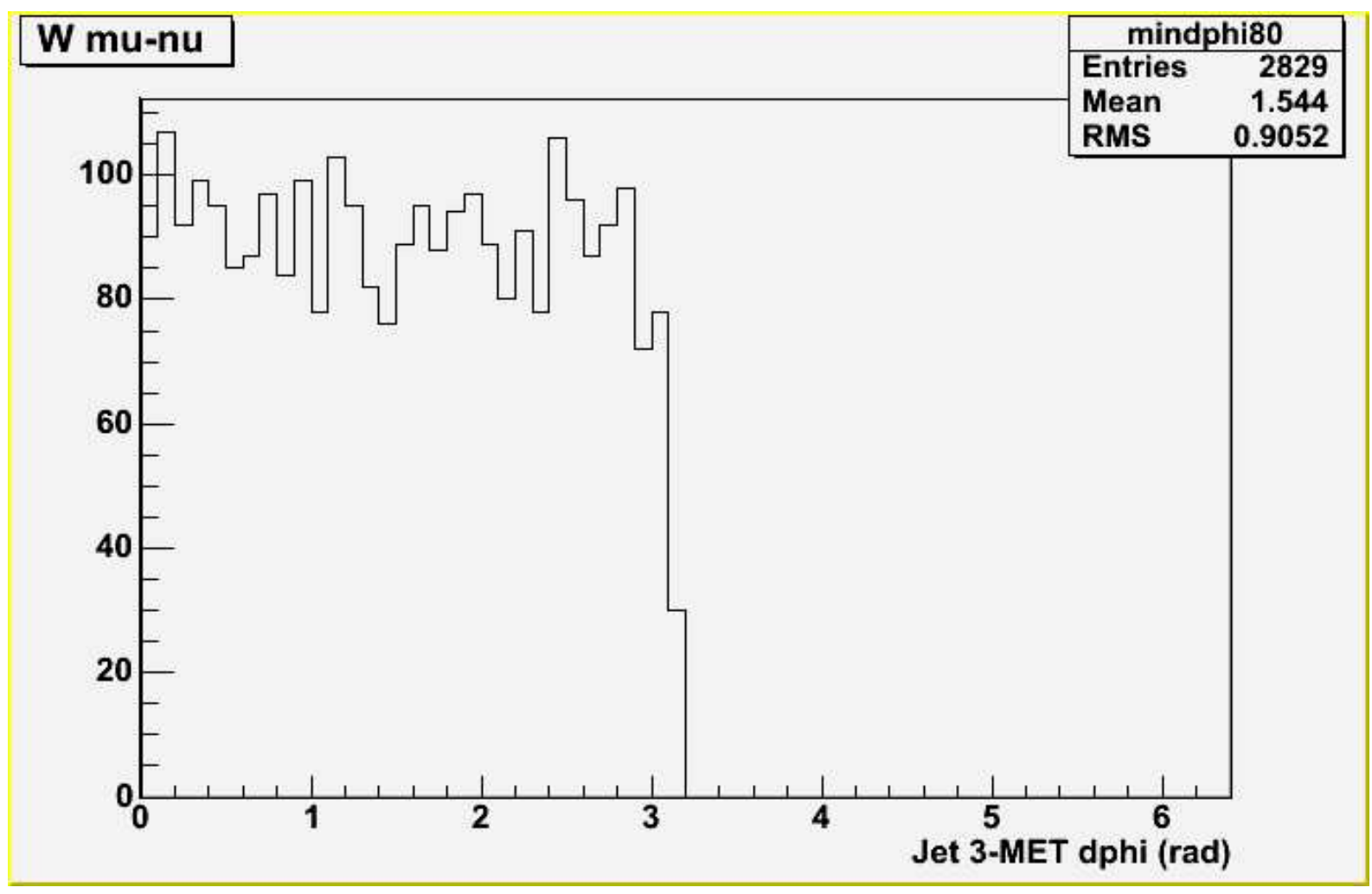

Figure 5.10: $\Delta \phi$ between $\mathbb{E}_{T}$ and the third jet in the event for $W^{-} \rightarrow \mu \bar{\nu}_{\mu}$ events.

\subsection{Other Backgrounds}

There are additional contributions to the background count in the two signal samples. SM processes producing top quarks (singly or in $t \bar{t}$ pairs) or photons associated with jets contribute to the background at levels comparable to the QCD background described in section 5.3. There is also a small contribution from non-collision events.

\subsubsection{Top Backgrounds}

Top quarks decay primarily to a $W$ and a $b$ quark. As previously discussed, $W$ decays may produce final states such as $e \bar{\nu}_{e}, \mu \bar{\nu}_{\mu}$, or $\tau \bar{\nu}_{\tau}$ (with the $\tau$ decaying hadronically or leptonically.) A pair of decaying top quarks, or a single top quark produced by an electroweak interaction involving a $W t b$ vertex, can thus produce a dijet plus $\mathbb{E}_{T}$ signature if any muons or electrons produced are lost or identified as jets, and only two 


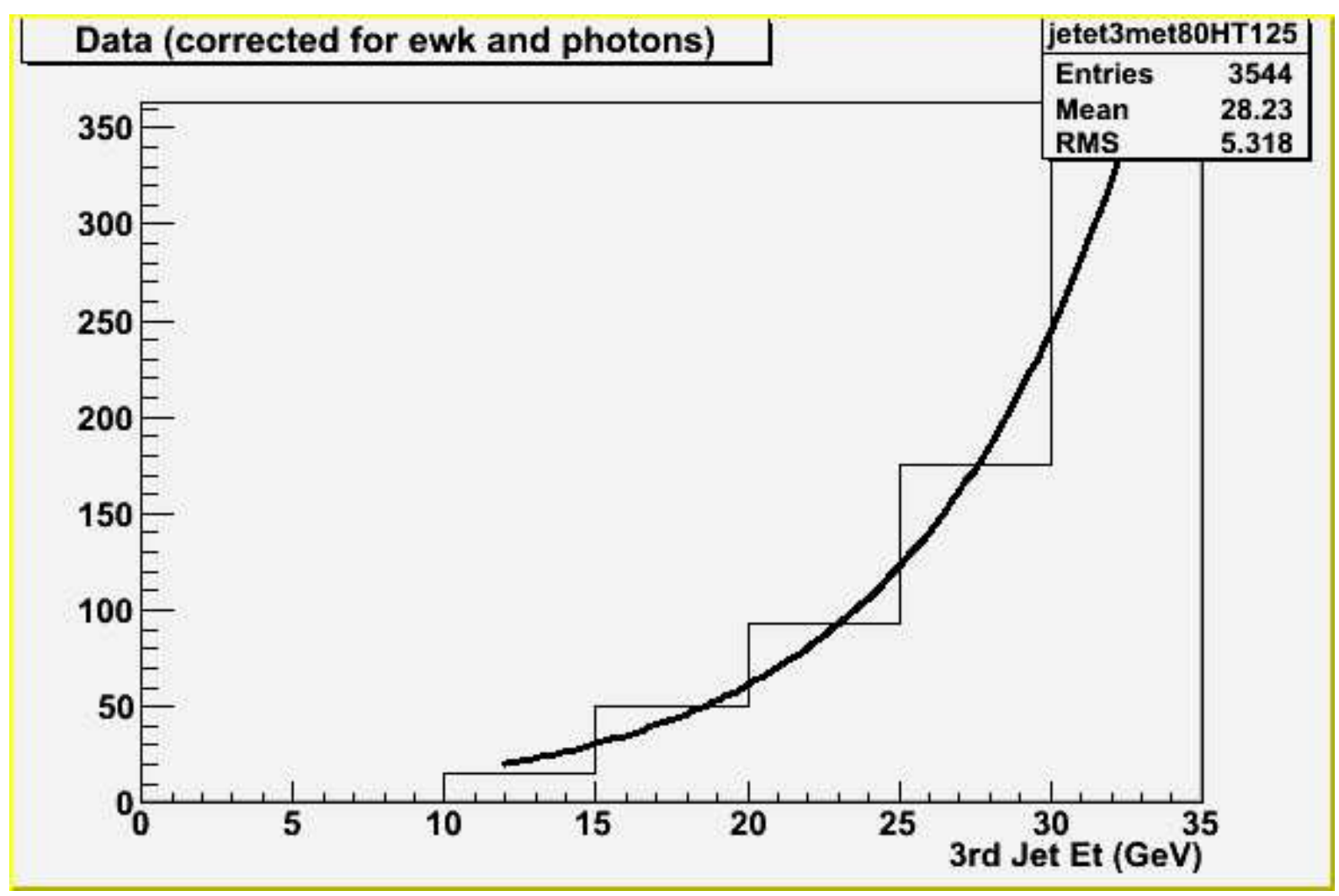

Figure 5.11: Fit to the 3rd jet $E_{T}$ distribution in the data.

jets are counted.

The contributions of both $t \bar{t}$ and single-top production are calculated using PYTHIA Monte Carlo samples. For $t \bar{t}$ production, the results are normalized to a CDF Run II $t \bar{t}$ production cross-section measurement of $7.3 \pm 0.5_{\text {stat }} \pm 0.6_{\text {syst }} \mathrm{pb}$ from $760 \mathrm{pb}^{-1}$ of data [42]. In single top production, a single top quark can be produced through two different channels: $t$-channel processes such as $u+b \rightarrow t+d$ where a virtual space-like $W$ is exchanged, and $s$-channel processes such as $u+\bar{d} \rightarrow t+\bar{b}$ where a virtual time-like $W$ is exchanged ${ }^{3}$. Both $s$ - and $t$-channel components are simulated in PYTHIA. The crosssections used $(0.88 \pm 0.11 \mathrm{pb}$ for the $s$-channel and $1.98 \pm 0.25 \mathrm{pb}$ for the $t$-channel $)$ to normalize the yields come from next-to-leading order (NLO) theoretical calculations [43]. Uncertainties on the cross-sections for both processes, on the jet energy scale, on the top

\footnotetext{
${ }^{3}$ In the $t$-channel case, the $(u, d)$ may be replaced by $(\bar{d}, \bar{u}),(c, s)$, or $(\bar{s}, \bar{c})$. In the $s$-channel case, the $(u, \bar{d})$ pair may be replaced with $(c, \bar{s})$.
} 


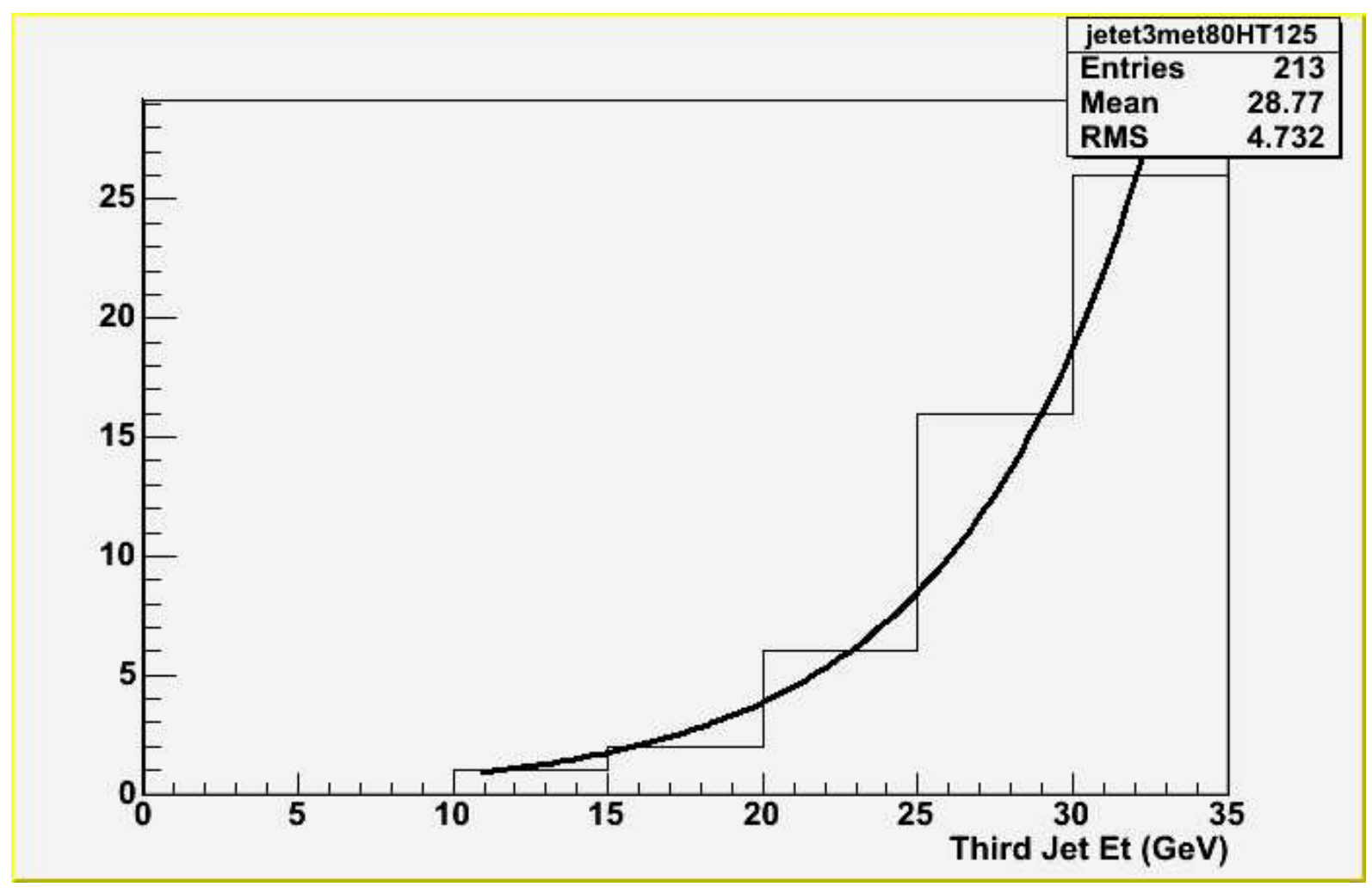

Figure 5.12: Fit to the 3 rd jet $E_{T}$ distribution in the simulation.

quark mass, and on the luminosity are included in the top background estimate.

Table 5.9 presents the predicted background contribution from the three types of top events. The total background contribution from top quark processes is $74 \pm 9$ events in the low kinematic region, and $11 \pm 2$ events in the high kinematic region.

\subsubsection{Photon + Jets Backgrounds}

Another process which can lead to a dijet $+\not_{T}$ signature is the production of two high$E_{T}$ jets with a recoiling photon, where the photon goes undetected. In Run II of DØ the cross-section for the production of a photon with $E_{T}$ between 60 and $300 \mathrm{GeV}$ and $|\eta|<0.9$ was measured to be $136 \pm 17 \mathrm{pb}[44]$. This measurement was scaled up to cover all photons with $E_{T}>60 \mathrm{GeV}$ through a leading order PYTHIA calculation, giving $269 \pm 35 \mathrm{pb}$. 


\begin{tabular}{|c|c|c|}
\hline$E_{T}$ & predicted & observed \\
\hline 50 & 41 & 44 \\
\hline 60 & 12 & 13 \\
\hline 80 & 2 & 2 \\
\hline
\end{tabular}

Table 5.8: Internal consistency check of the QCD background calculation.

\begin{tabular}{|c|c|c|}
\hline Channel & Events (low kinematic region) & Events (high kinematic region) \\
\hline$t \bar{t}$ & $30 \pm 6$ & $6.8 \pm 1.6$ \\
\hline Single top, $s$-channel & $19 \pm 3$ & $2.5 \pm 0.5$ \\
\hline Single top, $t$-channel & $25 \pm 5$ & $1.5 \pm 0.3$ \\
\hline \hline Total & $74 \pm 9$ & $11 \pm 2$ \\
\hline
\end{tabular}

Table 5.9: Single-top and $t \bar{t}$ background contribution.

The acceptance for $\gamma+$ jets events is calculated using a PYTHIA sample generated with a minimum photon $E_{T}$ of $45 \mathrm{GeV}$. All of the regular selection cuts for the signal region, plus a requirement that the photon's $E_{T}$ at the generator level is above $60 \mathrm{GeV}$, are applied. The resulting acceptance, multiplied by the luminosity of the MET45 data sample and the rescaled cross-section, gives the contribution of $\gamma+$ jets events to the total background.

The largest uncertainty in the $\gamma+$ jets contribution comes from the $13.0 \%$ uncertainty in the scaled cross-section. Uncertainties from the acceptance measurement are also included. In the low kinematic region, these are 1.4\% from Monte Carlo statistics, $4.3 \%$ from the jet energy scale uncertainty, and the $6.0 \%$ uncertainty on the Run II luminosity. The final background contribution and uncertainty is $75.0 \pm 11.4$ events in the low kinematic region, and $4.8 \pm 1.1$ events in the high kinematic region.

With all of the background contributions from events produced in $p \bar{p}$ collisions by SM processes predicted, both the low and high kinematic regions in the MET45 data 
sample can be examined. There are 2506 events in the signal candidate sample for the low kinematic region, and 186 in the high kinematic region.

\subsubsection{Non-Collision Backgrounds}

One source of background events remains which has not been estimated through examination of non-signal data or Monte Carlo, however: events from such non-collision sources as cosmic rays and beam-related backgrounds. Though the clean-up cuts described in Section 4.3 get rid of most of these events, some of these events may still survive, particularly those which occur close in time to real collisions in the detector. Three types of non-collision background events are considered: events with out-of-time deposits, events which are flagged as cosmic ray candidates using a track fitting algorithm [36], and events evaluated with the wrong reconstructed vertex.

Timing information from the hadronic calorimeter in the signal candidate events is examined to identify candidate non-collision events. Events with single towers in the hadronic calorimeter which are between 40 ns and 80 ns out-of-time with the nearest collision are searched for. The upper limit in this case avoids tagging events with out-of-time deposits which originate from a previous collision. In the signal candidate sample, seven events are found with out-of-time deposits out of the 2506 events in the low kinematic region. One event with out-of-time deposits out of 186 passes the high kinematic cuts.

To estimate how many of these events are truly non-collision background events, the $W^{-} \rightarrow \mu \bar{\nu}_{\mu}+$ dijet sample is examined. The $W^{-} \rightarrow \mu \bar{\nu}_{\mu}+\operatorname{dijet}$ sample, due to the stringent requirements placed in order to identify $W$ candidates, is expected to have negligible contributions from non-collision background events. This sample is used instead of the $W^{-} \rightarrow e \bar{\nu}_{e}+$ dijet sample, because muons do not appear in the calorimeter. Since electrons appear as jets, they may leave out-of-time deposits in the calorimeter. Since the intention is to compare the number of events with out-of-time deposits from hadronic jets, the $W^{-} \rightarrow \mu \bar{\nu}_{\mu}+$ jets sample is a fairer comparison to the signal candidate sample. 
One event out of 363 in the low kinematic region from the $W^{-} \rightarrow \mu \bar{\nu}_{\mu}+$ dijet sample is identified as having out-of-time deposits. Scaling this up to the 2506 events found in the low kinematic region in the signal sample results in 6.9 expected events with out-of-time deposits which would be expected to come from $p \bar{p}$ events. In the high kinematic region, no events with out-of-time deposits are found in the $W^{-} \rightarrow \mu \bar{\nu}_{\mu}+$ dijet sample.

A cosmic track fitting algorithm [36] uses COT timing information for all COT tracks with $p_{T}>10 \mathrm{GeV} / c$ to check for cosmic ray candidates which pass through both hemispheres of the detector. If, for any of the tracks, the algorithm finds a good fit for either an outgoing or incoming cosmic ray with $\chi^{2}<1000$ for the fit, the event is tagged as a cosmic ray candidate. This happens for three events passing the low kinematic region cuts in the signal candidate sample (a fraction of $0.120 \pm 0.007 \%$ ), and for no events in the high kinematic region. By comparison, in the $W^{-} \rightarrow e \bar{\nu}_{e}+$ dijet sample, where the $W$ selection criteria should ensure no contamination from cosmic rays, two out of 508 events (or $0.39 \pm 0.27 \%$ ) can be tagged. Since the tagging rate in the signal candidate sample is lower than that in the $W^{-} \rightarrow e \bar{\nu}_{e}+$ dijet sample which is presumed to be cosmic-free, the cosmic ray candidates in the signal candidate sample are presumed to be beamproduced, and not added to the non-collision background estimate for the low kinematic region. For the high kinematic cuts, no events are tagged as cosmic ray candidates in either sample, so there is again nothing added to the non-collision background estimate. The $W^{-} \rightarrow \mu \bar{\nu}_{\mu}+$ dijet sample is not used for this comparison because events identified by the cosmic track fitting algorithm are removed from the high- $p_{T}$ muon sample as part of the $W$ identification process (see Subsection 5.2.1.)

Finally, it is possible that in events with multiple reconstructed vertices, the jet $E_{T} \mathrm{~s}$ are calculated using the wrong vertex. This could lead to events passing the kinematic cuts based on an incorrect evaluation of $\not_{T}$ and $H_{T}$. The five events in the low kinematic region where the vertex used in event selection is not the same as the one used in jet clustering are examined using a CDF event display program. This program graphs the 
$E_{T}$ deposition in each calorimeter tower and the track locations for each event. In all five events, the vertex used for jet clustering is indeed the vertex where the high- $E_{T}$ jets selected actually came from. These events are therefore considered to be good signal candidate events, and are left in the candidate sample. With no contribution from this or from cosmic ray candidates, the non-collision background is estimated entirely from the events with out-of-time deposits.

In the low kinematic region, the single $W^{-} \rightarrow \mu \bar{\nu}_{\mu}$ event with an out-of-time deposit is assumed to be a statistical fluctuation. In other words, the expectation is that $1 \pm 1$ events out of 363 total would have out-of-time deposits despite coming from collisions. If the true value were either one or two events, the expected number of events would scale up to 6.9 or 13.8 events in the signal sample, which would be consistent with the seven events with out-of-time deposits found in the signal sample all being beam-produced. If the true value were zero, the seven events in the signal sample would all be presumed to be non-collision backgrounds. Thus, since the comparison to the $W^{-} \rightarrow \mu \bar{\nu}_{\mu}$ sample is consistent with anywhere from none to all seven of the events with out-of-time deposits in the signal sample being non-collision backgrounds, the non-collision background is taken to be $3.5 \pm 3.5$, or $4 \pm 4$ events. In the high kinematic region, only one event is seen in the signal sample, while none are expected from the $W^{-} \rightarrow l \bar{\nu}_{l}$ comparisons. This is taken to be a non-collision contribution of $1 \pm 1$ events.

\subsection{Summary}

Table 5.10 summarizes the total background contributions from each SM process, together with the non-collision background and the number of events actually observed. The relative contributions to the SM background for the low kinematic region are also shown in Figures 5.13 and 5.14, which show the stacked contributions of each SM process as a function of $\not_{T}$ and $H_{T}$ respectively. 


\begin{tabular}{|l|c|c|}
\hline Background & $H_{T}>125, F_{T}>80$ & $H_{T}>225, F_{T}>100$ \\
\hline \hline$Z \rightarrow \nu \bar{\nu}$ & $888 \pm 54$ & $86 \pm 13$ \\
\hline$W^{-} \rightarrow \tau \bar{\nu}_{\tau}$ & $669 \pm 42$ & $50 \pm 8$ \\
\hline$W^{-} \rightarrow \mu \bar{\nu}_{\mu}$ & $399 \pm 25$ & $33 \pm 5$ \\
\hline$W^{-} \rightarrow e \bar{\nu}_{e}$ & $256 \pm 16$ & $14 \pm 2$ \\
\hline$Z \rightarrow l \bar{l}$ & $29 \pm 4$ & $2 \pm 0$ \\
\hline Top Quark Production & $74 \pm 9$ & $11 \pm 2$ \\
\hline QCD & $49 \pm 30$ & $9 \pm 9$ \\
\hline$\gamma+$ jet & $75 \pm 11$ & $5 \pm 1$ \\
\hline Non-collision & $4 \pm 4$ & $1 \pm 1$ \\
\hline \hline Total predicted & $2443 \pm 145$ & $211 \pm 30$ \\
\hline Data observed & 2506 & 186 \\
\hline
\end{tabular}

Table 5.10: Summary of estimated SM background contributions to the dijet plus $\not_{T}$ candidate samples along with the number of observed events in data. 


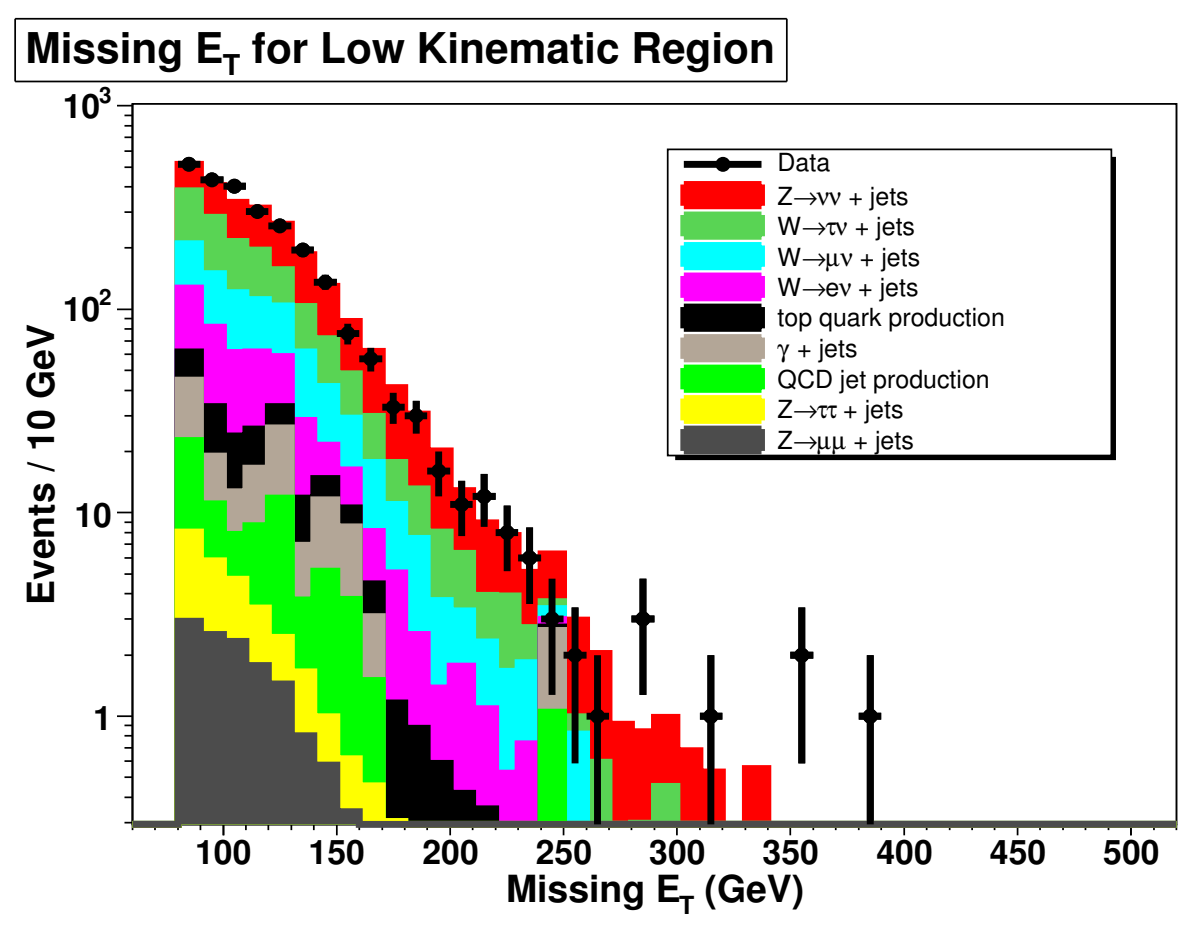

Figure 5.13: Comparison of the event missing $E_{T}$ distribution for the 2506 events in the low kinematic region candidate sample with the predicted SM distribution showing the stacked contributions of each SM background process.

It is important to note that this analysis is sensitive to the number of events in the two signal regions rather than the shape of any kinematic distributions. Comparisons can still be made between the expected background distribution and the results found in the data. Figures 5.15 through 5.21 compare the distributions of $E_{T}, H_{T}$, individual jet $E_{T}$ and $\eta$, and the $\Delta \phi$ between the two jets found in the data with the predicted SM background distribution in the low kinematic region. Figures 5.22 and 5.23 also compare $\not_{T}$ and $H_{T}$ data and $\mathrm{SM}$ predictions for the low kinematic regions, but with the SM prediction normalized to the number of events actually seen in the data rather than the predicted number of events. This allows direct shape comparisons. The agreement between data and SM prediction in the low kinematic region is reasonable in these figures.

For the high kinematic region, Figures 5.24 through 5.30 also compare the distributions of $E_{T}, H_{T}$, individual jet $E_{T}$ and $\eta$, and the $\Delta \phi$ between the two jets found in 


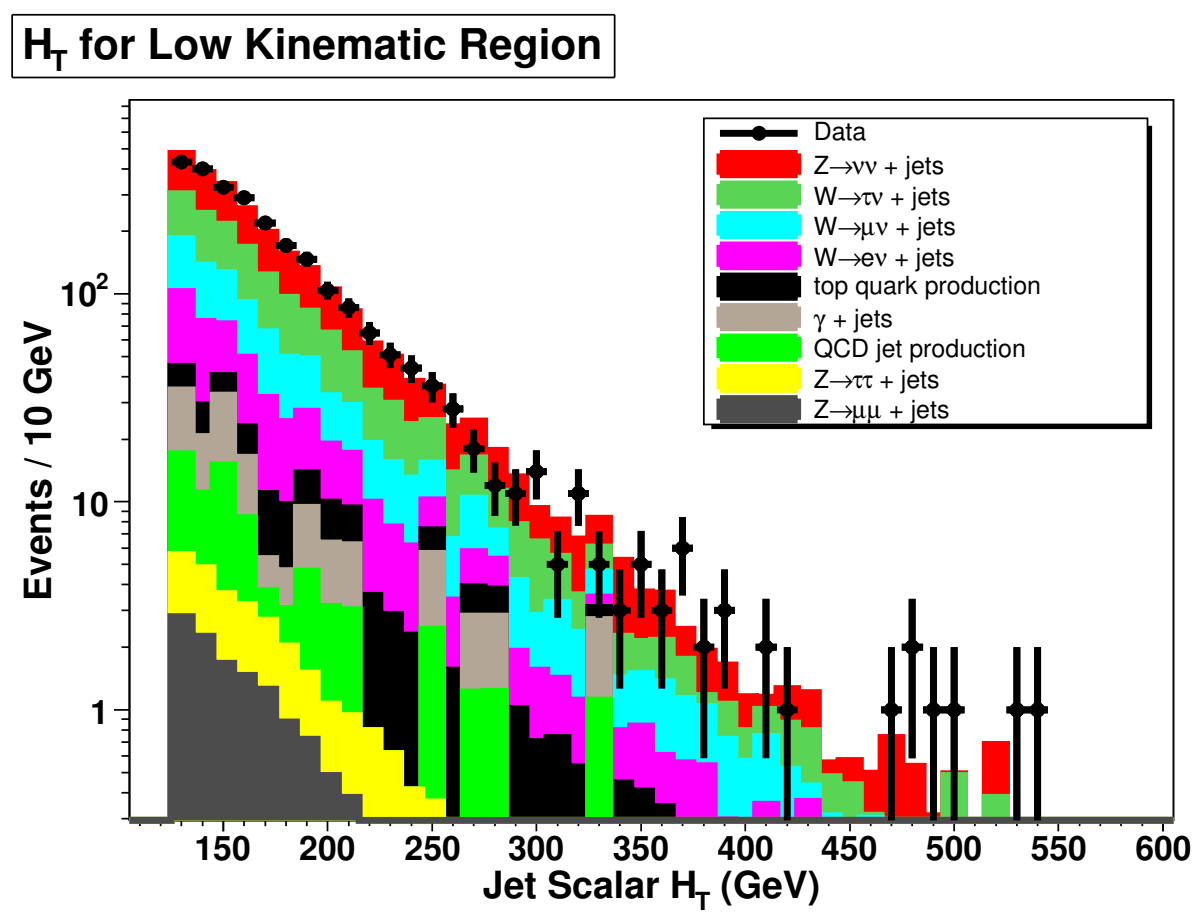

Figure 5.14: Comparison of the combined jet $H_{T}$ distribution for the 2506 events in the low kinematic region candidate sample with the predicted SM distribution showing the stacked contributions of each SM background process.

the data with the predicted SM background distribution. Again, the agreement for these kinematic distributions is reasonable.

Figure 5.31 compares $H_{T}$ distributions for events passing all cuts in the high kinematic region, but with the $H_{T}>225 \mathrm{GeV}$ cut ignored. In this figure, the $H_{T}$ distribution associated with each background is normalized to its estimated contribution within the high kinematic signal region in order to get the total Monte Carlo prediction. 


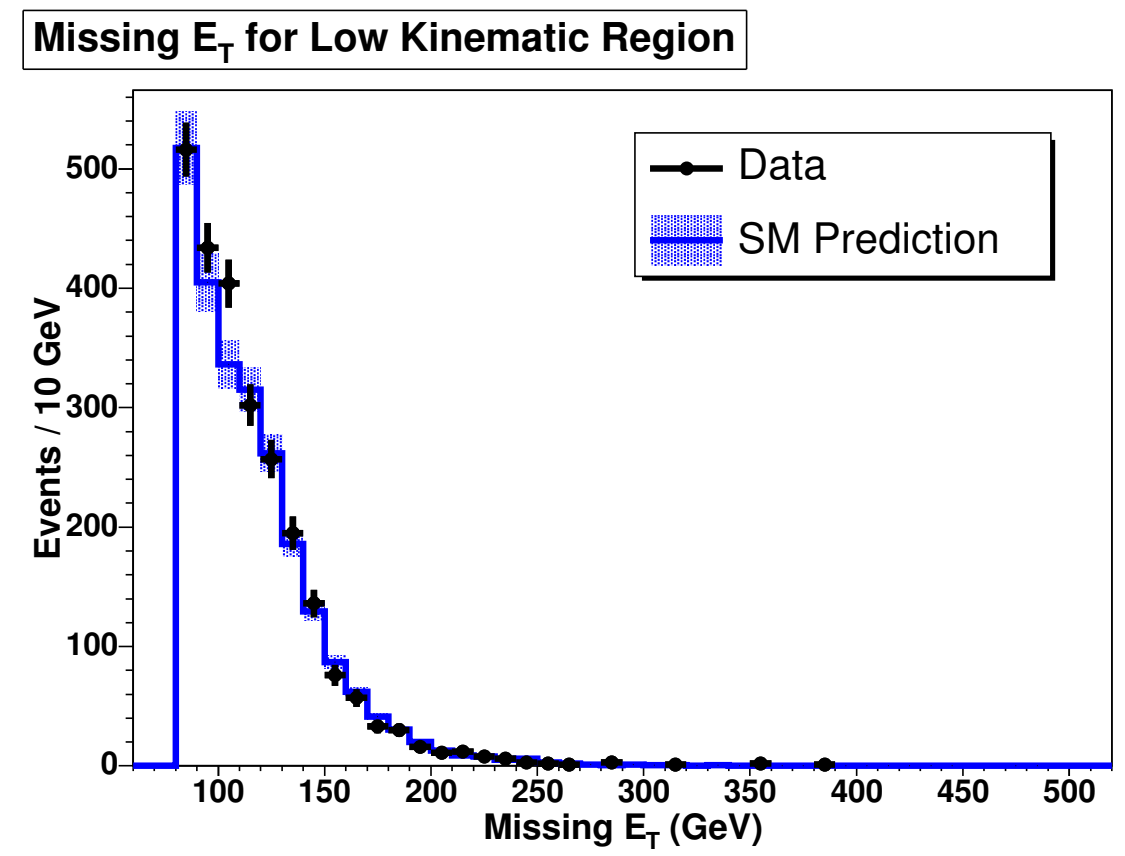

Figure 5.15: Comparison of the $\not_{T}$ distribution for the 2506 events in the low kinematic region candidate sample with the predicted SM distribution.

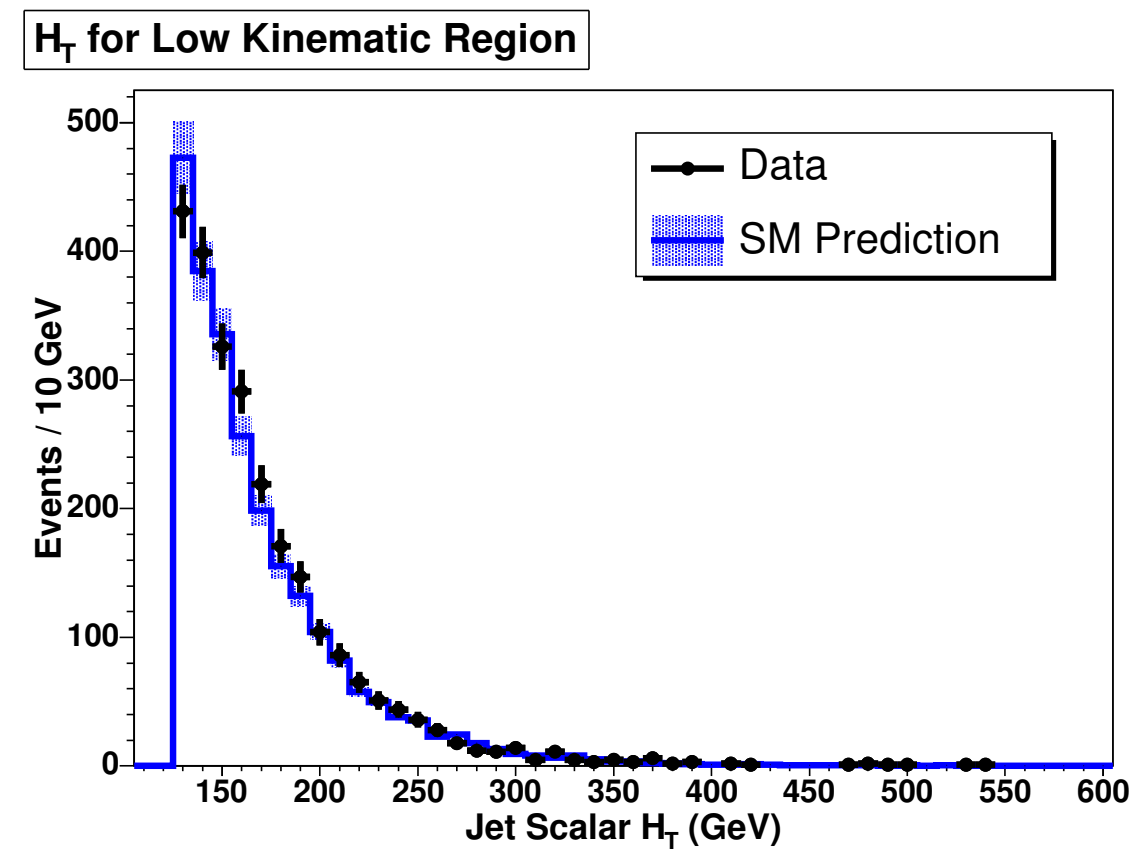

Figure 5.16: Comparison of the $H_{T}$ distribution for the 2506 events in the low kinematic region candidate sample with the predicted SM distribution. 


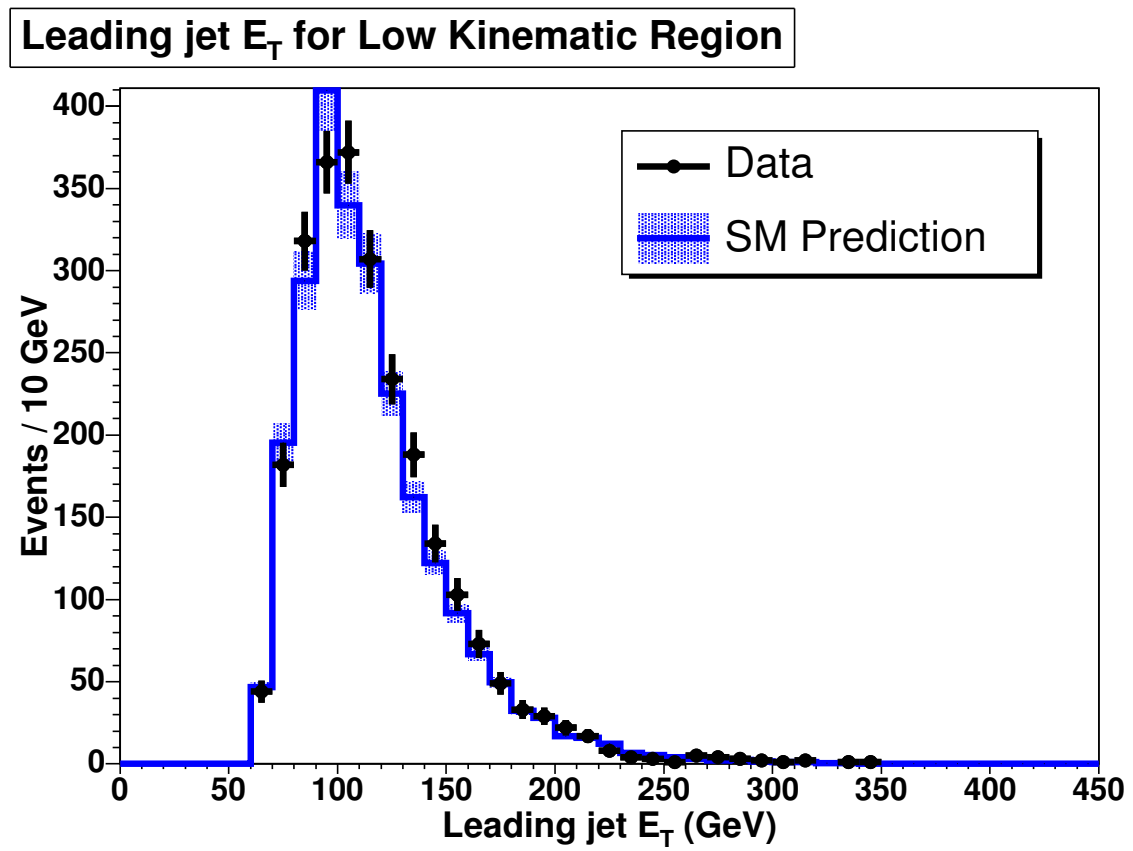

Figure 5.17: Comparison of the leading jet $E_{T}$ distribution for the 2506 events in the low kinematic region candidate sample with the predicted SM distribution.

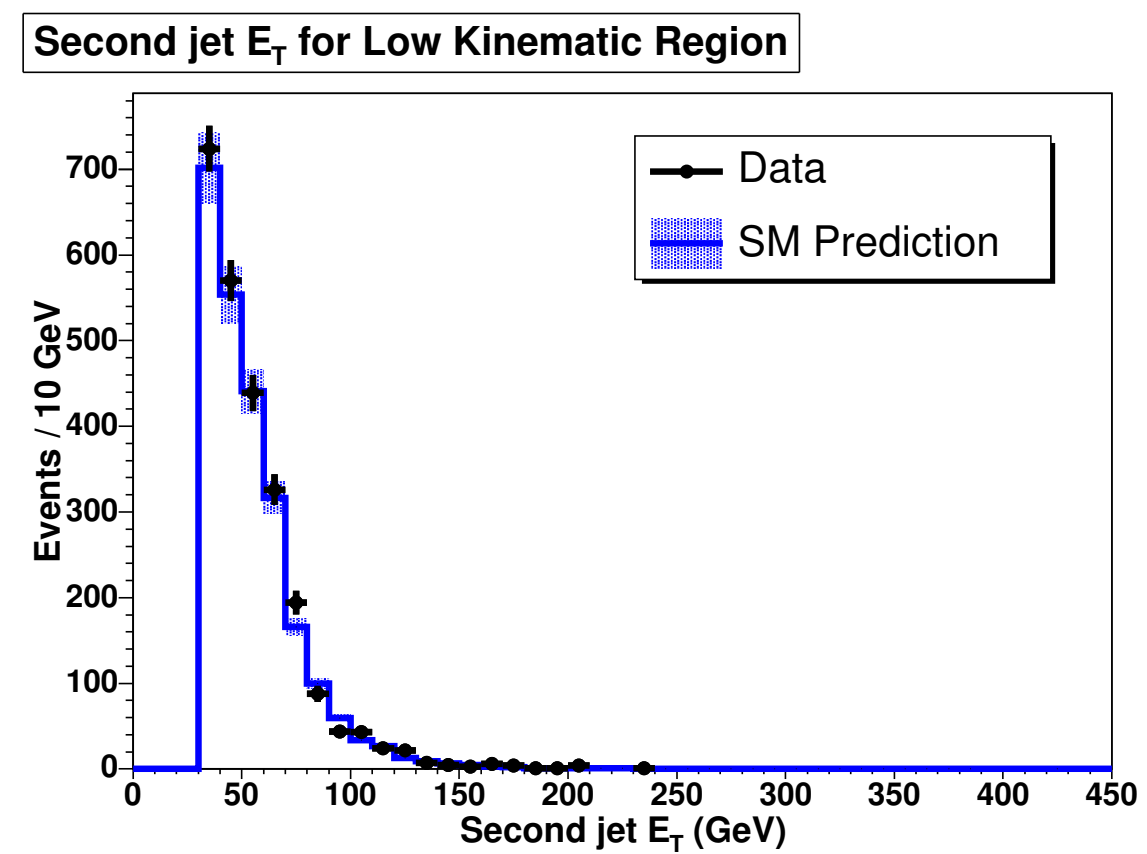

Figure 5.18: Comparison of the second jet $E_{T}$ distribution for the 2506 events in the low kinematic region candidate sample with the predicted SM distribution. 


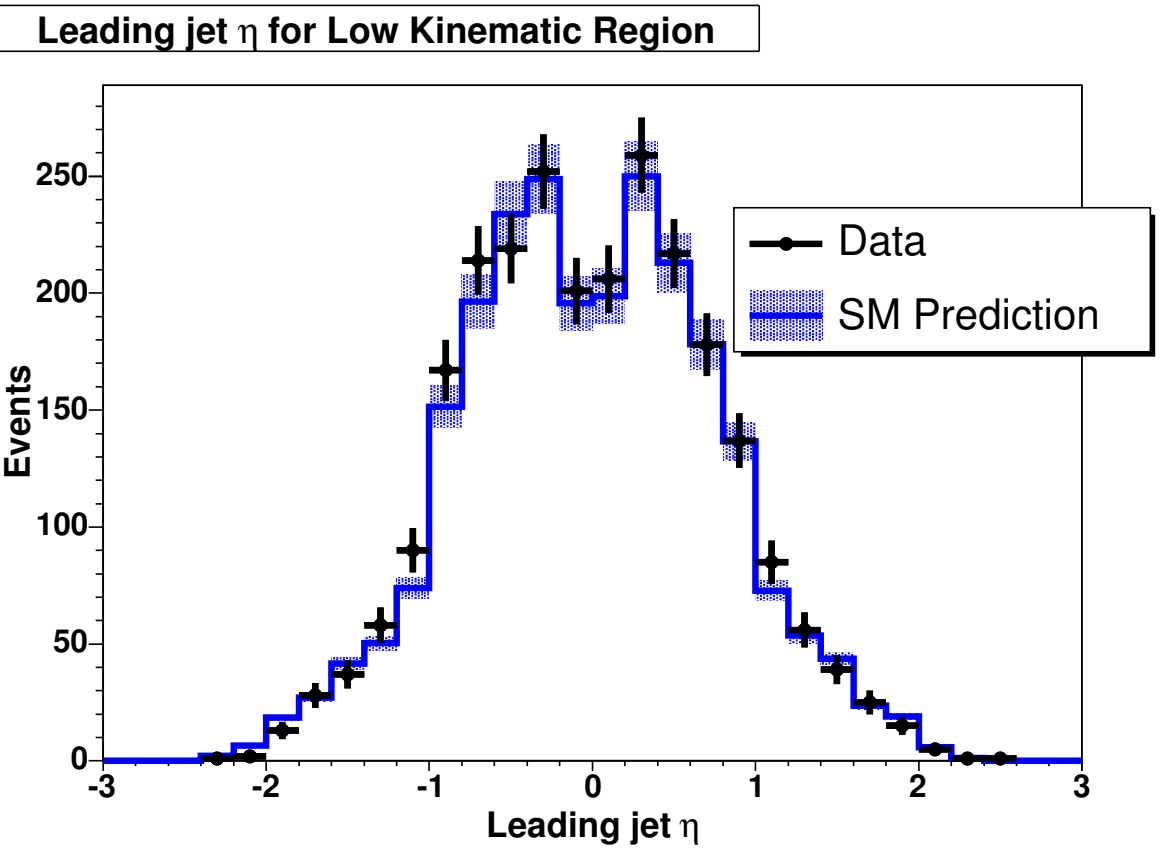

Figure 5.19: Comparison of the leading jet $\eta$ distribution for the 2506 events in the low kinematic region candidate sample with the predicted SM distribution.

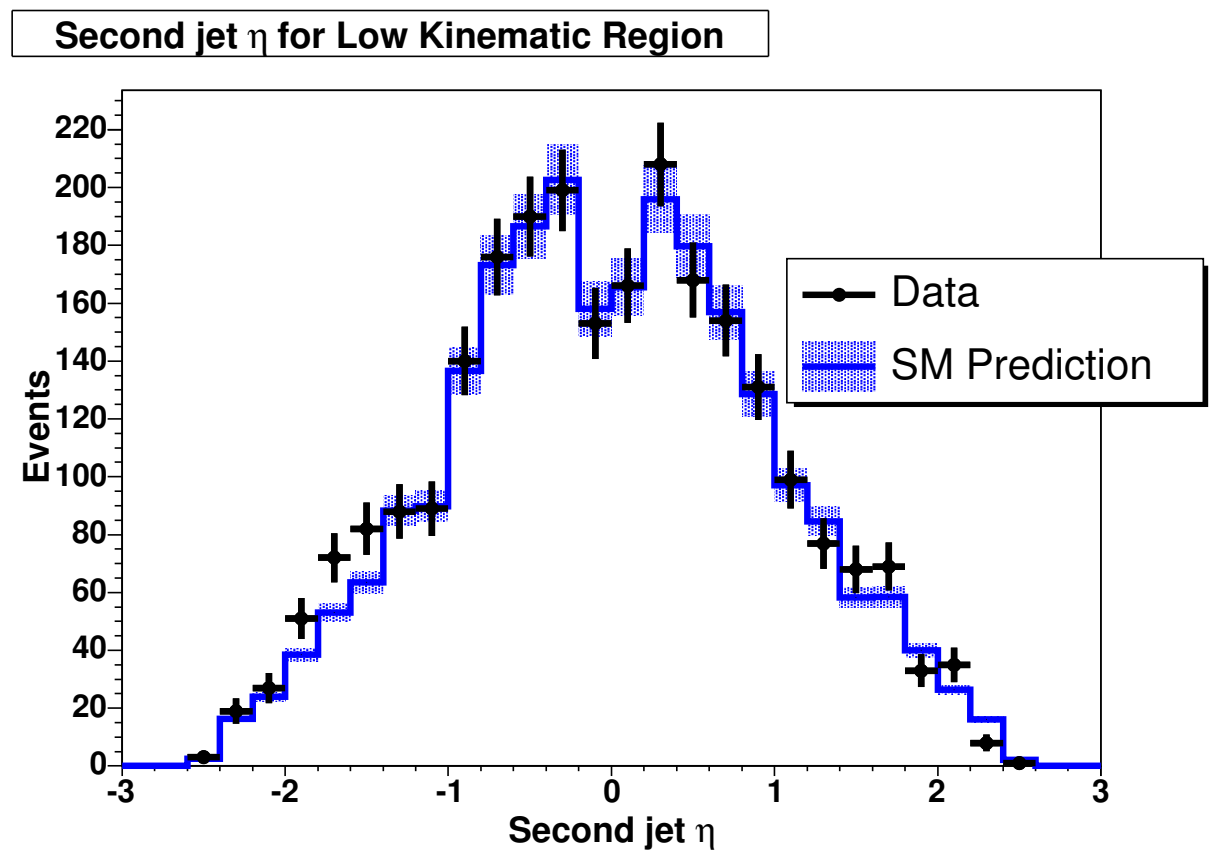

Figure 5.20: Comparison of the second jet $\eta$ distribution for the 2506 events in the low kinematic region candidate sample with the predicted SM distribution. 


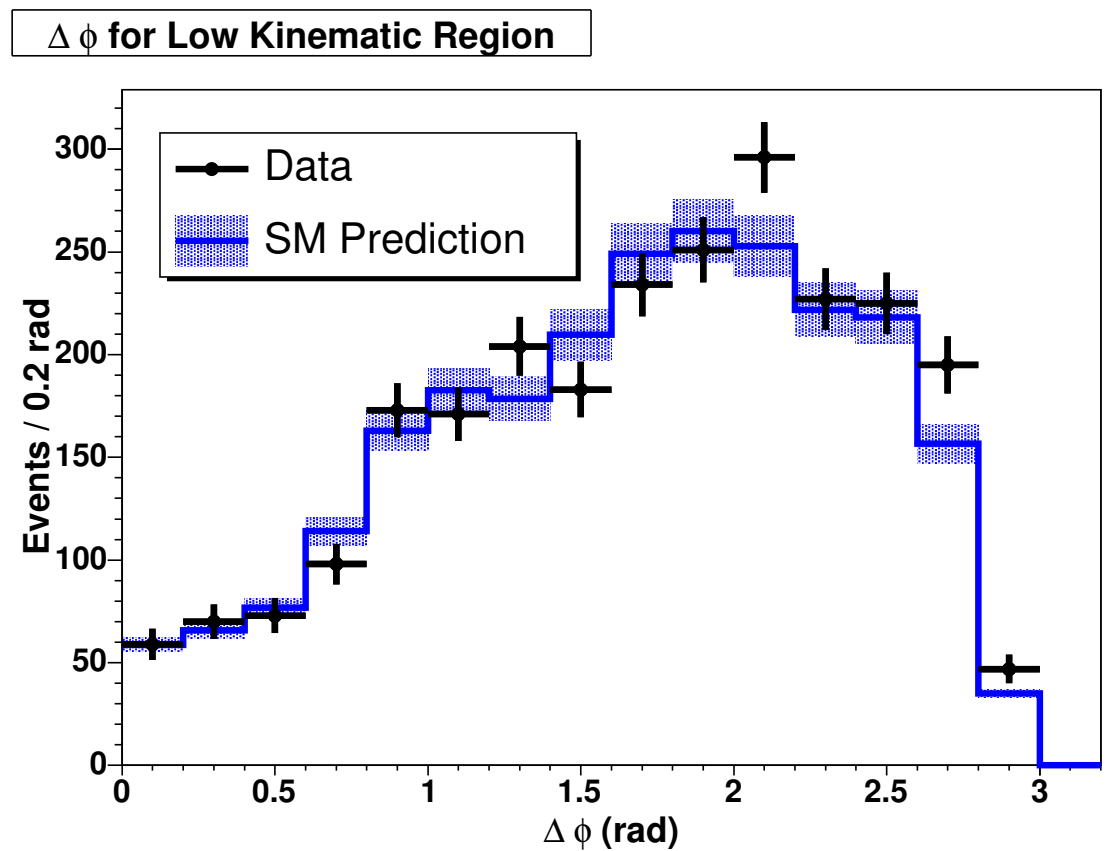

Figure 5.21: Comparison of the distribution for $\Delta \phi$ between the two jets for the 2506 events in the low kinematic region candidate sample with the predicted SM distribution.

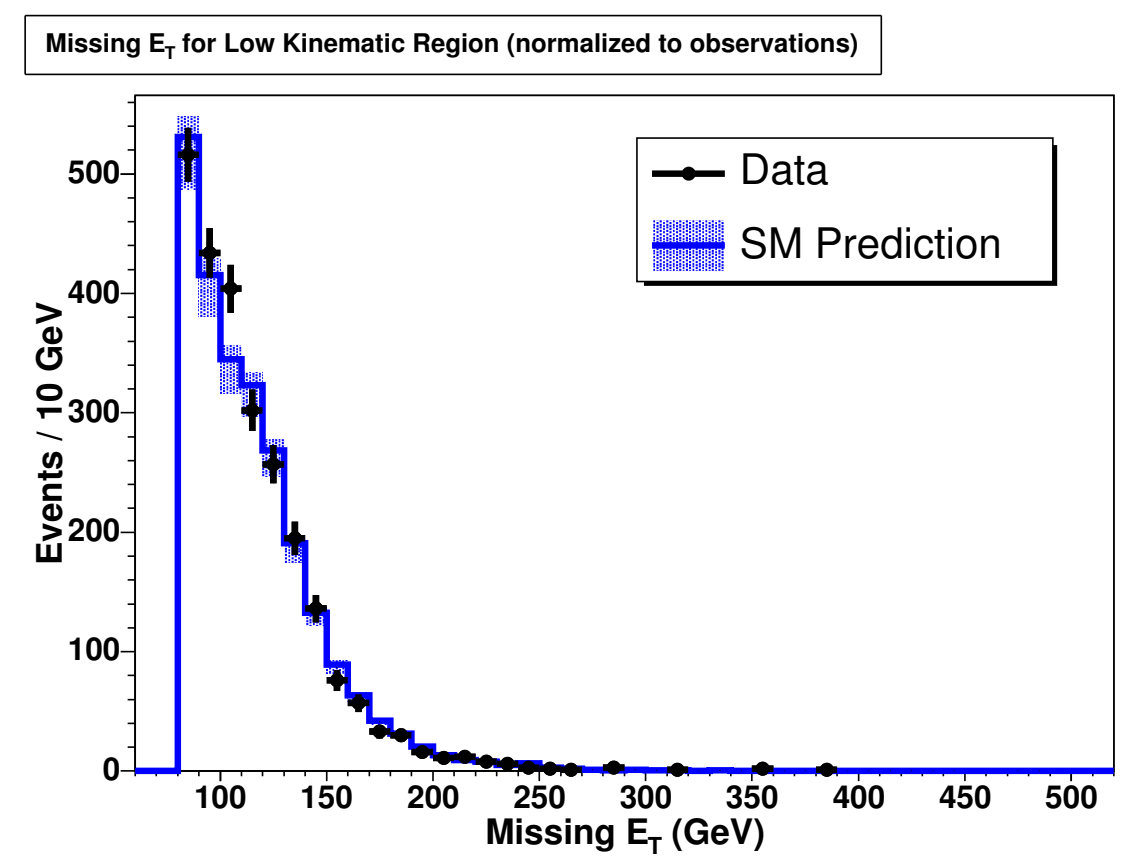

Figure 5.22: Shape comparison of the $E_{T}$ distribution between data and the SM prediction in the low kinematic region. The combined Monte Carlo shape is normalized to match the 2506 observed events in data. 


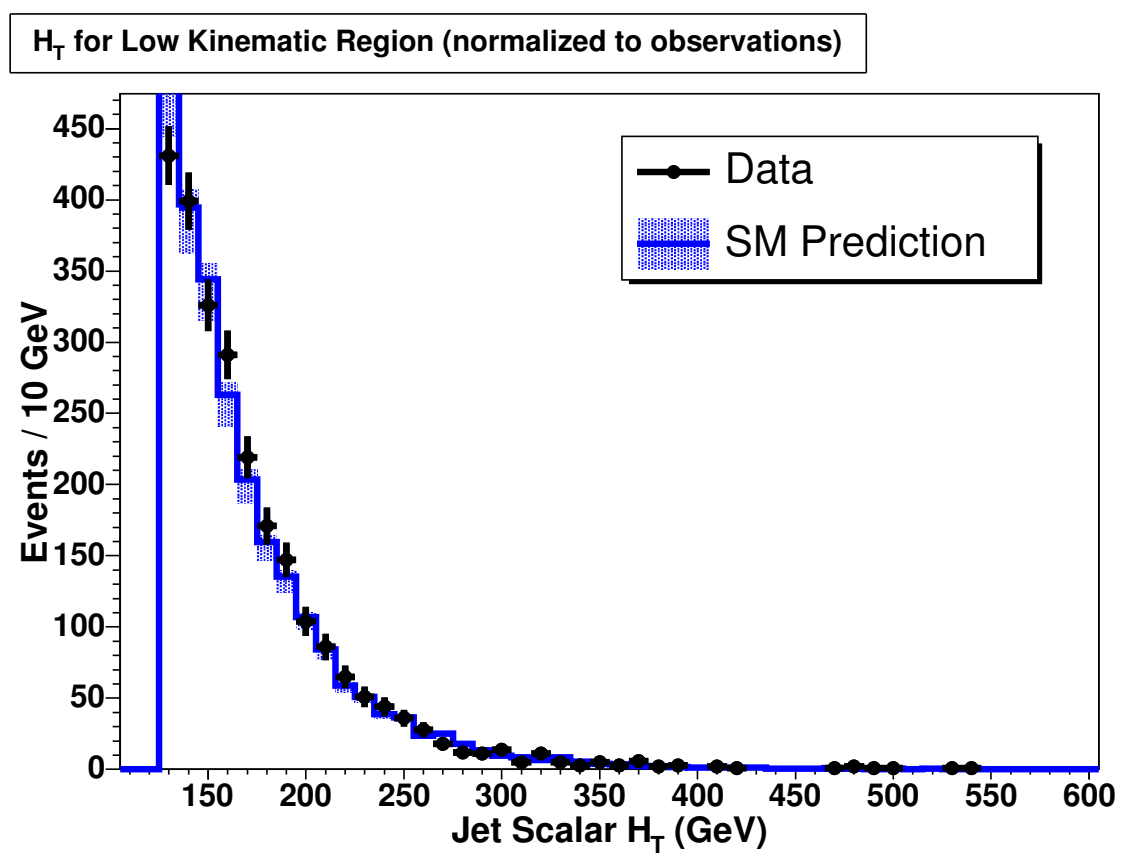

Figure 5.23: Shape comparison of the $H_{T}$ distribution between data and the SM prediction in the low kinematic region. The combined Monte Carlo shape is normalized to match the 2506 observed events in data.

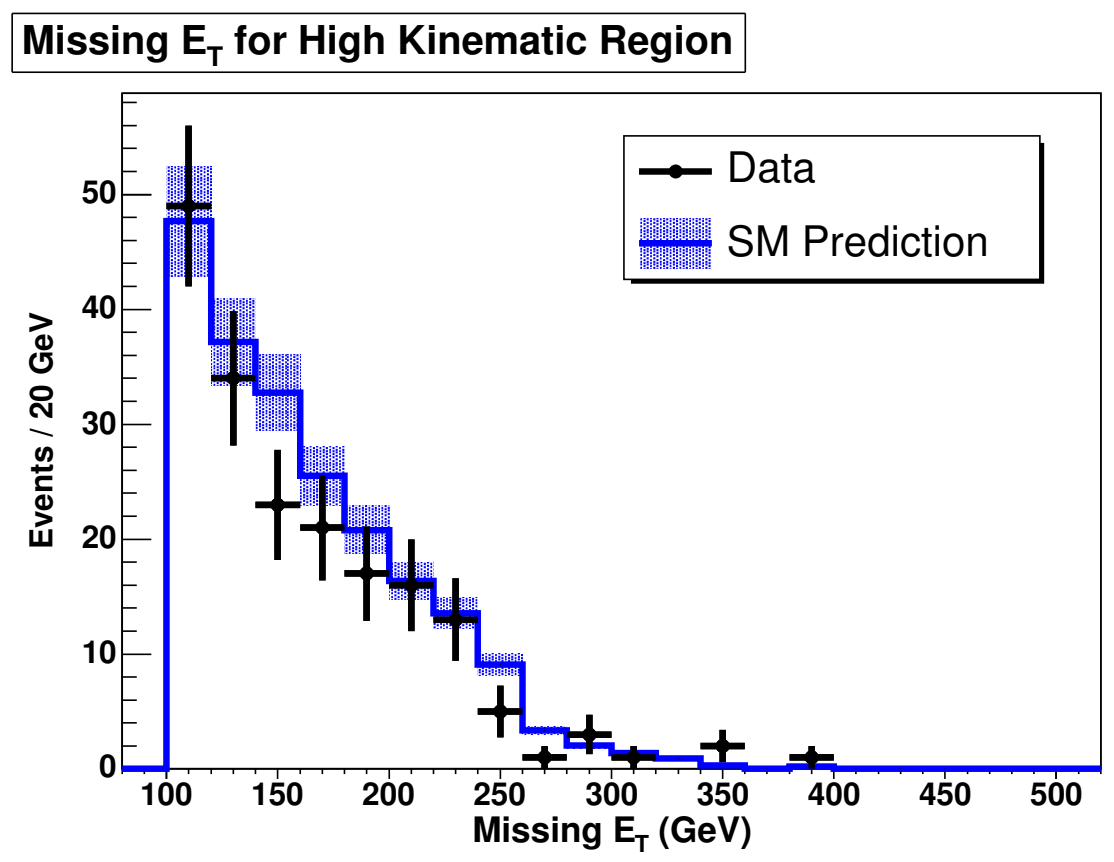

Figure 5.24: Comparison of the $\mathbb{E}_{T}$ distribution for the 186 events in the high kinematic region candidate sample with the predicted SM distribution. 


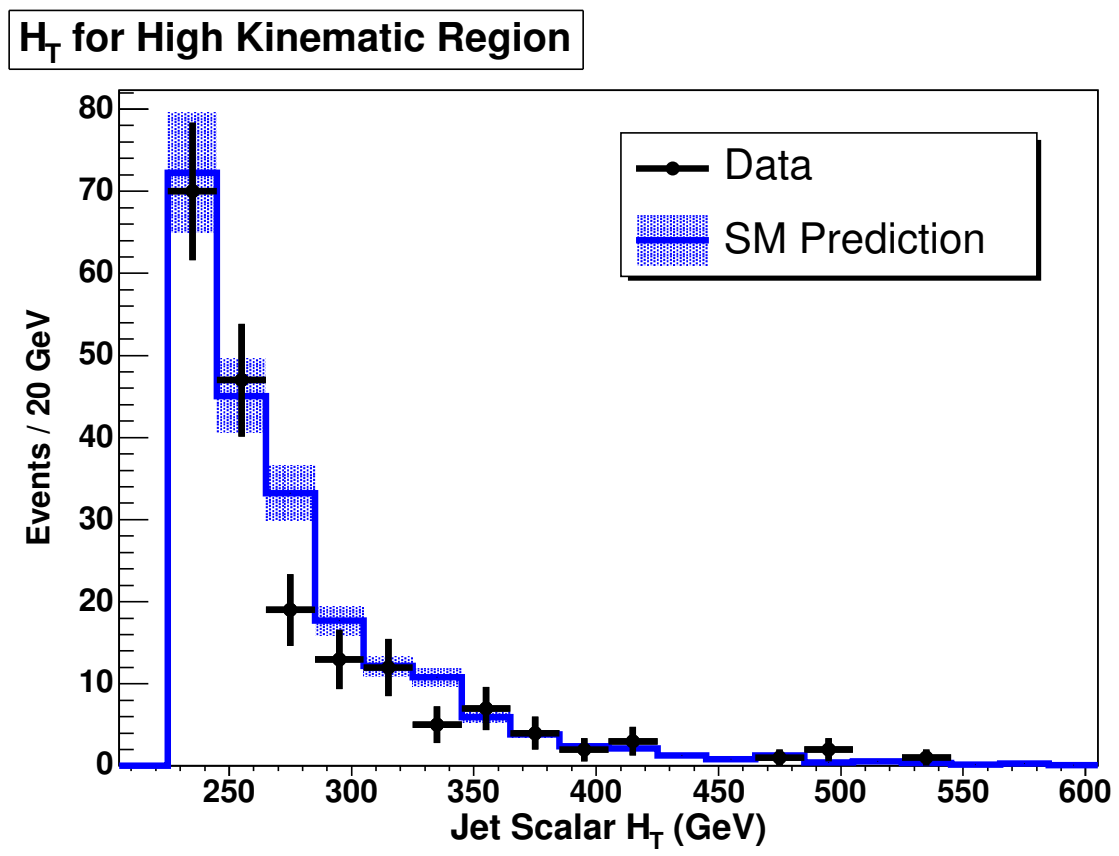

Figure 5.25: Comparison of the $H_{T}$ distribution for the 186 events in the high kinematic region candidate sample with the predicted SM distribution.

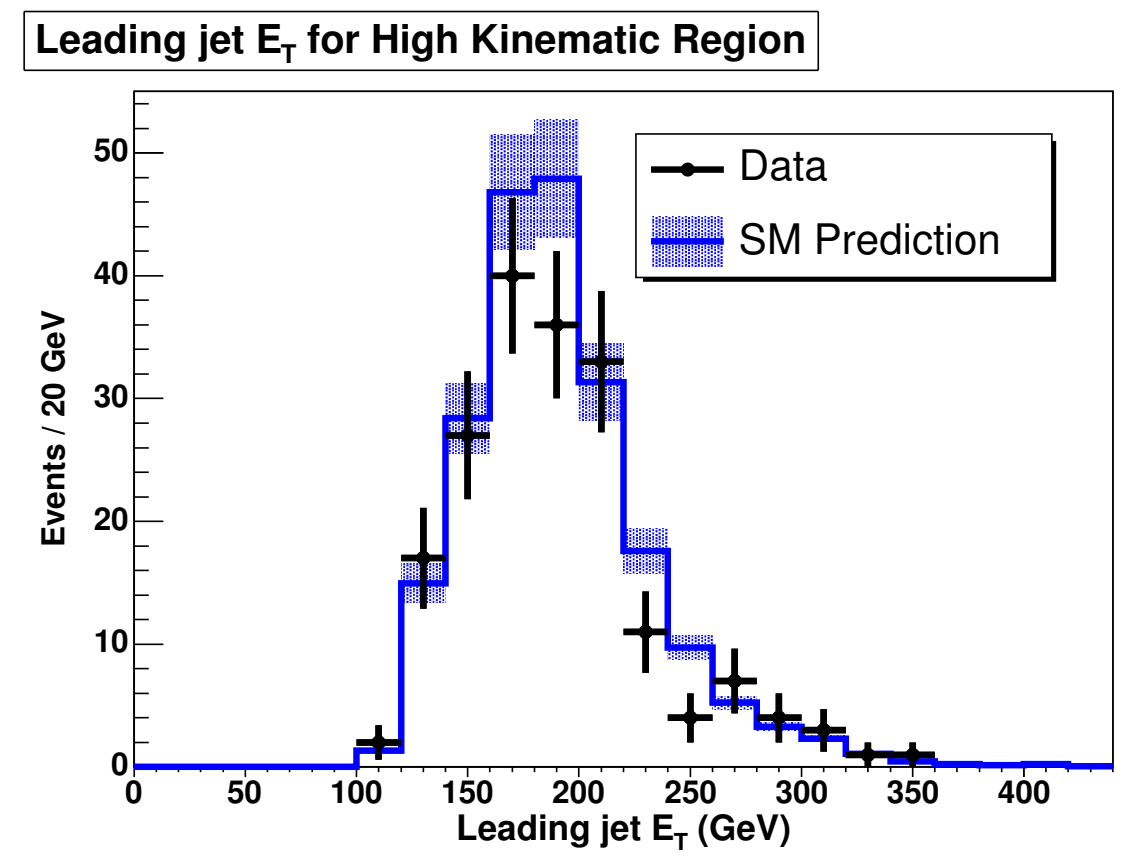

Figure 5.26: Comparison of the leading jet $E_{T}$ distribution for the 186 events in the high kinematic region candidate sample with the predicted SM distribution. 


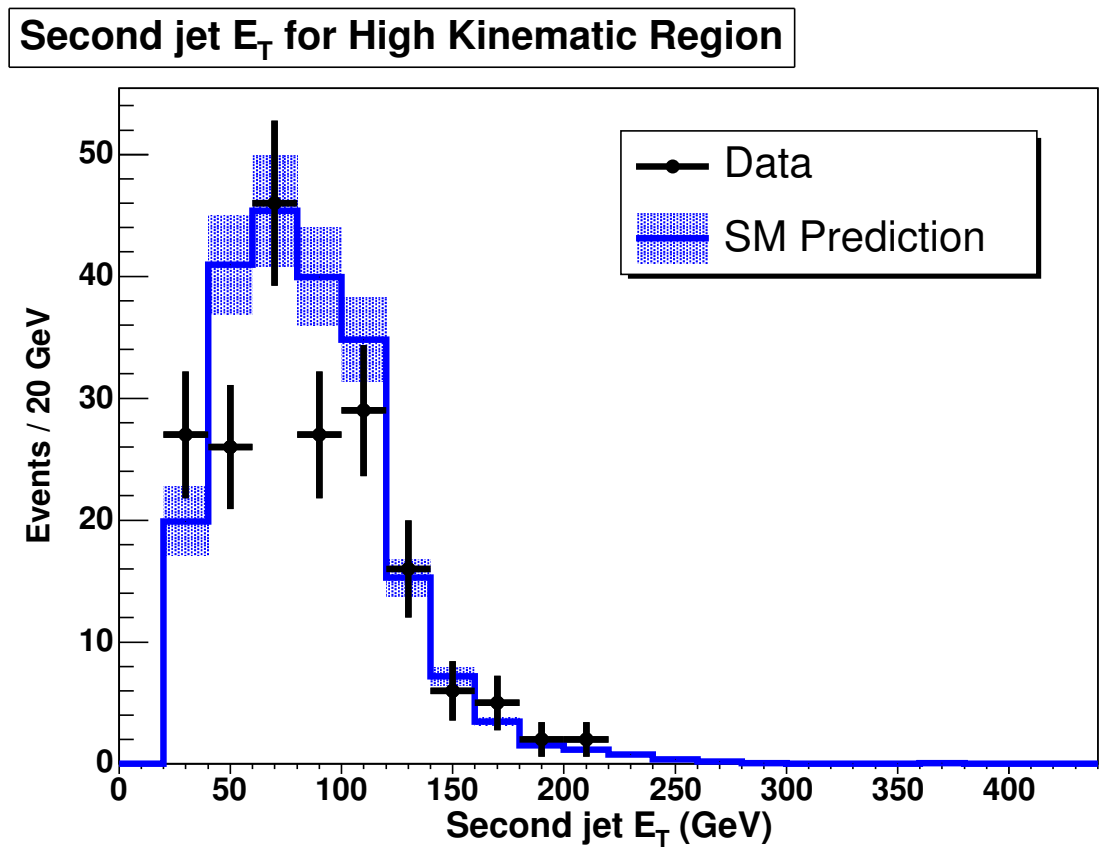

Figure 5.27: Comparison of the second jet $E_{T}$ distribution for the 186 events in the high kinematic region candidate sample with the predicted SM distribution.

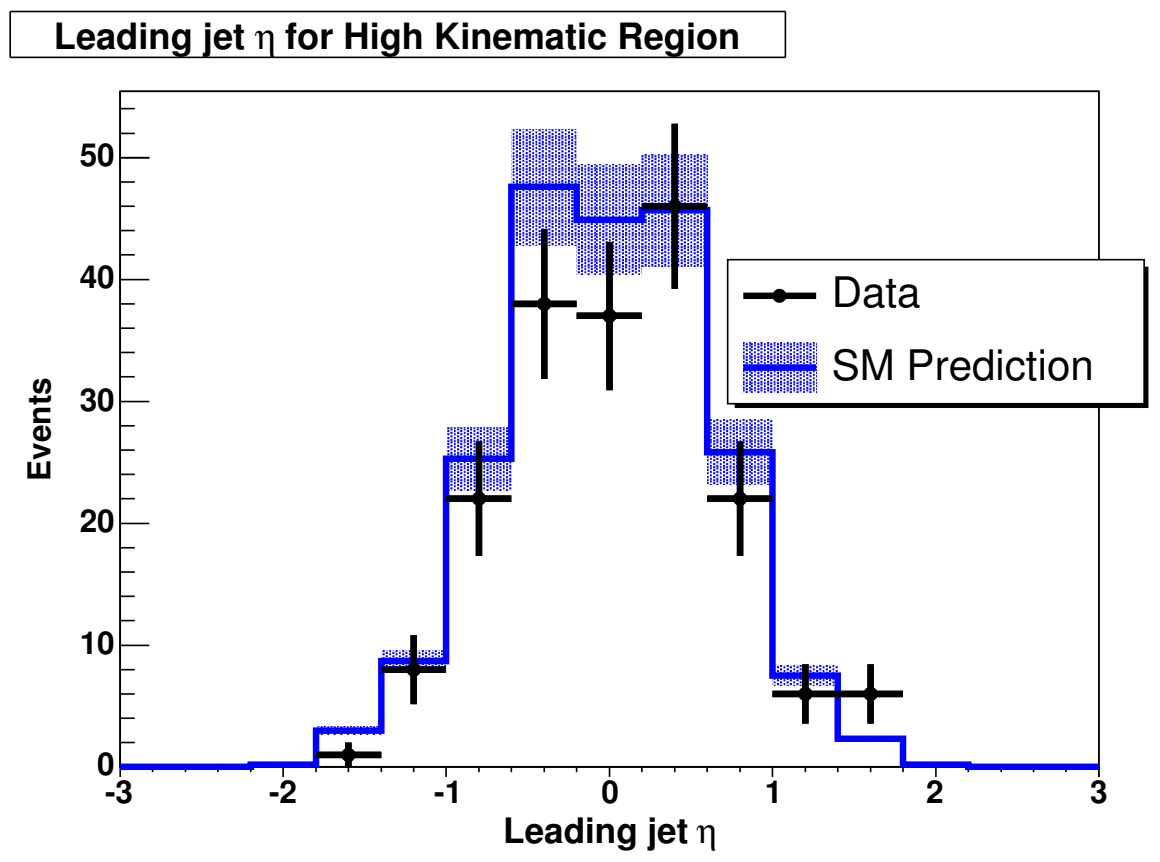

Figure 5.28: Comparison of the leading jet $\eta$ distribution for the 186 events in the high kinematic region candidate sample with the predicted SM distribution. 


\section{Second jet $\eta$ for High Kinematic Region}

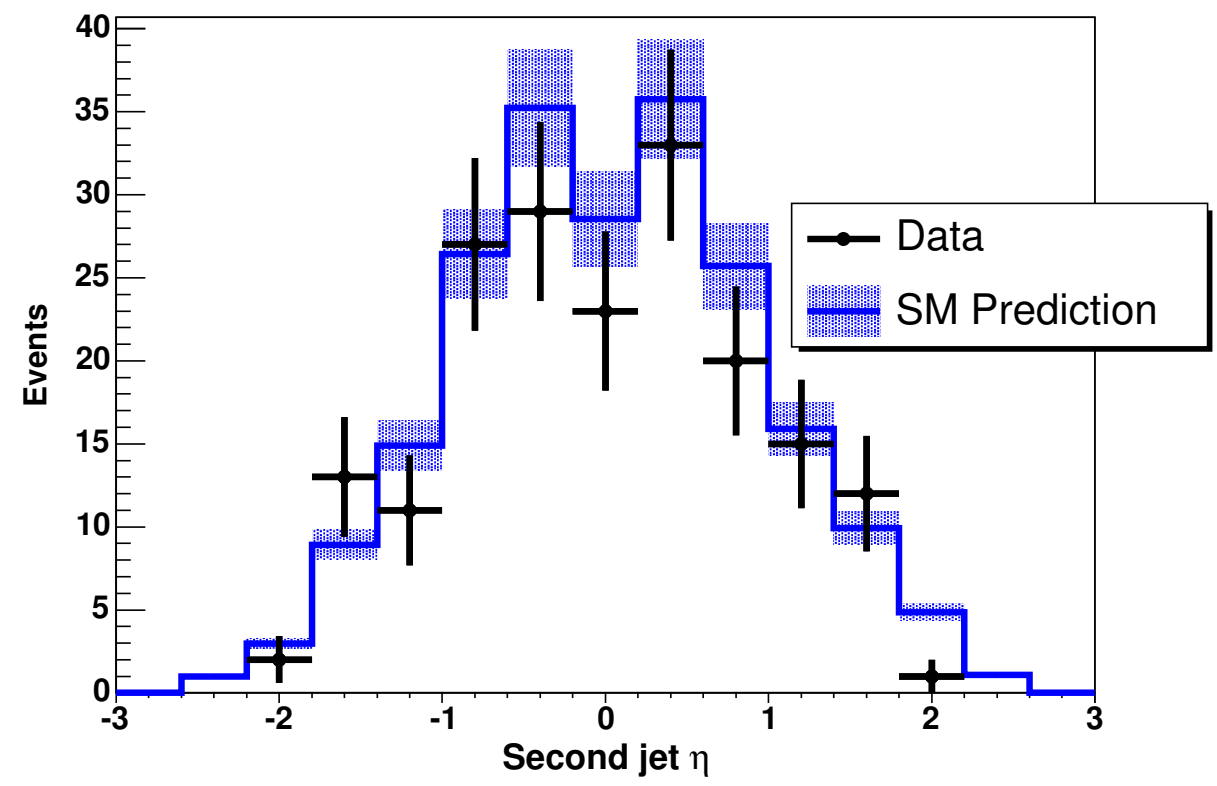

Figure 5.29: Comparison of the trailing jet $\eta$ distribution for the 186 events in the high kinematic region candidate sample with the predicted SM distribution.

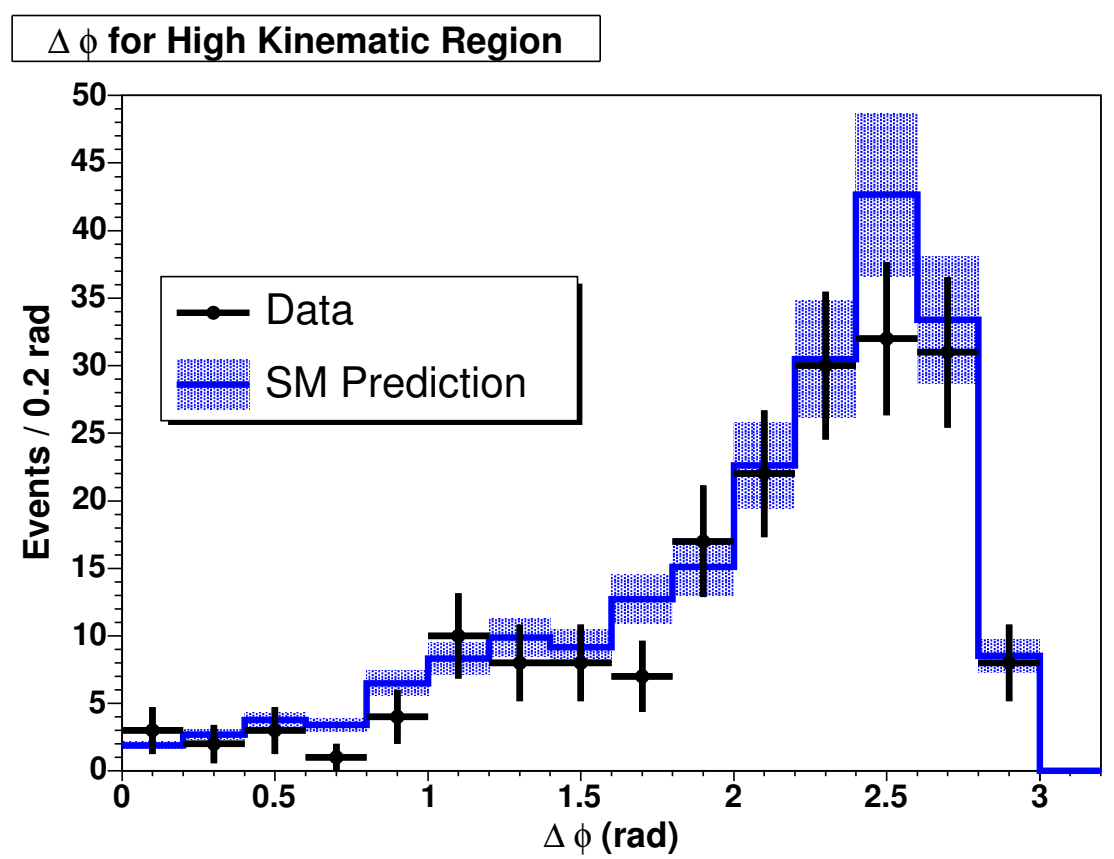

Figure 5.30: Comparison of the distribution for $\Delta \phi$ between the two jets for the 186 events in the high kinematic region candidate sample with the predicted SM distribution. 


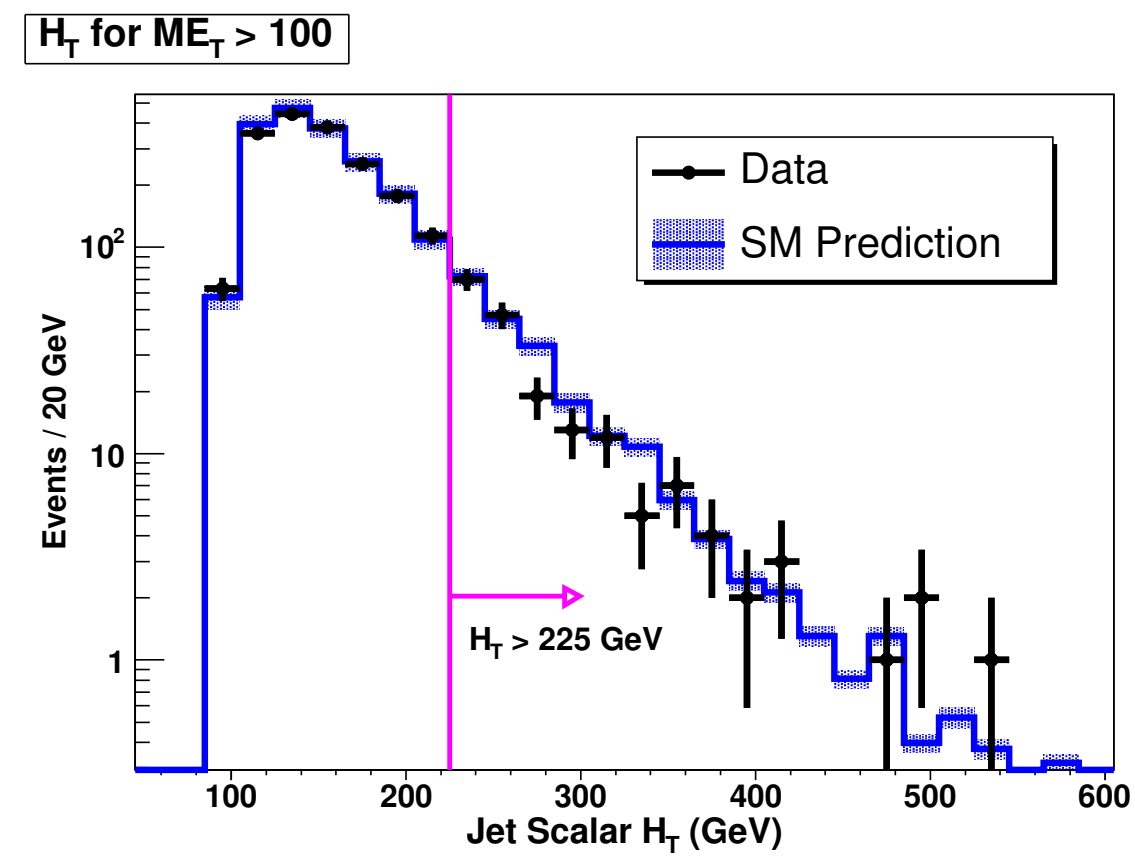

Figure 5.31: Comparison of the $H_{T}$ distribution between the SM prediction and data in the high kinematic region after removal of the jet $H_{T}$ selection cut. The Monte Carlo shape associated with each background process is normalized to its estimated contribution within the dijet plus missing $E_{T}$ signal region. The region to the right of the vertical line at $225 \mathrm{GeV}$ represents the high kinematic region. 


\section{Chapter 6}

\section{Leptoquarks}

Many possible extensions to the Standard Model could produce an excess number of events at $\mathrm{CDF}$ in the exclusive dijet $+\not_{T}$ signature. The results in Chapter 5 can be used to place limits on these models. In this chapter, a simple model of leptoquark pair production, with each leptoquark $L Q$ decaying to a quark $q$ and a neutrino $\nu$, is discussed.

\subsection{Leptoquark Models and Types}

Leptoquarks are strongly interacting particles which carry both lepton and baryon numbers. They are expected in a variety of different extensions to the Standard Model, such as SU(5) [45] or Pati-Salam SU(4) [46] unification, or models which postulate substructure for quarks and leptons [14]. Such particles can couple directly to both leptons and quarks, and can therefore decay to a lepton and a quark.

There are three restrictions made on the leptoquark model examined in this thesis, based on phenomenological constraints:

1. Only dimensionless couplings with the matter fields are considered, which keeps the theory renormalizable. 
2. Interaction terms are required to be $S U(3)_{C} \times S U(2)_{W} \times U(1)_{Y}$ gauge invariant (in other words, leptoquarks must interact like SM particles).

3. Only same generation couplings between lepton and quark are allowed.

The final restriction is based on existing experimental limits on flavour changing neutral current (FCNC) decays. As a result of this restriction, leptoquarks can be described as first, second, or third generation.

Overviews of the different possible types of leptoquarks appear in References [14] and [47]. Tables 6.1 and 6.2, adapted from [14] and [47], summarize the different types of leptoquarks. In these tables, $I_{W}$ represents the weak isospin of the leptoquark, $F$ is a quantity called the fermion number (defined as the lepton number $L$ plus 3 times the baryon number $B), Q$ is the electric charge, the couplings $\lambda_{L}$ and $\lambda_{R}$ denote coupling to left-handed or right-handed leptons, and $\beta_{e}$ is the fraction of leptoquarks which decay to an $e$ or $\bar{e}$ plus a quark.

In order to keep the interpretation of the analysis fairly general, three additional constraints are assumed which simplify the model under investigation. Firstly, only scalar leptoquark production is considered. This is because the production cross-section for scalar leptoquark pairs depends on only one free parameter: the leptoquark mass $M_{L Q}$. The cross-sections for the $q \bar{q} \rightarrow L Q \overline{L Q}$ and $g g \rightarrow L Q \overline{L Q}$ processes for scalar leptoquarks are

$$
\sigma(q \bar{q} \rightarrow L Q \overline{L Q})=\frac{2 \pi \alpha_{s}^{2}}{27 s} \beta^{3}
$$

and

$$
\sigma(g g \rightarrow L \overline{L Q Q})=\frac{\pi \alpha_{s}^{2}}{96 s}\left\{\beta\left(41-31 \beta^{2}\right)-\left(17-18 \beta^{2}+\beta^{4}\right) \ln \left|\frac{1+\beta}{1-\beta}\right|\right\}
$$

respectively. In these equations, $\alpha_{s}$ is the strong interaction coupling constant, $\sqrt{s}$ is the centre-of-mass energy for the parton-parton collision, and $\beta=\sqrt{1-4 M_{L Q}^{2} / s}$. The 


\begin{tabular}{|c|c|c|c|c|c|c|}
\hline Type & $I_{3 W}$ & $F=3 B+L$ & $Q$ & Decay & Coupling & $\beta_{e}$ \\
\hline$S_{1 L}$ & 0 & -2 & $-\frac{1}{3}$ & $e u, \nu_{e} d$ & $\lambda_{L},-\lambda_{L}$ & $\frac{1}{2}$ \\
\hline$S_{1 R}$ & 0 & -2 & $-\frac{1}{3}$ & $e u$ & $\lambda_{R}$ & $\frac{1}{2}$ \\
\hline$S_{1 R}$ & 0 & -2 & $-\frac{4}{3}$ & $e d$ & $\lambda_{R}$ & $\frac{1}{2}$ \\
\hline$S_{3 L}$ & 1 & -2 & $\begin{array}{l}-\frac{4}{3} \\
-\frac{1}{3} \\
\frac{2}{3}\end{array}$ & $\begin{array}{c}e d \\
e u, \nu_{e} d \\
\nu_{e} u\end{array}$ & $\begin{array}{c}-\sqrt{2} \lambda_{L} \\
\lambda_{L},-\lambda_{L} \\
\sqrt{2} \lambda_{L}\end{array}$ & $\begin{array}{l}1 \\
\frac{1}{2} \\
0\end{array}$ \\
\hline$R_{2 L}$ & $\frac{1}{2}$ & 0 & $\begin{array}{c}-\frac{5}{3} \\
\frac{2}{3}\end{array}$ & $\begin{array}{c}e u \\
\nu_{e} u\end{array}$ & $\begin{array}{l}\lambda_{L} \\
\lambda_{L}\end{array}$ & $\begin{array}{l}1 \\
0\end{array}$ \\
\hline$R_{2 R}$ & $\frac{1}{2}$ & 0 & $\begin{array}{r}-\frac{5}{3} \\
-\frac{2}{3} \\
\end{array}$ & $\begin{array}{l}e u \\
e d\end{array}$ & $\begin{array}{c}\lambda_{R} \\
-\lambda_{R}\end{array}$ & $\begin{array}{l}1 \\
1\end{array}$ \\
\hline$R_{2 L}$ & $\frac{1}{2}$ & 0 & $\begin{array}{c}-\frac{2}{3} \\
\frac{1}{3}\end{array}$ & $\begin{array}{c}e d \\
\nu_{e} \bar{d}\end{array}$ & $\begin{array}{l}\lambda_{L} \\
\lambda_{L}\end{array}$ & $\begin{array}{l}1 \\
0\end{array}$ \\
\hline
\end{tabular}

Table 6.1: Table of possible first-generation scalar (spin-0) leptoquark types. Second and third generation leptoquarks come in similar types, but with $s, c, \mu$ and $\nu_{\mu}$ or $b, t, \tau$ and $\nu_{\tau}$ replacing $d, u, e$ and $\nu_{e}$. 


\begin{tabular}{|c|c|c|c|c|c|c|}
\hline Type & $I_{3 W}$ & $F=3 B+L$ & $Q$ & Decay & Coupling & $\beta_{e}$ \\
\hline \hline$V_{2 L}$ & $\frac{1}{2}$ & -2 & $\begin{array}{r}-\frac{4}{3} \\
-\frac{1}{3}\end{array}$ & $\begin{array}{c}e d \\
\nu_{e} d\end{array}$ & $\lambda_{L}$ & 1 \\
& & & $-\frac{4}{3}$ & $e d$ & $\lambda_{R}$ & 1 \\
\hline$V_{2 R}$ & $\frac{1}{2}$ & -2 & $-\frac{1}{3}$ & $e u$ & $-\lambda_{R}$ & 1 \\
\hline$V_{2 L}$ & $\frac{1}{2}$ & -2 & $-\frac{1}{3}$ & $e u$ & $\lambda_{L}$ & 1 \\
& & & $\frac{2}{3}$ & $\nu_{e} u$ & $\lambda_{L}$ & 0 \\
\hline$U_{1 L}$ & 0 & 0 & $-\frac{2}{3}$ & $e d, \nu_{e} u$ & $\lambda_{L},-\lambda_{L}$ & $\frac{1}{2}$ \\
\hline$U_{1 R}$ & 0 & 0 & $-\frac{2}{3}$ & $e d$ & $\lambda_{R}$ & 1 \\
\hline$U_{1 R}$ & 0 & 0 & $-\frac{5}{3}$ & $e u$ & $\lambda_{R}$ & 1 \\
\hline$U_{3 L}$ & 1 & 0 & $-\frac{5}{3}$ & $e u$ & $-\sqrt{2} \lambda_{L}$ & 1 \\
& & & $-\frac{2}{3}$ & $e d, \nu_{e} u$ & $\lambda_{L},-\lambda_{L}$ & $\frac{1}{2}$ \\
& & & $\frac{1}{3}$ & $\nu_{e} \bar{d}$ & $\sqrt{2} \lambda_{L}$ & 0 \\
\hline
\end{tabular}

Table 6.2: Table of possible first-generation vector (spin-1) leptoquark types. Second and third generation leptoquarks come in similar types, but with $s, c, \mu$ and $\nu_{\mu}$ or $b, t, \tau$ and $\nu_{\tau}$ replacing $d, u, e$ and $\nu_{e}$. 
total leptoquark pair production cross-section at the Tevatron can be calculated by convoluting Equations 6.1 and 6.2 with the parton distribution functions for protons and anti-protons $[48,49]$. The pair production cross-sections for vector leptoquarks, however, are given by formulae which also involve model dependent parameters which describe possible trilinear $g L Q L Q$ or quartic $g g L Q L Q$ couplings. The cross-section formulae analogous to Equations 6.1 and 6.2 are given in full in Reference [49].

Two additional constraints are placed on the decay of the leptoquarks. The leptoquarks are assumed to decay before interacting with any other partons to form a hadronlike bound state, meaning the final state will come only from the decay products of the leptoquarks produced. The branching ratio for leptoquarks decaying to a quark and a neutrino is assumed to be one, meaning that $\beta_{e}=0$. Since the leptoquarks in this model have a single final state, the interpretation of the results is simplified.

These restrictions mean that the leptoquarks searched for in this interpretation are the ones in Table 6.1 with charge $\frac{1}{3}$ (which decay as $L Q \rightarrow v_{e} \bar{d}$ ) or $\frac{2}{3}$ (which decay as $\left.L Q \rightarrow v_{e} u\right)$. With $M_{L Q}$ the only free parameter in the model, this model provides a simple example interpretation of this analysis.

\subsection{Signal Monte Carlo}

To represent possible signals from leptoquark production in this analysis, Monte Carlo samples are generated using the PYTHIA event generator. These samples simulate pair production of leptoquarks, which decay only to a quark and a neutrino. Leptoquark samples are generated for masses between 50 and $200 \mathrm{GeV} / c^{2}$, in steps of $10 \mathrm{GeV} / c^{2}$. The processes $g g \rightarrow L Q \overline{L Q}$ and $q \bar{q} \rightarrow L Q \overline{L Q}$ are simulated. The leptoquark mass is directly set and the decays are restricted using the PYTHIA input parameters. By default, second generation leptoquarks are considered, with $L Q \rightarrow \bar{s} \nu_{\mu}$. First and third generation leptoquarks are also generated with masses of 150 and $180 \mathrm{GeV} / c^{2}$, with the 
decays $L Q \rightarrow \bar{d} \nu_{e}$ and $L Q \rightarrow \bar{b} \nu_{\tau}$, to examine the generational dependence on signal acceptance which comes from the differences between light and heavy quarks in the final state.

Instead of the leading order PYTHIA cross-sections, next-to-leading order (NLO) crosssections from Krämer et al. [50] are used. Note that the cross-sections used in previous searches for scalar leptoquarks at the Tevatron came from previous calculations by Krämer et al., found in Reference [48]. Table 6.3 shows the NLO cross sections for three different values of the renormalization scale. For this analysis, the renormalization scale $\mu=2 M_{L Q}$ is used, which gives the smallest production cross-sections, and therefore the most conservative limits.

\subsection{Previous Leptoquark Searches}

Searches for scalar leptoquark pairs decaying to $\nu \bar{\nu} q \bar{q}$ in the $E_{T}+$ dijet signature have been carried out previously at CDF. The previous best limits on first generation lepto-

quark masses came from a search carried out at CDF during Run II using $191 \mathrm{pb}^{-1}$ of data. These results ruled out the mass interval from 78 to $117 \mathrm{GeV} / \mathrm{c}^{2}$ at $95 \%$ C.L for first generation leptoquarks [51].

For higher generation leptoquarks, the CDF limits set in run I with $\sqrt{s}=1.8 \mathrm{GeV}$ and total integrated luminosity of $88 \mathrm{pb}^{-1}$ were higher than the limits set in Reference [51] because the Run I analysis used jet flavour identification to tag jets as containing a $c$ or $b$ quark, thus increasing the sensitivity to the decay of higher generation leptoquarks. The Run I 95\% C.L. lower mass limits for second and third generation scalar leptoquarks decaying to $\nu \bar{\nu} q \bar{q}$ were $123 \mathrm{GeV} / \mathrm{c}^{2}$ and $148 \mathrm{GeV} / \mathrm{c}^{2}$ respectively [52].

A dedicated search for third-generation leptoquarks has also been carried out at DØ during Run II of the Tevatron. As with the CDF search for third generation leptoquarks during Run I, this search used jet tagging methods to identify $b$ quark jets to increase 


\begin{tabular}{|c|c|c|c|}
\hline$M_{L Q}\left(\mathrm{GeV} / \mathrm{c}^{2}\right)$ & $\mu=\frac{1}{2} M_{L Q}(\mathrm{pb})$ & $\mu=M_{L Q}(\mathrm{pb})$ & $\mu=2 M_{L Q}(\mathrm{pb})$ \\
\hline 50 & 690. & 584. & 485. \\
\hline 60 & 270 . & 231. & 192. \\
\hline 70 & 119. & 103. & 86.4 \\
\hline 80 & 58.2 & 50.8 & 42.7 \\
\hline 90 & 30.6 & 26.9 & 22.7 \\
\hline 100 & 17.0 & 15.1 & 12.8 \\
\hline 110 & 9.96 & 8.91 & 7.59 \\
\hline 120 & 6.09 & 5.47 & 4.68 \\
\hline 130 & 3.85 & 3.47 & 2.97 \\
\hline 140 & 2.48 & 2.26 & 1.94 \\
\hline 150 & 1.66 & 1.51 & 1.30 \\
\hline 160 & 1.13 & 1.03 & 0.887 \\
\hline 170 & 0.781 & 0.713 & 0.614 \\
\hline 180 & 0.548 & 0.501 & 0.432 \\
\hline 190 & 0.389 & 0.356 & 0.307 \\
\hline 200 & 0.278 & 0.255 & 0.220 \\
\hline
\end{tabular}

Table 6.3: Calculated NLO cross sections for different values of the leptoquark mass and three different potential values of the renormalization scale, $\mu$. 
sensitivity to third generation leptoquarks. The results set a 95\% C.L. lower limit on the mass of the third generation scalar leptoquark of $229 \mathrm{GeV} / \mathrm{c}^{2}$ using $425 \mathrm{pb}^{-1}$ of data [53]. A D $\varnothing$ search using $310 \mathrm{pb}^{-1}$ of run II data searched for first and second generation scalar leptoquarks decaying to $\nu \bar{\nu} q \bar{q}$, setting a lower mass limit of $136 \mathrm{GeV} / \mathrm{c}^{2}$ [54].

Searches have also been carried out at CDF using the $l+E_{T}+\operatorname{dijet}$ and $l \bar{l}+$ dijet signatures, signatures which are optimized for leptoquarks with $\beta_{e}=0.5$ and 1 respectively. The results of these searches were combined with those from Reference [51] to set mass limits for first and second generation scalar leptoquarks as functions of $\beta_{e}$. For first generation scalar leptoquarks, the $95 \%$ C.L. lower limits on mass were $236 \mathrm{GeV} / \mathrm{c}^{2}$ for $\beta_{e}=1,205 \mathrm{GeV} / \mathrm{c}^{2}$ for $\beta_{e}=0.5$, and $145 \mathrm{GeV} / \mathrm{c}^{2}$ for $\beta_{e}=0.1$ [55]. For second generation scalar leptoquarks, the $95 \%$ C.L. lower limits on mass were $226 \mathrm{GeV} / \mathrm{c}^{2}$ for $\beta_{e}=1,208 \mathrm{GeV} / \mathrm{c}^{2}$ for $\beta_{e}=0.5$, and $143 \mathrm{GeV} / \mathrm{c}^{2}$ for $\beta_{e}=0.1[56]$.

At the HERA $e^{ \pm} p$ collider, leptoquarks may also have been singly produced through $e^{-} u, e^{-} d, e^{+} d$, or $e^{+} u \rightarrow L Q$ processes, with the decays $L Q \rightarrow e^{+} u, e^{+} d, \bar{\nu}_{e} u, e^{-} u, \nu_{e} d$ or $e^{-} d$ resulting in one or more jets with an electron, positron, or $\#_{T}$ in the final state. Searches for the production of single leptoquarks have been carried out by the ZEUS collaboration, which set limits on the mass of possible scalar or vector first-generation leptoquarks. These results are dependent on both $\beta_{e}$ and the magnitude of the coupling constant $\lambda$. The ZEUS analysis also does not search for leptoquarks which couple exclusively to quarks and neutrinos. At $\lambda=0.1$, ZEUS sets lower limits on $M_{L Q}$ ranging from 248 to $290 \mathrm{GeV} / c^{2}$, depending on the type of leptoquark [57]. 


\section{Chapter 7}

\section{Supersymmetry}

Another possible extension to the Standard Model which could produce an excess of events in the dijet $+E_{T}$ signature is supersymmetry (SUSY). In the Minimal Supersymmetric Extension to the Standard Model (MSSM), squarks $\tilde{q}$, the supersymmetric partners of the SM quarks, can decay to a jet-producing SM particle and a long-lived, stable, neutral SUSY particle which escapes the detector leading to $\not_{T}$. Pair production of these particles can thus result in large $\not_{T}$ accompanied by two energetic jets.

\subsection{The Minimal Supersymmetric Extension to the Standard Model}

\subsubsection{The Hierarchy Problem and Supermultiplets}

While theories involving leptoquarks connect the quark and lepton sectors of the SM, supersymmetric theories involve a symmetry between the fermion and boson sectors of the SM. One motivation for this comes from one-loop quantum corrections to the mass of the SM Higgs boson coming from its interactions with fermions and bosons. This problem is referred to as "the hierarchy problem."

If the Higgs couples to some fermion $f$ with a mass $m_{f}$ and a term in the Lagrangian 
of the form $\lambda_{f} H f \bar{f}$, the correction to the Higgs mass parameter $m_{H}^{2}$ becomes

$$
\Delta m_{H}^{2}=\frac{\left|\lambda_{f}\right|^{2}}{8 \pi^{2}} \Lambda_{U V}^{2}+\ldots
$$

where $\Lambda_{U V}$ is an ultraviolet momentum cutoff used to regulate the loop interval. $\Lambda_{U V}$ must be at least as large as the energy scale where new physics appears. The ellipsis represents terms proportional to $m_{f}^{2}$, and growing at most logarithmically with $\Lambda_{U V}$ [13]. If no new physics appears until the Planck scale $M_{P}=\left(8 \pi G_{\text {Newton }}\right)^{1 / 2}=2 \times 10^{18} \mathrm{GeV}$, this correction becomes some 30 orders of magnitude larger than the current Higgs limits. As mentioned in Section 1.2, these limits are $m_{H} \sim 100 \mathrm{GeV}[7,8,9]$.

A one-loop correction through coupling to a scalar boson $S$ with mass $m_{S}$ which appears in the Lagrangian with a term $-\lambda_{S}|H|^{2}|S|^{2}$ gives a one-loop quantum correction

$$
\Delta m_{H}^{2}=-\frac{\left|\lambda_{S}\right|^{2}}{16 \pi^{2}}\left[\Lambda_{U V}^{2}-2 m_{S}^{2} \ln \left(\Lambda_{U V} / m_{S}\right)+\ldots\right]
$$

Again, new physics at a large enough $\Lambda_{U V}$ scale would cause unreasonably large corrections in $m_{H}^{2}$. However, the relative minus sign between the fermion and boson corrections suggests that some sort of symmetry between the boson and fermion sectors could cause fermion mass corrections and those due to bosons to cancel. In fact, if each fermion were accompanied by a pair of complex scalar bosons (spin-0 particles represented by a field with real and imaginary components), and $\lambda_{S}=\left|\lambda_{f}\right|^{2}$, the $\Lambda_{U V}^{2}$ terms of equations 7.1 and 7.2 neatly cancel [13].

In supersymmetry, every particle in the SM forms a "supermultiplet" together with its supersymmetric partner or "superpartner." The SM interactions of particles in a given supermultiplet are set to be the same. In the simplest scenarios, each SM particle has a single superpartner. Each spin-1/2 fermion forms a supermultiplet with a spin-0 boson. These supermultiplets are called chiral supermultiplets, because there are separate supermultiplets for the left-handed and right-handed components of the fermion fields. Each spin-1 gauge boson is partnered with a spin-1/2 fermion in a supermultiplet. This 
configuration is called a gauge supermultiplet. If gravity is included, the spin-2 graviton $G$ has a spin-3/2 fermion superpartner $\tilde{G}$ called the gravitino. The Higgs boson's supermultiplets are slightly more complicated, so supersymmetry requires the presence of two Higgs supermultiplets. There are two charged and two neutral Higgs bosons, each forming chiral supermultiplets with their own superpartners [13].

Table 7.1 shows the chiral and gauge supermultiplets in the MSSM. In this table, the electroweak and Higgs bosons, the first generation quarks and leptons, and their superpartners are listed, along with their transformation properties under the SM gauge group $S U(3)_{C} \times S U(2)_{L} \times U(1)_{Y}$. The names follow the standard supersymmetry convention, where the superpartners of SM fermions are named using the "s-" prefix while those of bosons take an "-ino" suffix. Squarks and sleptons are collectively called "sfermions", while the superpartners of the gauge bosons are called "gauginos". The superpartners of SM particles are known as "sparticles", which is short for "supersymmetric particles."

The left-handed and right-handed components of the quarks and leptons appear separately in Table 7.1, as they have different gauge transformations in the SM. This means that the the left and right-handed components have separate superpartners, denoted with " $L$ " and " $R$ " subscripts. This introduces two complex scalar fields for each SM Dirac fermion, thus meeting the requirements for cancellation of the $\Lambda_{U V}^{2}$ terms in equations 7.1 and 7.2. Of course, being spin-0 particles, " $L$ " and " $R$ " does not refer the helicity of the superpartners, but to the helicity of the SM fermion they are associated with. However, since the gauge interactions for SM particles and their superpartners are exactly the

same, only the "left-handed" sfermions $\tilde{e}_{L}, \tilde{\mu}_{L}, \tilde{\tau}_{L}, \tilde{u}_{L}, \tilde{d}_{L}, \tilde{c}_{L}, \tilde{s}_{L}, \tilde{b}_{L}$, and $\tilde{t}_{L}$ couple to the $W$ boson [13].

\subsubsection{R-parity}

One important property introduced in the MSSM is R-parity, $P_{R}$, defined as 


\begin{tabular}{|c|c|c|c|c|}
\hline \multicolumn{2}{|l|}{ Particles } & spin 0 boson & spin $1 / 2$ fermion & $S U(3)_{C}, S U(2)_{L}, U(1)_{Y}$ \\
\hline squarks and quarks & $Q$ & $\left(\tilde{u}_{L}, \tilde{d}_{L}\right)$ & $\left(u_{L}, d_{L}\right)$ & $\left(3,2, \frac{1}{6}\right)$ \\
\hline ( $\times 3$ families $)$ & $\bar{u}$ & $\left(\tilde{u}_{R}^{*}\right)$ & $\left(u_{R}^{\dagger}\right)$ & $\left(\overline{3}, 1,-\frac{2}{3}\right)$ \\
\hline & $\bar{d}$ & $\left(\tilde{d}_{R}^{*}\right)$ & $\left(d_{R}^{\dagger}\right)$ & $\left(\overline{3}, 1, \frac{1}{3}\right)$ \\
\hline sleptons and leptons & $L$ & $\left(\tilde{\nu}, \tilde{e}_{L}\right)$ & $\left(\nu, e_{L}\right)$ & $\left(1,2,-\frac{1}{2}\right)$ \\
\hline$(\times 3$ families $)$ & $\bar{e}$ & $\left(\tilde{e}_{R}^{*}\right)$ & $\left(e_{R}^{\dagger}\right)$ & $(1,1,1)$ \\
\hline Higgs and higgsinos & $H_{u}$ & $H_{u}^{+}, H_{u}^{0}$ & $\tilde{H}_{u}^{+}, \tilde{H}_{u}^{0}$ & $\left(1,2, \frac{1}{2}\right)$ \\
\hline & $H_{d}$ & $H_{d}^{0}, H_{d}^{-}$ & $\tilde{H}_{d}^{0}, \tilde{H}_{d}^{-}$ & $\left(1,2,-\frac{1}{2}\right)$ \\
\hline \multicolumn{2}{|l|}{ Particles } & spin 1 boson & spin $1 / 2$ fermion & $S U(3)_{C}, S U(2)_{L}, U(1)_{Y}$ \\
\hline \multicolumn{2}{|l|}{ gluon and gluino } & $g$ & $\tilde{g}$ & $(8,1,0)$ \\
\hline \multicolumn{2}{|l|}{$W$ bosons and winos } & $W^{ \pm}, W^{0}$ & $\tilde{W}^{ \pm}, \tilde{W}^{0}$ & $(1,3,0)$ \\
\hline \multicolumn{2}{|l|}{$B$ boson and bino } & $B^{0}$ & $\tilde{B}^{0}$ & $(1,1,0)$ \\
\hline
\end{tabular}

Table 7.1: Chiral and gauge supermultiplets of the MSSM, after [13]. The asterisk superscript represents the complex conjugate of a field, and the dagger represents its Hermitian conjugate. Only the first generation SM fermions and sfermions are shown. 


$$
P_{R}=(-1)^{3(B-L)+2 s}
$$

where $B$ is the baryon number carried by the particle, $L$ is the particle's lepton number, and $s$ is its spin. All SM particles, including the MSSM's four Higgs bosons, have even R-parity $\left(P_{R}=+1\right)$, while all squarks, sleptons, higgsinos, and gauginos have odd Rparity $\left(P_{R}=-1\right)$. If $\mathrm{R}$-parity is conserved, this leads to three major consequences for SUSY phenomenology:

- The lightest particle with $P_{R}=-1$ must be stable. This particle is referred to as the Lightest Supersymmetric Particle (LSP).

- Every other supersymmetric particle must decay into a state with an odd number of LSPs (usually one.)

- Since collisions in collider experiments start with the interaction of two R-parity even SM particles, supersymmetric particles must be produced in even numbers (usually in pairs.)

If the LSP also has no electric or colour charge and thus interacts only weakly with ordinary baryonic matter, it may be a WIMP dark matter candidate (see Section 1.2.) R-parity conservation also leads naturally to conservation of baryon and lepton number, agreeing with current experimental bounds on their non-conservation. In most discussions of the MSSM, R-parity is assumed to be conserved [13].

\subsubsection{Mass Eigenstates}

The superparticles in Table 7.1 are presented in terms of their eigenstates under gauge transformations. Each row describes a supermultiplet containing particles with identical $S U(3)_{C} \times S U(2)_{L} \times U(1)_{Y}$ quantum numbers. However, after the effects of electroweak symmetry and supersymmetry breaking, the electroweak gauginos and higgsinos can 
mix, as can the squarks, leptons, and Higgs scalar bosons with the same charge. For the squarks and sleptons, mixing of gauge eigenstates is highest for the third generation due to the larger fermion masses involved. The term in the MSSM Lagrangian corresponding to the mass of the stop squarks is given by

$$
\mathcal{L}_{\text {stop masses }}=-\left(\tilde{t}_{L}^{*} \tilde{t}_{R}^{*}\right) \mathbf{m}_{\tilde{t}}^{2}\left(\begin{array}{c}
\tilde{t}_{L}^{*} \\
\tilde{t}_{R}^{*}
\end{array}\right)
$$

For the stop squarks, the off-diagonal elements of the mass matrix $\mathbf{m}_{\tilde{t}}^{2}$ are proportional to the mass of the top quark. The off-diagonal elements in the analogous matrices for the other sfermions similarly depend on the masses of the fermions. When the mass matrices are diagonalized to find the mass eigenstates, the mixing angle becomes large for third-generation particles due to these large off-diagonal elements. By contrast, the first two generations have relatively small fermion masses, leading to very small mixing angles and thus negligible mixing [13]. The mass eigenstates for the unobserved MSSM particles, assuming sfermion mixing for the first two generations is small, are given in Table 7.2.

\subsubsection{Supersymmetry Models}

In addition to the identical gauge couplings, unbroken supersymmetry would mean that SM particles and their superpartners would have the same mass. Obviously, if supersymmetry exists it must be a broken symmetry, since only their SM partners have been seen experimentally ${ }^{1}$. If broken supersymmetry is still supposed to solve the hierarchy problem, the effective Lagrangian must take the form

$$
\mathcal{L}=\mathcal{L}_{\mathrm{SUSY}}+\mathcal{L}_{\mathrm{soft}}
$$

\footnotetext{
${ }^{1}$ Conversely, supersymmetry has been half-seriously described as a very successful theory, since nearly half of the particles postulated have been discovered.
} 


\begin{tabular}{|c|c|c|c|c|}
\hline Names & Spin & $P_{R}$ & Gauge eigenstates & Mass eigenstates \\
\hline \hline Higgs bosons & 0 & +1 & $H_{u}^{0}, H_{d}^{0}, H_{u}^{+}, H_{d}^{-}$ & $h^{0}, H^{0}, A^{0}, H^{ \pm}$ \\
1st gen. squarks & 0 & -1 & $\tilde{u}_{L}, \tilde{u}_{R}, \tilde{d}_{L}, \tilde{d}_{R}$ & (same) \\
2nd gen. squarks & 0 & -1 & $\tilde{s}_{L}, \tilde{s}_{R}, \tilde{c}_{L}, \tilde{c}_{R}$ & (same) \\
3rd gen. squarks & 0 & -1 & $\tilde{t}_{L}, \tilde{t}_{R}, \tilde{b}_{L}, \tilde{b}_{R}$ & $\tilde{t}_{1}, \tilde{t}_{2}, \tilde{b}_{1}, \tilde{b}_{2}$ \\
\hline 1st gen. sleptons & 0 & -1 & $\tilde{e}_{L}, \tilde{e}_{R}, \tilde{\nu}_{e}$ & $($ same) \\
2nd gen. sleptons & 0 & -1 & $\tilde{\mu}_{L}, \tilde{\mu}_{R}, \tilde{\nu}_{\mu}$ & $($ same $)$ \\
3rd gen. sleptons & 0 & -1 & $\tilde{\tau}_{L}, \tilde{\tau}_{R}, \tilde{\nu}_{\tau}$ & $\tilde{\tau}_{1}, \tilde{\tau}_{2}, \tilde{\nu}_{\tau}$ \\
\hline neutralinos & $1 / 2$ & -1 & $\tilde{B}^{0}, \tilde{W}^{0}, \tilde{H}_{u}^{0}, \tilde{H}_{d}^{0}$ & $\tilde{\chi}_{1}^{0}, \tilde{\chi}_{2}^{0}, \tilde{\chi}_{3}^{0}, \tilde{\chi}_{4}^{0}$ \\
charginos & $1 / 2$ & -1 & $\tilde{W}^{ \pm}, \tilde{H}_{u}^{+}, \tilde{H}_{d}^{-}$ & $\tilde{\chi}_{1}^{ \pm}, \tilde{\chi}_{2}^{ \pm}$ \\
gluino & $1 / 2$ & -1 & $\tilde{g}_{(\text {same })}$ \\
\hline \hline
\end{tabular}

Table 7.2: Mass and gauge eigenstates of the MSSM, after [13].

where $\mathcal{L}_{\text {SUSY }}$ has all of the gauge and Yukawa interactions and keeps supersymmetry invariance, while all the SUSY-breaking terms are in $\mathcal{L}_{\text {soft }} \cdot \mathcal{L}_{\text {soft }}$ only has mass terms and coupling parameters with positive mass dimension, and involves only the scalar and gaugino particles. This prevents quadratically divergent radiative corrections to the Higgs mass from appearing $[13,58]$. In terms of $m_{\text {soft }}$, the largest mass scale associated with $\mathcal{L}_{\text {soft }}$, the corrections to the Higgs mass parameter are of the form

$$
\Delta m_{H}^{2}=m_{\mathrm{soft}}\left[\frac{\lambda}{16 \pi^{2}} \ln \left(\Lambda_{U V} / m_{\mathrm{soft}}\right)+\ldots\right]
$$

with $\lambda$ being a dimensionless coupling, and vanish as $m_{\text {soft }} \rightarrow 0$. If $\lambda=1, \Lambda_{U V}=M_{P}$, and the masses of the Higgs bosons are presumed to be small enough to give the observed $W$ and $Z$ masses, $m_{\text {soft }}$ is around $1 \mathrm{TeV}$ at most. Since the masses of the sparticles should be close to $m_{\text {soft }}$, this may put them in a mass range which would be visible at the Tevatron [13]. 
The theoretical question of interest when defining a particular model within an MSSM framework is how SUSY is broken. A SUSY-breaking mechanism should also help organize or eliminate the 105 free parameters which appear in the most general $\mathcal{L}_{\text {soft }}$ which describes spontaneous soft supersymmetry breaking [13]. Some of the more popular models are minimal supergravity (mSUGRA) models, where supersymmetry is broken by gravitational-strength interactions near the Planck scale. These mSUGRA models reduce the number of free parameters to five. This is done through constructing a $\mathcal{L}_{\text {soft }}$ consistent with Planck scale SUSY breaking, and simplifying it by assuming the scalar masses and couplings are flavour-diagonal and universal at the Planck scale. Other possible SUSY-breaking mechanisms which include gauge-mediated SUSY breaking (GMSB) theories, where gauge interactions break SUSY via interactions with additional supermultiplets of massive particles, and SUSY breaking due to extra spatial dimensions [13].

The MSSM model used to interpret the results of this analysis is inspired in part by the results of a previous study in an inclusive jets $+E_{T}$ signature carried out in CDF's Run I [59]. This is a very general model, which makes no assumptions about the mechanism of supersymmetry breaking, and uses the most general set of soft supersymmetry breaking terms allowed by the symmetries. In this model:

- The lightest neutralino $\tilde{\chi}_{1}^{0}$ is also the lightest supersymmetric particle.

- The four squarks $\tilde{q}=\tilde{u}, \tilde{d}, \tilde{c}, \tilde{s}$ in the first two families are degenerate in mass.

- The gluino $\tilde{g}$ is more than $20 \%$ heavier than the squark.

There are several important consequences of these constraints. Firstly, no mSUGRA solutions exist for these choices of squark and gluino mass, and GMSB theories have the gravitino $\tilde{G}$ as the LSP, so the MSSM model examined here does not fit into either category. $^{2}$ Secondly, since squarks decay primarily to one quark and one neutralino,

\footnotetext{
${ }^{2}$ Some GMSB models have a long-lived $\tilde{\chi}_{1}^{0}$ being the next-to-lightest sparticle (NLSP), and decaying slowly into $\gamma+\tilde{G}$. GMSB models where the $\tilde{\chi}_{1}^{0}$ lifetime is long enough that it decays outside the detector are phenomenologically indistinguishable from the MSSM used here.
} 
while gluinos generally undergo a decay chain ending in a final state containing two or more jets and a neutralino [13], exclusive dijet + missing $E_{T}$ final states are expected to dominate. This is in contrast to the run II searches using a multijet plus missing $E_{T}$ signature which are interpreted using minimal supergravity mSUGRA scenarios [60, 61]. Reference [59] used an mSUGRA model in the $m_{\tilde{q}}>m_{\tilde{g}}$ region, and a non-mSUGRA MSSM model similar to the one used in this analysis in the $m_{\tilde{q}}>m_{\tilde{g}}$ region.

The MSSM model used in this analysis is characterized by several parameters. The main ones are the masses of the sparticles of interest. These particle masses are changed to produce several different mass spectra, as discussed in Section 7.2. The model assumes that the squarks from the first and second generation have the same mass $m_{\tilde{q}}$, and ignores the third generation squarks in order to avoid having to consider mixing effects. Sleptons are also not considered in this analysis. Likewise, since the gravitino does not enter into the phenomenology of this non-GMSB model, it is ignored. Three other parameters remain the same for all the mass spectra in this analysis. They are the ratio of the Higgs vacuum expectation values at the electroweak scale $(\tan \beta)$, the third generation trilinear coupling $\left(A_{T}\right)$, and the Higgsino mixing parameter $(\mu$.)

\subsubsection{Squark and gluino production}

Since R-parity is conserved in this model, squarks and gluinos will be produced in pairs at the Tevatron. As with their SM partners, they can be produced in $p \bar{p}$ collisions through gluon-gluon, quark-quark, or quark-antiquark interactions.

The squark pair production processes are:

$$
\begin{aligned}
& q_{i}+q_{j} \rightarrow \tilde{q}_{i L}+\tilde{q}_{j L} \\
& q_{i}+q_{j} \rightarrow \tilde{q}_{i R}+\tilde{q}_{j R} \\
& q_{i}+q_{j} \rightarrow \tilde{q}_{i L}+\tilde{q}_{j R}+
\end{aligned}
$$




$$
\begin{aligned}
q_{i}+\bar{q}_{j} & \rightarrow \tilde{q}_{i L}+\overline{\tilde{q}}_{j L} \\
q_{i}+\bar{q}_{j} & \rightarrow \tilde{q}_{i R}+\overline{\tilde{q}}_{j R} \\
q_{i}+\bar{q}_{j} & \rightarrow \tilde{q}_{i L}+\overline{\tilde{q}}_{j R}+ \\
q_{i}+\bar{q}_{i} & \rightarrow \tilde{q}_{j L}+\overline{\tilde{q}}_{j L} \\
q_{i}+\bar{q}_{i} & \rightarrow \tilde{q}_{j R}+\overline{\tilde{q}}_{j R} \\
g+g & \rightarrow \tilde{q}_{i L}+\overline{\tilde{q}}_{i L} \\
g+g & \rightarrow \tilde{q}_{i R}+\overline{\tilde{q}}_{i R}
\end{aligned}
$$

where a trailing "+" indicates that charge-conjugate modes are included. The gluino pair production processes are:

$$
\begin{aligned}
q_{i}+\bar{q}_{i} & \rightarrow \tilde{g}+\tilde{g} \\
g+g & \rightarrow \tilde{g}+\tilde{g}
\end{aligned}
$$

The squark + gluino production processes are:

$$
\begin{aligned}
& q_{i}+g \rightarrow \tilde{q}_{i L}+\tilde{g} \\
& q_{i}+g \rightarrow \tilde{q}_{i R}+\tilde{g}
\end{aligned}
$$

\subsubsection{Squark and gluino decay}

In theories where $m_{\tilde{q}}>m_{\tilde{g}}$, the decay $\tilde{q} \rightarrow q \tilde{g}$ is dominant. This is not the case here, meaning that the dominant decays are

$$
\begin{aligned}
& \tilde{q} \rightarrow \tilde{\chi}_{i}^{0}+q \\
& \tilde{q} \rightarrow \tilde{\chi}_{i}^{ \pm}+q^{\prime}
\end{aligned}
$$


Since the lightest neutralino is the LSP, the decay $\tilde{q} \rightarrow q+\tilde{\chi}_{1}^{0}$ will be kinematically favoured. For the "right-handed squarks" $\tilde{q}_{R}$, this is expected to be the predominant decay because the mass eigenstate $\tilde{\chi}_{1}^{0}$ is mostly made up of the gauge eigenstate $\tilde{B}$. Other charginos and neutralinos contain the wino gauge eigenstates, to which the righthanded squarks do not couple [13]. If a pair of $\tilde{q}_{R}$ are produced and R-parity is conserved, this leads to two quarks and two neutralinos, and thus to two jets and $E_{T}$.

The decay products of left-handed squarks, when they do not decay directly to $\tilde{q} \rightarrow$ $q+\tilde{\chi}_{1}^{0}$, depend on the decays of the charginos and heavier neutralinos. This may result in the production of additional jets or leptons, since the charginos and heavier neutralinos will undergo a series of decays. These decays include:

$$
\begin{aligned}
& \tilde{\chi}_{i}^{0} \rightarrow Z+\tilde{\chi}_{j}^{0}, W+\tilde{\chi}_{j}^{ \pm}, h^{0}+\tilde{\chi}_{j}^{0} \\
& \tilde{\chi}_{i}^{ \pm} \rightarrow Z+\tilde{\chi}_{j}^{ \pm}, W+\tilde{\chi}_{j}^{0}, h^{0}+\tilde{\chi}_{j}^{ \pm}
\end{aligned}
$$

Gluinos can only decay through processes involving a real or virtual squark. In this model, since $m_{\tilde{q}}<m_{\tilde{g}}$, they will decay primarily through $\tilde{g} \rightarrow q \tilde{q}$. The squarks decay as previously discussed. This leads to more than one jet, and possibly leptons, in the final state in addition to the $\mathscr{E}_{T}$ from the escaping $\tilde{\chi}_{1}^{0}$. In models where $m_{\tilde{q}}>m_{\tilde{g}}$, the gluino can only decay through three-body decay processes involving an off-shell squark, such as $\tilde{g} \rightarrow q \tilde{q}^{*} \rightarrow q q \tilde{\chi}_{1}^{0}[13]$

\subsection{Signal Monte Carlo}

The masses of the supersymmetric particles are used as input for the MSSM model in this analysis. The chosen values for the other MSSM parameters are the same as those used in the Run I analysis: $\tan \beta=3.0, A_{T}=-500$, and $\mu=-800$. Four different mass spectra are used for this analysis. Two of the mass spectra, denoted S2 and S4, were previously investigated in the Run I analysis, but not ruled out. Two more points (S1 and S3) on 


\begin{tabular}{|c|c|c|c|c|c|}
\hline Spectrum & $M_{\tilde{q}}\left(\mathrm{GeV} / c^{2}\right)$ & $M_{\tilde{g}}\left(\mathrm{GeV} / c^{2}\right)$ & $M_{\tilde{\chi}_{1}^{0}}\left(\mathrm{GeV} / c^{2}\right)$ & \multicolumn{2}{|c|}{$\sigma_{L O}(\mathrm{pb})$} \\
& & & & $\tilde{q}_{R}$ & Inclusive \\
\hline \hline $\mathrm{S} 1$ & 320 & 390 & 60 & 0.045 & 0.36 \\
\hline $\mathrm{S} 2$ & 250 & 450 & 72 & 0.28 & 1.73 \\
\hline $\mathrm{S} 3$ & 220 & 520 & 85 & 0.61 & 3.21 \\
\hline S4 & 120 & 550 & 89 & 18.0 & 57.4 \\
\hline
\end{tabular}

Table 7.3: MSSM particle masses examined in this analysis. Cross-sections come from leading order PYTHIA cross-sections.

the $m_{\tilde{q}}$ vs. $m_{\tilde{g}}$ plot lying in the region not ruled out in run I and with no mSUGRA solution possible are chosen. ISAJET [62] is then used for those masses to determine a $\tilde{\chi}_{1}^{0}$ mass in a generic MSSM scenario. The squark, gluino, and neutralino masses for the four spectra examined are given in Table 7.3. Figure 7.1 compares the squark and gluino masses for these spectra to masses excluded by previous searches [59, 63].

PYTHIA is used to generate two samples for each mass spectrum. The first sample contains exclusively pair production of right-handed squarks. The second sample contains all processes which produce a pair of squarks, a pair of gluinos, or a squark-gluino pair. This sample is called the inclusive sample. The LO cross-section calculated by PYTHIA is also found for all eight MC samples, in both the inclusive and the RH-only cases.

While the inclusive sample has a larger total cross-section than the RH-only sample, the gluinos and left-handed squarks are more likely to be rejected by the cuts in this analysis. This is because the neutralinos, $\tilde{\chi}_{i}^{0}$, are mass eigenstates of the neutral Higgsinos $\tilde{H}_{u, d}^{0}$, bino $\tilde{B}^{0}$ and neutral wino $\tilde{W}^{0}$. The $\tilde{B}^{0}$ and $\tilde{W}^{0}$ are supersymmetric partners of the $B^{0}$ and $W^{0}$ bosons which mix after electroweak symmetry breaking to produce the $Z^{0}$ and $\gamma$. The $\tilde{\chi}_{1}^{0}$ is mostly $\tilde{B}^{0}$ in the model examined. The $\tilde{\chi}_{i}^{ \pm}$are mass eigenstates of the charged Higgsinos $\tilde{H}_{u, d}^{ \pm}$and charged winos $\tilde{W}^{ \pm}$. With $\tilde{\chi}_{1}^{0}$ being the lightest supersymmetric particle, the $\tilde{q} \rightarrow q \tilde{\chi}_{1}^{0}$ is kinematically possible for left-handed 
and right-handed squarks. However, due to the large $\tilde{q} q \tilde{W}^{ \pm}$couplings, which are not present for right-handed squarks, left-handed squarks are likely to go through the decays $\tilde{q} \rightarrow q^{\prime} \tilde{\chi}_{i}^{ \pm}$and $\tilde{q} \rightarrow q^{\prime} \tilde{\chi}_{i \neq 1}^{0}$. The charginos and heavier neutralinos produced can decay producing leptons, causing the event to be rejected by the isolated track veto. Gluinos decay predominantly as $\tilde{g} \rightarrow q \tilde{q}$, producing an extra jet in the event [13]. This means events with gluinos are also less likely to pass the dijet cuts. Thus, the inclusive samples have generally lower acceptances. Spectrum S4 is an exception, where the acceptances in both cases are low, but the inclusive acceptance is slightly higher.

\subsection{Previous squark/gluino searches at the Tevatron}

A Run I CDF analysis [59] examined $84.37 \mathrm{pb}^{-1}$ of $1.8 \mathrm{TeV} p \bar{p}$ collisions, searching for events with three or more high- $E_{T}$ jets and $E_{T}$. These results appear in green in Figure 7.1 .

Recent analyses at Run II have also searched for squarks and gluinos in a multijet $+E_{T}$ signature. The latest results at CDF [60], using $2.0 \mathrm{fb}^{-1}$ of data, confine the interpretation of their results to mSUGRA models. In their models, the squarks decay predominantly to gluinos, resulting in a higher jet multiplicity. For the CDF multijet + $\not_{T}$ analysis, three different sets of cuts were used: one for two or more jets, one for three

or more jets, and one for four or more jets. A similar analysis using $2.1 \mathrm{fb}^{-1}$ of data from the $\mathrm{D} \varnothing$ experiment [61] also analysed two, three, and four jets plus $\not_{T}$ signatures separately. 


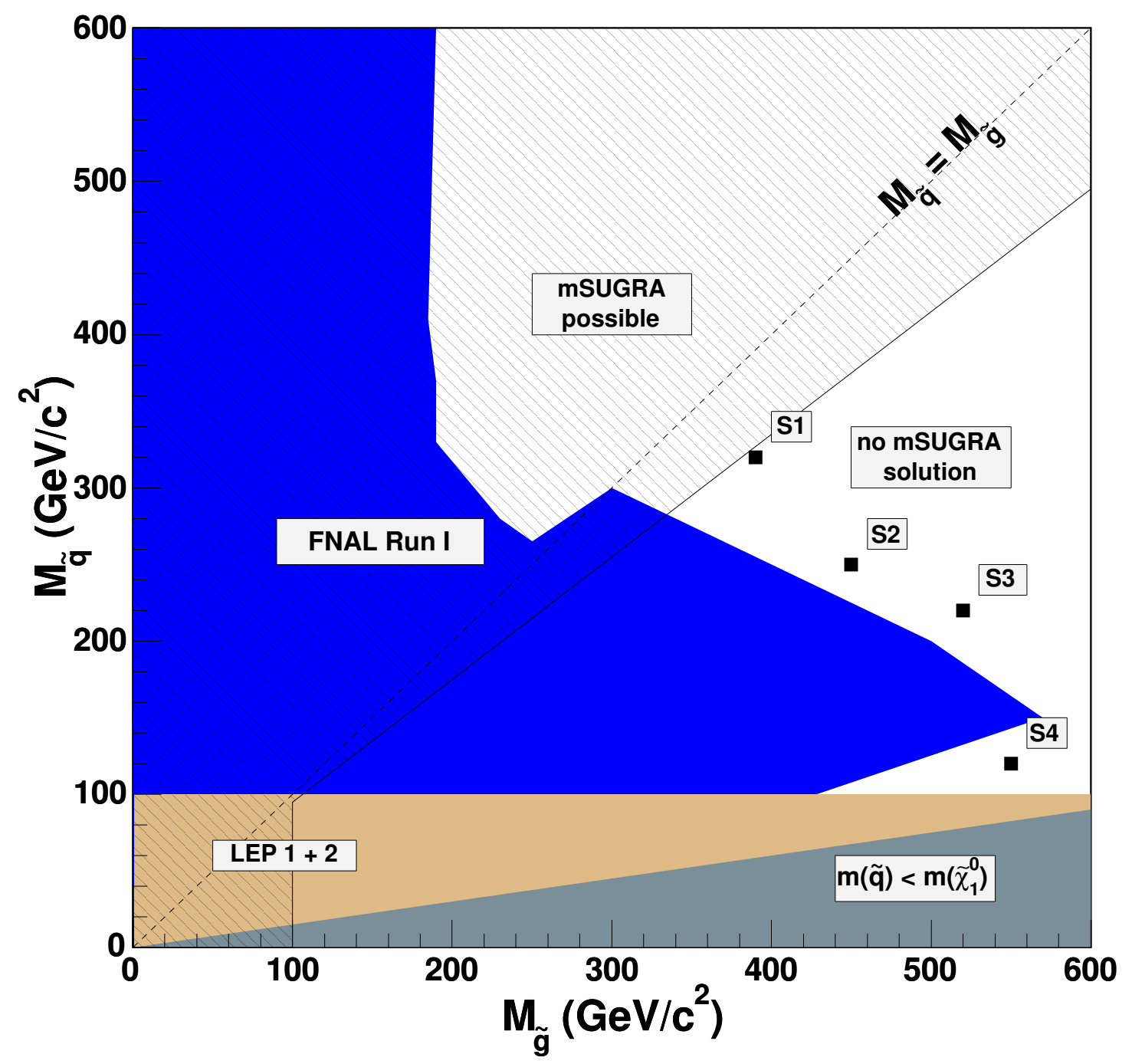

Figure 7.1: Squark and gluino masses ruled out by previous searches [59, 63] compared to spectra examined in this analysis. 


\section{Chapter 8}

\section{Analysis and Results}

\subsection{Results from Data and Limit Calculations}

As shown in Table 5.10, the result for the total background prediction is $2443 \pm 145$ events for the low kinematic region. With 2506 events found in the data, this corresponds to a 0.43 standard deviation excess in the data compared to the SM prediction. In the high kinematic region, $211 \pm 30$ events were predicted, and 186 events were found. In other words, the data is 0.34 standard deviations below the SM expectations. In both kinematic regions, the results are within 0.5 standard deviations of the predictions. Since the discrepancies are small, no discovery of new physics is possible. However, these results can be used to place limits on possible new physics.

The number of observed events, the predicted number of background events, and the uncertainty on the background prediction can be used to determine $95 \%$ confidence level (CL) upper limits on the number of signal events from new physics. A non-SM model with a production cross-section times event acceptance $\left(A_{s i g}\right)$ resulting in a signal contribution above this limit, given the integrated luminosity of the data sample used, can in general be ruled out. This limit also depends on the uncertainty $\Delta A_{s i g}$ on the $A_{s i g}$ for a particular model. 


\begin{tabular}{|l|c|c|}
\hline$\Delta A_{\text {sig }}(\%)$ & A priori Upper Limit & Observed Upper Limit \\
\hline \hline $7.5 \%$ & 298 & 346 \\
\hline $10.0 \%$ & 302 & 351 \\
\hline $12.5 \%$ & 307 & 357 \\
\hline $15.0 \%$ & 314 & 365 \\
\hline $17.5 \%$ & 321 & 375 \\
\hline $20.0 \%$ & 332 & 386 \\
\hline $22.5 \%$ & 343 & 400 \\
\hline $25.0 \%$ & 356 & 417 \\
\hline $27.5 \%$ & 372 & 436 \\
\hline $30.0 \%$ & 391 & 459 \\
\hline $32.5 \%$ & 413 & 486 \\
\hline
\end{tabular}

Table 8.1: A priori and observed 95\% CL upper limits on the number of non-SM signal events contributing to the low kinematic region candidate sample as a function of signal acceptance uncertainty.

Tables 8.1 and 8.2 present these event limits for the low and high regions respectively in terms of $\Delta A_{\text {sig. }}$. The upper limits are calculated using a Bayesian approach with a flat prior for the number of signal events, and gamma distributions for the priors for the acceptance and the number of signal events [64]. The a priori limits quoted are the limits which would have been obtained if the number of events in data had agreed exactly with the total background predictions. Since two kinematic regions are independently tested, the final limits in each model examined come from the kinematic region where the a priori result leads to the most sensitive cross-section $95 \%$ CL limit.

Section 8.2 discusses the general methods used for $\Delta A$ calculation. Section 8.3 applies these methods to scalar leptoquark pair production, and uses the results to interpret this analysis in terms of production cross-section upper limits and mass lower limits for 


\begin{tabular}{|l|c|c|}
\hline$\Delta A_{\text {sig }}(\%)$ & A priori Upper Limit & Observed Upper Limit \\
\hline \hline $7.5 \%$ & 63 & 46 \\
\hline $10.0 \%$ & 64 & 47 \\
\hline $12.5 \%$ & 65 & 48 \\
\hline $15.0 \%$ & 67 & 49 \\
\hline $17.5 \%$ & 68 & 50 \\
\hline $20.0 \%$ & 70 & 51 \\
\hline $22.5 \%$ & 73 & 53 \\
\hline $25.0 \%$ & 76 & 55 \\
\hline $27.5 \%$ & 79 & 57 \\
\hline $30.0 \%$ & 83 & 60 \\
\hline $32.5 \%$ & 88 & 63 \\
\hline
\end{tabular}

Table 8.2: A priori and observed 95\% CL upper limits on the number of non-SM signal events contributing to the high kinematic region candidate sample as a function of signal acceptance uncertainty. 
scalar leptoquarks. Section 8.4 applies these methods to four sample MSSM spectra, and compares the cross-section upper limits to those determined by PYTHIA simulation.

\subsection{Uncertainties on Signal Acceptance}

The uncertainty on signal acceptance for a model of new physics in this analysis combines six sources of uncertainty: the choice of parton distribution function, the jet energy scale, the combined effects of initial and final state radiation, the uncertainty on the luminosity measurement, the statistical error from the Monte Carlo samples used, and the efficiency of the clean-up cuts. These sources of uncertainty are considered to be independent of one another, and are therefore added in quadrature to find the total uncertainty $\Delta A_{\text {sig }}$. The same methods were used in the leptoquark and MSSM interpretations of this analysis.

\subsubsection{Choice of Parton Distribution Function}

By default, the Monte Carlo used in this analysis uses the CTEQ5L parton distribution function (PDF) [65]. To determine the uncertainty on acceptance due to choice of PDF, CTEQ6 [66] and MRST [67] PDF sets are used for comparisons. The process used is that prescribed by the CDF Joint Physics group [70].

Since all events in the signal Monte Carlo initially come from $p \bar{p}$ interactions, the incoming parton (quark, antiquark, or gluon) from the $p$ and the $\bar{p}$ can be identified. Each event is then re-weighted according to the formula

$$
w\left(x_{p}, x_{\bar{p}}, Q\right)=\frac{f_{p}^{A}\left(x_{p}, Q\right) \cdot f_{\bar{p}}^{A}\left(x_{\bar{p}}, Q\right)}{f_{p}^{B}\left(x_{p}, Q\right) \cdot f_{\bar{p}}^{B}\left(x_{\bar{p}}, Q\right)}
$$

where $w\left(x_{p}, x_{\bar{p}}, Q\right)$ is the new weight in $\mathrm{PDF} A$ for an event originally generated with $\mathrm{PDF} B, f_{p}^{P}\left(x_{p}, Q\right)$ is the PDF value for PDF $P$ for the parton coming from the proton, $x_{p}$ is the fraction of the proton momentum carried by that parton, $f_{\bar{p}}^{P}\left(x_{\bar{p}}, Q\right) x_{\bar{p}}$ are the 
same for the parton coming from the antiproton, and $Q$ is the factorization scale. $Q$ is a process-dependent quantity, which for QCD $2 \rightarrow 2$ processes is

$$
Q^{2}=\hat{p}_{T}^{2}+\frac{m_{1}^{2}+m_{2}^{2}+m_{3}^{2}+m_{4}^{2}}{2}
$$

where $m_{1}$ and $m_{2}$ are the masses of the incoming partons from the proton and antiproton, $m_{3}$ and $m_{4}$ are the masses of the outgoing particles (leptoquarks, squarks, or gluinos in the possible signals considered in this analysis), and $\hat{p}_{T}$ is the total initial transverse momentum of the incoming partons.

With the events re-weighted, a new value of $A_{\text {sig }}$ can be calculated for a new PDF. The acceptance calculated using the default CTEQ5L PDF is compared with the variations in acceptance when using forty different CTEQ6M PDF sets. The CTEQ6M PDFs come in pairs, denoted as CTEQ6M $x \pm$, where $x$ runs from 1 to 20 . The variations in acceptance between CTEQ5L and each CTEQ6M $x \pm$ are determined. Each variation in a negative direction is added in quadrature, as is each variation in a positive direction. The total uncertainty in $A_{s i g}$ from these variations is found by taking the larger of these two sums, and symmetrizing it.

The acceptance may also change if the MRST PDF sets are used. The variation in $A_{\text {sig }}$ between CTEQ5L and the MRST72 (an MRST PDF which uses the same $\alpha_{s}$ value of CTEQ5L) is calculated. If it is larger than the variation from the CTEQ6M eigenvectors, the MRST72/CTEQ5L variation is used instead. If the MRST72/CTEQ5L variation is smaller than the CTEQ6M variation, it is neglected.

Finally, the variation between MRST72 and MRST75, a PDF set which differs from MRST72 in choice of $\alpha_{s}$, is calculated. This variation is added in quadrature to the larger of the MRST72/CTEQ5L or CTEQ6M/CTEQ5L variations to form the final contribution to $\Delta A_{\text {sig }}$ due to PDF choice. 


\subsubsection{Jet Energy Scale}

The uncertainty on $A_{s i g}$ due to the uncertainty on jet energy scale [27] is calculated by finding the relative change in $A_{\text {sig }}$ after rescaling all jets in the Monte Carlo signal sample by a factor $\pm 1 \sigma$ more or less than the standard scaling (discussed in Subsection 4.2.1). The resulting changes in the $E_{T}$ of the jets change the $H_{T}$ and $H_{T}$. If the new jet energy scale correction lowers the $E_{T}$ of one of the jets to below the $30 \mathrm{GeV}$ threshold, or raises a previously ignored third jet to above $30 \mathrm{GeV}$, it can also change the number of jets counted in the event. The uncertainty is symmetrized by taking the larger of the two relative uncertainties.

\subsubsection{Initial and Final State Radiation}

The emission of a gluon from either the partons in the initial state or from the quarks or gluons produced in the final state may increase the number of jets counted, and thus cause a signal event to be rejected by the dijet selection cuts. Uncertainty in the accuracy of the modeling of initial state radiation (ISR) and final state radiation (FSR) in the signal Monte Carlo is another source of $\Delta A_{\text {sig }}$. The procedure used to evaluate the uncertainty is based on that used by the CDF Top Quark physics group [68].

To determine the acceptance uncertainty from changes in ISR and FSR for a given signal, two additional PYTHIA Monte Carlo samples are generated. One has less ISR and FSR, while another has more ISR and FSR. The ISR is modified by varying the PYTHIA parameters governing $\Lambda_{Q C D}$ (the momentum scale used to determine the value of the strong coupling constant $\alpha_{s}$ ) and the scale factor for the transverse momentum scale of ISR evolution. Similarly, FSR is increased or decreased by changing the PYTHIA parameters for the $Q^{2}$ scale of the hard scattering and for the $\Lambda_{Q C D}$ value used in running $\alpha_{s}$ for parton showers.

The $A_{s i g}$ for these two new samples is compared to that of the original signal sample 
with the default ISR and FSR. The combined systematic uncertainty on $A_{s i g}$ due to uncertainty on ISR and FSR is taken to be

$$
\Delta A_{s i g}(\mathrm{ISR} / \mathrm{FSR})=\frac{1}{2}\left(\frac{A_{s i g}(\mathrm{less})-A_{s i g}}{A_{s i g}}+\frac{A_{s i g}(\text { more })-A_{s i g}}{A_{s i g}}\right),
$$

with $A_{s i g}($ less $)$ and $A_{s i g}$ (more) being the acceptances with ISR and ISR decreased or increased, respectively.

The uncertainties from ISR and FSR can also be found individually. In this method, four additional Monte Carlo samples are generated: one with ISR increased, one with ISR decreased, one with FSR increased, and one with FSR decreased. The $\Delta A_{\text {sig }}$ contributions for ISR and FSR are calculated much as in Equation 8.3, with each being added in quadrature to the other contributions. For the leptoquark interpretation, this is the method used. However, as the difference in the final $\Delta A_{s i g}$ value between the two methods turns out to be small (see Subsection 8.3.2), the simpler method is used for the MSSM interpretation.

\subsubsection{Luminosity}

The uncertainty on the luminosity measurement is $6.0 \%$ [23], as previously mentioned in Subsection 2.2.6. A $6.0 \%$ uncertainty is therefore included in $\Delta A_{s i g}$ for all signal samples.

\subsubsection{Monte Carlo Statistics}

A statistical uncertainty on the $A_{s i g}$ must also be calculated due to the finite size of the signal samples. The formula is:

$$
\Delta A_{s i g}(\text { statistics })=\frac{\sqrt{N A_{s i g}\left(1-A_{s i g}\right)}}{N}
$$

where $N$ is the number of events in the sample. 


\subsubsection{Efficiency of Clean-up Cuts}

The final contribution to $\Delta A_{\text {sig }}$ considered is the possibility of differences in the efficiencies of the clean-up cuts between signal and Monte Carlo. (See Section 4.3 for details on these cuts.) To calculate this contribution, the change in total yield after removing each individual cut is examined for the signal Monte Carlo sample in question. This effect is also examined for the data sample for three of the cleanup cuts - the requirement that the event vertex have six or more associated tracks, the requirement that the event EMF be above 0.1 , and the requirement for tracks to be matched to the second jet. A scale factor and associated uncertainty is assigned for each of these selection cuts, with the uncertainty covering the possibilities that the events removed from the data are either entirely background or entirely signal.

For the event EMF cut and the cut on the number of tracks associated with the event vertex, the scale factor was taken to be 1.000 , with a relative uncertainty taken to be the percentage change in events in the data sample when the cut was removed, minus the percentage difference in the signal Monte Carlo yield without that cut.

The uncertainty on the scale factor from requiring tracks to be matched to the second jet is the percentage change in events in the data without that cut, minus the percentage difference in the signal Monte Carlo yield, divided by two. The scale factor is 1.000 minus the magnitude of that uncertainty.

Without the requirements that a primary vertex is reconstructed or that no isolated tracks appear, a large number of additional background events will appear in the data. In this analysis, the observed percentage of events removed in the Monte Carlo are assumed to be an upper limit on the percentage of signal events removed in the data. This assumption rests on the fact that events from real data are more likely to contain additional tracks from which vertices can be reconstructed and additional energy deposition which makes the tracks less isolated. Hence, the scale factor for the isolated track veto is taken by finding the percent difference in Monte Carlo signal yield without the veto, dividing 
that by two, and adding it to 1.000. The uncertainty is the same percent difference, divided by two.

In the signal Monte Carlo samples considered, the reconstructed primary vertex requirement was not seen to make any difference to the number of events passing either the low or high kinematic signal cuts. The scale factor for this cut is therefore taken to be $1.000 \pm 0.000$.

For the scale factor from the selection cut on the $z$-position of the vertex, the results from Reference [69] are used for all possible signals. This gives a scale factor of $0.996 \pm$ 0.001 .

The scale factors from the primary vertex reconstruction cut, the six-tracks requirement, the $\left|z_{v t x}\right|<60 \mathrm{~cm}$ cut, the event EMF cut, the isolated track veto, and the second jet track matching are all multiplied together to form the total scale factor for a given signal. The raw $A_{\text {sig }}$ from the Monte Carlo is multiplied by this scale factor, and the total uncertainty on the scale factor is included as part of the total $\Delta A_{\text {sig }}$.

\subsection{Leptoquark Interpretation}

To interpret this analysis in terms of cross-section and mass limits on scalar leptoquarks decaying to quarks + neutrinos (as discussed in Chapter 6), Monte Carlo samples of pairproduced second-generation leptoquarks are generated using PYTHIA. These samples are generated with a range of masses between 50 to $200 \mathrm{GeV} / c^{2}$. The acceptance, uncertainty on acceptance, and expected yield for the low and high kinematic region is calculated for each sample. First- and third-generation leptoquark samples are also examined for $150 \mathrm{GeV} / c^{2}$ and $180 \mathrm{GeV} / c^{2}$. Table 8.3 gives the acceptance for each leptoquark sample examined. 


\subsubsection{Expected Signal Yields}

Table 8.4 shows the number of events expected in both the low and high kinematic regions, based on the known integrated luminosity of the MET45 data sample, the efficiency of the MET45 trigger, the acceptance determined from Monte Carlo analysis, and the scale factor obtained from Monte Carlo. The scale factors used for the low and high kinematic

region, shown in Tables 8.5 and 8.6, were based on the results for $150 \mathrm{GeV} / c^{2}$ leptoquark pair production. The efficiency used for the MET45 trigger came from the convolution of the trigger efficiency plot from Figure 3.1 with the $E_{T}$ distribution for $150 \mathrm{GeV} / c^{2}$ leptoquark pair production. The trigger efficiency for the low kinematic region (that is, for an $80 \mathrm{GeV} \not_{T}$ cut) is $99.28 \%$, while the efficiency for the high kinematic region (where the $E_{T}$ cut is $100 \mathrm{GeV}$ ) is $99.36 \%$.

\subsubsection{Uncertainties and Event Limits}

Table 8.7 shows the contributions to $\Delta A_{\text {sig }}$ for pair production of $150 \mathrm{GeV} / c^{2}$ leptoquarks, in both kinematic regions. The ISR and FSR contributions to $\Delta A_{s i g}$ are calculated individually. Added in quadrature, the total contribution to $\Delta A_{s i g}$ from ISR and FSR is $8.5 \%$ and $7.0 \%$ in the low and high kinematic regions, respectively. When the simplified method outlined in Subsection 8.2.3 which calculates the combined contributions from two modified Monte Carlo samples rather than four is used, the total contributions are found to be $7.2 \%$ and $3.9 \%$ in the low and high kinematic regions, respectively. As shown in Table 8.7, the total uncertainty $\Delta A_{\text {sig }}$ changes by less than $1 \%$ with this method $(0.7 \%$ for the low kinematic region, and $0.8 \%$ for the high kinematic region). Since the event limit is not sensitive to changes that small, the ISR and FSR uncertainties are calculated individually in the leptoquark interpretation.

Table 8.8 shows the same calculations for $\Delta A_{s i g}$ in pair production of $180 \mathrm{GeV} / c^{2}$ leptoquarks. Since $\Delta A_{\text {sig }}$ calculated from the $180 \mathrm{GeV} / c^{2}$ leptoquarks is lower than that 
found for the $150 \mathrm{GeV} / c^{2}$ case, a conservative limit can be set using the $150 \mathrm{GeV} / c^{2}$ result for $\Delta A_{\text {sig }}$ over the entire mass range.

With an $\Delta A_{\text {sig }}$ of $13.3 \%$ in the low kinematic region and $20.7 \%$ in the high kinematic region, the a priori 95\% CL upper limits on the number of leptoquark pair events are 309 and 71 respectively. When combining this result with the $A_{s i g}$ for each $M_{L Q}$ as given in Table 8.3, it is determined that the low kinematic region is the more sensitive region of the two for $M_{L Q}=140 \mathrm{GeV} / c^{2}$ and below, while the high kinematic region gives more sensitive a priori limits for $M_{L Q}=150 \mathrm{GeV} / c^{2}$ and above. Comparing the appropriate kinematic region's a priori results to the event yields in Table 8.4 leads to the conclusion that leptoquark masses between 50 and $170 \mathrm{GeV} / c^{2}$ are excluded a priori for the second generation.

The observed 95\% CL upper limits on the number of leptoquark events are 359 and 51 events, based on the low and high kinematic region results respectively. These observa-

tional limits rule out second-generation leptoquark masses between 50 and $190 \mathrm{GeV} / c^{2}$. Linear extrapolation between the expected number of events for 190 and $200 \mathrm{GeV} / c^{2}$ gives a final $95 \% \mathrm{CL}$ lower limit of $190 \mathrm{GeV} / c^{2}$ on second-generation scalar leptoquark mass.

\subsubsection{Leptoquark Generations}

For the first-generation leptoquarks at $180 \mathrm{GeV} / \mathrm{c}^{2}$ (the closest mass to the secondgeneration limit for which first-generation leptoquarks were examined), the acceptance is $1.1 \%$ larger in the low kinematic region, and $5.9 \%$ larger in the high kinematic region. Since these discrepancies are small compared to the uncertainties on $A_{s i g}$, the acceptances for first generation leptoquarks in this analysis are treated as consistent with second generation leptoquark results. Thus, the same mass limits and cross-section limits will be set for the first generation leptoquarks.

For third-generation leptoquarks at $180 \mathrm{GeV} / c^{2}$, the acceptance discrepancy is larger 
$-5.6 \%$ lower in the low kinematic region, and $17.6 \%$ lower in the high kinematic region. The acceptance is lower for these cases because the $b$-jets produced by third generation leptoquark decay are more likely to produce secondary electrons or muons, making the isolated track veto more likely to reject the event. The differences are used to rescale $A_{\text {sig }}$ for third-generation leptoquarks, changing the $95 \%$ CL limits by making the lower mass limit smaller and the upper cross-section limit higher. The 95\% CL lower mass limit is $178 \mathrm{GeV} / c^{2}$ for third-generation leptoquarks.

\subsubsection{Cross-section and Mass Limits}

The lower limits on leptoquark mass and the upper limits on leptoquark pair production are given in Table 8.9 for the three generations. Figure 8.1 shows the expected and observed $95 \%$ upper limits on the pair production cross-section of first- and secondgeneration scalar leptoquarks. The theoretical cross-sections from Reference [50] at $\mu=$ $2 M_{L Q}, M_{L Q}, M_{L Q} / 2$ are also graphed: the $95 \%$ lower limits on mass quoted in this analysis assume $\mu=2 M_{L Q}$, which gives the most conservative value. This figure also shows a discontinuity in the limit at $140 \mathrm{GeV} / c^{2}$, since the low kinematic region is a priori more sensitive at $M_{L Q} \leq 140 \mathrm{GeV} / c^{2}$, but the high kinematic region is more sensitive at higher masses.

\subsubsection{Kinematic Comparisons}

This analysis is a counting experiment, which is primarily sensitive to the number of events passing a set of cuts rather than their kinematic distributions. Nevertheless, it is interesting to examine the $E_{T}$ and $H_{T}$ distributions for some leptoquark examples, compared to the total background prediction and the data. Figure 8.2 compares the $H_{T}$ distribution found in the data with that for the total SM background prediction, and with the $H_{T}$ distribution for pair production of $180 \mathrm{GeV} / c^{2}$ second-generation leptoquarks stacked on top of the background prediction. As with Figure 5.31, the $H_{T}$ distri- 


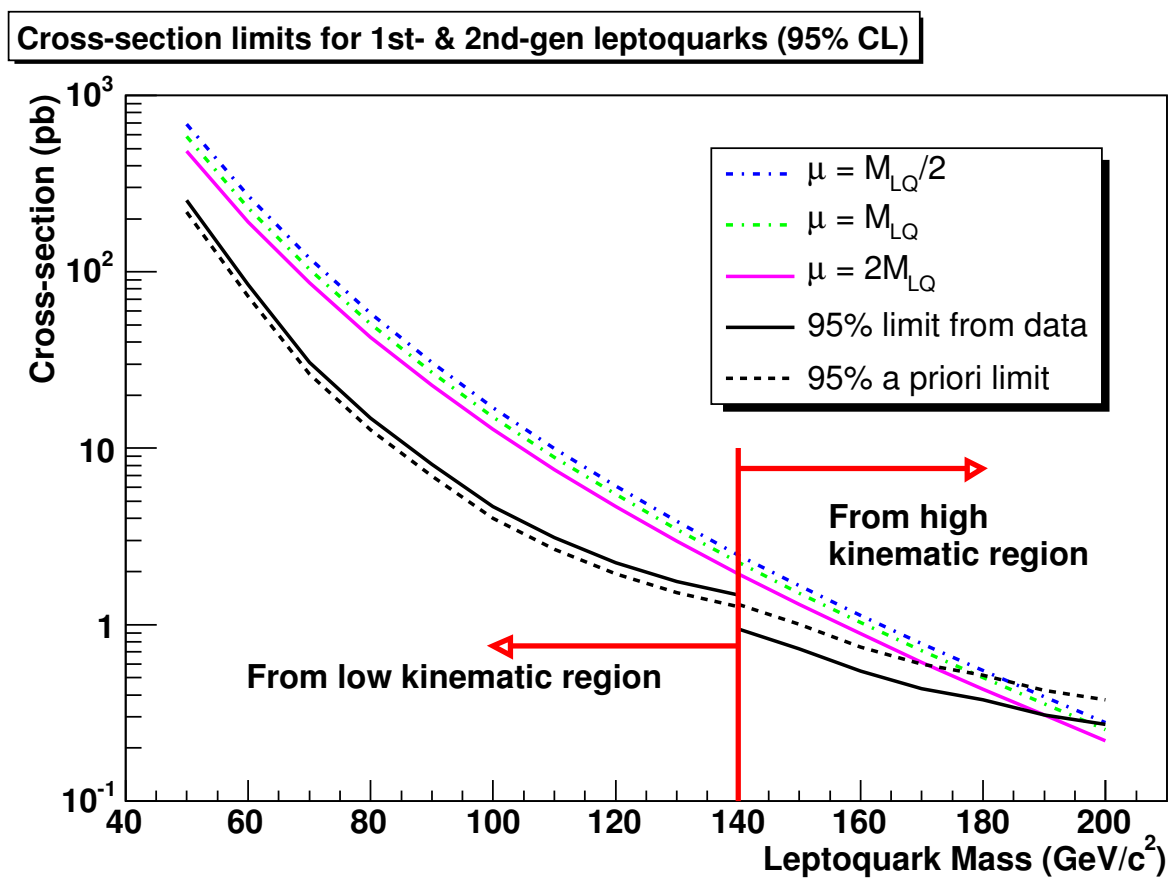

Figure 8.1: $95 \%$ cross-section limits for 1 st and 2nd generation leptoquark pair production as a function of $M_{L Q}$. Comparisons to theoretical cross-sections at $\mu=2 M_{L Q}, M_{L Q}, M_{L Q} / 2$ are also included.

bution associated with each SM background is normalized according to its contribution in the high kinematic region. Figures 8.3 and 8.4 show the $H_{T}$ and $F_{T}$ distributions, respectively, for data, the background prediction, and $180 \mathrm{GeV} / c^{2}$ second-generation leptoquarks in addition to predicted background for the high kinematic region. Since $180 \mathrm{GeV} / \mathrm{c}^{2}$ lies slightly below the limit on the leptoquark mass, this signal lies only slightly above the SM prediction and the observations in the data. 


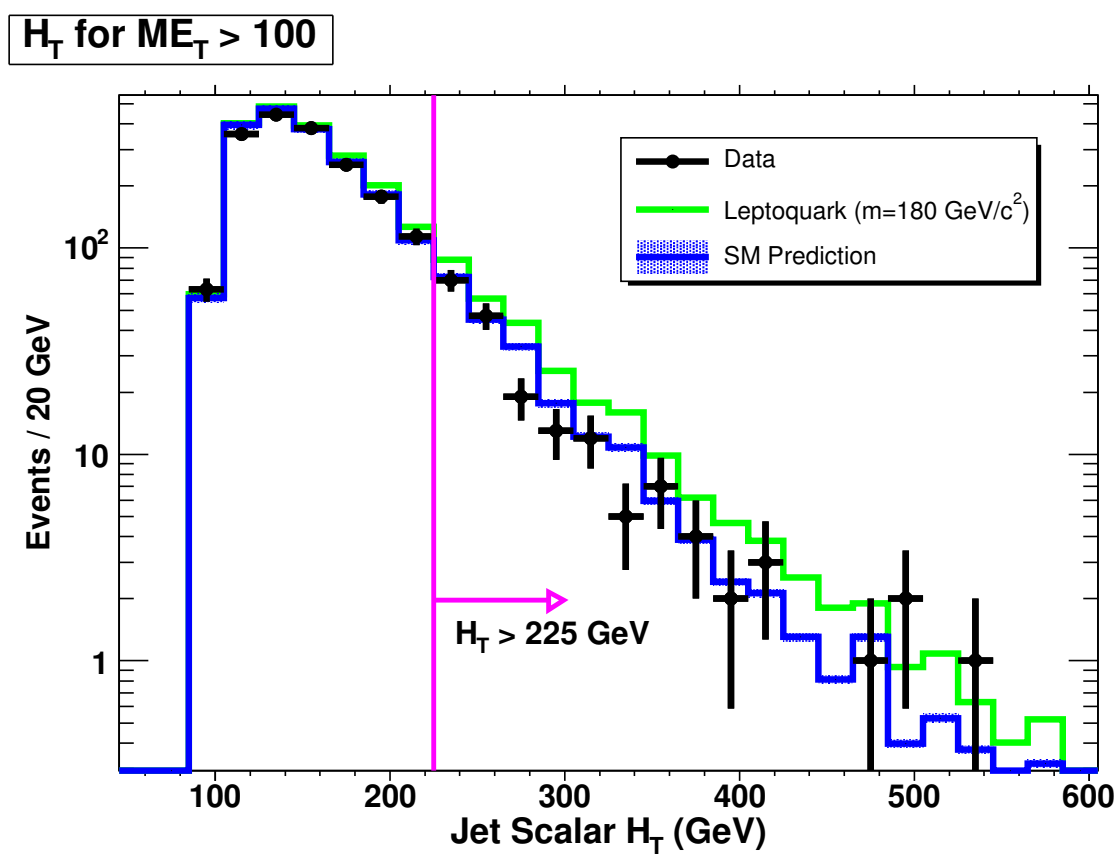

Figure 8.2: $H_{T}$ data, background prediction, and signal prediction after missing $E_{T}>100$ $\mathrm{GeV}$ cut. The signal is leptoquark pair production at $M_{L Q}=180 \mathrm{GeV} / c^{2}$, and is graphed assuming the $\sigma$ at $\mu=2 M_{L Q}$ of $0.432 \mathrm{pb}$. The region to the right of the vertical line at 225 $\mathrm{GeV}$ represents the high kinematic region. 


\begin{tabular}{|c|c|c|}
\hline$M_{L Q}\left(\mathrm{GeV} / c^{2}\right)$ & $H_{T}>125, \mathbb{E}_{T}>80$ & $H_{T}>225, E_{T}>100$ \\
\hline 50 & 0.07 & 0.01 \\
\hline 60 & 0.21 & 0.02 \\
\hline 70 & 0.58 & 0.06 \\
\hline 80 & 1.2 & 0.13 \\
\hline 90 & 2.2 & 0.29 \\
\hline 100 & 3.8 & 0.48 \\
\hline 110 & 5.7 & 0.81 \\
\hline 120 & 7.9 & 1.3 \\
\hline 130 & 10.1 & 1.9 \\
\hline 140 & 12.1 & 2.7 \\
\hline 150 & 13.9 & 3.5 \\
\hline 160 & 15.7 & 4.7 \\
\hline 170 & 17.1 & 5.9 \\
\hline 180 & 17.9 & 6.8 \\
\hline 190 & 19.3 & 8.3 \\
\hline 200 & 20.0 & 9.4 \\
\hline 150 (1st gen) & 14.1 & 4.0 \\
\hline 180 (1st gen) & 18.1 & 7.2 \\
\hline 150 (3rd gen) & 12.3 & 2.8 \\
\hline 180 (3rd gen) & 16.9 & 5.6 \\
\hline
\end{tabular}

Table 8.3: $A_{\text {sig }}$ (in \%) from Monte Carlo in both the low and high kinematic regions defined for the dijet plus $\mathbb{E}_{T}$ analysis as a function of second-generation leptoquark mass. Representative first- and third-generation results are also included. 


\begin{tabular}{|c|c|c|}
\hline$M_{L Q}\left(\mathrm{GeV} / c^{2}\right)$ & $H_{T}>125, E_{T}>80$ & $H_{T}>225, E_{T}>100$ \\
\hline 50 & 686 & 78 \\
\hline 60 & 830 & 70 \\
\hline 70 & 1020 & 108 \\
\hline 80 & 1060 & 111 \\
\hline 90 & 1026 & 134 \\
\hline 100 & 980 & 124 \\
\hline 110 & 868 & 124 \\
\hline 120 & 749 & 124 \\
\hline 130 & 604 & 114 \\
\hline 140 & 473 & 104 \\
\hline 150 & 364 & 93 \\
\hline 160 & 281 & 84 \\
\hline 170 & 213 & 73 \\
\hline 180 & 156 & 60 \\
\hline 190 & 120 & 52 \\
\hline 200 & 89 & 42 \\
\hline
\end{tabular}

Table 8.4: Expected number of signal events in both the low and high kinematic regions defined for the dijet plus $E_{T}$ analysis as a function of second-generation leptoquark mass. 


\begin{tabular}{|l|c|c|c|}
\hline Event Selection Cut & Data N-1 & MC N-1 & Scale Factor \\
\hline \hline Reconstructed Primary Vertex & N/A & $+0.0 \%$ & $1.000 \pm 0.000$ \\
\hline 6+ Tracks used to form Vertex & $+0.2 \%$ & $+0.0 \%$ & $1.000 \pm 0.002$ \\
\hline$\left|Z_{v t x}\right|<60 \mathrm{~cm}$ & N/A & N/A & $0.996 \pm 0.001$ \\
\hline Event EM Fraction $>0.1$ & $+1.0 \%$ & $+0.0 \%$ & $1.000 \pm 0.010$ \\
\hline Isolated Track Veto & N/A & $+2.0 \%$ & $1.010 \pm 0.010$ \\
\hline Tracks matched to 2nd Jet $(|\eta|<1.0)$ & $+2.8 \%$ & $+1.6 \%$ & $0.994 \pm 0.006$ \\
\hline \hline Total & & & $1.000 \pm 0.016$ \\
\hline
\end{tabular}

Table 8.5: Scale factors and associated uncertainties used to correct the signal acceptance obtained from Monte Carlo for measured event selection cut efficiencies in data in the low kinematic region for leptoquarks. Note that the scale factor uncertainties for the isolated track veto and $6+$ tracks used to form vertex requirements are assumed to be correlated.

\begin{tabular}{|l|c|c|c|}
\hline Event Selection Cut & Data N-1 & MC N-1 & Scale Factor \\
\hline \hline Reconstructed Primary Vertex & N/A & $+0.0 \%$ & $1.000 \pm 0.000$ \\
\hline $6+$ Tracks used to form Vertex & $+0.0 \%$ & $+0.0 \%$ & $1.000 \pm 0.000$ \\
\hline$\left|Z_{v t x}\right|<60 \mathrm{~cm}$ & N/A & N/A & $0.996 \pm 0.001$ \\
\hline Event EM Fraction $>0.1$ & $+5.9 \%$ & $+0.0 \%$ & $1.000 \pm 0.059$ \\
\hline Isolated Track Veto & N/A & $+3.6 \%$ & $1.018 \pm 0.018$ \\
\hline Tracks matched to 2nd Jet $(|\eta|<1.0)$ & $+4.9 \%$ & $+2.1 \%$ & $0.986 \pm 0.014$ \\
\hline \hline Total & & & $1.000 \pm 0.063$ \\
\hline
\end{tabular}

Table 8.6: Scale factors and associated uncertainties used to correct the signal acceptance obtained from Monte Carlo for measured event selection cut efficiencies in data in the high kinematic region for leptoquarks. 


\begin{tabular}{|l|c|c|}
\hline Uncertainty Source & $\Delta A_{\text {sig }}\left(H_{T}>125, E_{T}>80\right)$ & $\Delta A_{\text {sig }}\left(H_{T}>225, E_{T}>100\right)$ \\
\hline \hline Jet Energy Scale & $3.6 \%$ & $6.0 \%$ \\
\hline FSR (individually) & $1.6 \%$ & $2.6 \%$ \\
\hline ISR (individually) & $8.3 \%$ & $6.5 \%$ \\
\hline PDFs & $7.3 \%$ & $16.2 \%$ \\
\hline MC Statistics & $1.2 \%$ & $2.4 \%$ \\
\hline Luminosity & $6.0 \%$ & $6.0 \%$ \\
\hline Clean-up Cuts & $1.6 \%$ & $6.3 \%$ \\
\hline \hline Total & $13.3 \%$ & $20.7 \%$ \\
\hline \hline FSR/ISR & $7.2 \%$ & $3.9 \%$ \\
\hline Alternate Total & $12.6 \%$ & $19.9 \%$ \\
\hline
\end{tabular}

Table 8.7: $\Delta A_{\text {sig }}$ for $150 \mathrm{GeV} / c^{2}$ leptoquarks obtained from the Monte Carlo for both kinematic regions defined for the dijet plus $\mathbb{E}_{T}$ candidate sample.

\begin{tabular}{|l|c|c|}
\hline Uncertainty Source & $\Delta A_{\text {sig }}\left(H_{T}>125, E_{T}>80\right)$ & $\Delta A_{\text {sig }}\left(H_{T}>225, E_{T}>100\right)$ \\
\hline \hline Jet Energy Scale & $5.3 \%$ & $4.8 \%$ \\
\hline ISR (individually) & $6.8 \%$ & $5.4 \%$ \\
\hline FSR (individually) & $1.6 \%$ & $1.2 \%$ \\
\hline PDFs & $5.2 \%$ & $11.5 \%$ \\
\hline MC Statistics & $1.0 \%$ & $1.7 \%$ \\
\hline Luminosity & $6.0 \%$ & $6.0 \%$ \\
\hline Clean-up Cuts & $1.5 \%$ & $6.4 \%$ \\
\hline \hline Total & $12.0 \%$ & $16.3 \%$ \\
\hline
\end{tabular}

Table 8.8: $\Delta A_{\text {sig }}$ for $180 \mathrm{GeV} / c^{2}$ leptoquarks obtained from the Monte Carlo for both kinematic regions defined for the dijet plus $\not_{T}$ candidate sample. 


\begin{tabular}{|l|c|c|}
\hline LQ Model & Lower Mass Limit $\left(\mathrm{GeV} / c^{2}\right)$ & Upper Cross Section Limit (pb) \\
\hline \hline 1st Generation & 190 & 0.307 \\
\hline 2nd Generation & 190 & 0.307 \\
\hline 3rd Generation & 178 & 0.468 \\
\hline
\end{tabular}

Table 8.9: 95\% CL lower limits on scalar leptoquark mass for the different generations along with the corresponding upper limits on the leptoquark production cross-sections.

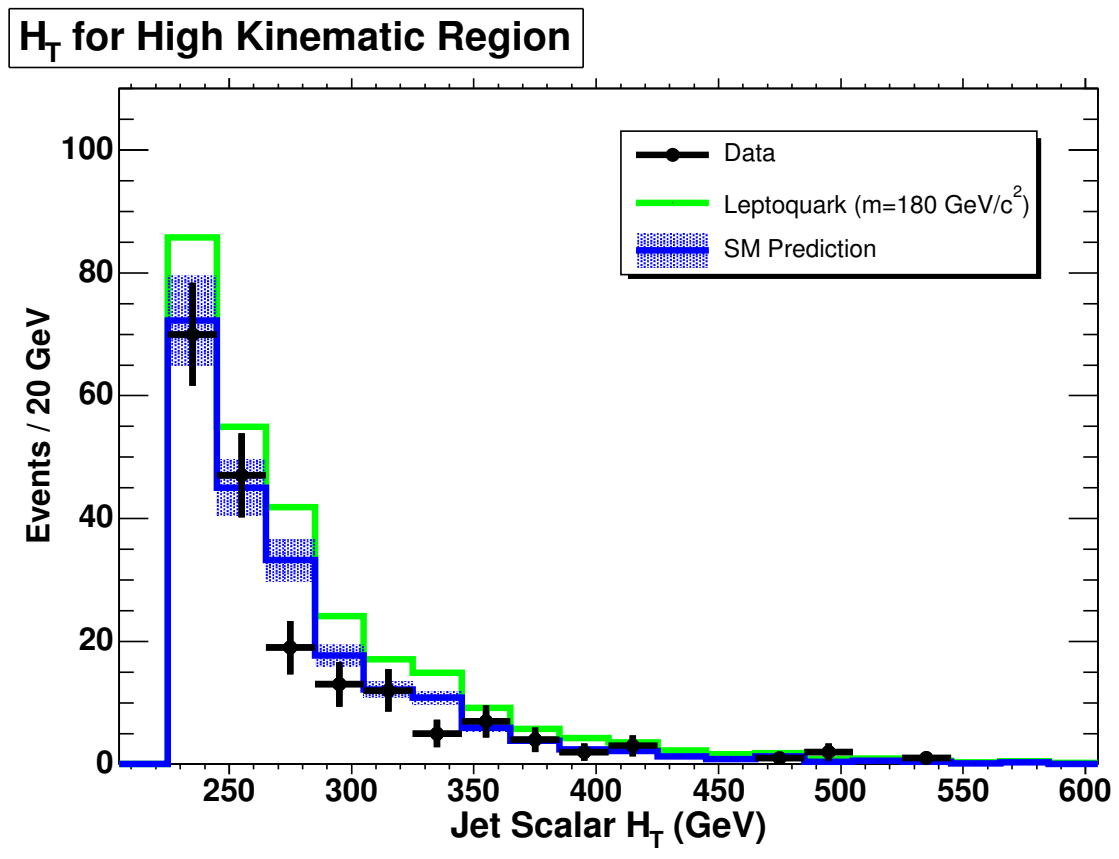

Figure 8.3: $H_{T}$ data, background prediction, and signal prediction for the high kinematic region. The signal is leptoquark pair production at $M_{L Q}=180 \mathrm{GeV} / c^{2}$, and is graphed assuming the $\sigma$ at $\mu=2 M_{L Q}$ of $0.432 \mathrm{pb}$. 


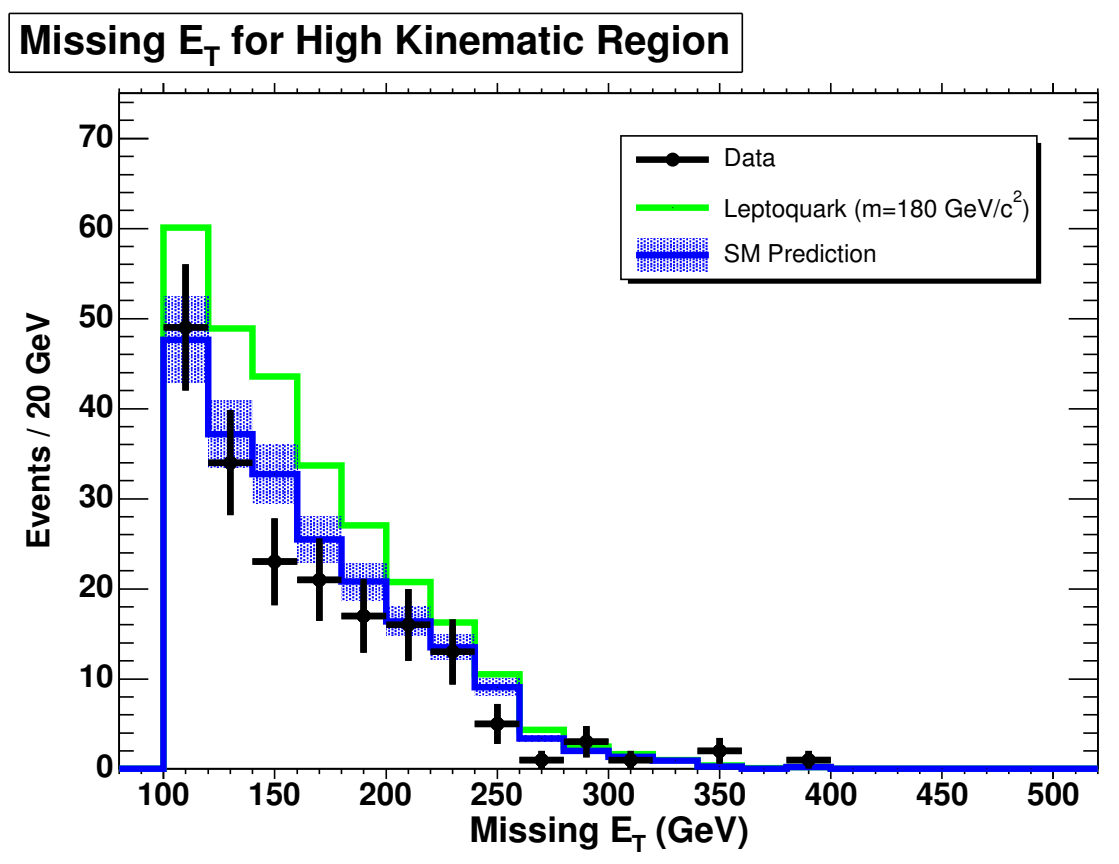

Figure 8.4: Missing $E_{T}$ data, background prediction, and signal prediction for the high kinematic region. The signal is leptoquark pair production at $M_{L Q}=180 \mathrm{GeV} / c^{2}$, and is graphed assuming the $\sigma$ at $\mu=2 M_{L Q}$ of $0.432 \mathrm{pb}$. 


\begin{tabular}{|c|c|c|c|}
\hline SUSY spectrum & LO $\sigma(\mathrm{pb})$ & $A_{\text {sig }}$ (low region) & $A_{\text {sig }}$ (high region) \\
\hline \multicolumn{4}{|c|}{ Right-handed $\tilde{q}$} \\
\hline \hline S1 & 0.045 & $28.0 \%$ & $21.9 \%$ \\
\hline S2 & 0.28 & $24.3 \%$ & $12.7 \%$ \\
\hline S3 & 0.61 & $20.2 \%$ & $6.8 \%$ \\
\hline S4 & 18.0 & $0.199 \%$ & $0.016 \%$ \\
\hline \multicolumn{4}{|c|}{ Inclusive } \\
\hline \hline S1 & 0.355 & $8.2 \%$ & $6.2 \%$ \\
\hline S2 & 1.7 & $8.5 \%$ & $3.7 \%$ \\
\hline S3 & 3.2 & $6.7 \%$ & $1.8 \%$ \\
\hline S4 & 57.3 & $0.222 \%$ & $0.018 \%$ \\
\hline
\end{tabular}

Table 8.10: PYThIA cross-sections and $A_{\text {sig }}$ (in \%) from Monte Carlo in both the low and high kinematic regions defined for the dijet $+\not_{T}$ analysis for the MSSM spectra examined.

\subsection{Supersymmetric Interpretation}

The results of this analysis can also be interpreted in terms of the non-mSUGRA MSSM model discussed in Chapter 7. Much as in the leptoquark case, cross-section limits are set on each mass spectrum examined by determining the acceptance for each spectrum using PYThia Monte Carlo samples. Cross-section limits are set for exclusive pair-production of right-handed squarks, as well as on the inclusive production of squark pairs, gluino pairs, and squark-gluino pairs combined. The cross-section limits found are compared to PYTHIA's leading order (LO) calculations. Table 8.10 gives the PYTHIA cross-sections and $A_{s i g}$ values for both kinematic regions for the four mass spectra studied. 


\begin{tabular}{|c|c|c|c|}
\hline SUSY spectrum & LO $\sigma(\mathrm{pb})$ & Yield (low region) & Yield (high region) \\
\hline \multicolumn{4}{|c|}{ Right-handed $\tilde{q}$} \\
\hline \hline S1 & 0.045 & 26 & 20 \\
\hline S2 & 0.28 & 139 & 72 \\
\hline S3 & 0.61 & 250 & 83 \\
\hline S4 & 18.0 & 73 & 6 \\
\hline \multicolumn{4}{|c|}{ Inclusive } \\
\hline \hline S1 & 0.355 & 59 & 44 \\
\hline S2 & 1.7 & 295 & 129 \\
\hline S3 & 3.2 & 433 & 116 \\
\hline S4 & 57.3 & 254 & 21 \\
\hline
\end{tabular}

Table 8.11: Leading order yields from PYTHIA for SUSY mass spectra examined.

\subsubsection{Expected Signal Yields}

Table 8.11 shows the number of events expected in both the low and high kinematic regions, based on the known integrated luminosity of the MET45 data sample, the efficiency of the MET45 trigger, the acceptance determined from Monte Carlo analysis, and the scale factor obtained from Monte Carlo. The results in this table assume the PYTHIA LO cross-sections.

The scale factors used for the low and high kinematic region are calculated separately for each spectrum. For both the inclusive and right-handed $\tilde{q}$ cases, the same scale factors are used. These scale factors are calculated primarily from the inclusive samples ${ }^{1}$. However, the isolated track scale factor is calculated using the right-handed $\tilde{q}$ samples.

\footnotetext{
${ }^{1}$ The scale factors for the inclusive and right-handed $\tilde{q}$ samples are very close: in the S2 sample, the inclusive scale factors come out to $1.000 \pm 0.049$ and $0.995 \pm 0.080$ in the high and low kinematic regions respectively, compared to $1.001 \pm 0.0049$ and $0.997 \pm 0.079$ in the right-handed $\tilde{q}$ samples. The same scale factors are thus used in order to simplify the analysis.
} 


\begin{tabular}{|l|c|c|c|}
\hline Event Selection Cut & Data N-1 & MC N-1 & Scale Factor \\
\hline \hline Reconstructed Primary Vertex & N/A & $+0.0 \%$ & $1.000 \pm 0.000$ \\
\hline 6+ Tracks used to form Vertex & $+0.2 \%$ & $+0.0 \%$ & $1.000 \pm 0.002$ \\
\hline$\left|Z_{v t x}\right|<60 \mathrm{~cm}$ & N/A & N/A & $0.996 \pm 0.001$ \\
\hline Event EM Fraction $>0.1$ & $+1.0 \%$ & $+0.0 \%$ & $1.000 \pm 0.010$ \\
\hline Isolated Track Veto & N/A & $+3.7 \%$ & $1.019 \pm 0.019$ \\
\hline Tracks matched to 2nd Jet $(|\eta|<1.0)$ & $+2.8 \%$ & $+0.9 \%$ & $0.990 \pm 0.010$ \\
\hline \hline Total & & & $1.005 \pm 0.050$ \\
\hline
\end{tabular}

Table 8.12: Scale factors and associated uncertainties used to correct the signal acceptance obtained from Monte Carlo for measured event selection cut efficiencies in data in the low kinematic region for MSSM spectrum S1.

This is because this contribution is meant to show the fraction of events lost to the isolated track veto in a sample which is not expected to have any leptons, and events with left-handed $\tilde{q}$ or $\tilde{g}$ decay may contain charged leptons from chargino decay, Tables 8.12 through 8.19 give the scale factors for the inclusive samples in the low and high kinematic regions.

The efficiency used for the MET45 trigger came from the convolution of the trigger efficiency plot from Figure 3.1 with the $\not_{T}$ distribution from the relevant S2 signal sample (either the right-handed $\tilde{q}$ sample or the inclusive sample.) The trigger efficiencies for the low kinematic region (that is, for an $80 \mathrm{GeV} \#_{T}$ cut) are $98.75 \%$ for the inclusive sample and $99.24 \%$ for the right-handed $\tilde{q}$ sample. For the high kinematic region (with a $100 \mathrm{GeV} \not_{T}$ cut) the efficiencies are $98.74 \%$ for the inclusive sample and $99.30 \%$ for the right-handed $\tilde{q}$ sample $^{2}$.

\footnotetext{
${ }^{2}$ In S3, the efficiencies for an $80 \mathrm{GeV}$ cut are $98.24 \%$ for the inclusive sample and $99.26 \%$ for the right-handed $\tilde{q}$ sample. For a $100 \mathrm{GeV}$ cut, the efficiencies are $99.30 \%$ for the inclusive sample and $99.31 \%$ for the right-handed $\tilde{q}$ sample. Since the trigger efficiency changes very little, S2's efficiencies are used for all four spectra.
} 


\begin{tabular}{|l|c|c|c|}
\hline Event Selection Cut & Data N-1 & MC N-1 & Scale Factor \\
\hline \hline Reconstructed Primary Vertex & N/A & $+0.0 \%$ & $1.000 \pm 0.000$ \\
\hline $6+$ Tracks used to form Vertex & $+0.0 \%$ & $+0.0 \%$ & $1.000 \pm 0.000$ \\
\hline$\left|Z_{v t x}\right|<60 \mathrm{~cm}$ & N/A & N/A & $0.996 \pm 0.001$ \\
\hline Event EM Fraction $>0.1$ & $+5.9 \%$ & $+0.0 \%$ & $1.000 \pm 0.059$ \\
\hline Isolated Track Veto & N/A & $+4.1 \%$ & $1.021 \pm 0.021$ \\
\hline Tracks matched to 2nd Jet $(|\eta|<1.0)$ & $+4.8 \%$ & $+1.0 \%$ & $0.981 \pm 0.019$ \\
\hline \hline Total & & & $0.997 \pm 0.079$ \\
\hline
\end{tabular}

Table 8.13: Scale factors and associated uncertainties used to correct the signal acceptance obtained from Monte Carlo for measured event selection cut efficiencies in data in the high kinematic region for MSSM spectrum S1.

\begin{tabular}{|l|c|c|c|}
\hline Event Selection Cut & Data N-1 & MC N-1 & Scale Factor \\
\hline \hline Reconstructed Primary Vertex & N/A & $+0.0 \%$ & $1.000 \pm 0.000$ \\
\hline $6+$ Tracks used to form Vertex & $+0.2 \%$ & $+0.1 \%$ & $1.000 \pm 0.001$ \\
\hline$\left|Z_{v t x}\right|<60 \mathrm{~cm}$ & N/A & N/A & $0.996 \pm 0.001$ \\
\hline Event EM Fraction $>0.1$ & $+1.0 \%$ & $+0.0 \%$ & $1.000 \pm 0.010$ \\
\hline Isolated Track Veto & N/A & $+2.9 \%$ & $1.015 \pm 0.015$ \\
\hline Tracks matched to 2nd Jet $(|\eta|<1.0)$ & $+2.8 \%$ & $+0.6 \%$ & $0.989 \pm 0.011$ \\
\hline \hline Total & & & $1.000 \pm 0.049$ \\
\hline
\end{tabular}

Table 8.14: Scale factors and associated uncertainties used to correct the signal acceptance obtained from Monte Carlo for measured event selection cut efficiencies in data in the low kinematic region for MSSM spectrum S2. 


\begin{tabular}{|l|c|c|c|}
\hline Event Selection Cut & Data N-1 & MC N-1 & Scale Factor \\
\hline \hline Reconstructed Primary Vertex & N/A & $+0.0 \%$ & $1.000 \pm 0.000$ \\
\hline $6+$ Tracks used to form Vertex & $+0.0 \%$ & $+0.0 \%$ & $1.000 \pm 0.000$ \\
\hline$\left|Z_{v t x}\right|<60 \mathrm{~cm}$ & N/A & N/A & $0.996 \pm 0.001$ \\
\hline Event EM Fraction $>0.1$ & $+5.9 \%$ & $+0.0 \%$ & $1.000 \pm 0.059$ \\
\hline Isolated Track Veto & N/A & $+4.1 \%$ & $1.020 \pm 0.020$ \\
\hline Tracks matched to 2nd Jet $(|\eta|<1.0)$ & $+4.8 \%$ & $+0.6 \%$ & $0.979 \pm 0.021$ \\
\hline \hline Total & & & $0.995 \pm 0.080$ \\
\hline
\end{tabular}

Table 8.15: Scale factors and associated uncertainties used to correct the signal acceptance obtained from Monte Carlo for measured event selection cut efficiencies in data in the high kinematic region for MSSM spectrum S2.

\begin{tabular}{|l|c|c|c|}
\hline Event Selection Cut & Data N-1 & MC N-1 & Scale Factor \\
\hline \hline Reconstructed Primary Vertex & N/A & $+0.0 \%$ & $1.000 \pm 0.000$ \\
\hline $6+$ Tracks used to form Vertex & $+0.2 \%$ & $+0.0 \%$ & $1.000 \pm 0.001$ \\
\hline$\left|Z_{v t x}\right|<60 \mathrm{~cm}$ & N/A & N/A & $0.996 \pm 0.001$ \\
\hline Event EM Fraction $>0.1$ & $+1.0 \%$ & $+0.0 \%$ & $1.000 \pm 0.010$ \\
\hline Isolated Track Veto & N/A & $+2.3 \%$ & $1.012 \pm 0.012$ \\
\hline Tracks matched to 2nd Jet $(|\eta|<1.0)$ & $+2.8 \%$ & $+0.8 \%$ & $0.990 \pm 0.010$ \\
\hline \hline Total & & & $0.998 \pm 0.048$ \\
\hline
\end{tabular}

Table 8.16: Scale factors and associated uncertainties used to correct the signal acceptance obtained from Monte Carlo for measured event selection cut efficiencies in data in the low kinematic region for MSSM spectrum S3. 


\begin{tabular}{|l|c|c|c|}
\hline Event Selection Cut & Data N-1 & MC N-1 & Scale Factor \\
\hline \hline Reconstructed Primary Vertex & N/A & $+0.0 \%$ & $1.000 \pm 0.000$ \\
\hline $6+$ Tracks used to form Vertex & $+0.0 \%$ & $+0.1 \%$ & $1.000 \pm 0.000$ \\
\hline$\left|Z_{v t x}\right|<60 \mathrm{~cm}$ & N/A & N/A & $0.996 \pm 0.001$ \\
\hline Event EM Fraction $>0.1$ & $+5.9 \%$ & $+0.0 \%$ & $1.000 \pm 0.059$ \\
\hline Isolated Track Veto & N/A & $+3.9 \%$ & $1.020 \pm 0.020$ \\
\hline Tracks matched to 2nd Jet $(|\eta|<1.0)$ & $+4.8 \%$ & $+1.3 \%$ & $0.981 \pm 0.019$ \\
\hline \hline Total & & & $0.996 \pm 0.079$ \\
\hline
\end{tabular}

Table 8.17: Scale factors and associated uncertainties used to correct the signal acceptance obtained from Monte Carlo for measured event selection cut efficiencies in data in the high kinematic region for MSSM spectrum S3.

\begin{tabular}{|l|c|c|c|}
\hline Event Selection Cut & Data N-1 & MC N-1 & Scale Factor \\
\hline \hline Reconstructed Primary Vertex & N/A & $+0.0 \%$ & $1.000 \pm 0.000$ \\
\hline $6+$ Tracks used to form Vertex & $+0.2 \%$ & $+0.0 \%$ & $1.000 \pm 0.002$ \\
\hline$\left|Z_{v t x}\right|<60 \mathrm{~cm}$ & N/A & N/A & $0.996 \pm 0.001$ \\
\hline Event EM Fraction $>0.1$ & $+1.0 \%$ & $+0.0 \%$ & $1.000 \pm 0.010$ \\
\hline Isolated Track Veto & N/A & $+1.0 \%$ & $1.005 \pm 0.005$ \\
\hline Tracks matched to 2nd Jet $(|\eta|<1.0)$ & $+2.8 \%$ & $+0.0 \%$ & $0.986 \pm 0.014$ \\
\hline \hline Total & & & $0.987 \pm 0.048$ \\
\hline
\end{tabular}

Table 8.18: Scale factors and associated uncertainties used to correct the signal acceptance obtained from Monte Carlo for measured event selection cut efficiencies in data in the low kinematic region for MSSM spectrum S4. 


\begin{tabular}{|l|c|c|c|}
\hline Event Selection Cut & Data N-1 & MC N-1 & Scale Factor \\
\hline \hline Reconstructed Primary Vertex & N/A & $+0.0 \%$ & $1.000 \pm 0.000$ \\
\hline 6+ Tracks used to form Vertex & $+0.0 \%$ & $+0.0 \%$ & $1.000 \pm 0.000$ \\
\hline$\left|Z_{v t x}\right|<60 \mathrm{~cm}$ & N/A & N/A & $0.996 \pm 0.001$ \\
\hline Event EM Fraction $>0.1$ & $+5.9 \%$ & $+0.0 \%$ & $1.000 \pm 0.059$ \\
\hline Isolated Track Veto & N/A & $+0.0 \%$ & $1.020 \pm 0.020$ \\
\hline Tracks matched to 2nd Jet $(|\eta|<1.0)$ & $+4.8 \%$ & $+0.0 \%$ & $0.976 \pm 0.024$ \\
\hline \hline Total & & & $0.972 \pm 0.078$ \\
\hline
\end{tabular}

Table 8.19: Scale factors and associated uncertainties used to correct the signal acceptance obtained from Monte Carlo for measured event selection cut efficiencies in data in the high kinematic region for MSSM spectrum S4.

\subsubsection{Uncertainties and Event Limits}

$\Delta A_{s i g}$ for the MSSM samples is calculated exactly as it is for the leptoquark case. The uncertainty is calculated separately for each of the four mass spectra, as shown in Tables 8.20 through 8.23 . The same uncertainties are used to set event limits for the inclusive and right-handed $\tilde{q}$ samples. Uncertainties for the S2 and S3 spectra are also calculated in the right-handed $\tilde{q}$ case. For $\mathrm{S} 2, \Delta A_{s i g}$ is $12.22 \%$ in the low kinematic region and $13.77 \%$ in the high kinematic region. For $\mathrm{S} 3, \Delta A_{\text {sig }}$ is $11.99 \%$ in the low kinematic region and $15.65 \%$ in the high kinematic region. Since the $\Delta A_{s i g}$ values in the inclusive samples are higher in both regions for both spectra, this analysis uses the $\Delta A_{s i g}$ from the inclusive samples in all cases to give a more conservative estimate.

Table 8.24 shows the $95 \%$ CL observed and a priori upper limits on the number of events on the number of MSSM signal events contributing to the low and high kinematic region candidate samples. When combined with the values for $A_{s i g}$ from Table 8.10 and the known integrated luminosity of the data sample, the a priori limits for the high 


\begin{tabular}{|l|c|c|}
\hline Uncertainty Source & $\Delta A_{\text {sig }}\left(H_{T}>125, E_{T}>80\right)$ & $\Delta A_{\text {sig }}\left(H_{T}>225, E_{T}>100\right)$ \\
\hline \hline Jet Energy Scale & $8.4 \%$ & $5.6 \%$ \\
\hline FSR/ISR & $8.0 \%$ & $7.8 \%$ \\
\hline PDFs & $3.7 \%$ & $3.9 \%$ \\
\hline MC Statistics & $1.5 \%$ & $1.8 \%$ \\
\hline Luminosity & $6.0 \%$ & $6.0 \%$ \\
\hline Clean-up Cuts & $5.0 \%$ & $8.0 \%$ \\
\hline \hline Total & $14.6 \%$ & $14.5 \%$ \\
\hline
\end{tabular}

Table 8.20: $\Delta A_{\text {sig }}$ for MSSM spectrum S1 obtained from the Monte Carlo for both kinematic regions defined for the dijet plus $\not_{T}$ candidate sample.

\begin{tabular}{|l|c|c|}
\hline Uncertainty Source & $\Delta A_{\text {sig }}\left(H_{T}>125, E_{T}>80\right)$ & $\Delta A_{\text {sig }}\left(H_{T}>225, E_{T}>100\right)$ \\
\hline \hline Jet Energy Scale & $7.8 \%$ & $2.6 \%$ \\
\hline FSR/ISR & $8.2 \%$ & $8.0 \%$ \\
\hline PDFs & $1.5 \%$ & $8.2 \%$ \\
\hline MC Statistics & $1.5 \%$ & $2.3 \%$ \\
\hline Luminosity & $6.0 \%$ & $6.0 \%$ \\
\hline Clean-up Cuts & $4.9 \%$ & $8.0 \%$ \\
\hline \hline Total & $13.9 \%$ & $15.6 \%$ \\
\hline
\end{tabular}

Table 8.21: $\Delta A_{\text {sig }}$ for MSSM spectrum S2 obtained from the Monte Carlo for both kinematic regions defined for the dijet plus $\not_{T}$ candidate sample. 


\begin{tabular}{|l|c|c|}
\hline Uncertainty Source & $\Delta A_{\text {sig }}\left(H_{T}>125, E_{T}>80\right)$ & $\Delta A_{\text {sig }}\left(H_{T}>225, E_{T}>100\right)$ \\
\hline \hline Jet Energy Scale & $4.3 \%$ & $5.8 \%$ \\
\hline FSR/ISR & $7.9 \%$ & $6.1 \%$ \\
\hline PDFs & $2.9 \%$ & $13.8 \%$ \\
\hline MC Statistics & $1.7 \%$ & $3.3 \%$ \\
\hline Luminosity & $6.0 \%$ & $6.0 \%$ \\
\hline Clean-up Cuts & $4.8 \%$ & $7.9 \%$ \\
\hline \hline Total & $12.3 \%$ & $19.2 \%$ \\
\hline
\end{tabular}

Table 8.22: $\Delta A_{\text {sig }}$ for MSSM spectrum S3 obtained from the Monte Carlo for both kinematic regions defined for the dijet plus $\not_{T}$ candidate sample.

\begin{tabular}{|l|c|c|}
\hline Uncertainty Source & $\Delta A_{\text {sig }}\left(H_{T}>125, E_{T}>80\right)$ & $\Delta A_{s i g}\left(H_{T}>225, E_{T}>100\right)$ \\
\hline \hline Jet Energy Scale & $8.2 \%$ & $44.4 \%$ \\
\hline FSR/ISR & $8.5 \%$ & $12.9 \%$ \\
\hline PDFs & $15.9 \%$ & $30.1 \%$ \\
\hline MC Statistics & $9.6 \%$ & $33.3 \%$ \\
\hline Luminosity & $6.0 \%$ & $6.0 \%$ \\
\hline Clean-up Cuts & $4.8 \%$ & $7.8 \%$ \\
\hline \hline Total & $23.4 \%$ & $65.2 \%$ \\
\hline
\end{tabular}

Table 8.23: $\Delta A_{\text {sig }}$ for MSSM spectrum S4 obtained from the Monte Carlo for both kinematic regions defined for the dijet plus $\not_{T}$ candidate sample. 


\begin{tabular}{|c|c|c|}
\hline SUSY spectrum & A priori & Observed \\
\hline \multicolumn{3}{|c|}{ Low kinematic region } \\
\hline S1 & 313 & 363 \\
\hline $\mathrm{S} 2$ & 311 & 361 \\
\hline S3 & 307 & 356 \\
\hline $\mathrm{S} 4$ & 347 & 406 \\
\hline \multicolumn{3}{|c|}{ High kinematic region } \\
\hline S1 & 66 & 49 \\
\hline S2 & 67 & 49 \\
\hline S3 & 70 & 51 \\
\hline $\mathrm{S} 4$ & 490 & 334 \\
\hline
\end{tabular}

Table 8.24: A priori and observed 95\% CL upper limits on the number of MSSM signal events contributing to the candidate samples for the four spectra examined. Note that these limits apply to both the right-handed $\tilde{q}$ pair production and inclusive squark/gluino cases.

kinematic region gives the best (i.e. lowest) cross-section limits for samples S1, S2, and S3. For S4, the low kinematic region give a better limit. Hence, the high kinematic region observations are used to calculate final cross-section limits for all spectra except for S4.

\subsubsection{Cross-section Limits}

The cross-section limits for the four MSSM spectra are given in Table 8.25. The ratio of the observed 95\% CL cross-section upper limits are also compared with the PYTHIA cross-sections. For spectra S2 and S3, the ratio is less than one in both the inclusive and the right-handed $\tilde{q}$ cases, meaning that these spectra can be taken to be ruled out at leading order. For S1 and S4, the ratio is greater than one in both cases. The ratio 


\begin{tabular}{|c|c|c|c|c|}
\hline SUSY spectrum & A priori (pb) & Observed (pb) & PYTHIA (pb) & Ratio \\
\hline \hline \multicolumn{5}{|c|}{ Right-handed $\tilde{q}$} \\
\hline \hline S1 & 0.15 & 0.11 & 0.045 & 2.41 \\
\hline S2 & 0.26 & 0.19 & 0.28 & 0.68 \\
\hline S3 & 0.51 & 0.37 & 0.61 & 0.61 \\
\hline S4 & 86.1 & 100.5 & 18.0 & 5.57 \\
\hline \multicolumn{5}{|c|}{ Inclusive } \\
\hline \hline S1 & 0.53 & 0.39 & 0.36 & 1.09 \\
\hline S2 & 0.90 & 0.65 & 1.73 & 0.38 \\
\hline S3 & 1.93 & 1.40 & 3.21 & 0.44 \\
\hline S4 & 78.5 & 91.6 & 57.4 & 1.60 \\
\hline
\end{tabular}

Table 8.25: Cross-section 95\% CL a priori and observed upper limits for all four SUSY spectra, compared to PYTHIA leading order cross-sections. The final column is the observed 95\% CL upper limit divided by the PYTHIA cross-sections.

can then be interpreted as a "k-factor" which would have to be applied to the PYTHIA cross-sections if these spectra were to be ruled out by these results.

\subsubsection{Kinematic Comparisons}

Although this analysis is a counting experiment which does not attempt to analyze the goodness of fit for any kinematic distributions, the $F_{T}$ and $H_{T}$ distributions can be graphed against the total background prediction and the data. Figure 8.5 compares the $H_{T}$ distribution found in the data with that for the total SM background prediction, and with the $H_{T}$ distribution for the S2 inclusive MSSM Monte Carlo. The $H_{T}$ distribution associated with each SM background is normalized according to its contribution in the high kinematic region. Figures 8.6 and 8.7 show the $H_{T}$ and $E_{T}$ distributions, 


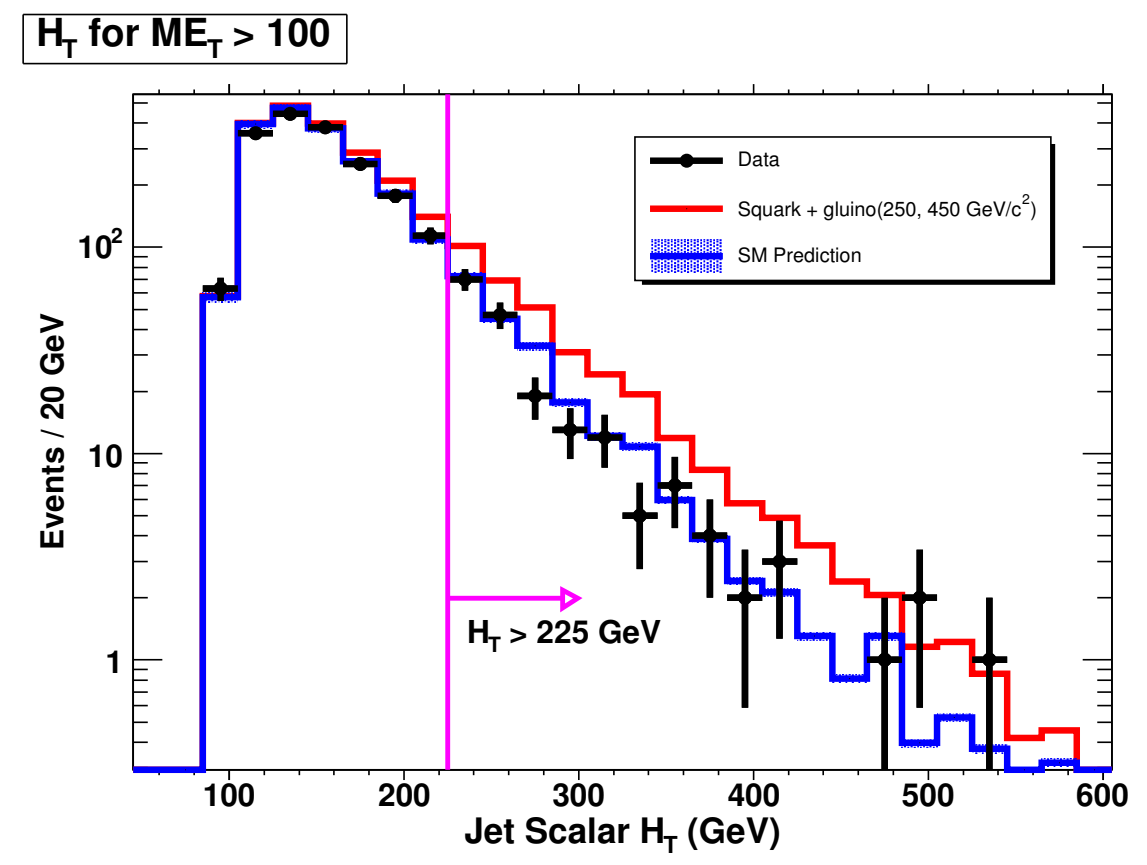

Figure 8.5: $H_{T}$ data, background prediction, and signal prediction after missing $E_{T}>100 \mathrm{GeV}$ cut. The signal is SUSY spectrum S2, and is graphed assuming the PYTHIA inclusive $\sigma$ of 1.73 $\mathrm{pb}$. The region to the right of the vertical line at $225 \mathrm{GeV}$ represents the high kinematic region. respectively, for data, the background prediction, and the S2 inclusive MSSM in addition to predicted background for the high kinematic region. In all three figures, the MSSM signal is graphed assuming the PYTHIA inclusive LO $\sigma$ of $1.73 \mathrm{pb}$. The MSSM signal is well in excess of the data, in agreement with the result from the counting experiment which sets the $95 \%$ CL cross-section upper limit to be $38 \%$ of the PYTHIA value. 


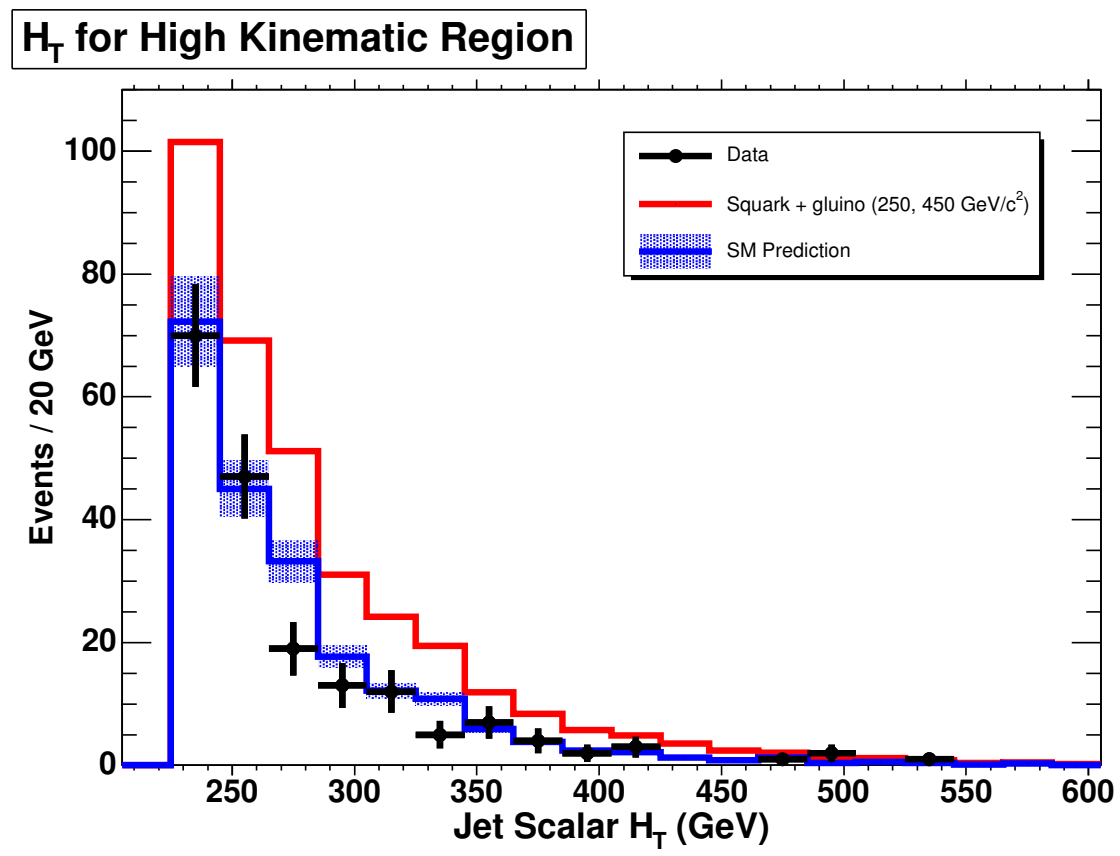

Figure 8.6: $H_{T}$ data, background prediction, and signal prediction for the high kinematic region. The signal is SUSY spectrum S2, and is graphed assuming the PYTHIA inclusive $\sigma$ of $1.73 \mathrm{pb}$.

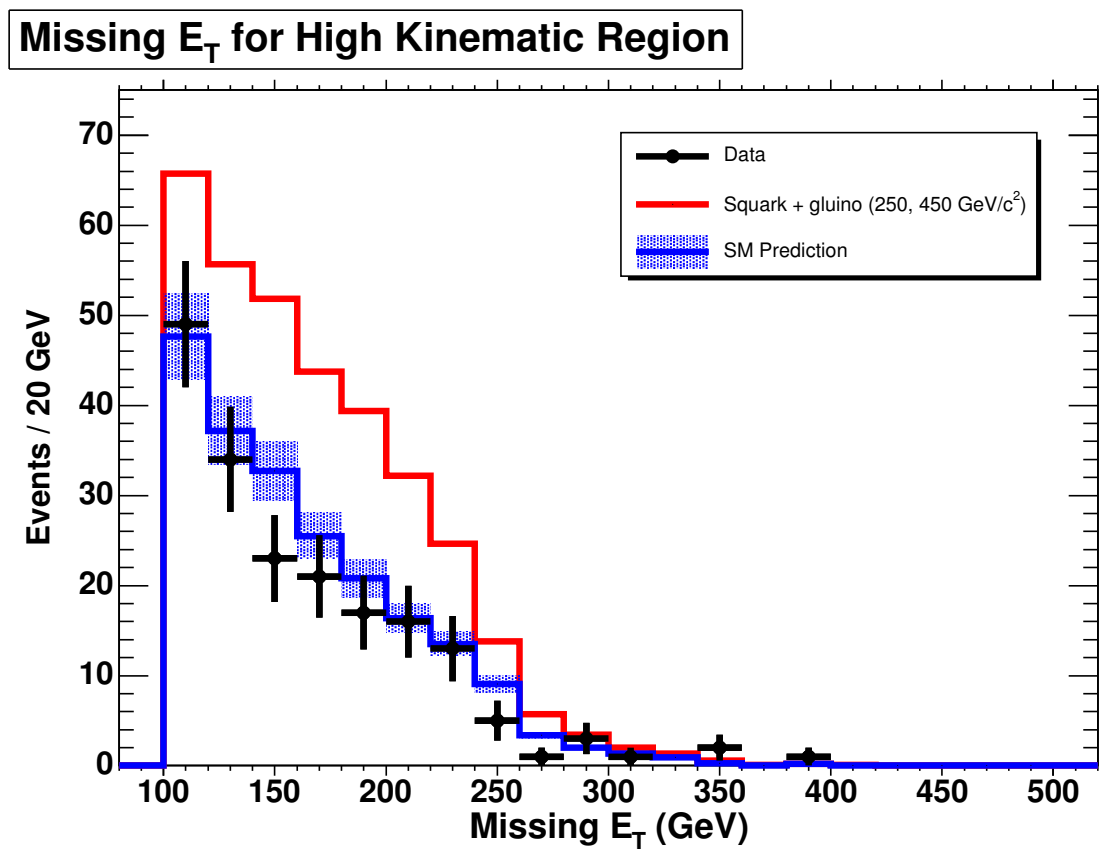

Figure 8.7: Missing $E_{T}$ data, background prediction, and signal prediction for the high kinematic region. The signal is SUSY spectrum S2, and is graphed assuming the PYTHIA inclusive $\sigma$ of $1.73 \mathrm{pb}$. 


\section{Chapter 9}

\section{Conclusion}

\subsection{Summary of Results}

This analysis searches for new physics in $p \bar{p}$ collisions, using $2 \mathrm{fb}^{-1}$ of data collected by the CDF-II detector. A signature-based search is used, allowing the results to be interpreted in a variety of models. The signature chosen is exclusive dijet production plus $E_{T}$ with additional jets vetoed. As described in Chapter 4, various cuts are applied to the data sample to cut down on background from "fake" $E_{T}$ arising from jet mismeasurement and processes producing leptons in the final state.

Two kinematic regions are defined using $H_{T}$ and $E_{T}$ cuts. The cuts for the "low" region, motivated by the efficiency of the MET45 trigger, are $H_{T}>125 \mathrm{GeV}$ and $\not_{T}>$ $80 \mathrm{GeV}$. For the "high" kinematic region, cuts of $H_{T}>225 \mathrm{GeV}$ and $\not_{T}>100 \mathrm{GeV}$ are motivated by the need for the cuts to still allow estimation of the background with an acceptably small relative uncertainty.

Data-driven estimates of the SM backgrounds for both kinematic regions are discussed in Chapter 5. The expectations are $2443 \pm 145$ events in the low kinematic region, and $211 \pm 30$ events in the high kinematic region. These results are consistent with the number of events actually found in the data (2506 events in the low region and 186 in the high 
region.) This allows the results to set limits on new physics.

Two possible models of new physics to which this signature would be sensitive are described in Chapters 6 (pair production of scalar leptoquarks) and 7 (production of squarks and gluinos in the minimal supersymmetric model.) Chapter 8 gives the results of this analysis in terms of $95 \%$ C.L. limits on both models. In the leptoquark case, 95\% C.L. lower mass limits of $190 \mathrm{GeV} / c^{2}$ for the first and second generation, and $178 \mathrm{GeV} / c^{2}$ for the third generation are determined. The 95\% C.L. cross-section upper limits are $0.307 \mathrm{pb}$ for the first two generations, and $0.468 \mathrm{pb}$ for the third generation. These leptoquark results improve on the previous best CDF mass limits by $62 \%$ to $20 \%$ depending on generation, and improve the cross-section limits by approximately one order of magnitude. While the third generation results are outdone by the previous $\mathrm{D} \varnothing$ results, the first and second-generation results are currently the best limits available for scalar leptoquarks decaying exclusively to quarks and neutrinos.

A non-mSUGRA MSSM model is also examined. 95\% cross-section upper limits as low as $0.39 \mathrm{pb}$ for inclusive $\tilde{g}+\tilde{q}$ production and $0.11 \mathrm{pb}$ for $\tilde{q}_{R}$ pair production can be set. Comparing the results to the cross-sections from PYTHIA, these limits allow us to rule out spectra with $\left(M_{\tilde{q}}, M_{\tilde{g}}, M_{\tilde{\chi}_{1}^{0}}\right)$ of $(250,450,72) \mathrm{GeV} / c^{2}$ and $(220,520,85) \mathrm{GeV} / c^{2}$ at $95 \%$ C.L. to leading order.

\subsection{Discussion}

In this analysis, model-independent limits are set on the production of events with a dijet $+E_{T}$ signature. These limits are applied to two different models of new physics: leptoquark production and a general supersymmetric model. Both models postulate pair

production of new particles (a scalar leptoquark or a right-handed squark, respectively) which each decay to produce a quark and a neutral particle which escapes undetected. Their primary difference comes from the nature of the invisible particle: in the leptoquark 
case, a massless neutrino is produced, while the neutralino produced from squark decay is massive.

The 95\% CL upper mass limit set on second-generation scalar leptoquarks is $190 \mathrm{GeV} / c^{2}$. However, squark production in MSSM spectrum S4, with a squark mass of $120 \mathrm{GeV} / c^{2}$, is not ruled out to leading order in this analysis. This is due to a much smaller acceptance in the MSSM case: $A_{s i g}$ is $0.199 \%$ for right-handed squark pair production for spectrum S4 using the low kinematic region cuts, compared to $7.9 \%$ for pair production of $120 \mathrm{GeV} / c^{2}$ scalar leptoquarks. The acceptance in this case is lower because so much of the energy from the squark decay is taken up by the massive neutralino. Thus, the events are less likely to pass the cuts set on $H_{T}$.

However, MSSM spectra S2 and S3, with squark masses of 250 and $220 \mathrm{GeV} / c^{2}$ respectively, are ruled out to leading order. This is because the leading order PYTHIA cross-sections used are larger than those for leptoquarks. According to PYTHIA calculations, the leading order cross-sections for pair production of four degenerate flavours of right-handed squarks is $0.28 \mathrm{pb}$ for spectrum $\mathrm{S} 2$ and $0.61 \mathrm{pb}$ for spectrum $\mathrm{S} 3$. This is well above $0.220 \mathrm{pb}$, the cross-section for $200 \mathrm{GeV} / c^{2}$ second-generation leptoquarks (the most massive leptoquarks examined in this analysis) at $\mu=2 M_{L Q}$, but this cross-section assumes only one flavour of leptoquark.

\subsection{Possibilities for Future Research}

The results in this thesis have obvious extensions for additional research. The leptoquark interpretation could be extended for different values of $\beta_{e}$, in the same way the previous CDF results from Reference [51] were used in References [55] and [56]. As for the MSSM interpretation, only a small fraction of the non-mSUGRA parameter space is explored in this thesis. These results could easily be extended to set limits on other MSSM mass spectra. Models of new physics other than the ones discussed could give a dijet + 
$E_{T}$ signature. The application of these results to models with both massive and massless invisible particles producing $E_{T}$ shows that a variety of new models could be examined with this signature.

The Tevatron is still running, with the CDF II detector still collecting data at the time of writing. Over $4 \mathrm{fb}^{-1}$ has been written to tape at $\mathrm{CDF}$, with approximately $8 \mathrm{fb}^{-1}$ of data-taking planned before the Tevatron ceases operation. Redoing this analysis with a larger data sample would improve the current limits.

The methods used in this analysis could easily be used for slightly different signatures. For example, the simple dijet requirement could be changed to require one or both jets to be tagged as coming from $b$ quarks. Such an analysis would be sensitive to pair production of third-generation leptoquarks with $L Q \rightarrow \bar{b} \nu_{\tau}$, or to production of $H+Z$ with $H \rightarrow b \bar{b}$ and $Z \rightarrow \nu \bar{\nu}$.

The Large Hadron Collider (LHC) at CERN in Switzerland began operation in 2008, and is expected to begin taking physics data in 2009. At the LHC, proton-proton collisions will take place with $\sqrt{s}=14 \mathrm{TeV}$, compared to $1.96 \mathrm{TeV}$ at the Tevatron. The design luminosity is $10^{34} \mathrm{~cm}^{-2} \mathrm{~s}^{-1}$ [71], compared to the current Tevatron records in the $10^{32} \mathrm{~cm}^{-2} \mathrm{~s}^{-1}$ range. With higher energy and, eventually, much greater integrated luminosity, LHC results in jets $+\not_{T}$ signatures should greatly extend existing limits on leptoquark and MSSM models. 


\section{Bibliography}

[1] S. L. Glashow, Partial-Symmetries of Weak Interactions, Nucl. Phys. 22, 588 (1961);

S. Weinberg, A Model of Leptons, Phys. Rev. Lett. 19, 1264 (1967).

[2] W. Marciano and H. Pagels, Quantum chromodynamics, Phys. Rept. 36, 137 (1978).

[3] A. Salam and J. C. Ward, Electromagnetic and weak interactions, Phys. Lett. 13, 168 (1964);

S. L. Glashow, J. Iliopoulos, and L. Maiani, Weak Interactions with Lepton-Hadron Symmetry, Phys. Rev. D 2, 1285 (1970);

M. Kobayashi and T. Maskawa, CP-Violation in the Renormalizable Theory of Weak Interaction, Prog. Theor. Phys. 49, 652 (1972).

[4] P. W. Higgs, Broken Symmetries and the Masses of Gauge Bosons, Phys. Rev. Lett. 13, 508 (1964).

[5] C. Amsler et al. (Particle Data Group), Review of Particle Physics, Phys. Lett. B 667,1 (2008).

[6] K. Kodama et al. (DONUT Collaboration), Observation of tau neutrino interactions, Phys. Lett. B 504, 218 (2001).

[7] The ALEPH, DELPHI, L3, and OPAL Collaborations, and the LEP Working Group for Higgs Boson Searches, Search for the Standard Model Higgs boson at LEP, Phys. Let. B 565, 61 (2003). 
[8] The ALEPH, DELPHI, L3, OPAL, and SLD Collaborations, Precision Electroweak Measurements on the Z Resonance, Phys. Rept. 427, 257 (2006)

[9] The TEVNPH Working Group for the CDF and D $\varnothing$ Collaborations, Combined CDF and DØ Upper Limits on Standard Model Higgs-Boson Production with up to 2.4 $\mathrm{fb}^{-1}$ of data, hep-ex/0804.3423v1 (2008).

[10] Reference [5] lists the following papers as the current best measurements of neutrino mixing angles:

B. Aharmim et al. (The SNO Collaboration), Electron energy spectra, fluxes, and day-night asymmetries of $8 B$ solar neutrinos from measurements with $\mathrm{NaCl}$ dissolved in the heavy-water detector at the Sudbury Neutrino Observatory, Phys. Rev. C 72, 005502 (2005);

Y. Ashie et al. (The Super-Kamiokande Collaboration), Measurement of atmospheric neutrino oscillation parameters by Super-Kamiokande I, Phys. Rev. D. 71, 112005 (2005);

M. Apollonio et al. (The CHOOZ Experiment), Limits on neutrino oscillations from the CHOOZ experiment, Phys. Let. B 466, 415 (1999).

[11] M. E. Peskin and D. V. Schroeder, An Introduction to Quantum Field Theory, Perseus Books, Cambridge, Massachusetts, 1995.

[12] For reviews, see for example:

L.M. Krauss, The Standard Model, Dark Matter and Dark Energy: From the Sublime to the Ridiculous, in the proceedings of the XIV Canary Islands Winter School in Astrophysics, astro-ph/0406673 (2002);

K.A. Olive, TASI Lectures on Dark Matter, in summary of lectures given at the Theoretical Advanced Study Institute in Elementary Particle Physics at the University of Colorado at Boulder, astro-ph/0301505 (2002);

and Reference [5]. 
[13] S. P. Martin, A Supersymmetry Primer, hep-ph/9709356, version 4, June 2006. This is an updated version of a contribution to Perspectives on Supersymmetry, ed. G. L. Kane, World Scientific, Singapore, 1998.

[14] W. Buchmüller, R. Rückl, and D. Wyler, Leptoquarks in Lepton-Quark Collisions, Phys. Let. B 191, 442 (1987); Erratum: Leptoquarks in Lepton-Quark Collisions Phys. Let. B 448, 320E (1999).

[15] Fermilab Visual Media Services, Fermilab Neg. \#: 00-0635D.

[16] B. Worthel, Linac Rookie Book v2.1, http://www-bdnew.fnal.gov/operations/ rookie_books/LINAC_v2.pdf, July 13, 2006.

[17] B. Worthel, Booster Rookie Book v3.1, http://www-bdnew.fnal.gov/operations/ rookie_books/Booster_v3_1.pdf, June 26, 2006.

[18] T. Asher, Main Injector Rookie Book v1.0, http://www-bdnew.fnal.gov/ operations/rookie_books/Main_Injector_v1.pdf, September 29, 2006.

[19] J. Morgan, The Antiproton Source Rookie Book v1.1, http://www-bdnew.fnal. gov/operations/rookie_books/Pbar_v1_1.pdf, July 14, 2006.

[20] B. Worthel, The Tevatron Rookie Book v1.0, http://www-bdnew.fnal.gov/ operations/rookie_books/Tevatron_v1.pdf, September 28, 2006.

[21] Fermilab Beams Division, Tevatron Luminosity webpage, http://www.fnal.gov/ pub/now/tevlum.html.

[22] R. Blair et al. (CDF Collaboration), The CDF II Detector Technical Design Report, FERMILAB-PUB-96-390-E, November 1996.

[23] A. Abulencia et al. (CDF Collaboration), Measurement of inclusive $W$ and $Z$ cross sections in $p \bar{p}$ collisions at $\sqrt{s}=1.98$ TeV, J. Phys. G Nucl. Part. Phys. 34, 2457 (2007). 
[24] A. Sill, for the CDF Collaboration, CDF Run II silicon tracking projects, Nucl. Instrum. Methods A447, 1 (2000).

[25] C. S. Hill, for the CDF Collaboration, Operational experience and performance of the CDFII silicon detector, Nucl. Instrum. Methods A530, 1 (2004).

[26] D. Acosta et al., for the CDF Collaboration, A Time-of-Flight detector in CDF-II, Nucl. Instrum. Methods A518, 605 (2004).

[27] A. Bhatti et al., Determination of the jet energy scale at the Collider Detector at Fermilab, Nucl. Intrum. Methods A556, 375 (2006).

[28] S. Kuhlmann et al., for the CDF Collaboration, The CDF calorimeter upgrade for Run IIb, Nucl. Instrum. Methods A518, 39 (2004).

[29] M. Albrow et al. (CDF Trigger and Datasets Working Group), CDF Run-II Trigger Table and Datasets Plan, CDF Note 4718, December 14, 2001.

[30] U. Grundler, L. Lovas, and A. Taffard, High- $P_{T}$ muons recommended cuts and efficiencies for Winter 2007, CDF Note 8618, June 1, 2006.

[31] T. Sjöstrand et al., High-Energy-Physics Event Generation with PYTHIA 6.1, Comput. Phys. Commun. 135, 238 (2001).

[32] G. Corcella et al., HERWIG 6: an event generator for hadron emission reactions with interfering gluons (including supersymmetric processes), J. High Energy Phys. JHEP01(2001)010.

[33] R. Brun et al., Simulation Program for Particle Physics Experiments. User Guide and Reference Manual, CERN-DD-78-2-REV.

[34] A. Abulencia et al. (CDF Collaboration), Search for Large Extra Dimensions in the Production of Jets and Missing Transverse Energy in p $\bar{p}$ Collisions at $\sqrt{s}=1.96$ TeV, Phys. Rev. Lett. 97, 171802 (2006). 
[35] M. Albrow et al., Missing $E_{T}$ and Beam related backgrounds at CDF, CDF Note 5926, August 13, 2002.

[36] H. K. Gerberich, A. V. Kotwal and C. Hays. Cosmic Ray Tagging using COT Hit Timing, CDF Note 6089, August 29, 2002.

[37] D. Hare, E. Halkiadakis, and T. Spreitzer, Electron ID Efficiency and Scale Factor for Winter 2007 Analyses, CDF Note 8614, December 7, 2006.

[38] M. L. Mangano et al., ALPGEN, a generator for hard multiproton processes in hadronic collisions, J. High Energy Phys. JHEP07(2003)001.

[39] R. D. Erbacher, Monte Carlo Tools: Report from CDF, LoopFest V, Stanford Linear Accelerator Laboratory, June 19-21, 2006;

M. L. Mangano, $A L P G E N$, KEK International Workshop on Physics simulations for the LHC, Tsukuba, April 5-6, 2004.

[40] K. Burkett, E. James, P.-H. Beauchemin, P.-O. Deviveiros, and P. Savard, An Esti-

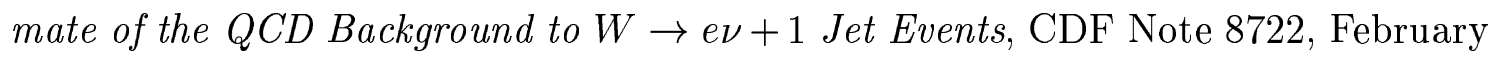
$28,2007$.

[41] J. Campbell and R.K. Ellis, Next-to-leading order corrections to $W+2$ jet and $Z+2$ jet production at hadron colliders, Phys. Rev. D 65, 113007 (2002).

[42] E. Thomson, R. Hughes, and C. Plager, Combination of CDF top quark pair production cross section measurements with up to $760 \mathrm{pb}^{-1}$, CDF Note 8148, April 10, 2006 .

[43] Z. Sullivan, Understanding single-top-quark production and jets at hadron colliders, Phys. Rev. D. 70, 114012 (2004);

[44] V. M. Abazov et al. (DØ Collaboration), Measurement of the Isolated Photon Cross Section in $p \bar{p}$ Collisions at $\sqrt{s}=1.96$ TeV, Phys. Lett. B 639, 151 (2006). 
[45] H. Georgi and S. Glashow, Unity of All Elementary-Particle Forces, Phys. Rev. Lett 32,438 (1974).

[46] J. C. Pati and A. Salam, Lepton number as the fourth "color", Phys. Rev. D 10, 275 (1974); Erratum: Lepton number as the fourth "color", Phys. Rev. D. 11, 703 (1975).

[47] J. L. Hewett and T. G. Rizzo, Much ado about leptoquarks: A comprehensive analysis, Phys. Rev. D. 56, 5709 (1997).

[48] M. Krämer, T. Plehn, M. Spira, and P. M. Zerwas, Pair Production of Scalar Leptoquarks at the Fermilab Tevatron, Phys. Rev. Lett. 79, 341 (1997).

[49] J. Blümlein, E. Boos, and A. Kryukov, Leptoquark Pair Production in Hadronic Interactions, DESY Report No. 96-174, hep-ph/9610408, October 1996.

[50] M. Krämer, private communication, 2008.

[51] D. Acosta et al. (CDF Collaboration), Search for scalar leptoquark pairs decaying to $\nu \bar{\nu} q \bar{q}$ in $p \bar{p}$ Collisions at $\sqrt{s}=1.96$ TeV, Phys. Rev. D 71, 112001 (2005).

[52] T. Affolder et al. (CDF Collaboration), Search for Second and Third Generation Leptoquarks Including Production via Technicolor Interactions in $p \bar{p}$ Collisions at $\sqrt{s}=1.8$ TeV, Phys. Rev. Lett. 85, 2056 (2000).

[53] V. M. Abazov et al. (DØ Collaboration), Search for Third-Generation Scalar Leptoquarks in $p \bar{p}$ collisions at $\sqrt{s}=1.96$ TeV, Phys. Rev. Lett. 99, 061801 (2007).

[54] V. M. Abazov et al. (DØ Collaboration), Search for scalar leptoquarks in the acoplanar jet topology in pp collisions at $\sqrt{s}=1.96$ TeV, Phys. Lett. B 640, 230 (2006).

[55] D. Acosta et. al (CDF Collaboration), Search for first-generation scalar leptoquarks in $p \bar{p}$ collisions at $\sqrt{s}=1.96$ TeV, Phys. Rev. D 72, 051107(R) (2005). 
[56] A. Abulencia et al. (CDF Collaboration), Search for second-generation scalar leptoquarks in $p \bar{p}$ collisions at $\sqrt{s}=1.96$ TeV, Phys. Rev. D 73, 051102(R) (2006).

[57] S. Chekanov et al. (ZEUS Collaboration), Search for resonance decays to lepton + jet at DESY HERA and limits on leptoquarks, Phys. Rev. D 68, 052004 (2003).

[58] L. Girardello and M. T. Grisaru, Soft breaking of supersymmetry, Nucl. Phys. B 194, 65 (1982).

[59] T. Affolder et al. (CDF Collaboration), Search for Gluinos and Scalar Quarks in $p \bar{p}$ Collisions at $\sqrt{s}=1.8 \mathrm{TeV}$ Using the Missing Energy plus Multijets Signature, Phys. Rev. Lett. 88, 041801 (2002).

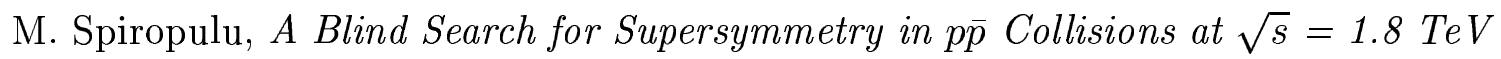
using the Missing Energy plus Multijet channel, Ph.D thesis, Harvard University, 2000.

[60] T. Aaltonen et al. (CDF Collaboration), Inclusive Search for Squark and Gluino Production in p $\bar{p}$ Collisions at $\sqrt{s}=1.96 \mathrm{TeV}$, submitted to Phys. Rev. Lett., arXiv:0811.2512;

Monica D'Onofrio, Gianluca De Lorenzo, Mario Martinez, Search for Gluino and Squark Production in Multijets Plus Missing $E_{T}$ Final States, CDF Note 9229 (Public Note.)

Older results for this analysis using a smaller data sample may be found in:

X. Portell, Searches for Squarks and Gluinos at CDF and DØ Detectors, Pub. Proceedings International Europhysics Conference on High Energy Physics (EPSHEP2007), Manchester, England, July 19-25, 2007. Fermilab-Conf-07-727-E (for $\left.1.1 \mathrm{fb}^{-1}\right)$;

X. Portell, Search for Gluino and Squark Production in Multi-jets plus Missing Transverse Energy Final States at the Tevatron using the CDF Detector, Ph.D. thesis, Universitat Autònoma de Barcelona, 2007 (for $371 \mathrm{pb}^{-1}$ ). 
[61] V. Abazov et al. (DØ collaboration), Search for squarks and gluinos in events with jets and missing transverse energy using $2.1 \mathrm{fb}^{-1}$ of $p \bar{p}$ collision data at $\sqrt{s}=1.96$ Te $V$, Phys. Lett. B660, 449 (2008).

Older results for this analysis using a smaller data sample may be found in:

V. Abazov et al. (DØ collaboration), Search for squarks and gluinos in events with jets and missing transverse energy using in p $\bar{p}$ collisions at $\sqrt{s}=1.96 \mathrm{Te} V$, Phys. Lett. B638, 119 (2006) (for $310 \mathrm{pb}^{-1}$ ).

[62] H. Baer, F. Paige, S. Protopescu, X. Tata, Isajet7.48: A Monte Carlo Event Generator for $p p, \bar{p} p$, and $e^{+} e^{-}$Interactions, hep-ph/0001086, Jan 11, 2000;

Simulating Supersymmetry with ISAJET 7.0/ISASUSY 1.0, hep-ph/9305342, May 28, 1993.

[63] LEPSUSYWG (LEP 2 Joint SUSY Working Group), ALEPH, DELPHI, L3 and OPAL experiments, Note LEPSUSYWG/02-06.2, http://lepsusy.web.cern.ch/ lepsusy/, November 25, 2002.

[64] J. Heinrich et al. (CDF Statistics Committee), Interval estimation in the presence of nuisance parameters. 1. Bayesian approach., CDF Note 7117 (Public Note), physics/0409129, September 27, 2004.

[65] H.L. Lai et al. (CTEQ Collaboration), Global QCD analysis of parton structure of the nucleon: CTEQ5 parton distributions, Eur.Phys.J.C 12, 375 (2000).

[66] J. Pumplin et al., New Generation of Parton Distributions with Uncertainties from Global QCD Analysis, JHEP 0207:012(2002), hep-ph/0201195;

D. Stump et al., Inclusive Jet Production, Parton Distributions, and the Search for New Physics, hep-ph/0303013.

[67] A.D. Martin et al.,MRST partons and uncertainties, hep-ph/0307262. 
[68] Y.-K. Kim and U.-K. Yang, Initial state gluon radiation studies on Drell-Yan data for top-pair production in hadron collider, CDF Note 6804, April 12, 2004.

[69] W. K. Sakumoto, Event $\left|Z_{v t x}\right|<60 \mathrm{~cm}$ Cut Acceptance for Run II, CDF Note 8318, June 12, 2006.

[70] CDF Joint Physics Group, PDF Uncertainties, http://www-cdf.fnal.gov/ internal/physics/joint_physics/instructions/PDFUncertainties/pdf . html, April 30, 2008;

Oscar González and Carsten Rott, Uncertainties due to the PDFs for the gluino-sbottom search, CDF Note 7051, June 2004.

[71] G. Aad et. al (ATLAS Collaboration), The ATLAS Experiment at the CERN Large Hadron Collider, 2008 JINST 3 S08003. 